\title{
Application of E1cB Elimination in Asymmetric Organocatalytic Cascade Reactions To Construct Polyheterocyclic Compounds
}

\author{
Zhi-Hao You, ${ }^{\dagger}$ Ying-Han Chen, ${ }^{\dagger}$ Yu Tang, ${ }^{\dagger,}$ and Yan-Kai Liu ${ }^{*},+$, \\ 'Key Laboratory of Marine Drugs, Chinese Ministry of Education, School of Medicine and Pharmacy, Ocean University of \\ China, Qingdao 266003, China. \\ "Laboratory for Marine Drugs and Bioproducts of Qingdao National Laboratory for Marine Science and Technology, \\ Qingdao 266003, China. \\ Email: liuyankai@ouc.edu.cn
}

Supporting Information 


\section{Table of contents}

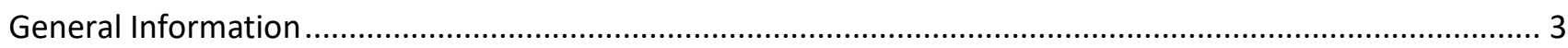

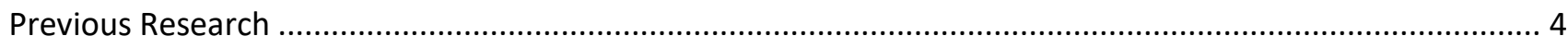

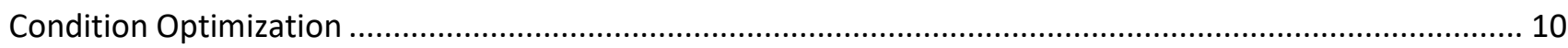

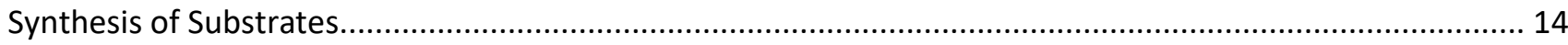

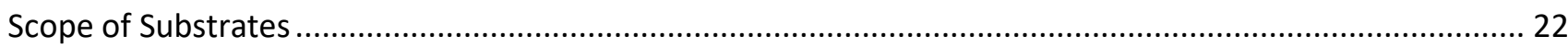

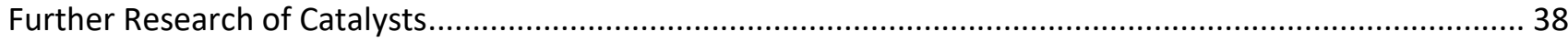

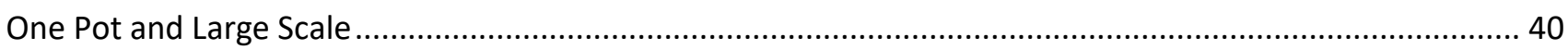

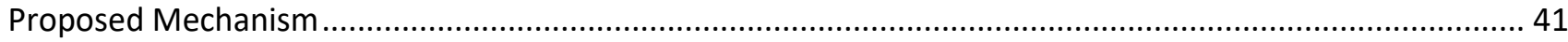

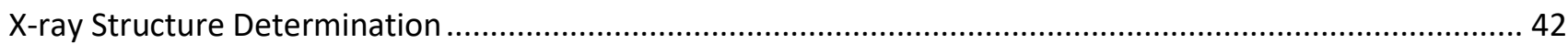

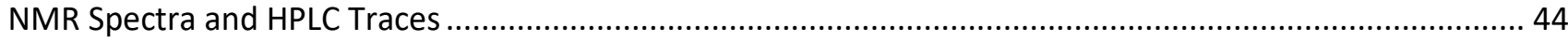




\section{General Information}

The ${ }^{1} \mathrm{H}$ and ${ }^{13} \mathrm{C}$ NMR spectra were recorded at $500 \mathrm{MHz}$ for ${ }^{1} \mathrm{H}$ and at $125 \mathrm{MHz}$ for ${ }^{13} \mathrm{C}$. The chemical shifts $(\delta)$ for ${ }^{1} \mathrm{H}$ and ${ }^{13} \mathrm{C}$ are given in ppm relative to residual signals of the solvents $\left(\mathrm{CDCl}_{3} @ 7.24\right.$ ppm ${ }^{1} \mathrm{H}$ NMR, 77.23 ppm ${ }^{13} \mathrm{C}$ NMR; d 6 DMSO @ 2.50 ppm ${ }^{1} \mathrm{H}$ NMR, 39.51 ppm ${ }^{13} \mathrm{C}$ NMR). Coupling constants are given in $\mathrm{Hz}$. The following abbreviations are used to indicate the multiplicity: s, singlet; $\mathrm{d}$, doublet; $\mathrm{t}$, triplet; $\mathrm{q}$, quartet; $\mathrm{m}$, multiplet. X-ray data were obtained from Zhongke chemical technology service center. High-resolution mass spectra (HRMS) were obtained from the Waters Q-Tof Ultima Global. Optical rotations are reported as follows: $[\alpha]_{\mathrm{D}}{ }^{20}$ (c in g per $100 \mathrm{~mL}, \mathrm{CHCl}_{3}$ as solvent). All the reactions were set up under air and using freshly distilled solvents, without any precautions to exclude moisture, unless otherwise noted. Chromatographic purification of products was accomplished using force-flow chromatography (FC) on silica gel (300-400 mesh). For thin layer chromatography (TLC) analysis throughout this work, Merck precoated TLC plates (silica gel 60 GF254, $0.25 \mathrm{~mm}$ ) were used, using UV light as the visualizing agent and an acidic mixture of ceric ammonium molybdate or basic aqueous potassium permanganate $\left(\mathrm{KMnO}_{4}\right)$ as stain developing solutions. Organic solutions were concentrated under reduced pressure on a Büchi rotary evaporator. HPLC analyses on chiral stationary phase were performed on Hitachi Chromaste. Daicel Chiralpak IA, IB or IC columns with $i-\mathrm{PrOH} / n$-hexane $/ \mathrm{CH}_{2} \mathrm{Cl}_{2}$ as the eluent were used. HPLC traces were compared to racemic samples obtained by using racemic catalysts. 


\section{Previous Research}

\section{Previous work of electrophiles Selection}

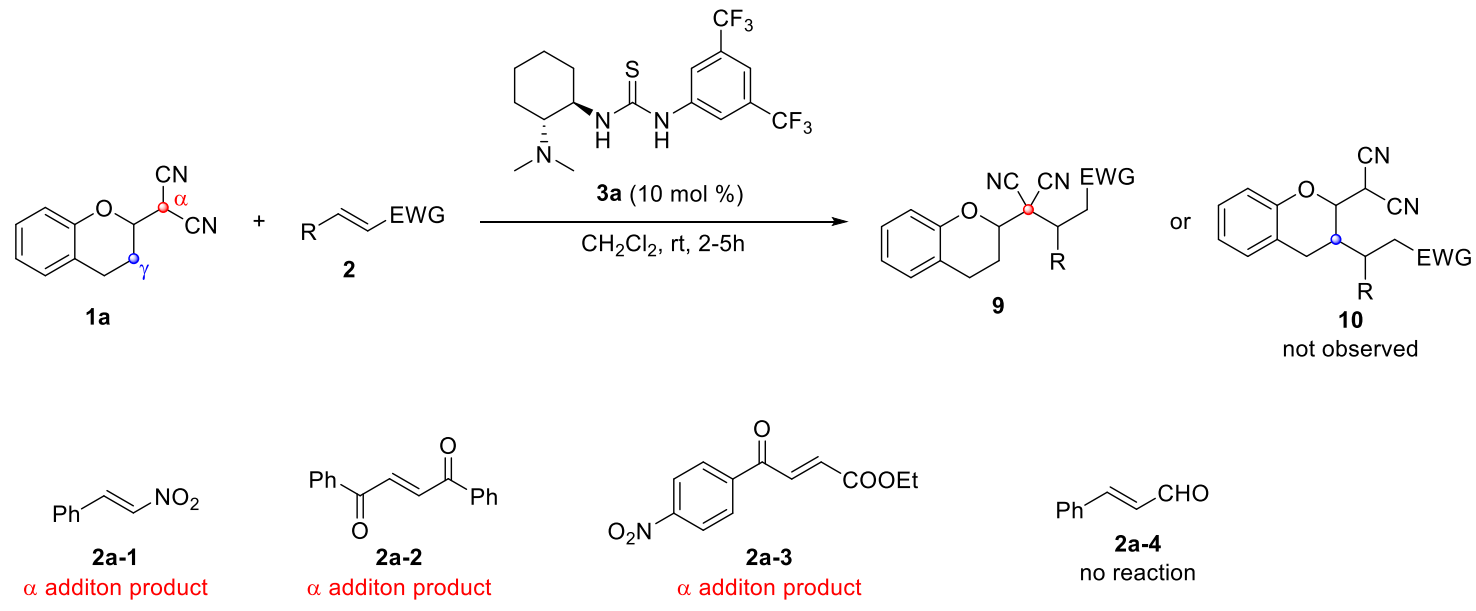

We first screened several Michael receptors as electrophiles.

All reactions were performed by the following procedure: compound $1 \mathbf{a}(19.8 \mathrm{mg}, 0.1 \mathrm{mmol})$ and $\mathbf{3 a}$ (10 mmol \%) were dissolved in $\mathrm{CH}_{2} \mathrm{Cl}_{2}(0.2 \mathrm{~mL})$ followed by addition of Michael receptor $2(0.11$ $\mathrm{mmol})$. Then the reaction was stirred at room temperature for 2 to 5 hours and purified by flash column chromatography on silica gel.

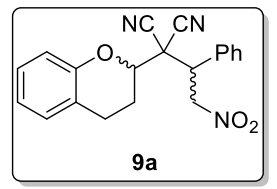

When 2a-1 was used as Michael receptor, only $\alpha$-addition product 9a $(\mathrm{dr}=1.4: 1)$ was obtained as white solid in $85 \%$ yield. ${ }^{1} \mathbf{H}$ NMR $\left(500 \mathrm{MHz}, \mathrm{CDCl}_{3}\right) \delta 7.49(\mathrm{dd}, J=$ 6.7, $2.8 \mathrm{~Hz}, 2 \mathrm{H}), 7.42-7.36(\mathrm{~m}, 3 \mathrm{H}), 7.21(\mathrm{t}, J=7.6 \mathrm{~Hz}, 1 \mathrm{H}), 7.05,(\mathrm{t}, J=8.7 \mathrm{~Hz}, 2 \mathrm{H})$, $6.99-6.93(\mathrm{~m}, 1 \mathrm{H}) .5 .22(\mathrm{~d}, J=7.9,2 \mathrm{H}), 4.71(\mathrm{t}, J=7.9 \mathrm{~Hz}, 1 \mathrm{H}), 3.73(\mathrm{dd}, J=11.5,2.1 \mathrm{~Hz}, 1 \mathrm{H}), 2.91-$ $2.74(\mathrm{~m}, 2 \mathrm{H}), 2.38-2.29(\mathrm{~m}, 1 \mathrm{H}), 2.25-2.14(\mathrm{~m}, 1 \mathrm{H}) \mathrm{ppm}$.

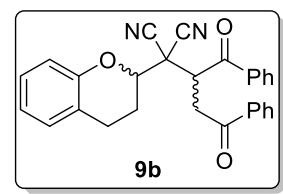

When $\mathbf{2 a - 2}$ was used as Michael receptor, only $\alpha$-addition product $\mathbf{9 b}(\mathrm{dr}=1.5: 1)$ was obtained as white solid in $64 \%$ yield. ${ }^{1} \mathrm{H}$ NMR $\left(500 \mathrm{MHz}, \mathrm{CDCl}_{3}\right) \delta 8.19-8.13$ (m, 2H), $7.99-7.91(m, 2 H), 7.60(t, J=7.4 \mathrm{~Hz}, 1 \mathrm{H}), 7.55,(t, J=7.4 \mathrm{~Hz}, 1 \mathrm{H}), 7.50-$ $7.41(\mathrm{~m}, 4 \mathrm{H}) .7 .05-6.93(\mathrm{~m}, 2 \mathrm{H}), 6.84(\mathrm{t}, J=7.4 \mathrm{~Hz}, 1 \mathrm{H}), 6.64(\mathrm{t}, J=8.1 \mathrm{~Hz}, 1 \mathrm{H}), 5.29$ (dd, $J=8.5,4.0$ $\mathrm{Hz}, 1 \mathrm{H}), 4.07$ (dd, $J=11.3,1.8 \mathrm{~Hz}, 1 \mathrm{H}), 3.97(\mathrm{dd}, J=18.0,8.5 \mathrm{~Hz}, 1 \mathrm{H}), 3.84(\mathrm{dd}, J=18.0,4.1 \mathrm{~Hz}, 1 \mathrm{H})$, $2.89-2.74(\mathrm{~m}, 2 \mathrm{H}), 2.52-2.44(\mathrm{~m}, 1 \mathrm{H}), 2.18(\mathrm{ddd}, J=24.7,11.6,6.5 \mathrm{~Hz}, 1 \mathrm{H}) \mathrm{ppm}$. 


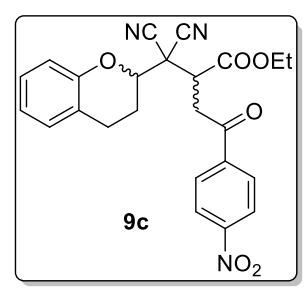

When 2a-3 was used as Michael receptor, only $\alpha$-addition product $9 \mathrm{c}(\mathrm{dr}=1.1: 1)$ was obtained as white solid in $85 \%$ yield. ${ }^{1} \mathrm{H}$ NMR $\left(500 \mathrm{MHz}, \mathrm{CDCl}_{3}\right) \delta 8.36(\mathrm{~d}, \mathrm{~J}=$ 8.7, $2 \mathrm{H}), 8.17(\mathrm{~d}, J=8.7,2 \mathrm{H}), 7.14-7.10(\mathrm{~m}, 2 \mathrm{H}), 6.93(\mathrm{t}, J=7.4 \mathrm{~Hz}, 1 \mathrm{H}), 6.83$, (d, $J=8.1 \mathrm{~Hz}, 1 \mathrm{H}), 4.52-4.42(\mathrm{~m}, 1 \mathrm{H}) .4 .26-4.10(\mathrm{~m}, 3 \mathrm{H}), 3.95(\mathrm{dd}, J=17.6,10.0$ $\mathrm{Hz}, 1 \mathrm{H}), 3.62(\mathrm{dd}, J=17.6,3.5 \mathrm{~Hz}, 1 \mathrm{H}), 3.04-2.84(\mathrm{~m}, 2 \mathrm{H}), 2.55-2.44(\mathrm{~m}, 1 \mathrm{H}), 2.22(\mathrm{dd}, J=11.6$, $11.5,6.6 \mathrm{~Hz}, 1 \mathrm{H}), 1.18(\mathrm{t}, J=7.1 \mathrm{~Hz}, 1 \mathrm{H}) \mathrm{ppm}$.

When $\mathbf{2 a - 4}$ was used as Michael receptor, there is no reaction observed under these conditions.

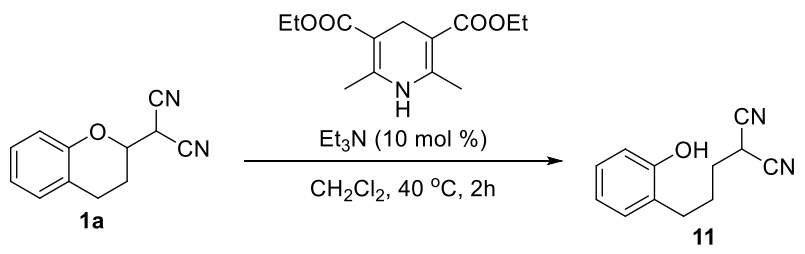

To our surprise, in the presence of $\mathrm{Et}_{3} \mathrm{~N}$ and Hantzsch ester, we were pleased to get the reduction product 11 from 1a, which indicated that the E1CB reaction governed ring-opening process could occur under basic conditions. The reaction was performed by the following procedure: compound 1a (19.8 mg, $0.1 \mathrm{mmol})$ and $\mathrm{Et}_{3} \mathrm{~N}(10 \mathrm{mmol} \%)$ were dissolved in $\mathrm{CH}_{2} \mathrm{Cl}_{2}(1 \mathrm{~mL})$ followed by addition of $\mathrm{HE}(30 \mathrm{mg}, 0.12 \mathrm{mmol})$. Then the reaction was stirred at $40{ }^{\circ} \mathrm{C}$ for $2 \mathrm{~h}$ and purified by flash column chromatography on silica gel. Product $\mathbf{1 1}$ was obtained as white solid in $77 \%$ yield. ${ }^{\mathbf{1}} \mathbf{H}$ NMR (500 $\left.\mathrm{MHz} \mathrm{CDCl}_{3}\right) \delta 7.13-7.05(\mathrm{~m}, 2 \mathrm{H}), 6.88(\mathrm{t}, J=7.2,1 \mathrm{H}), 6.71(\mathrm{~d}, J=7.8 \mathrm{~Hz}, 1 \mathrm{H}), 4.90(\mathrm{~s}, 1 \mathrm{H}), 3.80(\mathrm{t}, J$ $=7.0 \mathrm{~Hz}, 1 \mathrm{H}), 2.71(\mathrm{t}, J=7.0 \mathrm{~Hz}, 2 \mathrm{H}) .2 .09-2.00(\mathrm{~m}, 2 \mathrm{H}), 1.99-1.89(\mathrm{~m}, 2 \mathrm{H}) \mathrm{ppm}$.

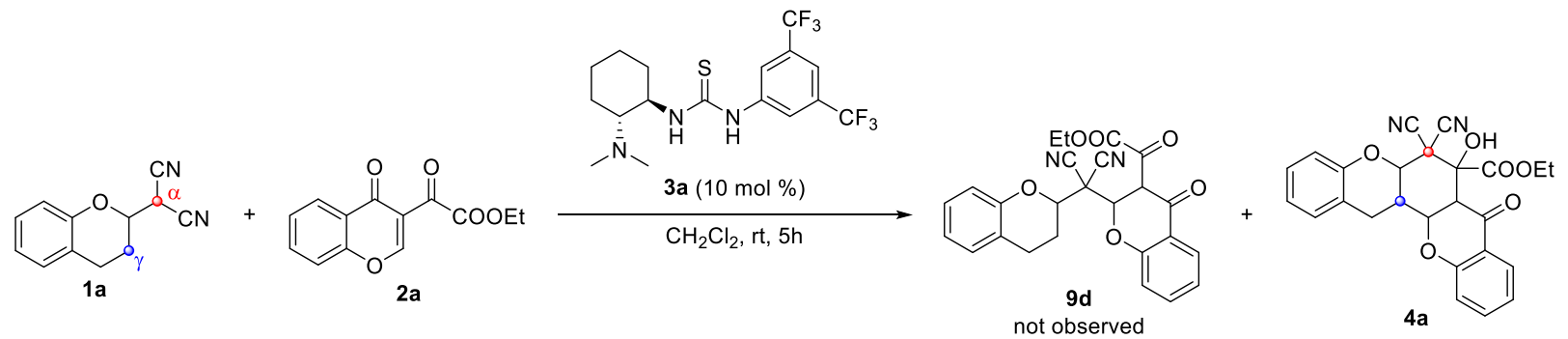

Encouraged by these results, we next moved toward the application of more reactive biselectrophilic substrate. To our surprise, when biselectrophilic chromone ketoester 2 a was used as electrophile in this reaction, no $\alpha$-addition product $9 \mathbf{d}$ was observed but only product $\mathbf{4 a}$ was obtained in $82 \%$ yield in the presence of Takemoto catalyst 3a. The reaction was performed by the 
following procedure: compound $1 \mathbf{a}(19.8 \mathrm{mg}, 0.1 \mathrm{mmol})$ and $\mathbf{3 a}(10 \mathrm{mmol} \%)$ were dissolved in $\mathrm{CH}_{2} \mathrm{Cl}_{2}(0.2 \mathrm{~mL})$ followed by addition of $2 \mathrm{a}(0.11 \mathrm{mmol})$. Then the reaction was stirred at room temperature for 5 hours and purified by flash column chromatography on silica gel.

Some other compounds, which could be used in this E1cB strategy, were synthesized and used to react with substrate $\mathbf{2 a}$. 


\section{Preparation of other E1CB substrates}

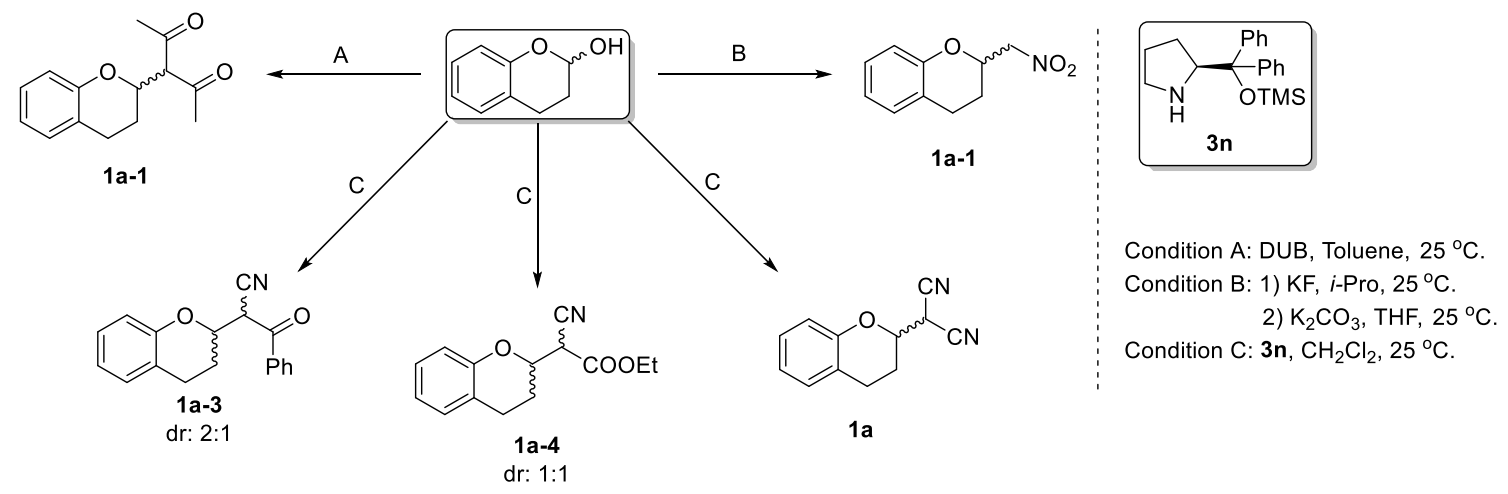

Condition A: Lactol $(75 \mathrm{mg}, 0.5 \mathrm{mmol})$ and acetylacetone $(50 \mathrm{mg}, 0.5 \mathrm{mmol})$ were dissolved in toluene $(1 \mathrm{~mL})$. DBU (20 mol \%) was added and the reaction was stirred at $25{ }^{\circ} \mathrm{C}$ until lactol was consumed completely. The reaction was purified by flash column chromatography on silica gel to give $1 \mathrm{a}-1$ in $50 \%$ yield. $1 \mathrm{a}-\mathbf{1}^{1} \mathbf{H}$ NMR $\left(500 \mathrm{MHz}, \mathrm{CDCl}_{3}\right) \delta 7.08-7.00(\mathrm{~m}, 2 \mathrm{H}), 6.86-6.82(\mathrm{~m}, 1 \mathrm{H})$, $6.72(\mathrm{~d}, J=8.1 \mathrm{~Hz}, 1 \mathrm{H}), 4.70(\mathrm{td}, J=9.8,2.1 \mathrm{~Hz}, 1 \mathrm{H}), 3.99(\mathrm{~d}, J=9.5 \mathrm{~Hz}, 1 \mathrm{H}), 2.93-2.84(\mathrm{~m}, 6.1 \mathrm{~Hz}$, $1 \mathrm{H}), 2.77-2.69(\mathrm{~m}, 1 \mathrm{H}), 2.30(\mathrm{~s}, 3 \mathrm{H}), 2.24(\mathrm{~s}, 3 \mathrm{H}), 2.09-2.01(\mathrm{~m}, 1 \mathrm{H}), 1.69-1.58(\mathrm{~m}, 1 \mathrm{H}) \mathrm{ppm} .{ }^{13} \mathrm{C}$ NMR $\left(125 \mathrm{MHz}, \mathrm{CDCl}_{3}\right) \delta 202.1,201.7,153.9,129.5,127.3,121.5,120.8,116.8,74.5,73.4,30.8$, $29.3,25.5,24.2 \mathrm{ppm}$.

Condition B: Lactol $(75 \mathrm{mg}, 0.5 \mathrm{mmol})$ and nitromethane (61 mg, $1 \mathrm{mmol}$ ) were dissolved in isopropyl alcohol ( $1 \mathrm{~mL})$. KF (20 mol \%) was added and the reaction was stirred at $\mathrm{rt}$ for 24 hour. Then the solvent was concentrated and dissolved in THF (1 mL). $\mathrm{K}_{2} \mathrm{CO}_{3}(276 \mathrm{mg}, 2 \mathrm{mmol})$ was added and the reaction was stirred at $25^{\circ} \mathrm{C}$ until completed. The reaction was filtered, concentrated and purified by flash column chromatography on silica gel to give $\mathbf{1 a - 2}$ in $36 \%$ yield. $\mathbf{1 a - 2}{ }^{\mathbf{1}} \mathbf{H}$ NMR (500 $\left.\mathrm{MHz}^{\mathrm{CDCl}}{ }_{3}\right) \delta 7.11(\mathrm{t}, J=7.7 \mathrm{~Hz}, 1 \mathrm{H}), 7.06(\mathrm{~d}, J=7.5 \mathrm{~Hz}, 1 \mathrm{H}), 6.89(\mathrm{t}, J=7.4 \mathrm{~Hz}, 1 \mathrm{H}), 6.81(\mathrm{~d}, J=8.1$ $\mathrm{Hz}, 1 \mathrm{H}), 4.82-4.74(\mathrm{~m}, 1 \mathrm{H}), 4.67(\mathrm{dd}, J=12.5,8.4 \mathrm{~Hz}, 1 \mathrm{H}), 4.54(\mathrm{dd}, J=12.5,3.8 \mathrm{~Hz}, 1 \mathrm{H}), 2.95$ (ddd, $J=16.9,11.0,6.1 \mathrm{~Hz}, 1 \mathrm{H}), 2.86-2.78(\mathrm{~m}, 1 \mathrm{H}), 2.14-2.07(\mathrm{~m}, 1 \mathrm{H}), 1.92-1.81(\mathrm{~m}, 1 \mathrm{H}) \mathrm{ppm} .{ }^{13} \mathrm{C}$ NMR $\left(125 \mathrm{MHz}, \mathrm{CDCl}_{3}\right) \delta 153.5,129.7,127.8,121.3,121.0,117.2,78.9,72.1,24.8,24.1$ ppm.

Condition C: Lactol $(75 \mathrm{mg}, 0.5 \mathrm{mmol})$ and correspondine cyanide $(0.5 \mathrm{mmol})$ were dissolved in $\mathrm{CH}_{2} \mathrm{Cl}_{2}(1 \mathrm{~mL}) .3 \mathrm{n}(5 \mathrm{~mol} \%)$ was added and the reaction was stirred at $25{ }^{\circ} \mathrm{C}$ until lactol was consumed completely. The reaction mixture was purified by flash column chromatography on silica gel to give corresponding products. 
1a-3 was obtained as a white solid in $46 \%$ yield $(\mathrm{dr}=2: 1) .{ }^{1} \mathbf{H} \mathrm{NMR}\left(500 \mathrm{MHz}, \mathrm{CDCl}_{3}\right) \delta 8.02(\mathrm{t}, J=8.6$ $\mathrm{Hz}, 2 \mathrm{H}), 7.71-7.63(\mathrm{~m}, 1 \mathrm{H}), 7.58-7.50(\mathrm{~m}, 2 \mathrm{H}), 7.11-7.01(\mathrm{~m}, 2 \mathrm{H}), 6.91-6.84(\mathrm{~m}, 1 \mathrm{H}), 6.84-$ $6.64(\mathrm{~m}, 1 \mathrm{H}), 4.83(\mathrm{~d}, J=6.6 \mathrm{~Hz}, 1 \mathrm{H}), 4.73-4.65(\mathrm{~m}, 1 \mathrm{H}), 3.01-2.76(\mathrm{~m}, 2 \mathrm{H}), 2.42-1.91(\mathrm{~m}, 2 \mathrm{H})$ ppm. ${ }^{13} \mathrm{C}$ NMR $\left(125 \mathrm{MHz}, \mathrm{CDCl}_{3}\right) \delta 189.3,153.5,134.9,134.8,129.7,129.3,129.2,127.7,121.5$, $121.4,117.0,115.1,74.0,45.0,25.2,24.0 \mathrm{ppm}$.

1a-4 was obtained as a colorless oil in 53\% yield ( $\mathrm{dr}=1: 1) .{ }^{1} \mathbf{H} \mathbf{N M R}\left(500 \mathrm{MHz}, \mathrm{CDCl}_{3}\right) \delta 7.12-7.01$ (m, 2H), $6.90-6.84(\mathrm{~m}, 1 \mathrm{H}), 6.80(\mathrm{dd}, J=15.3,8.2 \mathrm{~Hz}, 1 \mathrm{H}), 4.63-4.50(\mathrm{~m}, 1 \mathrm{H}), 4.41-4.23(\mathrm{~m}, 2 \mathrm{H})$, $3.96-3.72(\mathrm{~m}, 1 \mathrm{H}), 2.99-2.74(\mathrm{~m}, 2 \mathrm{H}), 2.23-2.01(\mathrm{~m}, 2 \mathrm{H}), 1.40-1.30(\mathrm{~m}, 3 \mathrm{H})$ ppm. ${ }^{13} \mathrm{C}$ NMR $(125$ $\left.\mathrm{MHz}_{\mathrm{CDCl}}\right) \delta 164.0,153.6,129.4,127.6,121.2,120.8,117.0,114.1,73.4,63.2,44.2,25.6,24.2$, $14.0 \mathrm{ppm}$.

1a was obtained as a white solid in $76 \%$ yield. ${ }^{1} \mathrm{H}$ NMR $\left(500 \mathrm{MHz}, \mathrm{CDCl}_{3}\right) \delta 7.13(\mathrm{t}, J=7.4 \mathrm{~Hz}, 1 \mathrm{H})$, $7.07(\mathrm{~d}, J=7.5 \mathrm{~Hz}, 1 \mathrm{H}), 6.93(\mathrm{t}, J=7.4 \mathrm{~Hz}, 1 \mathrm{H}), 6.88(\mathrm{~d}, J=8.2 \mathrm{~Hz}, 1 \mathrm{H}), 4.46$ (ddd, $J=10.7,5.4,2.3 \mathrm{~Hz}$, $1 \mathrm{H}), 4.06(\mathrm{~d}, J=5.5 \mathrm{~Hz}, 1 \mathrm{H}), 3.01-2.82(\mathrm{~m}, 2 \mathrm{H}), 2.34-2.27(\mathrm{~m}, 1 \mathrm{H}), 2.14-2.02(\mathrm{~m}, 1 \mathrm{H}) \mathrm{ppm} .{ }^{13} \mathrm{C}$ $\operatorname{NMR}\left(125 \mathrm{MHz}, \mathrm{CDCl}_{3}\right) \delta 152.9,129.6,128.2,122.2,120.6,117.2,110.5,73.0,29.3,25.0,23.7$ ppm. 


\section{Reactivity study of E1cB substrates}

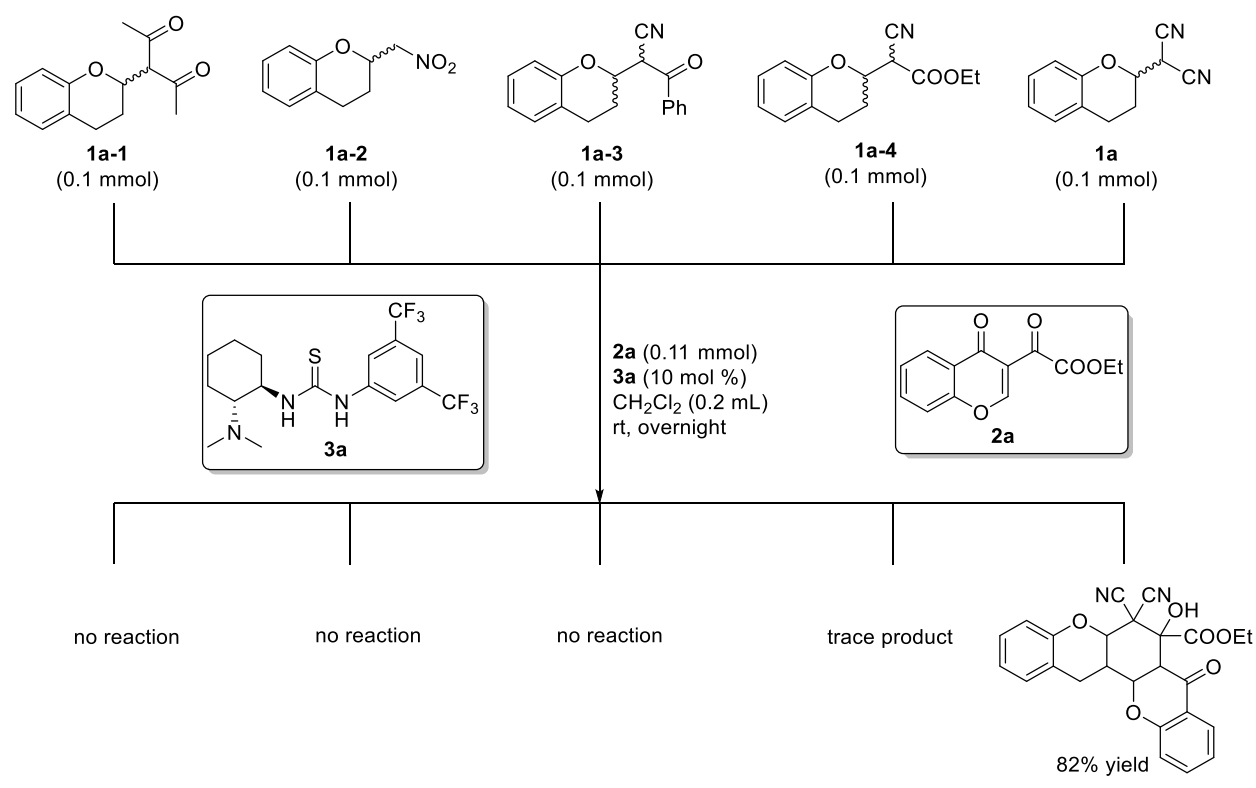

With these E1cB substrates in hand, we then studied their reactivities in the reaction with substrate $\mathbf{2 a}$ in the presence of Takemoto catalyst $\mathbf{3 a}$. All these reactions were performed by the same procedure: E1cB substrate $1(0.1 \mathrm{mmol}), \mathbf{2 a}(0.11 \mathrm{mmol})$ and catalyst $\mathbf{3 a}(10 \mathrm{~mol} \%)$ was dissolved in $\mathrm{CH}_{2} \mathrm{Cl}_{2}(0.2 \mathrm{~mL})$ at room temperature. The reaction was stirred overnight and detected by TLC until substrate 1 was consumed completely. As shown above, only substrate 1a could be catalyzed and give [3+3] cyclized product with $82 \%$ yield. Finally we chose $\mathbf{1 a}$ and $\mathbf{2 a}$ as model substrates to optimize reaction conditions. 


\section{Condition Optimization}

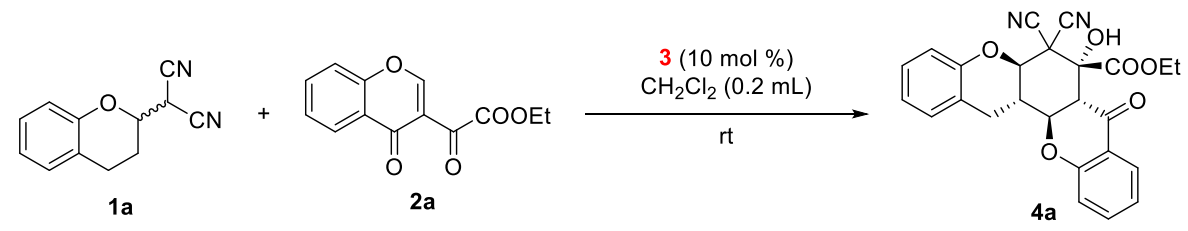

(DHQD) ${ }_{2}$ PYR, 90\%, 24\% ee (DHQD) 2 AQN, $86 \%, 17 \%$ ee (DHQD) ${ }_{2} \mathrm{PHAL}, 86 \%, 20 \%$ ee
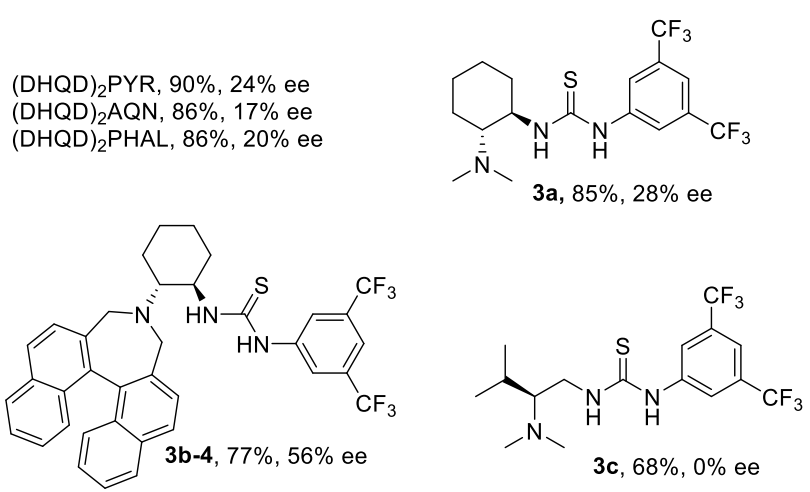<smiles>[R]c1ccc2nccc(C3NC(Nc4cc(C(F)(F)F)cc(C(F)(F)F)c4)=NN4C=C(C=C)CC34)c2c1</smiles>

3b, R = OMe, $77 \%, 32 \%$ ee $3 b-1, R=H, 72 \%, 25 \%$ ee
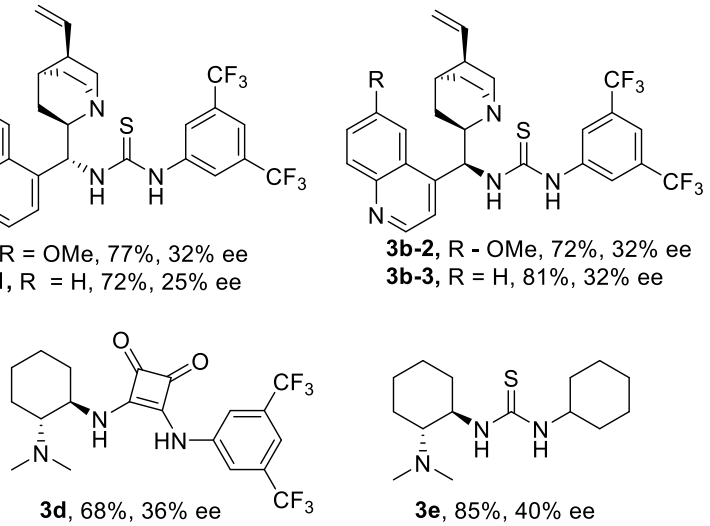

$3 e, 85 \%, 40 \%$ ee

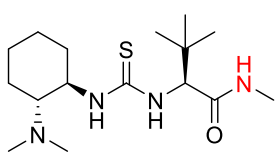

3f, $90 \%, 64 \%$ ee

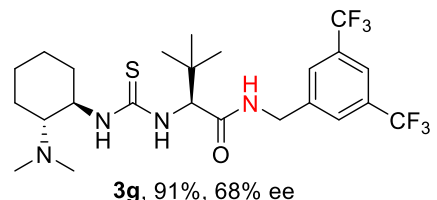

3g, $91 \%, 68 \%$ ee

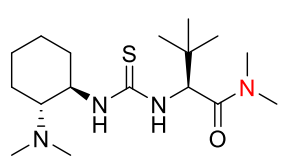

3h, $90 \%, 66 \%$ ee

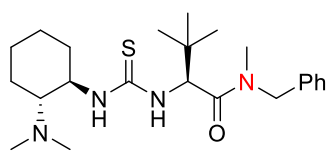

3i, $95 \%, 67 \%$ ee

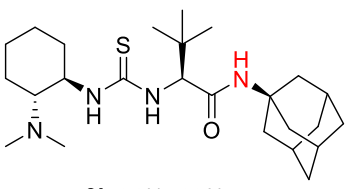

3j, $91 \%, 63 \%$ ee

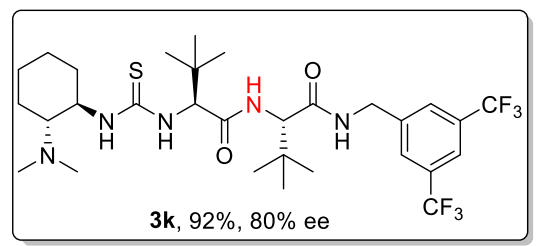

3k, $92 \%, 80 \%$ ee

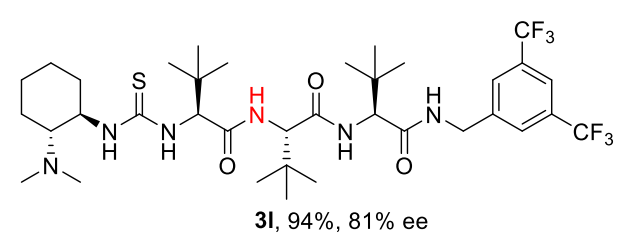

Table 1:

\begin{tabular}{cccccc}
\hline entry & $\mathbf{3}(\mathrm{mol} \%)$ & solvent $(\mathrm{mL})$ & $T(\mathrm{~h})$ & yield $(\%)^{b}$ & ee $(\%)^{c}$ \\
\hline 1 & $\mathbf{3 a}(10)$ & $\mathrm{CH}_{2} \mathrm{Cl}_{2}(0.2)$ & 2 & 85 & 28 \\
2 & $\mathbf{3 b}(10)$ & $\mathrm{CH}_{2} \mathrm{Cl}_{2}(0.2)$ & 4 & 77 & 32 \\
3 & $\mathbf{3 b - 1}(10)$ & $\mathrm{CH}_{2} \mathrm{Cl}_{2}(0.2)$ & 6 & 72 & 25 \\
4 & $\mathbf{3 b - 2}(10)$ & $\mathrm{CH}_{2} \mathrm{Cl}_{2}(0.2)$ & 4 & 72 & 32 \\
5 & $\mathbf{3 b - 3}(10)$ & $\mathrm{CH}_{2} \mathrm{Cl}_{2}(0.2)$ & 6 & 81 & 32 \\
6 & $\mathbf{3 b - 4}(10)$ & $\mathrm{CH}_{2} \mathrm{Cl}_{2}(0.2)$ & 16 & 77 & 56 \\
7 & $\mathbf{3 c}(10)$ & $\mathrm{CH}_{2} \mathrm{Cl}_{2}(0.2)$ & 4 & 68 & 0 \\
8 & $\mathbf{3 d}(10)$ & $\mathrm{CH}_{2} \mathrm{Cl}_{2}(0.2)$ & 4 & 68 & 36
\end{tabular}




\begin{tabular}{|c|c|c|c|c|c|}
\hline 9 & $3 \mathbf{e}(10)$ & $\mathrm{CH}_{2} \mathrm{Cl}_{2}(0.2)$ & 8 & 85 & 40 \\
\hline 10 & 3f $(10)$ & $\mathrm{CH}_{2} \mathrm{Cl}_{2}(0.2)$ & 10 & 90 & 64 \\
\hline 11 & $3 \mathbf{g}(10)$ & $\mathrm{CH}_{2} \mathrm{Cl}_{2}(0.2)$ & 2 & 91 & 68 \\
\hline 12 & $\mathbf{3 h}(10)$ & $\mathrm{CH}_{2} \mathrm{Cl}_{2}(0.2)$ & 4 & 90 & 66 \\
\hline 13 & $3 \mathbf{i}(10)$ & $\mathrm{CH}_{2} \mathrm{Cl}_{2}(0.2)$ & 4 & 95 & 67 \\
\hline 14 & $\mathbf{3 j}(10)$ & $\mathrm{CH}_{2} \mathrm{Cl}_{2}(0.2)$ & 2 & 91 & 63 \\
\hline 15 & $3 \mathbf{k}(10)$ & $\mathrm{CH}_{2} \mathrm{Cl}_{2}(0.2)$ & 4 & 92 & 80 \\
\hline 16 & $31(10)$ & $\mathrm{CH}_{2} \mathrm{Cl}_{2}(0.2)$ & 4 & 94 & 81 \\
\hline 17 & 3k (2) & $\mathrm{CH}_{2} \mathrm{Cl}_{2}(0.2)$ & 32 & 88 & 86 \\
\hline $18^{d}$ & $\mathbf{3 k}(2)$ & $\mathrm{CH}_{2} \mathrm{Cl}_{2}(0.2)$ & 36 & 88 & 87 \\
\hline $19^{d e}$ & $3 \mathbf{k}(1)$ & $\mathrm{CH}_{2} \mathrm{Cl}_{2}(1.0)$ & 10 & 86 & 93 \\
\hline $20^{d e}$ & $3 \mathbf{k}(0.5)$ & $\mathrm{CH}_{2} \mathrm{Cl}_{2}(1.0)$ & 36 & 79 & 91.5 \\
\hline $21^{d e}$ & $3 \mathbf{k}(0.2)$ & $\mathrm{CH}_{2} \mathrm{Cl}_{2}(1.0)$ & 68 & 78 & 89.5 \\
\hline $22^{d e}$ & $3 \mathbf{k}(1)$ & toluene (1.0) & 10 & 76 & 89 \\
\hline $23^{d e}$ & $3 \mathbf{k}(1)$ & MTBE (1.0) & 12 & 62 & 28 \\
\hline $24^{d e}$ & $3 \mathbf{k}(1)$ & $\mathrm{CHCl}_{3}(1.0)$ & 18 & 81 & 91 \\
\hline $25^{d e}$ & $3 \mathbf{k}(1)$ & benzene (1.0) & 16 & 80 & 78 \\
\hline $26^{d e}$ & 3k (1) & THF (1.0) & 8 & 84 & 2 \\
\hline $27^{d e}$ & $3 \mathbf{k}(1)$ & $\mathrm{MeCN}(1.0)$ & 4 & 78 & 0 \\
\hline $28^{d e}$ & 3k (1) & DCE (1.0) & 16 & 81 & 84 \\
\hline
\end{tabular}

aUnless otherwise noted, the reaction was conducted with $1 \mathbf{a}(0.1 \mathrm{mmol})$ and $\mathbf{2 a}(0.11 \mathrm{mmol}), \mathbf{3}(10 \mathrm{~mol} \%)$ in $\mathrm{CH}_{2} \mathrm{Cl}_{2}$ $(0.2 \mathrm{~mL})$ at $25^{\circ} \mathrm{C} .{ }^{b}$ Isolated yield. ${ }^{c}$ Determined by HPLC analysis on a chiral stationary phase. ${ }^{d}$ With $4 \AA \mathrm{MS}(20 \mathrm{mg}) .{ }^{e} \mathrm{At}$ $40^{\circ} \mathrm{C}$.

At the beginning, 3 different DHQD catalysts were used but gave products with poor ee. When Takemoto catalyst and some other thiourea tertiary amine catalysts were applied in this reaction, we could also get products in good yield but with moderate enantioselectivity (entries 1-9). A series of tert-leucine derived bifunctional amine-thiourea catalysts were then evaluated and gave products with better enantioselectivity (entries 10-16). These results motivated us to design and synthesize 3k and $\mathbf{3}$ which contain dipeptide and tripeptide moieties. It was interesting that the cascade reaction proceeded smoothly in the presence of $\mathbf{3 k}$ or $\mathbf{3} \mathbf{l}$ and afforded product in high yield with good enantioselectivity (entries 15 and 16). Lower catalyst loading, addition of $4 \AA$ MS and higher temperature made the reaction work better (entries 17-19). But much lower catalyst loading made result worse (entries 20 and 21). We had screened different solvents but no better result was 
obtained (entries 22-28). Finally, we chose entry 19 as the optimal reaction condition.

\section{Preparation of catalyst $3 \mathrm{k}$ and $3 \mathbf{I}^{1}$}
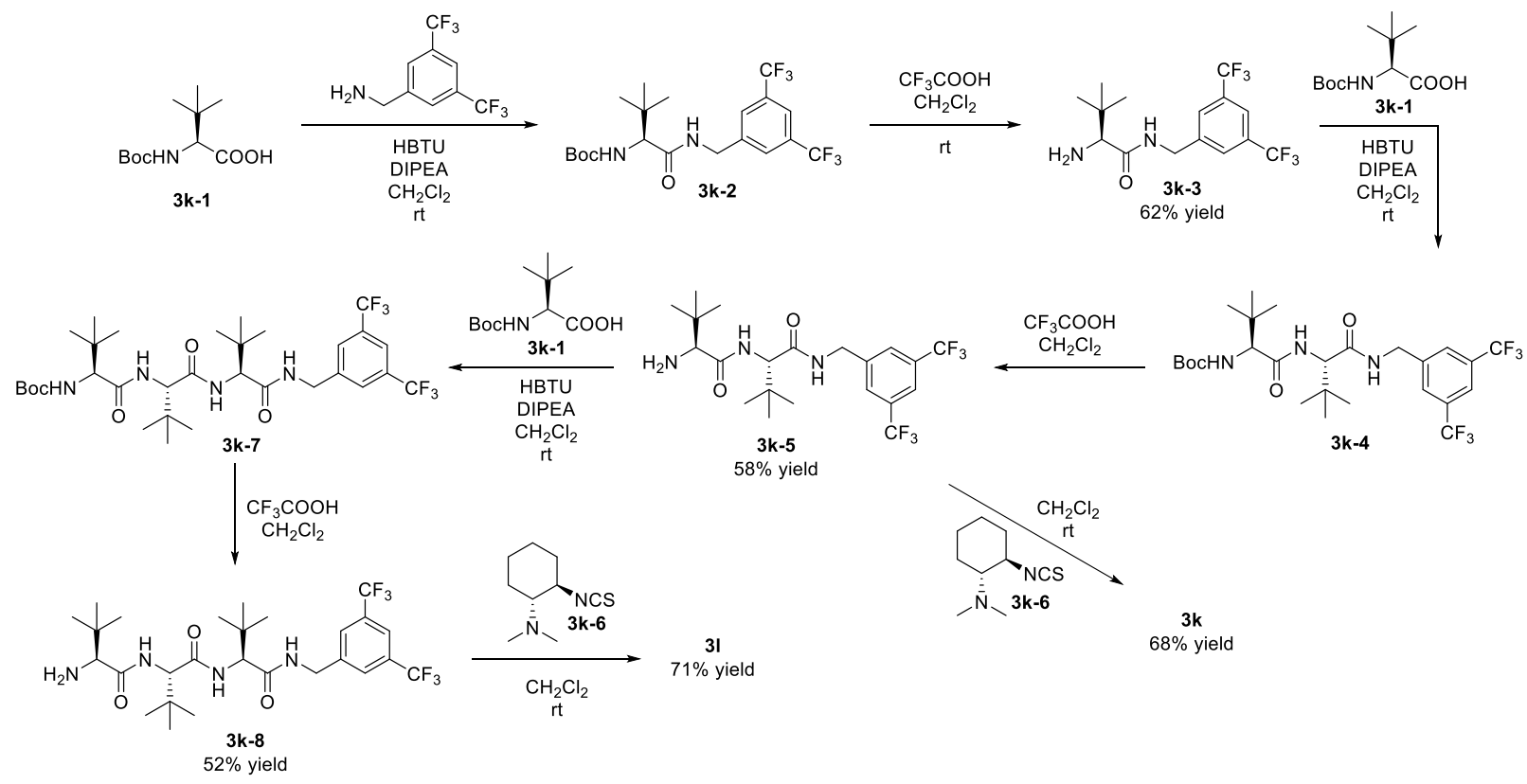

To a solution of $3 \mathbf{k - 1}(5 \mathrm{mmol}), \mathrm{HBTU}(5.5 \mathrm{mmol})$ in $\mathrm{CH}_{2} \mathrm{Cl}_{2}(30 \mathrm{~mL})$, DIPEA $(6 \mathrm{mmol})$ and 3,5-bis(trifluoromethyl)benzylamine $(5.5 \mathrm{mmol})$ was added at room temperature. The reaction was stirred at room temperature for $6 \mathrm{~h}$ and quenched by $1 \mathrm{M} \mathrm{HCl}(20 \mathrm{~mL})$. The Reaction was extracted with AcOEt. The organic phase was then washed with sat. $\mathrm{NaHCO}_{3}$, dried over $\mathrm{Na}_{2} \mathrm{SO}_{4}$ and concentrated to give $\mathbf{3 k - 2}$ as yellow oil without purification.

To a solution of $3 \mathbf{k - 2}(3 \mathrm{mmol})$ in $\mathrm{CH}_{2} \mathrm{Cl}_{2}(10 \mathrm{~mL}), \mathrm{CF}_{3} \mathrm{COOH}(2 \mathrm{~mL})$ was added dropwise and the reaction was stirred at room temperature for $2 \mathrm{~h}$. It was quenched by addition of aqueous $\mathrm{Na}_{2} \mathrm{CO}_{3}$ until $\mathrm{pH}$ was 8-9. Then separated organic phase was washed with brine, dried over $\mathrm{Na}_{2} \mathrm{SO}_{4}$ and concentrated to give $\mathbf{3 k - 3}$ as yellow oil in $62 \%$ yield for 2 steps after purified by flash column chromatography on silica gel.

Compound 3k-5 and 3k-8 were synthesized by the same method above.

Compound 3k-5 or 3k-8 $(0.5 \mathrm{mmol})$ and $3 \mathbf{k}-6(0.5 \mathrm{mmol})$ was dissolved in $\mathrm{CH}_{2} \mathrm{Cl}_{2}(2 \mathrm{~mL})$. The reaction was performed at $25{ }^{\circ} \mathrm{C}$ for $24 \mathrm{~h}$ until completed. Catalysts $3 \mathbf{k}$ was obtained as white solid 
in $68 \%$ yield and $\mathbf{3 l}$ was obtained as white solid in $68 \%$ yield after purified by flash column chromatography on silica gel.

3k ${ }^{1} \mathrm{H}-\mathrm{NMR}\left(500 \mathrm{MHz}, \mathrm{CDCl}_{3}\right) \delta 7.77-7.72(\mathrm{~m}, 3 \mathrm{H}), 7.67(\mathrm{~s}, 1 \mathrm{H}), 7.08(\mathrm{~s}, 1 \mathrm{H}), 6.93(\mathrm{~d}, J=7.8 \mathrm{~Hz}, 1 \mathrm{H})$, $4.57-4.39(\mathrm{~m}, 3 \mathrm{H}), 4.36(\mathrm{~d}, J=8.5 \mathrm{~Hz}, 1 \mathrm{H}), 2.69(\mathrm{~s}, 1 \mathrm{H}), 2.37-2.21(\mathrm{~m}, 7 \mathrm{H}), 1.92-1.78(\mathrm{~m}, 1 \mathrm{H})$, $1.70(\mathrm{~d}, J=11.2 \mathrm{~Hz}, 1 \mathrm{H}), 1.33-1.15(\mathrm{~m}, 5 \mathrm{H}), 1.00(\mathrm{~d}, J=18 \mathrm{~Hz}, 18 \mathrm{H}) \mathrm{ppm} .{ }^{13} \mathrm{C} \mathrm{NMR}\left(125 \mathrm{MHz}, \mathrm{CDCl}_{3}\right)$ $\delta 183.0,171.9,171.4,141.8,132.0,131.7,128.1,124.5,121.3,67.9,67.1,61.5,55.2,42.5,40.0$, $34.5,34.2,33.3,29.9,27.5,27.1,25.0,24.8,22.1 \mathrm{ppm}$.

3I ${ }^{1} \mathrm{H}$ NMR $\left(500 \mathrm{MHz}, \mathrm{CDCl}_{3}\right) \delta 7.71(\mathrm{~s}, 3 \mathrm{H}), 7.37(\mathrm{~s}, 1 \mathrm{H}), 7.13(\mathrm{~s}, 1 \mathrm{H}), 6.79(\mathrm{~d}, J=7.4 \mathrm{~Hz}, 1 \mathrm{H}), 6.73(\mathrm{~d}$, $J=6.0 \mathrm{~Hz}, 1 \mathrm{H}), 4.64(\mathrm{~d}, J=7.4 \mathrm{~Hz}, 1 \mathrm{H}), 4.55-4.44(\mathrm{~m}, 2 \mathrm{H}), 4.31(\mathrm{~d}, J=9.0 \mathrm{~Hz}, 1 \mathrm{H}), 4.23(\mathrm{~d}, J=8.0 \mathrm{~Hz}$, 1H), $4.09(\mathrm{~s}, 1 \mathrm{H}), 2.80(\mathrm{~s}, 1 \mathrm{H}), 2.40(\mathrm{~s}, 6 \mathrm{H}), 2.30(\mathrm{~d}, J=12 \mathrm{~Hz}, 1 \mathrm{H}), 1.98-1.80(\mathrm{~m}, 2 \mathrm{H}), 1.72(\mathrm{~d}, J=$ $12.0 \mathrm{~Hz}, 1 \mathrm{H}), 1.37-1.23(\mathrm{~m}, 5 \mathrm{H}), 0.96(\mathrm{~m}, 27 \mathrm{H}) \mathrm{ppm} .{ }^{13} \mathrm{C}$ NMR (125 MHz, $\left.\mathrm{d}_{6}-\mathrm{DMSO}\right) \delta$ 182.8, 171.9, $170.8,170.5,141.1,131.8,131.5,127.8,124.3,122.1,121,2,67.0,66.6,61.7,61.0,54.7,42.4,39.9$, $34.4,34.3,34.2,32.9,29.7,27.1,26.8,26.7,24.6,24.5,22.4$ ppm. 


\section{Synthesis of Substrates}

\section{Preparation of substituted substrates 1a-g}

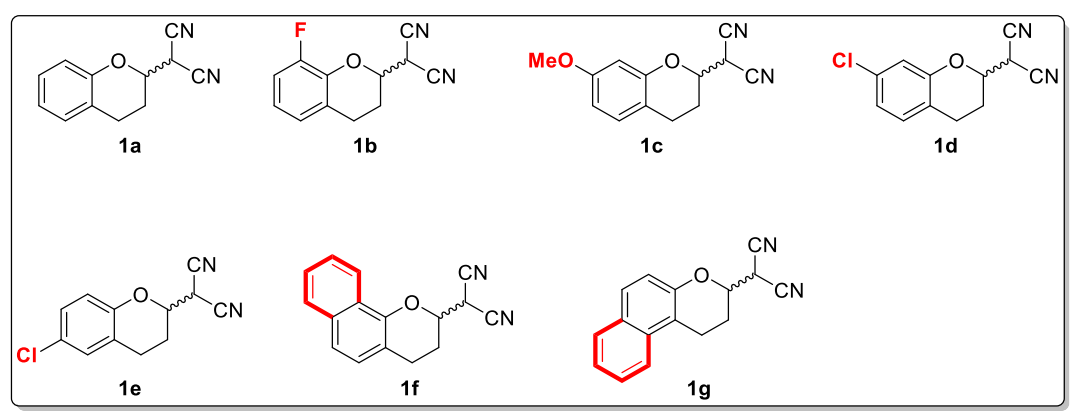

\section{Synthesis of substrates 1a}

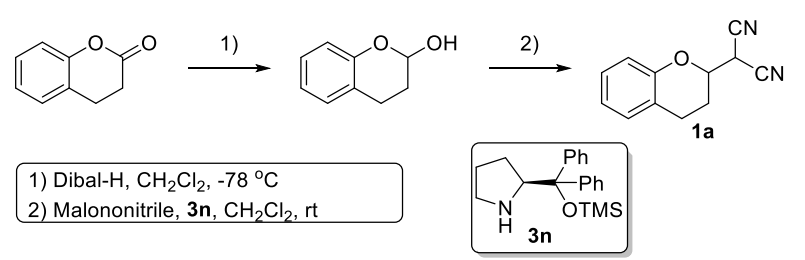

\section{General procedure 1:}

Step 1: Diisobutylaluminium hydride $(1.5 \mathrm{M}, 0.8 \mathrm{~mL})$ was slowly added into a solution of lactone (150 mg, $1 \mathrm{mmol}$ ) in dry $\mathrm{CH}_{2} \mathrm{Cl}_{2}(2 \mathrm{~mL})$ at $-78{ }^{\circ} \mathrm{C}$. The reaction was performed at $-78{ }^{\circ} \mathrm{C}$ for $1.5 \mathrm{~h}$ and quenched by $\mathrm{MeOH}(1 \mathrm{~mL})$. The reaction was diluted with $\mathrm{CH}_{2} \mathrm{Cl}_{2}$ and $0.5 \mathrm{~mL}$ water was added. The reaction was stirred at room temperature for $30 \mathrm{~min}$ when flocculent precipitation occurred. The crude mixture was filtered through kieselguhr, the filtrate was concentrated, and purified by flash column chromatography on silica gel to give lactol intermediate.

Step 2: Lactol (0.5 mmol) and malononitrile (0.5 mmol) were dissolved in $\mathrm{CH}_{2} \mathrm{Cl}_{2}(1 \mathrm{~mL}) .3 \mathrm{n}(5 \mathrm{~mol} \%)$ was added and the reaction was stirred at room temperature until lactol was consumed completely. The reaction was purified by flash column chromatography on silica gel to give 1a in $70 \%$ yield. 


\section{Synthesis of substrates $1 \mathrm{~b}-\mathrm{e}$ :}

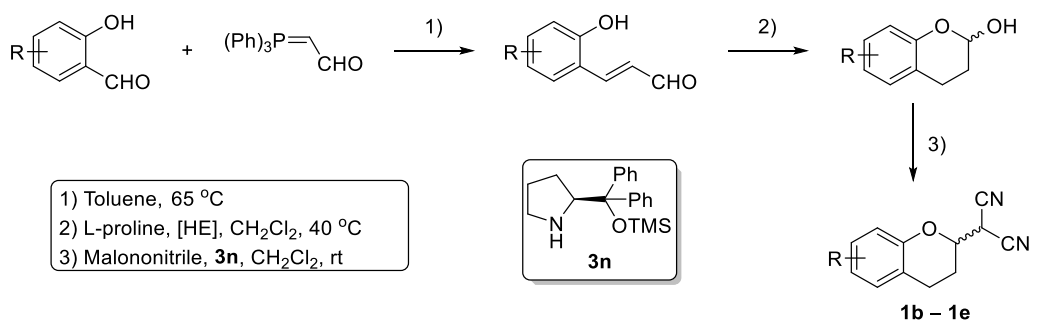

Substrates $\mathbf{1 b}$-e were synthesized from the commercial salicylaldehydes by general procedure $\mathbf{2}$.

\section{General procedure 2:}

Step 1: Salicylaldehyde $(5.0 \mathrm{mmol})$ and witting reagent $(5.5 \mathrm{mmol})$ were dissolved in toluene $(10$ $\mathrm{mL}$ ). The reaction was performed at $65^{\circ} \mathrm{C}$ for $24 \mathrm{~h}$. The solvent was removed under vacuum and the crude products were purified by flash column chromatography on silica gel to give the corresponding pure 2-hydroxycinnamaldehyde.

Step 2: 2-Hydroxycinnamaldehyde $(1.0 \mathrm{mmol})$, diethyl 1,4-dihydro-2,6-dimethyl-3,5pyridinedicarboxylate ( $\mathrm{HE}, 1.1 \mathrm{mmol})$ and L-proline $(10 \mathrm{~mol} \%)$ were dissolved in $\mathrm{CH}_{2} \mathrm{Cl}_{2}(1 \mathrm{~mL})$. The reaction was performed at $40{ }^{\circ} \mathrm{C}$ until starting material consumed completely. The lactol was obtained after purified by flash column chromatography on silica gel.

Step 3: Substrates $\mathbf{1 b}$-e were obtained by the method of step 2 in General procedure 1. 


\section{Synthesis of substrates $1 \mathrm{f}$ and $1 \mathrm{~g}$}

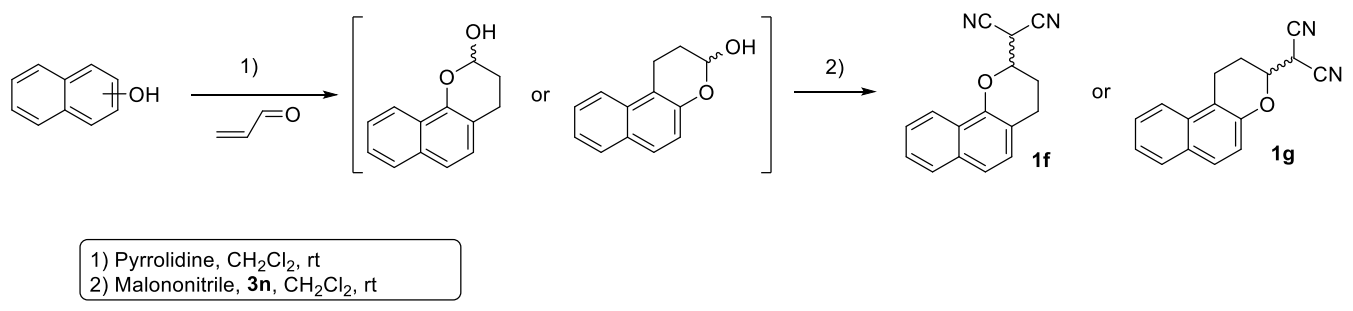

\section{General procedure 3:}

Step 1: Naphthol or 2-naphthol $(1.0 \mathrm{mmol})$ and acrolein $(1.5 \mathrm{mmol})$ were dissolved in $\mathrm{CH}_{2} \mathrm{Cl}_{2}(1 \mathrm{~mL})$, then pyrrolidine $(10 \mathrm{~mol} \%)$ was added. The reaction was performed at room temperature until naphthol was consumed completely. Lactols were obtained after purified by flash column chromatography on silica gel.

Step 2: Substrates $\mathbf{1 f}$ and $\mathbf{1 g}$ were obtained by the method of step 2 in General procedure $\mathbf{1 .}$ 


\section{Preparation of substituted substrates $2 a-q$}

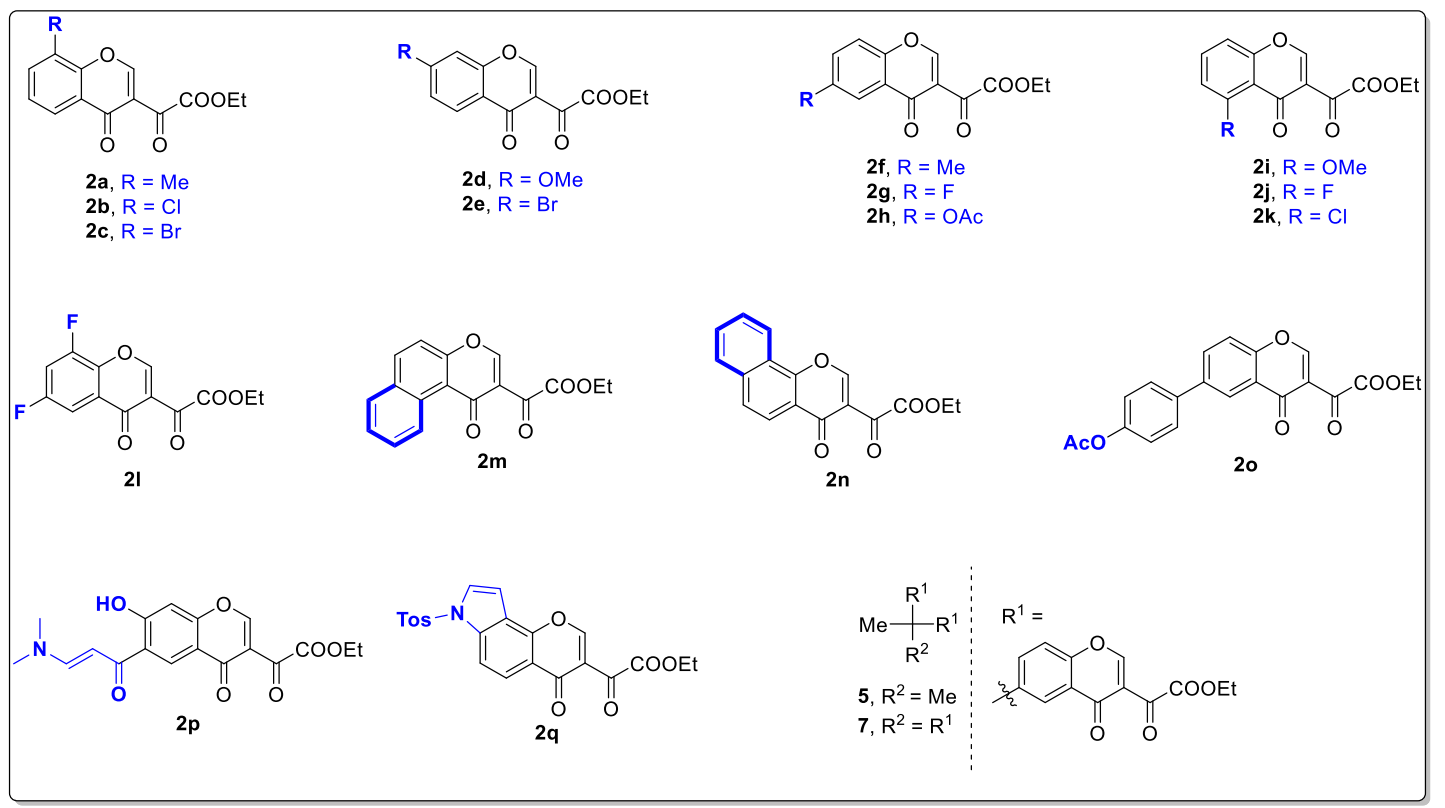

\section{Synthesis of substrates $2 a-g$ and $2 i-n$}

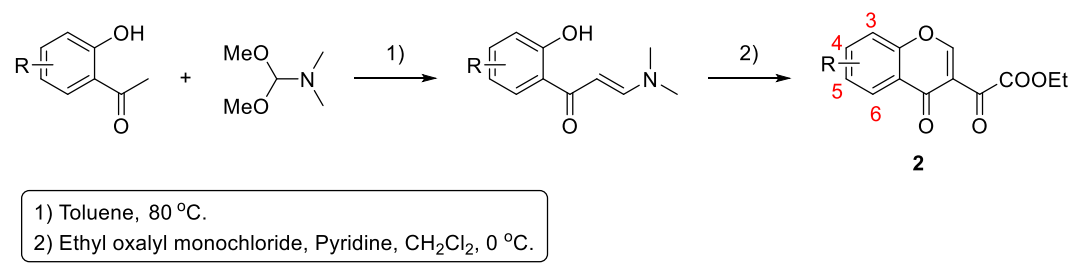

Substrates $\mathbf{2 a - g}$ and $\mathbf{2} \mathbf{i}-\mathbf{n}$ were synthesized from commercial $o$-hydroxyacetophenone by general procedure 4.

\section{General procedure 4:2 3}

Step 1: o-Hydroxyacetophenone $(2 \mathrm{mmol})$ was dissolved in toluene $(2 \mathrm{~mL})$ and $\mathrm{N}, \mathrm{N}$-Dimethylformamide dimethyl acetal (DMF-DMA) $(4.0 \mathrm{mmol})$ was added at room temperature. The reaction was allowed to stir at $80^{\circ} \mathrm{C}$ until o-hydroxyacetophenone was consumed completely as detected by TLC. The reaction mixture was filtered or purified by flash column chromatography on silica gel to afford o-hydroxyarylenaminones.

2 S. Mkrtchyan, V. O. Iaroshenko, S. Dudkin, A. Gevorgyan, M. Vilches-Herrera, G. Ghazaryan, D. M. Volochnyuk, D. Ostrovskyi, Z. Ahmed, A. Villinger, V. Y. Sosnovskikh, P. Langer, Org. Biomol. Chem. 2010, 8, 5280.

3 Akram, M. O.; Bera, S.; Patil, N. T. Chem. Commun. 2016, 52, 12306. 
Step 2: Dry pyridine $(2 \mathrm{mmol})$ and ethyl oxalyl monochloride $(1.5 \mathrm{mmol})$ was successively added into a solution of $o$-hydroxyarylenaminones $(1 \mathrm{mmol})$ in dry $\mathrm{CH}_{2} \mathrm{Cl}_{2}(2 \mathrm{~mL})$ at $0{ }^{\circ} \mathrm{C}$. The reaction was allowed at $0{ }^{\circ} \mathrm{C}$ for $30 \mathrm{~min}$. Then $1 \mathrm{M} \mathrm{HCl}$ and $\mathrm{CH}_{2} \mathrm{Cl}_{2}$ was added and the separated organic layer was dried and concentrated. After recrystallization (ethyl acetate $/ n$-hexane $=1 / 1$ ) or purified by flash column chromatography on silica gel, substituted substrates $\mathbf{2}$ were obtained. 


\section{Synthesis of substrate $\mathbf{2 h}$ and $\mathbf{2 0}$}
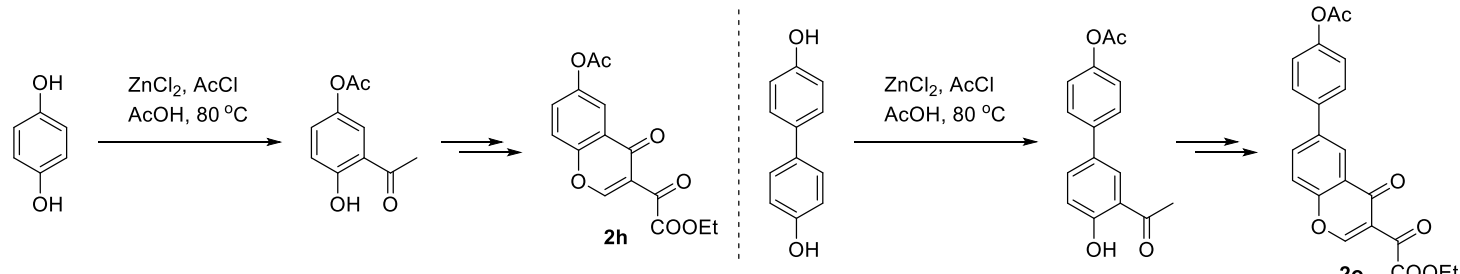

2o COOEt

\section{General procedure 5:}

Hydroquinone ( $2 \mathrm{mmol}$ ) or 4,4'-Biphenol $(2 \mathrm{mmol})$ and $\mathrm{ZnCl}_{2}(8 \mathrm{mmol})$ were dissolved in $\mathrm{AcOH}(1 \mathrm{~mL})$ in a sealing tube. Then $\mathrm{AcCl}(4.8 \mathrm{mmol})$ was added dropwise at room temperature. The reaction was allowed to stir at $80{ }^{\circ} \mathrm{C}$ for $1 \mathrm{~h}$. The reaction was diluted with ethyl acetate and filtrated through kieselguhr. The crude filter was concentrated and purified by flash column chromatography on silica gel to afford corresponding substituted $o$-hydroxyacetophenone.

Substrates $\mathbf{2} \mathbf{h}$ and $\mathbf{2 0}$ were obtained from the corresponding substituted $\boldsymbol{o}$-hydroxyacetophenone by the method of General procedure 4. 


\section{Synthesis of substrate $2 p$}

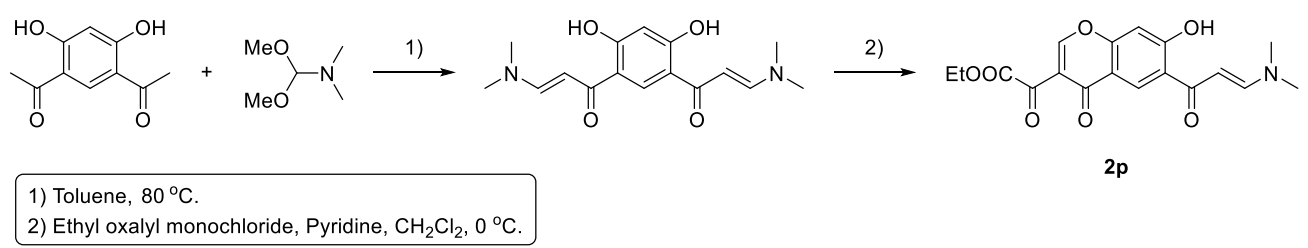

\section{General procedure 6:}

Step 1: 4,6-Diacetylresorcinol (2 mmol) was dissolved in toluene $(2 \mathrm{~mL}$ ) and DMF-DMA (8.0 mmol) was added at room temperature. The reaction was allowed to stir at $80{ }^{\circ} \mathrm{C}$ until 4,6-diacetylresorcinol and single-addition-product were consumed completely as detected by TLC. The yellow precipitation was filtered and used in the next step without purification.

Step 2: The obtained solid (1 mmol) was dissolved in dry $\mathrm{CH}_{2} \mathrm{Cl}_{2}(2 \mathrm{~mL})$. Dry pyridine $(4 \mathrm{mmol})$ and ethyl oxalyl monochloride $\left(3 \mathrm{mmol}\right.$ ) was successively added at $0{ }^{\circ} \mathrm{C}$. The reaction was allowed at 0 ${ }^{\circ} \mathrm{C}$ for 30 min when substrate was consumed completely. Then $1 \mathrm{M} \mathrm{HCl}$ and $\mathrm{CH}_{2} \mathrm{Cl}_{2}$ was added and the separated organic layer was dried and concentrated. After purified by flash column chromatography on silica gel, the single-cyclic-product $2 p$ was obtained. 


\section{Synthesis of substrate $2 q$}

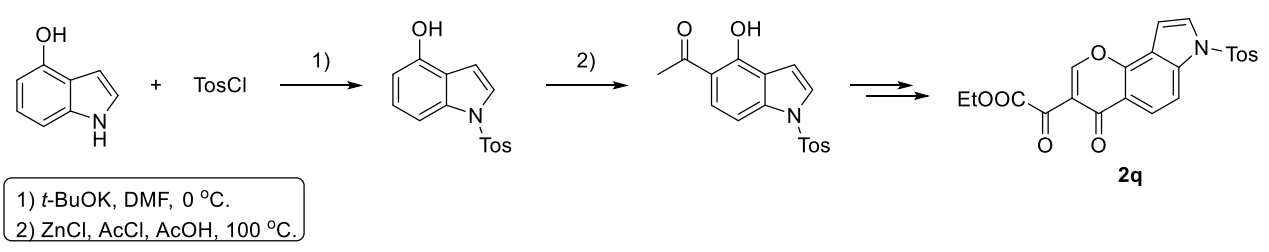

\section{General procedure 7:}

Step 1:4 4-Hydroxyindole (5 mmol) was dissolved in DMF $(5 \mathrm{~mL})$ and $t$-BuOK $(10 \mathrm{mmol})$ was added at $0{ }^{\circ} \mathrm{C} .10$ minutes later, tosyl chloride (Tos $\left.\mathrm{Cl}, 5.5 \mathrm{mmol}\right)$ was added. The reaction was performed at $0{ }^{\circ} \mathrm{C}$ for $1 \mathrm{~h}$. The crude mixture was extracted with ethyl acetate/ $\mathrm{H}_{2} \mathrm{O}$. The organic layer was dried with $\mathrm{Na}_{2} \mathrm{SO}_{4}$ and concentrated. After purified by flash column chromatography on silica gel, tosylation-product was obtained in $80 \%$ yield.

Step 2: The tosylation-product $(3 \mathrm{mmol})$ and $\mathrm{ZnCl}_{2}(9 \mathrm{mmol})$ were dissolved in $\mathrm{AcOH}(2 \mathrm{~mL})$ in a sealing tube. Then $\mathrm{AcCl}(3.6 \mathrm{mmol})$ was added dropwise at room temperature. The reaction was allowed to stir at $100^{\circ} \mathrm{C}$ for $1 \mathrm{~h}$. The reaction was diluted with ethyl acetate and filtrated through kieselguhr. The crude filter was concentrated and purified by flash column chromatography on silica gel to afford corresponding substituted o-hydroxyacetophenone in $73 \%$ yield.

Substrates $\mathbf{2 q}$ was obtained from the corresponding substituted $o$-hydroxyacetophenone by the method of General procedure 4.

\footnotetext{
${ }^{4}$ Wada, Y.; Harayama, Y.; Kamimura, D.; Yoshida, M.; Shibata, T.; Fujiwara, F.; Morimoto, K.; Fujioka, H.; Kita, Y. Org. Biomol. Chem. 2011, 9, 4959.
} 


\section{Scope of Substrates}

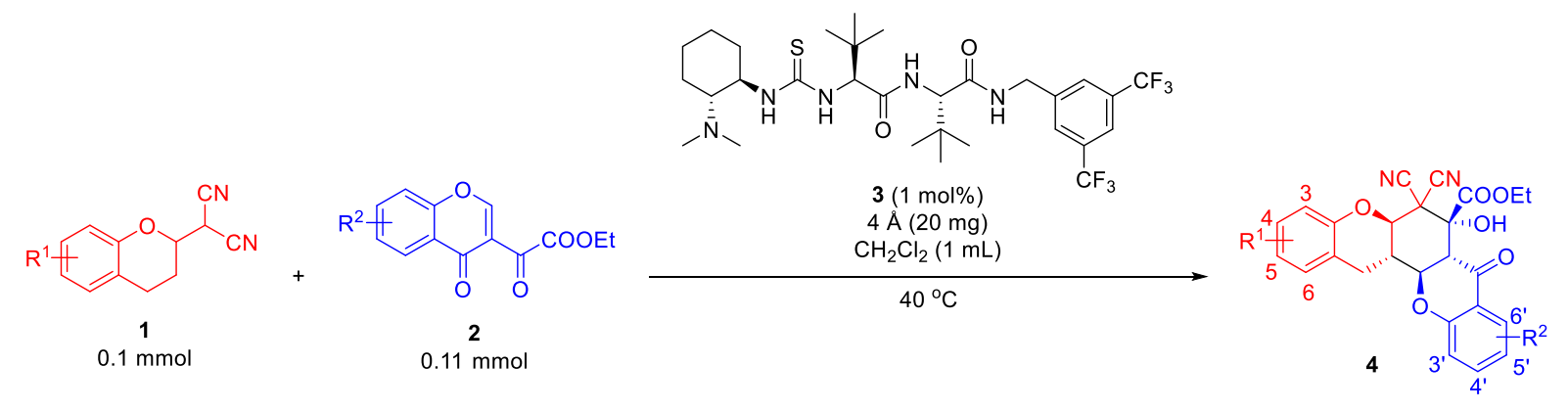

4a, $\mathrm{R}^{1}=\mathrm{H}, \mathrm{R}^{2}=\mathrm{H}, 86 \%, 93 \%$ ee 4b, $R^{1}=3-F, R^{2}=H, 78 \%, 97 \%$ ee 4c, $R^{1}=4-O M e, R^{2}=H, 84 \%, 93 \%$ ee $4 d, R^{1}=4-C l, R^{2}=H, 84 \%, 96 \%$ ee $4 e, R^{1}=5-C l, R^{2}=H, 82 \%, 92 \%$ ee 4f, $R^{1}=H, R^{2}=3 '-M e, 87 \%, 99.5 \%$ ee 4g, $R^{1}=H, R^{2}=3^{\prime}-C l, 82 \%, 99.5 \%$ ee $4 h, R^{1}=H, R^{2}=3^{\prime}-B r, 84 \%, 99.8 \%$ ee $4 i, R^{1}=H, R^{2}=4^{\prime}-$ OMe, $84 \%, 92 \%$ ee $4 j, R^{1}=H, R^{2}=4^{\prime}-B r, 88 \%, 94 \%$ ee 4k, $R^{1}=H, R^{2}=5^{\prime}-M e, 87 \%, 92 \%$ ee 4I, $R^{1}=H, R^{2}=5 '-F, 74 \%, 95 \%$ ee $4 m, R^{1}=H, R^{2}=5^{\prime}-O A c, 86 \%, 90 \%$ ee $4 n, R^{1}=H, R^{2}=6^{\prime}-O M e, 95 \%, 92 \%$ ee 4o, $R^{1}=H, R^{2}=6^{\prime}-F, 82 \%, 92 \%$ ee $4 p, R^{1}=H, R^{2}=6 '-C l, 79 \%, 96 \%$ ee $4 q, R^{1}=H, R^{2}=3^{\prime}-F, 5^{\prime}-F, 90 \%, 95 \%$ ee<smiles>CCOC(=O)[C@]1(O)[C@@H]2C(=O)c3ccc4c(ccn4[13CH3])c3O[C@H]2[C@@H]2Cc3ccccc3O[C@H]2C1(C)C#N</smiles>
4x, $91 \%, 91 \%$ ee<smiles>CCOC(=O)[C@]1(O)[C@@H]2C(=O)c3ccccc3O[C@H]2[C@@H]2Cc3c(ccc4ccccc34)O[C@H]2C1(C)C#N</smiles><smiles>CCO[C@H](O)[C@@]1(O)[C@@H]2C(=O)c3ccccc3O[C@H]2[C@@H]2Cc3ccc4ccccc4c3O[C@H]2C1(C)C</smiles><smiles>CCOC(=O)[C@]1(O)[C@H]2C(=O)c3ccc4ccccc4c3O[C@H]2[C@@H]2Cc3ccccc3OC2C1(C)C</smiles>

4t, $83 \%, 98 \%$ ee

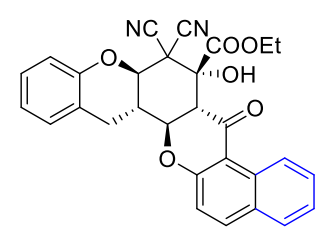

$4 u, 81 \%, 87 \%$ ee

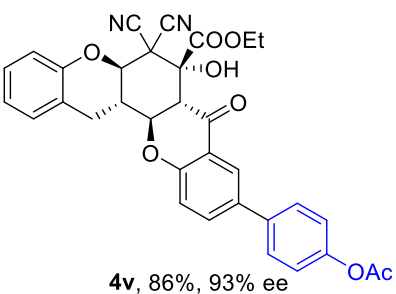

$4 v, 86 \%, 93 \%$ ee

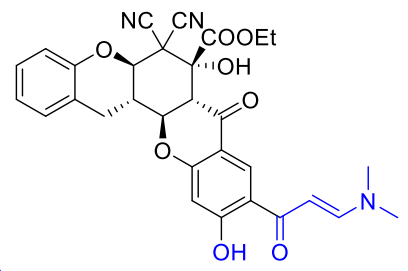

$4 w, 79 \%, 98 \%$ ee

General procedure 8: Substrates $1(0.1 \mathrm{mmol}), 2(0.11 \mathrm{mmol})$ and $4 \AA \mathrm{MS}(20 \mathrm{mg})$ were dissolved in $\mathrm{CH}_{2} \mathrm{Cl}_{2}(1 \mathrm{~mL})$ followed by addition of $3(0.65 \mathrm{mg}, 1 \mathrm{~mol} \%)$. The reaction was stirred at $40{ }^{\circ} \mathrm{C}$ overnight until substrate 1 was consumed completely. The reaction was purified by flash column chromatography on silica gel and products $4 \mathbf{a}-\mathbf{x}$ were obtained.

General procedure 9: 1a (22 mg, $0.11 \mathrm{mmol}), 5(27 \mathrm{mg}, 0.05 \mathrm{mmol})$ and $4 \AA \mathrm{MS}(20 \mathrm{mg})$ were dissolved in $\mathrm{CH}_{2} \mathrm{Cl}_{2}(1 \mathrm{~mL})$ followed by addition of $3(0.65 \mathrm{mg}, 1 \mathrm{~mol} \%)$. The reaction was stirred at $40{ }^{\circ} \mathrm{C}$ for $10 \mathrm{~h}$ until substrate 5 was consumed completely. The reaction was purified by flash column chromatography on silica gel and products 6 were obtained.

General procedure 10: 1a (22 mg, $0.11 \mathrm{mmol}), 7(25 \mathrm{mg}, 0.033 \mathrm{mmol})$ and $4 \AA \mathrm{MS}$ (20 mg) were 
dissolved in $\mathrm{CH}_{2} \mathrm{Cl}_{2}(1 \mathrm{~mL})$ followed by addition of $3(0.65 \mathrm{mg}, 1 \mathrm{~mol} \%)$. The reaction was stirred at $40{ }^{\circ} \mathrm{C}$ for $13 \mathrm{~h}$. The reaction was purified by flash column chromatography on silica gel and product 8 was obtained. 


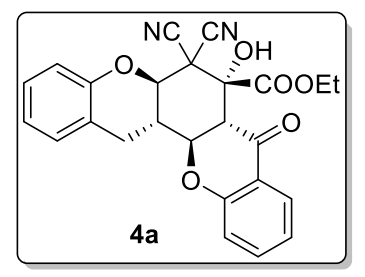

4a was obtained as a white solid (38 $\mathrm{mg}, 86 \%$ ) by General procedure $9 .{ }^{1} \mathbf{H}$ NMR (500 MHz, d6-DMSO) $\delta 7.92(\mathrm{~s}, 1 \mathrm{H}), 7.79(\mathrm{dd}, J=7.8,1.6 \mathrm{~Hz}, 1 \mathrm{H}), 7.70-$ $7.64(m, 1 H), 7.26(d, J=7.5 \mathrm{~Hz}, 1 \mathrm{H}), 7.22-7.13(m, 3 \mathrm{H}), 7.02-6.95(m, 2 \mathrm{H})$, $4.76(\mathrm{~d}, J=10.9 \mathrm{~Hz}, 1 \mathrm{H}), 4.60(\mathrm{dd}, J=12.9,10.2 \mathrm{~Hz}, 1 \mathrm{H}), 4.49-4.30(\mathrm{~m}, 2 \mathrm{H})$, $3.85(\mathrm{~d}, J=13.0 \mathrm{~Hz}, 1 \mathrm{H}), 3.34(\mathrm{dd}, J=16.2,5.1 \mathrm{~Hz}, 2 \mathrm{H}), 3.08(\mathrm{dd}, J=16.3,11.5 \mathrm{~Hz}, 1 \mathrm{H}), 2.66-2.56$ (m, 1H), $1.32(\mathrm{t}, J=7.1 \mathrm{~Hz}, 3 \mathrm{H}) \mathrm{ppm} .{ }^{13} \mathrm{C}$ NMR (125 MHz, $\mathrm{d}_{6}$-DMSO) $\delta 187.7,168.0,160.5,152.2$ $137.1,129.7,127.8,126.8,122.2,122.0,120.5,120.0,118.0,116.2,112.0,111.3,77.0,76.5,74.5$, 62.8, 51.2, 47.7, 37.6, 26.4, 13.8 ppm. HRMS: [M-H] calcd. For $\mathrm{C}_{25} \mathrm{H}_{19} \mathrm{~N}_{2} \mathrm{O}_{6}{ }^{-}$443.1249; found: 443.1250. $[\alpha]_{D}{ }^{20}-140\left(c=0.25\right.$ in $\left.\mathrm{CHCl}_{3}\right)$. The enantiomeric excess was determined by HPLC analysis on Daicel Chiralpak IC column [n-hexane/i-PrOH $=80 / 20,1 \mathrm{~mL} / \mathrm{min}], \lambda=220 \mathrm{~nm}, t_{\text {minor }}=8.00 \mathrm{~min}$, $t_{\text {major }}=9.76 \min , \boldsymbol{e e}=\mathbf{9 3} \%$.

Ethyl $(5 a R, 7 R, 7 a R, 13 a S, 13 b S)-6,6-d i c y a n o-4-f l u o r o-7-h y d r o x y-8-o x o-5 a, 6,7,7 a, 8,13 a, 13 b, 14-$ octahydrochromeno[2,3-a]xanthene-7-carboxylate (4b)

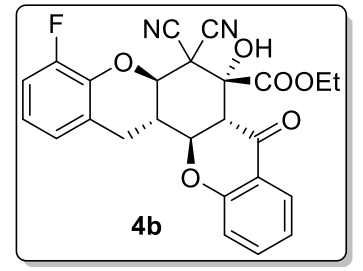

4b was obtained as a white solid (36 mg, 78\%) by General procedure $\mathbf{9} . \mathbf{1 H}$ NMR $\left(500 \mathrm{MHz}, \mathrm{d}_{6}-\mathrm{DMSO}\right) \delta 7.97(\mathrm{~s}, 1 \mathrm{H}), 7.79(\mathrm{~d}, J=7.2 \mathrm{~Hz}, 1 \mathrm{H}), 7.67(\mathrm{t}, J=$ $7.2 \mathrm{~Hz}, 1 \mathrm{H}), 7.20-7.11(\mathrm{~m}, 3 \mathrm{H}), 7.10(\mathrm{~d}, J=7.5 \mathrm{~Hz}, 1 \mathrm{H}), 7.02-6.94(\mathrm{~m}, 1 \mathrm{H})$ $4.88(\mathrm{~d}, J=10.9 \mathrm{~Hz}, 1 \mathrm{H}), 4.60(\mathrm{dd}, J=12.6,10.4 \mathrm{~Hz}, 1 \mathrm{H}), 4.48-4.33(\mathrm{~m}, 2 \mathrm{H})$, $3.86(\mathrm{~d}, J=12.9 \mathrm{~Hz}, 1 \mathrm{H}), 3.39(\mathrm{dd}, J=16.5,4.6 \mathrm{~Hz}, 1 \mathrm{H}), 3.12(\mathrm{dd}, J=16.2,11.7 \mathrm{~Hz}, 1 \mathrm{H}), 2.70-2.60$ (m, 1H), $1.32(\mathrm{t}, J=7.1 \mathrm{~Hz}, 3 \mathrm{H}) \mathrm{ppm} .{ }^{13} \mathrm{C}$ NMR (125 MHz, $\mathrm{d}_{6}$-DMSO) $\delta 187.6,167.9,160.4,149.3$, $140.3,137.1,126.8,125.0,123.4,122.3,121.7,120.0,118.0,114.2,111.8,111.1,76.8,76.6,74.7$, 62.8, 51.2, 47.6, 37.3, 26.1, 13.8 ppm. HRMS: [M-H] $]^{-}$calcd. For $\mathrm{C}_{25} \mathrm{H}_{18} \mathrm{FN}_{2} \mathrm{O}_{6}{ }^{-}$461.1154; found: 461.1152. $[\alpha]_{D}^{20}-147\left(c=0.25\right.$ in $\left.\mathrm{CHCl}_{3}\right)$. The enantiomeric excess was determined by HPLC analysis on Daicel Chiralpak IC column [ $n$-hexane $/ i-\mathrm{PrOH}=80 / 20,1 \mathrm{~mL} / \mathrm{min}], \lambda=220 \mathrm{~nm}, t_{\text {minor }}=10.31 \mathrm{~min}$, $t_{\text {major }}=12.08 \mathrm{~min}, \boldsymbol{e e}=\mathbf{9 7 \%}$. 
Ethyl $\quad(5 \mathrm{a} R, 7 R, 7 \mathrm{a} R, 13 \mathrm{a} S, 13 \mathrm{bS})-6,6-$ dicyano-7-hydroxy-3-methoxy-8-oxo-5a,6,7,7a,8,13a,13b,14octahydrochromeno[2,3-a]xanthene-7-carboxylate (4c)

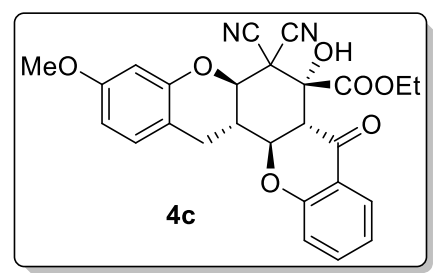

4c was obtained as a white solid ( $40 \mathrm{mg}, 84 \%$ ) by General procedure 9. ${ }^{1} \mathrm{H}$ NMR (500 MHz, $\mathrm{d}_{6}$-DMSO) $\delta 7.93(\mathrm{~s}, 1 \mathrm{H}), 7.78(\mathrm{~d}, J=7.5 \mathrm{~Hz}, 1 \mathrm{H}), 7.66$ $(t, J=7.7 \mathrm{~Hz}, 1 \mathrm{H}), 7.19-7.11(\mathrm{~m}, 3 \mathrm{H}), 6.58(\mathrm{dd}, J=8.4,2.2 \mathrm{~Hz}, 1 \mathrm{H}), 6.53$ $(\mathrm{d}, J=2.1 \mathrm{~Hz}, 1 \mathrm{H}), 4.73(\mathrm{~d}, J=10.9 \mathrm{~Hz}, 1 \mathrm{H}), 4.58(\mathrm{dd}, J=12.8,10.4 \mathrm{~Hz}$, 1H), $4.50-4.26(\mathrm{~m}, 2 \mathrm{H}), 3.83(\mathrm{~d}, J=12.9 \mathrm{~Hz}, 1 \mathrm{H}), 3.74(\mathrm{~s}, 3 \mathrm{H}), 3.27$ (dd, $J=16.0,4.8 \mathrm{~Hz}, 1 \mathrm{H}), 2.96$ $(\mathrm{dd}, J=15.8,11.5 \mathrm{~Hz}, 1 \mathrm{H}), 2.62-2.50(\mathrm{~m}, 1 \mathrm{H}), 1.32(\mathrm{t}, J=7.0 \mathrm{~Hz}, 3 \mathrm{H}) \mathrm{ppm} .{ }^{13} \mathrm{C}$ NMR $(125 \mathrm{MHz}$, $\mathrm{d}_{6}$-DMSO) $\delta 187.7,168.0,160.5,159.0,152.9,137.1,130.2,126.8,122.2,120.0,118.0,112.1,111.3$, 109.2, 100.8, 77.0, 76.6, 74.6, 62.8, 55.3, 51.2, 47.7, 37.8, 25.8, 13.8 ppm. HRMS: [M-H]' calcd. For $\mathrm{C}_{26} \mathrm{H}_{21} \mathrm{~N}_{2} \mathrm{O}_{7}^{-}$473.1354; found: 473.1359. [ $\left.\alpha\right]_{D}{ }^{20}-90$ ( $\mathrm{c}=0.25$ in $\mathrm{CHCl}_{3}$ ). The enantiomeric excess was determined by HPLC analysis on Daicel Chiralpak IC column [ $n$-hexane $/ \mathrm{i}-\mathrm{PrOH}=80 / 20,1 \mathrm{~mL} / \mathrm{min}$ ], $\lambda$ $=220 \mathrm{~nm}, t_{\text {minor }}=10.17 \mathrm{~min}, t_{\text {major }}=11.93 \mathrm{~min}, \boldsymbol{e e}=\mathbf{9 3} \%$.

Ethyl $(5 \mathrm{a}, 7 R, 7 \mathrm{a} R, 13 \mathrm{a} S, 13 \mathrm{bS})-3-c h l o r o-6,6-d i c y a n o-7-h y d r o x y-8-0 x o-5 a, 6,7,7 \mathrm{a}, 8,13 \mathrm{a}, 13 \mathrm{~b}, 14-$ octahydrochromeno[2,3-a]xanthene-7-carboxylate (4d)

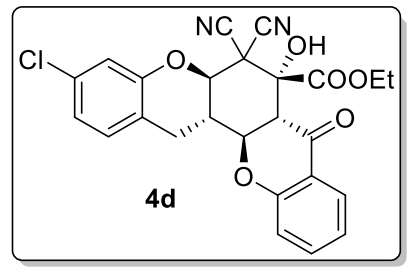

4d was obtained as a white solid (40 $\mathrm{mg}, 84 \%)$ by General procedure 9. ${ }^{1} \mathrm{H}$ NMR (500 MHz, d6-DMSO) $\delta 7.96(\mathrm{~s}, 1 \mathrm{H}), 7.78$ (dd, $\left.J=8.0,1.5 \mathrm{~Hz}, 1 \mathrm{H}\right)$, $7.70-7.63(\mathrm{~m}, 1 \mathrm{H}), 7.30(\mathrm{~d}, J=8.2 \mathrm{~Hz}, 1 \mathrm{H}), 7.19-7.13(\mathrm{~m}, 2 \mathrm{H}), 7.08(\mathrm{~s}$, 1H), 7.05 (dd, $J=8.1,1.8 \mathrm{~Hz}, 1 \mathrm{H}), 4.83(\mathrm{~d}, J=10.9 \mathrm{~Hz}, 1 \mathrm{H}), 4.59$ (dd, $J=$ 12.9, $10.2 \mathrm{~Hz}, 1 \mathrm{H}), 4.48-4.31(\mathrm{~m}, 2 \mathrm{H}), 3.84(\mathrm{~d}, J=12.9 \mathrm{~Hz}, 1 \mathrm{H}), 3.35(\mathrm{dd}, J=11.5,4.8 \mathrm{~Hz}, 1 \mathrm{H}), 3.04$ (dd, $J=16.3,11.5 \mathrm{~Hz}, 1 \mathrm{H}), 2.64-2.54(\mathrm{~m}, 1 \mathrm{H}), 1.32(\mathrm{t}, J=7.1 \mathrm{~Hz}, 3 \mathrm{H})$ ppm. ${ }^{13} \mathrm{C}$ NMR $(125 \mathrm{MHz}$, $\mathrm{d}_{6}$-DMSO) $\delta 187.6,167.9,160.4,152.9,137.1,131.6,131.2,126.8,122.2,121.9,120.0,119.7,117.9$, $116.1,111.9,111.1,76.8,76.6,74.7,62.8,51.2,47.6,37.3,25.9,13.8$ ppm. HRMS: [M-H]' calcd. For $\mathrm{C}_{25} \mathrm{H}_{18} \mathrm{ClN}_{2} \mathrm{O}_{6}{ }^{-}$477.0859; found: 477.0856. [ $\left.\alpha\right]_{D}{ }^{20}-116$ ( $\mathrm{c}=0.25$ in $\mathrm{CHCl}_{3}$ ). The enantiomeric excess was determined by HPLC analysis on Daicel Chiralpak IC column [ $n$-hexane/i-PrOH $=80 / 20$, $1 \mathrm{~mL} / \mathrm{min}], \lambda=220 \mathrm{~nm}, t_{\text {minor }}=8.48 \mathrm{~min}, t_{\text {major }}=10.35 \mathrm{~min}, \boldsymbol{e e}=\mathbf{9 6 \%}$. 


\section{octahydrochromeno[2,3-a]xanthene-7-carboxylate (4e)}

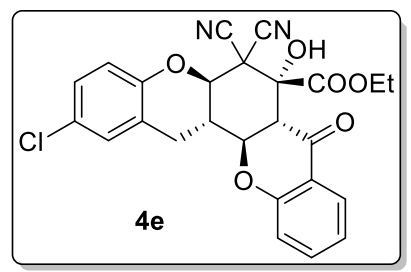

4e was obtained as a white solid ( $39 \mathrm{mg}, 82 \%$ ) by General procedure $9 .{ }^{1} \mathbf{H}$ NMR $\left(500 \mathrm{MHz}, \mathrm{d}_{6}-\mathrm{DMSO}\right) \delta 7.96(\mathrm{~s}, 1 \mathrm{H}), 7.80-7.75(\mathrm{~m}, 1 \mathrm{H}), 7.69-7.63$ $(\mathrm{m}, 1 \mathrm{H}), 7.36(\mathrm{~d}, J=1.7 \mathrm{~Hz}, 1 \mathrm{H}), 7.21(\mathrm{dd}, J=8.7,2.2 \mathrm{~Hz}, 1 \mathrm{H}), 7.15(\mathrm{t}, J=$ $8.3 \mathrm{~Hz}, 2 \mathrm{H}), 7.00(\mathrm{~d}, J=8.7 \mathrm{~Hz}, 1 \mathrm{H}), 4.80(\mathrm{~d}, J=10.9 \mathrm{~Hz}, 1 \mathrm{H}), 4.59$ (dd, $J=$ 12.8, $10.3 \mathrm{~Hz}, 1 \mathrm{H}), 4.49-4.31(\mathrm{~m}, 2 \mathrm{H}), 3.84(\mathrm{~d}, J=12.9 \mathrm{~Hz}, 1 \mathrm{H}), 3.41-3.33(\mathrm{~m}, 1 \mathrm{H}), 3.07(\mathrm{dd}, J=$ 16.4, $11.5 \mathrm{~Hz}, 1 \mathrm{H}), 2.66-2.54(\mathrm{~m}, 1 \mathrm{H}), 1.32(\mathrm{t}, J=7.1 \mathrm{~Hz}, 3 \mathrm{H}) \mathrm{ppm} .{ }^{13} \mathrm{C}$ NMR $\left(125 \mathrm{MHz}, \mathrm{d}_{6}-\mathrm{DMSO}\right) \delta$ $187.6,167.9,160.4,151.1,137.1,129.2,127.6,126.8,125.5,122.8,122.2,120.0,118.0,117.9$, 111.9, 111.2, 76.9, 76.6, 74.6, 62.8, 51.2, 47.6, 37.2, 26.2, 13.8 ppm. HRMS: [M-H]' calcd. For $\mathrm{C}_{25} \mathrm{H}_{18} \mathrm{ClN}_{2} \mathrm{O}_{6}{ }^{-}$477.0859; found: 477.0861. [ $\left.\alpha\right]_{D}{ }^{20}-120$ ( $\mathrm{c}=0.25$ in $\mathrm{CHCl}_{3}$ ). The enantiomeric excess was determined by HPLC analysis on Daicel Chiralpak IC column [ $n$-hexane/i-PrOH $=80 / 20$, $1 \mathrm{~mL} / \mathrm{min}], \lambda=220 \mathrm{~nm}, t_{\text {minor }}=8.76 \mathrm{~min}, t_{\text {maJor }}=9.91 \mathrm{~min}, \boldsymbol{e} \boldsymbol{e}=\mathbf{9 2} \%$.

Ethyl $\quad(5 \mathrm{a} R, 7 R, 7 \mathrm{a} R, 13 \mathrm{a} S, 13 \mathrm{bS})-6,6-d i c y a n o-7-h y d r o x y-12-m e t h y l-8-0 x o-5 a, 6,7,7 a, 8,13 a, 13 b, 14-$ octahydrochromeno[2,3-a]xanthene-7-carboxylate (4f)

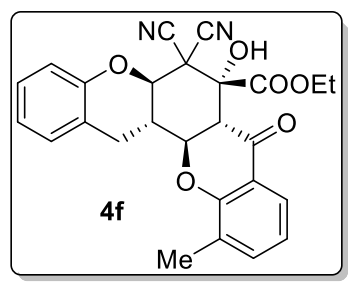

4f was obtained as a white solid (40 mg, 87\%) by General procedure $9 .{ }^{1} \mathbf{H}$ NMR $\left(500 \mathrm{MHz}, \mathrm{d}_{6}-\mathrm{DMSO}\right) \delta 7.91(\mathrm{~s}, 1 \mathrm{H}), 7.62(\mathrm{~d}, J=7.9 \mathrm{~Hz}, 1 \mathrm{H}), 7.54(\mathrm{~d}, J=$ $7.2 \mathrm{~Hz}, 1 \mathrm{H}), 7.27(\mathrm{~d}, J=7.5 \mathrm{~Hz}, 1 \mathrm{H}), 7.20(\mathrm{t}, J=7.5 \mathrm{~Hz}, 1 \mathrm{H}), 7.05(\mathrm{t}, J=7.6 \mathrm{~Hz}$ 1H), $7.02-6.95(\mathrm{~m}, 2 \mathrm{H}), 4.77(\mathrm{~d}, J=10.9 \mathrm{~Hz}, 1 \mathrm{H}), 4.55(\mathrm{dd}, J=12.9,10.2 \mathrm{~Hz}$, $1 \mathrm{H}), 4.47-4.30(\mathrm{~m}, 2 \mathrm{H}), 3.79(\mathrm{~d}, J=13.0 \mathrm{~Hz}, 1 \mathrm{H}), 3.37(\mathrm{dd}, J=16.7,5.1 \mathrm{~Hz}$ 1H), $3.10(\mathrm{dd}, J=16.3,11.5 \mathrm{~Hz}, 1 \mathrm{H}), 2.67-2.55(\mathrm{~m}, 1 \mathrm{H}), 2.30(\mathrm{~s}, 3 \mathrm{H}), 1.32(\mathrm{t}, J=7.1 \mathrm{~Hz}, 3 \mathrm{H}) \mathrm{ppm} .{ }^{13} \mathrm{C}$ NMR $\left(125 \mathrm{MHz}, \mathrm{d}_{6}\right.$-DMSO) $\delta 187.9,168.0,158.6,152.3,137.5,129.8,127.8,127.0,124.3,121.9$, 121.6, 120.5, 119.7, 116.2, 112.0, 111.3, 76.9, 76.6, 74.5, 62.8, 51.1, 47.7, 37.7, 26.3, 15.0, 13.8 ppm. HRMS: [M-H] ${ }^{-}$calcd. For $\mathrm{C}_{26} \mathrm{H}_{21} \mathrm{~N}_{2} \mathrm{O}_{6}{ }^{-} 457.1405$; found: 457.1409. [ $\left.\alpha\right]_{D^{20}}-73$ (c $=0.25$ in $\mathrm{CHCl}_{3}$ ). The enantiomeric excess was determined by HPLC analysis on Daicel Chiralpak IC column [n-hexane $/ i-\mathrm{PrOH}=80 / 20,1 \mathrm{~mL} / \mathrm{min}], \lambda=220 \mathrm{~nm}, t_{\text {minor }}=8.27 \mathrm{~min}, t_{\text {major }}=10.33 \mathrm{~min}, \boldsymbol{e e}=\mathbf{9 9 . 5 \%}$. 
Ethyl $\quad(5 a R, 7 R, 7 a R, 13 a S, 13 b S)-12-c h l o r o-6,6-d i c y a n o-7-h y d r o x y-8-o x o-5 a, 6,7,7 a, 8,13 a, 13 b, 14-$ octahydrochromeno[2,3-a]xanthene-7-carboxylate $(4 \mathrm{~g})$

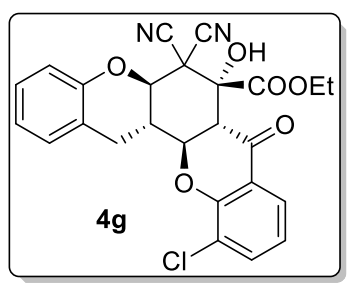

$\mathbf{4 g}$ was obtained as a white solid (39 $\mathrm{mg}, 82 \%$ ) by General procedure $\mathbf{9}^{{ }^{1} \mathbf{H}}$ NMR (500 MHz, d $\left.\mathrm{d}_{6}-\mathrm{DMSO}\right) \delta 7.98(\mathrm{~s}, 1 \mathrm{H}), 7.84(\mathrm{dd}, J=7.8,1.4 \mathrm{~Hz}, 1 \mathrm{H}), 7.75$ (dd, $J=7.9,1.4 \mathrm{~Hz}, 1 \mathrm{H}$ ), 7.27 (d, J=7.5 Hz, 1H), 7.20 (t, J= $7.7 \mathrm{~Hz}, 1 \mathrm{H}), 7.16(\mathrm{t}$, $J=7.9 \mathrm{~Hz}, 1 \mathrm{H}), 7.02-6.96(\mathrm{~m}, 2 \mathrm{H}), 4.78(\mathrm{~d}, J=10.9 \mathrm{~Hz}, 1 \mathrm{H}), 4.69(\mathrm{dd}, J=$ 12.9, $10.2 \mathrm{~Hz}, 1 \mathrm{H}), 4.49-4.29(\mathrm{~m}, 2 \mathrm{H}), 4.02(\mathrm{~d}, J=13.0 \mathrm{~Hz}, 1 \mathrm{H}), 3.33(\mathrm{dd}, J=$ 16.6, $4.9 \mathrm{~Hz}, 1 \mathrm{H}), 3.14(\mathrm{dd}, J=16.3,11.5 \mathrm{~Hz}, 1 \mathrm{H}), 2.71-2.61(\mathrm{~m}, 1 \mathrm{H}), 1.32(\mathrm{t}, J=7.1 \mathrm{~Hz}, 3 \mathrm{H}) \mathrm{ppm}$. ${ }^{13} \mathrm{C}$ NMR $\left(125 \mathrm{MHz}, \mathrm{d}_{6}\right.$-DMSO) $\delta 187.3,167.8,155.8,152.2,136.6,129.8,127.9,125.7,122.6,122.0$, $121.8,121.6,120.3,116.2,112.0,111.2,77.7,76.6,74.4,62.9,50.9,47.7,37.6,26.3,13.8$ ppm.

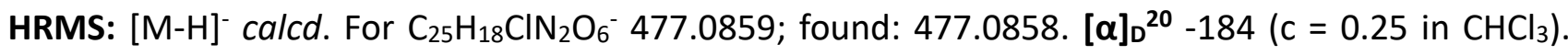
The enantiomeric excess was determined by HPLC analysis on Daicel Chiralpak IC column $[n$-hexane $/ i-\mathrm{PrOH}=80 / 20,1 \mathrm{~mL} / \mathrm{min}], \lambda=220 \mathrm{~nm}, t_{\text {minor }}=7.15 \mathrm{~min}, t_{\text {major }}=9.17 \mathrm{~min}$, ee $=\mathbf{9 9 . 5 \%}$.

Ethyl $\quad(5 \mathrm{a} R, 7 R, 7 \mathrm{a} R, 13 \mathrm{aS}, 13 \mathrm{bS})-12-\mathrm{bromo}-6,6-d i c y a n o-7-h y d r o x y-8-0 x 0-5 \mathrm{a}, 6,7,7 \mathrm{a}, 8,13 \mathrm{a}, 13 \mathrm{~b}, 14-$ octahydrochromeno[2,3-a]xanthene-7-carboxylate (4h)

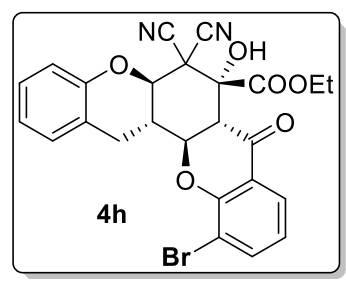

4h was obtained as a white solid (44 $\mathrm{mg}, 84 \%$ ) by General procedure $\mathbf{9}^{{ }^{1} \mathbf{H}}$ NMR (500 MHz, d6-DMSO) $\delta 8.00-7.95(\mathrm{~m}, 2 \mathrm{H}), 7.79(\mathrm{dd}, J=7.8,1.3 \mathrm{~Hz}, 1 \mathrm{H})$, $7.25(\mathrm{~d}, J=7.5 \mathrm{~Hz}, 1 \mathrm{H}), 7.20(\mathrm{t}, J=7.7 \mathrm{~Hz}, 1 \mathrm{H}), 7.10(\mathrm{t}, J=7.8 \mathrm{~Hz}, 1 \mathrm{H}), 7.03-$ $6.96(\mathrm{~m}, 2 \mathrm{H}), 4.78(\mathrm{~d}, J=10.9 \mathrm{~Hz}, 1 \mathrm{H}), 4.68(\mathrm{dd}, J=12.9,10.3 \mathrm{~Hz}, 1 \mathrm{H}), 4.48-$ $4.30(\mathrm{~m}, 2 \mathrm{H}), 4.01(\mathrm{~d}, J=13.0 \mathrm{~Hz}, 1 \mathrm{H}), 3.34(\mathrm{dd}, J=16.4,5.0 \mathrm{~Hz}, 1 \mathrm{H}), 3.15(\mathrm{dd}, J=16.3,11.5 \mathrm{~Hz}, 1 \mathrm{H})$, $2.72-2.60(\mathrm{~m}, 1 \mathrm{H}), 1.32(\mathrm{t}, J=7.1 \mathrm{~Hz}, 3 \mathrm{H}) \mathrm{ppm} .{ }^{13} \mathrm{C}$ NMR $\left(125 \mathrm{MHz}, \mathrm{d}_{6}\right.$-DMSO) $\delta 187.3,167.8,156.7$, $152.3,139.7,129.7,127.9,126.4,123.2,122.0,121.5,120.3,116.3,112.0,111.1,109.5,77.6,76.6$, 74.4, 62.9, 50.8, 47.7, 37.6, 26.4, 13.8 ppm. HRMS: [M-H] $]^{-}$calcd. For $\mathrm{C}_{25} \mathrm{H}_{18} \mathrm{BrN}_{2} \mathrm{O}_{6}{ }^{-}$521.0354; found: 521.0354. $[\alpha]_{D}{ }^{20}-128\left(c=0.25\right.$ in $\mathrm{CHCl}_{3}$ ). The enantiomeric excess was determined by HPLC analysis on Daicel Chiralpak IC column [ $n$-hexane/i-PrOH $=80 / 20,1 \mathrm{~mL} / \mathrm{min}$ ], $\lambda=220 \mathrm{~nm}, t_{\text {minor }}=7.31 \mathrm{~min}$, $t_{\text {major }}=9.51 \mathrm{~min}, \boldsymbol{e e}=\mathbf{9 9 . 8 \%}$. 
Ethyl $\quad(5 \mathrm{a} R, 7 R, 7 \mathrm{a} R, 13 \mathrm{aS}, 13 \mathrm{bS})-6,6$-dicyano-7-hydroxy-11-methoxy-8-oxo-5a,6,7,7a,8,13a,13b,14octahydrochromeno[2,3-a]xanthene-7-carboxylate (4i)

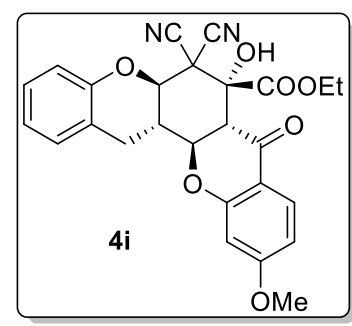

4i was obtained as a white solid (40 mg, $84 \%$ ) by General procedure ${ }^{9} .{ }^{1} \mathbf{H}$ $\operatorname{NMR}\left(500 \mathrm{MHz}, \mathrm{d}_{6}-\mathrm{DMSO}\right) \delta 7.86(\mathrm{~s}, 1 \mathrm{H}), 7.71(\mathrm{~d}, J=8.8 \mathrm{~Hz}, 1 \mathrm{H}), 7.24(\mathrm{~d}, J=$ $7.5 \mathrm{~Hz}, 1 \mathrm{H}), 7.19(\mathrm{t}, J=7.5 \mathrm{~Hz}, 1 \mathrm{H}), 7.03-6.93(\mathrm{~m}, 2 \mathrm{H}), 6.72(\mathrm{dd}, J=8.8,2.3$ $\mathrm{Hz}, 1 \mathrm{H}), 6.68(\mathrm{~d}, J=2.2 \mathrm{~Hz}, 1 \mathrm{H}), 4.76(\mathrm{~d}, J=10.9 \mathrm{~Hz}, 1 \mathrm{H}), 4.56(\mathrm{dd}, J=12.7$, $10.3 \mathrm{~Hz}, 1 \mathrm{H}), 4.47-4.31(\mathrm{~m}, 2 \mathrm{H}), 3.86(\mathrm{~s}, 3 \mathrm{H}), 3.71(\mathrm{~d}, J=12.8 \mathrm{~Hz}, 1 \mathrm{H}), 3.33$ (dd, $J=16.4,4.9 \mathrm{~Hz}, 1 \mathrm{H}), 3.08(\mathrm{dd}, J=16.2,11.5 \mathrm{~Hz}, 1 \mathrm{H}), 2.63-2.53(\mathrm{~m}, 1 \mathrm{H}), 1.32(\mathrm{t}, J=7.1 \mathrm{~Hz}, 3 \mathrm{H})$ ppm. ${ }^{13} \mathrm{C}$ NMR (125 MHz, d6-DMSO) $\delta$ 186.0, 168.0, 166.2, 162.6, 152.3, 129.7, 128.5, 127.8, 122.0, 120.5, 116.2, 113.7, 112.1, 111.3, 111.0, 100.9, 77.3, 76.6, 74.5, 62.7, 56.1, 50.8, 47.7, 37.6, 26.4, 13.8 ppm. HRMS: [M-H] $]^{-}$calcd. For $\mathrm{C}_{26} \mathrm{H}_{21} \mathrm{~N}_{2} \mathrm{O}_{7}^{-}$473.1354; found: 473.1348 . [ $\left.\alpha\right]_{D^{20}}-119$ (c = 0.25 in $\mathrm{CHCl}_{3}$ ). The enantiomeric excess was determined by HPLC analysis on Daicel Chiralpak IC column $[n$-hexane $/ i$-PrOH $=80 / 20,1 \mathrm{~mL} / \mathrm{min}], \lambda=220 \mathrm{~nm}, t_{\text {minor }}=12.25 \mathrm{~min}, t_{\text {major }}=14.97 \mathrm{~min}, \boldsymbol{e e}=\mathbf{9 2 \%}$.

Ethyl $\quad(5 \mathrm{a} R, 7 R, 7 \mathrm{a} R, 13 \mathrm{a} S, 13 \mathrm{bS})-11-\mathrm{bromo}-6,6-d i c y a n o-7-h y d r o x y-8-0 x 0-5 \mathrm{a}, 6,7,7 \mathrm{a}, 8,13 \mathrm{a}, 13 \mathrm{~b}, 14-$ octahydrochromeno[2,3-a]xanthene-7-carboxylate (4j)

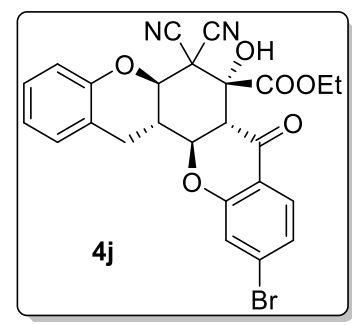

4j was obtained as a white solid (46 mg, $88 \%$ ) by General procedure ${ }^{9} .{ }^{1} \mathbf{H}$ NMR (500 MHz, d6-DMSO) $\delta 7.95(\mathrm{~s}, 1 \mathrm{H}), 7.70(\mathrm{~d}, J=8.4 \mathrm{~Hz}, 1 \mathrm{H}), 7.47$ (d, J = $1.5 \mathrm{~Hz}, 1 \mathrm{H}), 7.35$ (dd, $J=8.4,1.7 \mathrm{~Hz}, 1 \mathrm{H}), 7.23(\mathrm{~d}, J=7.5 \mathrm{~Hz}, 1 \mathrm{H}), 7.19(\mathrm{t}, J=$ $7.6 \mathrm{~Hz}, 1 \mathrm{H}), 7.03-6.95(\mathrm{~m}, 2 \mathrm{H}), 4.76(\mathrm{~d}, J=10.9 \mathrm{~Hz}, 1 \mathrm{H}), 4.63(\mathrm{dd}, J=12.9$, $10.2 \mathrm{~Hz}, 1 \mathrm{H}), 4.48-4.29(\mathrm{~m}, 2 \mathrm{H}), 3.91(\mathrm{~d}, J=13.0 \mathrm{~Hz}, 1 \mathrm{H}), 3.34-3.29(\mathrm{~m}, 1 \mathrm{H})$, $3.07(\mathrm{dd}, J=16.3,11.5 \mathrm{~Hz}, 1 \mathrm{H}), 2.64-2.54(\mathrm{~m}, 1 \mathrm{H}), 1.32(\mathrm{t}, J=7.1 \mathrm{~Hz}, 3 \mathrm{H}) \mathrm{ppm} .{ }^{13} \mathrm{C}$ NMR $(125 \mathrm{MHz}$, $\mathrm{d}_{6}$-DMSO) $\delta 187.1,167.9,160.8,152.2,130.2,129.7,128.5,127.9,125.5,122.0,120.9,120.4,119.3$, $116.2,112.0,111.2,77.4,76.5,74.4,62.8,51.0,47.7,37.5,26.3,13.8$ ppm. HRMS: [M-H]' calcd. For $\mathrm{C}_{25} \mathrm{H}_{18} \mathrm{BrN}_{2} \mathrm{O}_{6}{ }^{-}$521.0354; found: 521.0357. [ $\left.\alpha\right]_{D^{2}}{ }^{20}-122$ ( $\mathrm{c}=0.25$ in $\mathrm{CHCl}_{3}$ ). The enantiomeric excess was determined by HPLC analysis on Daicel Chiralpak IC column [ $n$-hexane/i-PrOH $=80 / 20$, $1 \mathrm{~mL} / \mathrm{min}], \lambda=220 \mathrm{~nm}, t_{\text {minor }}=7.95 \mathrm{~min}, t_{\text {major }}=10.44 \mathrm{~min}, \boldsymbol{e} \boldsymbol{e}=\mathbf{9 4 \%}$. 
Ethyl $\quad(5 a R, 7 R, 7 a R, 13 a S, 13 b S)-6,6-d i c y a n o-7-h y d r o x y-10-m e t h y l-8-0 x o-5 a, 6,7,7 a, 8,13 a, 13 b, 14-$ octahydrochromeno[2,3-a]xanthene-7-carboxylate (4k)

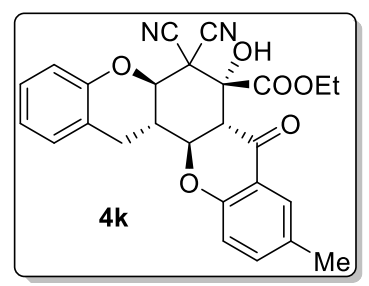

$\mathbf{4 k}$ was obtained as a white solid (40 mg, $87 \%$ ) by General procedure ${ }^{9} .{ }^{1} \mathbf{H}$ NMR (500 MHz, d $\mathrm{d}_{6}$-DMSO) $\delta 7.90(\mathrm{~s}, 1 \mathrm{H}), 7.57(\mathrm{~s}, 1 \mathrm{H}), 7.48(\mathrm{dd}, J=8.5,2.0$ $\mathrm{Hz}, 1 \mathrm{H}), 7.25$ (d, J= $7.5 \mathrm{~Hz}, 1 \mathrm{H}), 7.19$ (t, J= 7.6 Hz, 1H), 7.07 (d, J=8.4 Hz, 1H), $7.02-6.94(\mathrm{~m}, 2 \mathrm{H}), 4.75(\mathrm{~d}, J=10.9 \mathrm{~Hz}, 1 \mathrm{H}), 4.54(\mathrm{dd}, J=12.9,10.2 \mathrm{~Hz}, 1 \mathrm{H})$, $4.48-4.30(\mathrm{~m}, 2 \mathrm{H}), 3.78(\mathrm{~d}, J=12.9 \mathrm{~Hz}, 1 \mathrm{H}), 3.33(\mathrm{dd}, J=16.4,4.9 \mathrm{~Hz}, 1 \mathrm{H}), 3.06(\mathrm{dd}, J=16.2,11.5$ $\mathrm{Hz}, 1 \mathrm{H}), 2.64-2.54(\mathrm{~m}, 1 \mathrm{H}), 2.29(\mathrm{~s}, 3 \mathrm{H}), 1.32(\mathrm{t}, \mathrm{J}=7.1 \mathrm{~Hz}, 3 \mathrm{H})$ ppm. ${ }^{13} \mathrm{C}$ NMR $\left(125 \mathrm{MHz}, \mathrm{d}_{6}-\mathrm{DMSO}\right)$ $\delta 187.7,168.0,158.5,152.2,138.0,131.3,129.7,127.8,126.2,121.9,120.5,119.6,117.8,116.2$, 112.0, 111.3, 77.0, 76.5, 74.5, 62.8, 51.2, 47.7, 37.6, 26.4, 19.9, 13.8 ppm. HRMS: [M-H]' calcd. For $\mathrm{C}_{26} \mathrm{H}_{21} \mathrm{~N}_{2} \mathrm{O}_{6}-457.1405$; found: 457.1405. [ $\left.\alpha\right]_{D}{ }^{20}-189$ ( $\mathrm{c}=0.25$ in $\mathrm{CHCl}_{3}$ ). The enantiomeric excess was determined by HPLC analysis on Daicel Chiralpak IC column [ $n$-hexane/i-PrOH $=80 / 20,1 \mathrm{~mL} / \mathrm{min}], \lambda$ $=220 \mathrm{~nm}, t_{\text {minor }}=8.27 \mathrm{~min}, t_{\text {major }}=10.32 \mathrm{~min}, \boldsymbol{e e}=\mathbf{9 2} \%$.

Ethyl $\quad(5 a R, 7 R, 7 a R, 13 a S, 13 b S)-6,6-d i c y a n o-10-f l u o r o-7-h y d r o x y-8-o x o-5 a, 6,7,7 a, 8,13 a, 13 b, 14-$ octahydrochromeno[2,3-a]xanthene-7-carboxylate (4I)

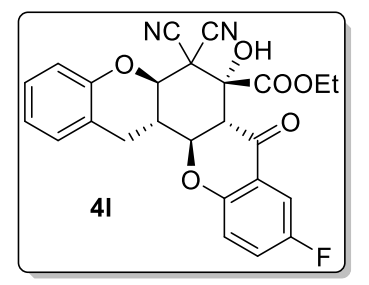

4 l was obtained as a white solid (34 mg, 74\%) by General procedure ${ }^{9} .{ }^{1} \mathbf{H}$ NMR (500 MHz, d6-DMSO) $\delta 7.97$ (s, 1H), $7.59-7.53(\mathrm{~m}, 1 \mathrm{H}), 7.50$ (dd, J = 8.2, $3.1 \mathrm{~Hz}, 1 \mathrm{H}), 7.27-7.22(\mathrm{~m}, 2 \mathrm{H}), 7.19(\mathrm{t}, J=7.5 \mathrm{~Hz}, 1 \mathrm{H}), 7.02-6.94(\mathrm{~m}, 2 \mathrm{H})$, $4.76(\mathrm{~d}, J=10.9 \mathrm{~Hz}, 1 \mathrm{H}), 4.60(\mathrm{dd}, J=12.9,10.2 \mathrm{~Hz}, 1 \mathrm{H}), 4.49-4.30(\mathrm{~m}, 2 \mathrm{H})$, $3.89(\mathrm{~d}, J=13.0 \mathrm{~Hz}, 1 \mathrm{H}), 3.33(\mathrm{dd}, J=15.6,5.7 \mathrm{~Hz}, 1 \mathrm{H}), 3.07(\mathrm{dd}, J=16.3,11.5 \mathrm{~Hz}, 1 \mathrm{H}), 2.65-2.55$ (m, 1H), 1.32 (t, $J=7.1 \mathrm{~Hz}, 3 \mathrm{H}$ ) ppm. ${ }^{13} \mathrm{C}$ NMR (125 MHz, $\mathrm{d}_{6}$-DMSO) $\delta 187.2,167.9,157.0,156.8$, 152.2 , 129.7, 127.8, 124.4, 122.0, 120.6, 120.4, 120.3, 116.2, 112.0, 111.5, 111.2, 77.3, 76.5, 74.5, 62.9, 51.0, 47.7, 37.5, 26.4, 13.8 ppm. HRMS: [M-H] calcd. For $\mathrm{C}_{25} \mathrm{H}_{18} \mathrm{FN}_{2} \mathrm{O}_{6}{ }^{-}$461.1154; found: 461.1156. $[\alpha]_{D}^{20}-78\left(c=0.25\right.$ in $\mathrm{CHCl}_{3}$ ). The enantiomeric excess was determined by HPLC analysis on Daicel Chiralpak IC column [ $n$-hexane/ $i$-PrOH $=80 / 20,1 \mathrm{~mL} / \mathrm{min}$ ], $\lambda=210 \mathrm{~nm}, t_{\text {minor }}=7.24 \mathrm{~min}$, $t_{\text {major }}=10.52 \mathrm{~min}, \boldsymbol{e e}=\mathbf{9 5 \%}$. 
Ethyl $\quad(5 a R, 7 R, 7 a R, 13 a S, 13 b S)-10$-acetoxy-6,6-dicyano-7-hydroxy-8-oxo-5a,6,7,7a,8,13a,13b,14octahydrochromeno[2,3-a]xanthene-7-carboxylate $(4 \mathrm{~m})$

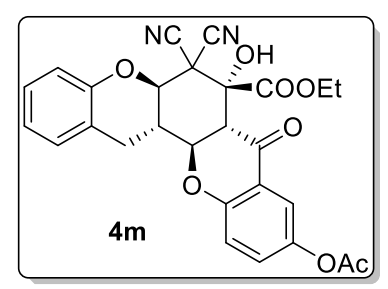

$4 \mathrm{~m}$ was obtained as a white solid (43 $\mathrm{mg}, 86 \%$ ) by General procedure $9 .{ }^{1} \mathbf{H}$ NMR (500 MHz, d $\mathrm{d}_{6}$-DMSO) $\delta 7.94(\mathrm{~s}, 1 \mathrm{H}), 7.49(\mathrm{~s}, 1 \mathrm{H}), 7.46-7.41(\mathrm{~m}, 1 \mathrm{H})$, $7.29-7.15(\mathrm{~m}, 3 \mathrm{H}), 7.03-6.95(\mathrm{~m}, 2 \mathrm{H}), 4.76(\mathrm{~d}, J=10.9 \mathrm{~Hz}, 1 \mathrm{H}), 4.66-$ $4.59(\mathrm{~m}, 1 \mathrm{H}), 4.47-4.31(\mathrm{~m}, 2 \mathrm{H}), 3.91(\mathrm{~d}, J=13.0 \mathrm{~Hz}, 1 \mathrm{H}), 3.37-3.31(\mathrm{~m}$, 1H), 3.08 (dd, $J=16.1,11.7 \mathrm{~Hz}, 1 \mathrm{H}$ ), 2.61 (ddd, $J=16.4,10.8,5.4 \mathrm{~Hz}, 1 \mathrm{H}$ ), $2.27(\mathrm{~s}, 3 \mathrm{H}), 1.32$ (t, $J=7.1$ $\mathrm{Hz}, 3 \mathrm{H}) \mathrm{ppm} .{ }^{13} \mathrm{C}$ NMR (125 MHz, d6-DMSO) $\delta 187.4,169.3,167.9,158.1,152.2,144.8,130.9,129.7$, $127.8,122.0,120.4,120.2,119.2,118.9,116.2,112.0,111.2,77.2,76.5,74.5,62.8,51.1,47.7,37.5$,

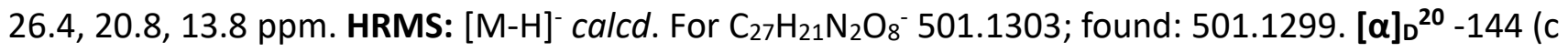
$=0.25$ in $\mathrm{CHCl}_{3}$ ). The enantiomeric excess was determined by HPLC analysis on Daicel Chiralpak IC column $[n$-hexane $/ i-\operatorname{PrOH}=80 / 20,1 \mathrm{~mL} / \mathrm{min}], \lambda=210 \mathrm{~nm}, t_{\text {minor }}=19.65 \mathrm{~min}, t_{\text {major }}=28.51 \mathrm{~min}, \boldsymbol{e e}=$ $90 \%$.

Ethyl $\quad(5 \mathrm{a} R, 7 R, 7 \mathrm{a} R, 13 \mathrm{a} S, 13 \mathrm{bS})-6,6-$ dicyano-7-hydroxy-9-methoxy-8-oxo-5a,6,7,7a,8,13a,13b,14octahydrochromeno[2,3-a]xanthene-7-carboxylate (4n)

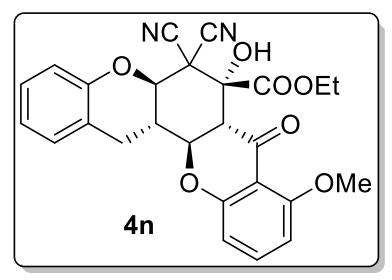

4n was obtained as a white solid (45 mg, 95\%) by General procedure $9 .{ }^{1} \mathbf{H}$ NMR (500 MHz, d 6 -DMSO) $\delta 7.82(s, 1 H), 7.55(t, J=8.4 \mathrm{~Hz}, 1 \mathrm{H}), 7.24(\mathrm{~d}, J=$ $7.3 \mathrm{~Hz}, 1 \mathrm{H}), 7.18(\mathrm{t}, J=7.6 \mathrm{~Hz}, 1 \mathrm{H}), 7.02-6.93(\mathrm{~m}, 2 \mathrm{H}), 6.74(\mathrm{~d}, J=8.4 \mathrm{~Hz}$, 1H), $6.70(\mathrm{~d}, J=8.3 \mathrm{~Hz}, 1 \mathrm{H}), 4.73(\mathrm{~d}, J=10.9 \mathrm{~Hz}, 1 \mathrm{H}), 4.55-4.28(\mathrm{~m}, 3 \mathrm{H})$, $3.82(\mathrm{~s}, 3 \mathrm{H}), 3.64(\mathrm{~d}, J=12.9 \mathrm{~Hz}, 1 \mathrm{H}), 3.30(\mathrm{dd}, J=16.4,4.8 \mathrm{~Hz}, 1 \mathrm{H}), 3.05$ (dd, $J=16.0,11.6 \mathrm{~Hz}, 1 \mathrm{H}$ ), $2.59-2.50(\mathrm{~m}, 1 \mathrm{H}), 1.32(\mathrm{t}, J=7.1 \mathrm{~Hz}, 3 \mathrm{H}) \mathrm{ppm} .{ }^{13} \mathrm{C}$ NMR (125 MHz, d6-DMSO) $\delta$ 185.1, 168.1, 161.8, $160.7,152.3,137.1,129.7,127.8,121.9,120.5,116.2,112.1,111.3,109.9,109.4,105.1,76.6,76.2$, 74.4, 62.7, 56.0, 52.0, 47.8, 37.5, 26.3, 13.9 ppm. HRMS: [M-H] calcd. For $\mathrm{C}_{26} \mathrm{H}_{21} \mathrm{~N}_{2} \mathrm{O}_{7}^{-} 473.1354$; found: 473.1353. [ $\alpha]_{D}{ }^{20}-191\left(c=0.25\right.$ in $\mathrm{CHCl}_{3}$ ). The enantiomeric excess was determined by HPLC analysis on Daicel Chiralpak IC column [ $n$-hexane/i-PrOH $=80 / 20,1 \mathrm{~mL} / \mathrm{min}$ ], $\lambda=210 \mathrm{~nm}, t_{\text {major }}=$ $14.92 \mathrm{~min}, t_{\text {minor }}=20.35 \mathrm{~min}, \boldsymbol{e e}=\mathbf{9 2} \%$. 


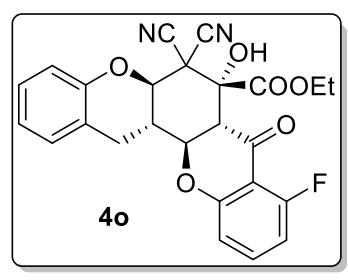

40 was obtained as a white solid (38 $\mathrm{mg}, 82 \%$ ) by General procedure $9 .{ }^{1} \mathbf{H}$ NMR (500 MHz, $d_{6}$-DMSO) $\delta 7.95(\mathrm{~s}, 1 \mathrm{H}), 7.66(\mathrm{dd}, J=14.3,8.3 \mathrm{~Hz}, 1 \mathrm{H}), 7.25$ $(\mathrm{d}, J=7.4 \mathrm{~Hz}, 1 \mathrm{H}), 7.19(\mathrm{t}, J=7.5 \mathrm{~Hz}, 1 \mathrm{H}), 7.04-6.90(\mathrm{~m}, 4 \mathrm{H}), 4.75(\mathrm{~d}, J=10.9$ $\mathrm{Hz}, 1 \mathrm{H}), 4.62(\mathrm{dd}, J=12.8,10.3 \mathrm{~Hz}, 1 \mathrm{H}), 4.48-4.32(\mathrm{~m}, 2 \mathrm{H}), 3.88(\mathrm{~d}, J=12.9$ $\mathrm{Hz}, 1 \mathrm{H}), 3.35-3.27(\mathrm{~m}, 1 \mathrm{H}), 3.07(\mathrm{dd}, J=16.2,11.5 \mathrm{~Hz}, 1 \mathrm{H}), 2.62-2.52(\mathrm{~m}, 1 \mathrm{H}), 1.32(\mathrm{t}, J=7.1 \mathrm{~Hz}$, 3H) ppm. ${ }^{13} \mathrm{C}$ NMR (125 MHz, d $\mathrm{d}_{6}$-DMSO) $\delta 185.4,167.9,161.2,161.0,152.2,137.5,129.7,127.9$, 122.0, 120.4, 116.2, 114.0, 112.0, 111.2, 110.0, 109.4, 76.8, 76.5, 74.4, 62.8, 51.6, 47.7, 37.5, 26.3, 13.8 ppm. HRMS: [M-H] $]^{-}$calcd. For $\mathrm{C}_{25} \mathrm{H}_{18} \mathrm{FN}_{2} \mathrm{O}_{6}{ }^{-} 461.1154$; found: 461.1150 . [ $\left.\alpha\right]_{\mathrm{D}}{ }^{20}-166$ (c=0.25 in $\mathrm{CHCl}_{3}$ ). The enantiomeric excess was determined by HPLC analysis on Daicel Chiralpak IA column $[n$-hexane $/ i-\operatorname{PrOH}=85 / 15,1 \mathrm{~mL} / \mathrm{min}], \lambda=210 \mathrm{~nm}, t_{\text {minor }}=18.34 \mathrm{~min}, t_{\text {major }}=20.32 \mathrm{~min}, \boldsymbol{e e}=\mathbf{9 2} \%$.

Ethyl $(5 a R, 7 R, 7 a R, 13 a S, 13 b S)-9-c h l o r o-6,6-d i c y a n o-7-h y d r o x y-8-o x o-5 a, 6,7,7 a, 8,13 a, 13 b, 14-$ octahydrochromeno[2,3-a]xanthene-7-carboxylate (4p)

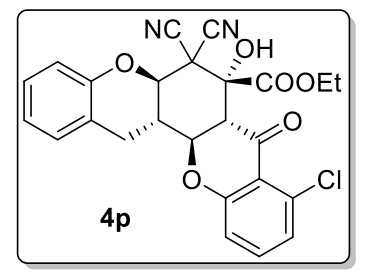

$4 p$ was obtained as a white solid (38 $\mathrm{mg}, 79 \%$ ) by General procedure $9 .{ }^{1} \mathbf{H}$ NMR $\left(500 \mathrm{MHz}, \mathrm{d}_{6}-\mathrm{DMSO}\right) \delta 7.94(\mathrm{~s}, 1 \mathrm{H}), 7.59(\mathrm{t}, J=8.2 \mathrm{~Hz}, 1 \mathrm{H}), 7.25(\mathrm{~d}, J=$ $7.5 \mathrm{~Hz}, 1 \mathrm{H}), 7.22-7.14(\mathrm{~m}, 3 \mathrm{H}), 7.03-6.94(\mathrm{~m}, 2 \mathrm{H}), 4.75(\mathrm{~d}, J=10.9 \mathrm{~Hz}, 1 \mathrm{H})$, $4.62(\mathrm{dd}, J=12.9,10.2 \mathrm{~Hz}, 1 \mathrm{H}), 4.46-4.33(\mathrm{~m}, 2 \mathrm{H}), 3.90(\mathrm{~d}, J=13.0 \mathrm{~Hz}, 1 \mathrm{H})$, $3.33(\mathrm{dd}, J=16.4,4.8 \mathrm{~Hz}, 1 \mathrm{H}), 3.07(\mathrm{dd}, J=16.1,11.6 \mathrm{~Hz}, 1 \mathrm{H}), 2.56(\mathrm{ddd}, J=21.8,11.0,5.1 \mathrm{~Hz}, 1 \mathrm{H}$ ), $1.32(\mathrm{t}, J=7.1 \mathrm{~Hz}, 3 \mathrm{H}) \mathrm{ppm} .{ }^{13} \mathrm{C}$ NMR $\left(125 \mathrm{MHz}, \mathrm{d}_{6}\right.$-DMSO) $\delta 185.8,168.0,162.1,152.2,136.3,133.2$, 129.7, 127.9, 125.1, 122.0, 120.4, 117.5, 116.9, 116.2, 112.0, 111.2, 76.6, 76.3, 74.4, 62.8, 51.5, 47.7,

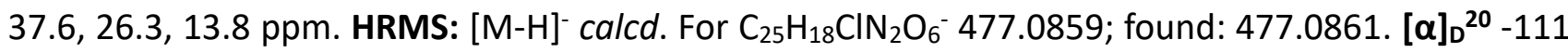
( $\mathrm{c}=0.25$ in $\mathrm{CHCl}_{3}$ ). The enantiomeric excess was determined by HPLC analysis on Daicel Chiralpak IB column $[n$-hexane $/ i-\mathrm{PrOH}=85 / 15,1 \mathrm{~mL} / \mathrm{min}], \lambda=210 \mathrm{~nm}, t_{\text {major }}=11.89 \mathrm{~min}, t_{\text {minor }}=13.95 \mathrm{~min}, \boldsymbol{e e}=$ $96 \%$. 


\section{Ethyl (5aR,7R,7aR,13aS,13bS)-6,6-dicyano-10,12-difluoro-7-hydroxy-8-oxo-5a,6,7,7a,8,13a,13b,14}

-octahydrochromeno[2,3-a]xanthene-7-carboxylate (4q)

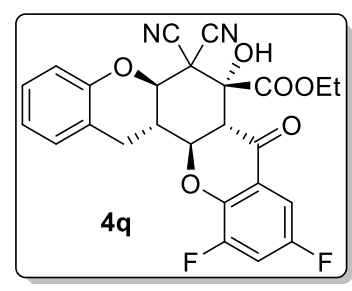

$\mathbf{4 q}$ was obtained as a white solid (43 $\mathrm{mg}, 90 \%)$ by General procedure $9 .{ }^{1} \mathbf{H}$ NMR $\left(500 \mathrm{MHz}, \mathrm{d}_{6}-\mathrm{DMSO}\right) \delta 8.03(\mathrm{~s}, 1 \mathrm{H}), 7.84-7.77(\mathrm{~m}, 1 \mathrm{H}), 7.42-7.36(\mathrm{~m}$, $1 \mathrm{H}), 7.29(\mathrm{~d}, J=7.5 \mathrm{~Hz}, 1 \mathrm{H}), 7.19(\mathrm{t}, J=7.7 \mathrm{~Hz}, 1 \mathrm{H}), 7.02-6.95(\mathrm{~m}, 2 \mathrm{H}), 4.77$ $(\mathrm{d}, J=10.9 \mathrm{~Hz}, 1 \mathrm{H}), 4.71(\mathrm{dd}, J=13.0,10.2 \mathrm{~Hz}, 1 \mathrm{H}), 4.49-4.31(\mathrm{~m}, 2 \mathrm{H}), 4.05$ (d, $J=13.0 \mathrm{~Hz}, 1 \mathrm{H}$ ), 3.30 (dd, $J=16.5,5.0 \mathrm{~Hz}, 1 \mathrm{H}$ ), 3.09 (dd, $J=16.3,11.4 \mathrm{~Hz}, 1 \mathrm{H}), 2.70-2.59(\mathrm{~m}$, 1H), $1.32\left(\mathrm{t}, J=7.1 \mathrm{~Hz}, 3 \mathrm{H}\right.$ ) ppm. ${ }^{13} \mathrm{C}$ NMR (125 MHz, $\mathrm{d}_{6}$-DMSO) $\delta 186.5,167.8,152.2,152.0,146.0$, $159.9,129.8,127.9,122.0,120.3,116.2,111.9,111.7,111.2,107.4,107.2,78.1,76.5,74.3,62.9$, 51.2, 47.7, 37.5, 26.3, 13.8 ppm. HRMS: [M-H] calcd. For $\mathrm{C}_{25} \mathrm{H}_{17} \mathrm{~F}_{2} \mathrm{~N}_{2} \mathrm{O}_{6}{ }^{-}$479.1060; found: 479.1055. $[\alpha]_{D}^{20}-98\left(c=0.25\right.$ in $\left.\mathrm{CHCl}_{3}\right)$. The enantiomeric excess was determined by HPLC analysis on Daicel Chiralpak IC column [ $n$-hexane $/ i-\mathrm{PrOH}=80 / 20,1 \mathrm{~mL} / \mathrm{min}$ ], $\lambda=210 \mathrm{~nm}, t_{\text {minor }}=6.35 \mathrm{~min}, t_{\text {major }}=8.66$ $\min , \boldsymbol{e} e=95 \%$.

ethyl

$(7 \mathrm{a} R, 9 R, 9 \mathrm{a} R, 15 \mathrm{aS}, 15 \mathrm{bS})-8,8-$ dicyano-9-hydroxy-10-oxo-7a,8,9,9a,10,15a,15b,16octahydrobenzo[ $[a]$ chromeno[3,2-j]xanthene-9-carboxylate $(4 r)$

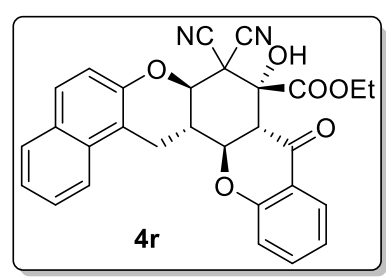

$4 \mathrm{r}$ was obtained as a white solid (42 $\mathrm{mg}, 85 \%$ ) by General procedure $9 .{ }^{1} \mathbf{H}$ NMR $\left(500 \mathrm{MHz}, \mathrm{d}_{6}-\mathrm{DMSO}\right) \delta 7.98(\mathrm{~s}, 1 \mathrm{H}), 7.96-7.90(\mathrm{~m}, 2 \mathrm{H}), 7.84-7.79$ (m, 2H), $7.73-7.67(m, 1 \mathrm{H}), 7.66-7.58(\mathrm{~m}, 1 \mathrm{H}), 7.46(\mathrm{t}, J=7.5 \mathrm{~Hz}, 1 \mathrm{H})$, $7.27(\mathrm{~d}, J=8.3 \mathrm{~Hz}, 1 \mathrm{H}), 7.23(\mathrm{~d}, J=8.9 \mathrm{~Hz}, 1 \mathrm{H}), 7.18(\mathrm{t}, J=7.5 \mathrm{~Hz}, 1 \mathrm{H}), 4.82$ $(\mathrm{d}, J=11.0 \mathrm{~Hz}, 1 \mathrm{H}), 4.72(\mathrm{dd}, J=12.9,10.2 \mathrm{~Hz}, 1 \mathrm{H}), 4.51-4.33(\mathrm{~m}, 2 \mathrm{H}), 3.90(\mathrm{~d}, J=13.0 \mathrm{~Hz}, 1 \mathrm{H})$, $3.76(\mathrm{dd}, J=16.7,5.4 \mathrm{~Hz}, 1 \mathrm{H}), 3.20(\mathrm{dd}, J=16.6,11.0 \mathrm{~Hz}, 1 \mathrm{H}), 2.82-2.72(\mathrm{~m}, 1 \mathrm{H}), 1.34(\mathrm{t}, J=7.1 \mathrm{~Hz}$, 3H) ppm. ${ }^{13} \mathrm{C}$ NMR (125 MHz, $\mathrm{d}_{6}$-DMSO) $\delta 187.8,168.0,160.6,149.8,137.1,132.0,129.2,128.5$, $128.3,127.0,126.8,124.2,122.3,122.2,120.0,118.2,117.8,112.9,112.1,111.3,77.3,76.6,74.3$, 62.8, 51.3, 47.6, 37.4, 23.3, 13.8 ppm. HRMS: [M-H] calcd. For $\mathrm{C}_{29} \mathrm{H}_{21} \mathrm{~N}_{2} \mathrm{O}_{6}{ }^{-}$493.1405; found: 493.1406. $[\alpha]_{D}^{20}-45\left(c=0.25\right.$ in $\mathrm{CHCl}_{3}$ ). The enantiomeric excess was determined by HPLC analysis on Daicel Chiralpak IC column [ $n$-hexane/i-PrOH $=80 / 20,1 \mathrm{~mL} / \mathrm{min}$ ], $\lambda=210 \mathrm{~nm}, t_{\text {minor }}=9.45 \mathrm{~min}$, $t_{\text {major }}=11.34 \mathrm{~min}, \boldsymbol{e e}=\mathbf{9 7 \%}$. 


\section{octahydrobenzo[ $h]$ chromeno[2,3-a]xanthene-7-carboxylate (4s)}

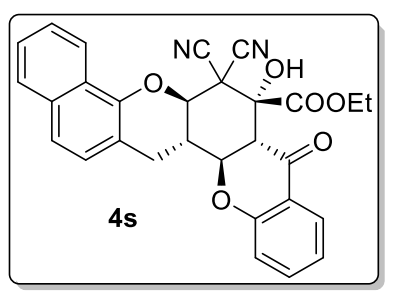

4s was obtained as a white solid (43 $\mathrm{mg}, 87 \%$ ) by General procedure $9 .{ }^{\mathbf{1}} \mathbf{H}$ NMR (500 MHz, d6-DMSO) $\delta 8.05(\mathrm{~d}, J=8.3 \mathrm{~Hz}, 1 \mathrm{H}), 7.99(\mathrm{~s}, 1 \mathrm{H}), 7.91$ (d, J $=8.0 \mathrm{~Hz}, 1 \mathrm{H}), 7.81(\mathrm{dd}, J=7.8,1.5 \mathrm{~Hz}, 1 \mathrm{H}), 7.71-7.65(\mathrm{~m}, 1 \mathrm{H}), 7.63-7.52$ (m, 3H), $7.38(\mathrm{~d}, J=8.4 \mathrm{~Hz}, 1 \mathrm{H}), 7.20-7.14(\mathrm{~m}, 2 \mathrm{H}), 4.94(\mathrm{~d}, J=10.9 \mathrm{~Hz}$, 1H), $4.68(\mathrm{dd}, J=12.9,10.2 \mathrm{~Hz}, 1 \mathrm{H}), 4.52-4.33(\mathrm{~m}, 2 \mathrm{H}), 3.91$ (d, J = $12.9 \mathrm{~Hz}, 1 \mathrm{H}), 3.45$ (dd, $J=16.6$, $5.2 \mathrm{~Hz}, 1 \mathrm{H}), 3.23(\mathrm{dd}, J=16.5,11.1 \mathrm{~Hz}, 1 \mathrm{H}), 2.82-2.70(\mathrm{~m}, 1 \mathrm{H}), 1.35(\mathrm{t}, J=7.1 \mathrm{~Hz}, 3 \mathrm{H}) \mathrm{ppm} .{ }^{13} \mathrm{C}$ NMR (125 MHz, $\mathrm{d}_{6}$-DMSO) $\delta 187.7,168.0,160.5,146.7,137.1,132.8,127.7,127.4,126.8,126.3$, 126.2 , 123.6, 122.2, 121.3, 120.0, 118.0, 115.0, 112.1, 111.4, 77.1, 76.6, 74.7, 62.8, 51.3, 47.8, 37.6,

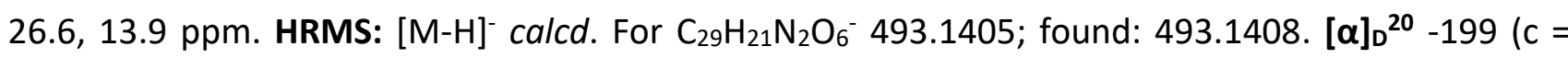
0.25 in $\mathrm{CHCl}_{3}$ ). The enantiomeric excess was determined by HPLC analysis on Daicel Chiralpak IC column $[n$-hexane $/ i-\mathrm{PrOH}=80 / 20,1 \mathrm{~mL} / \mathrm{min}], \lambda=210 \mathrm{~nm}, t_{\text {minor }}=8.78 \mathrm{~min}, t_{\text {major }}=10.9 \mathrm{~min}, \boldsymbol{e e}=$ $98 \%$.

Ethyl

(7aR,8R,9aR,15aS,15bS)-9,9-dicyano-8-hydroxy-7-oxo-7,7a,8,9,9a,15,15a,15boctahydrobenzo[c]chromeno[3,2-h]xanthene-8-carboxylate $(4 t)$

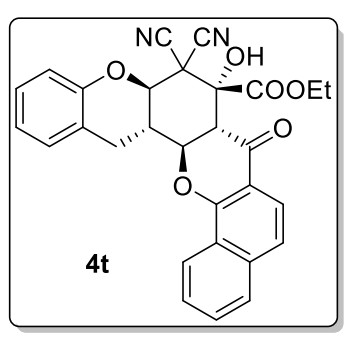

4t was obtained as a white solid (41 mg, 83\%) by General procedure $9 .{ }^{1} \mathbf{H}$ NMR (500 MHz, d 6 -DMSO) $\delta 8.43(\mathrm{~d}, J=8.2 \mathrm{~Hz}, 1 \mathrm{H}), 8.01-7.96(\mathrm{~m}, 2 \mathrm{H}), 7.80$ $-7.75(\mathrm{~m}, 1 \mathrm{H}), 7.75-7.67(\mathrm{~m}, 2 \mathrm{H}), 7.60(\mathrm{~d}, J=8.7 \mathrm{~Hz}, 1 \mathrm{H}), 7.35(\mathrm{~d}, J=7.5 \mathrm{~Hz}$, 1H), $7.21(t, J=7.3 \mathrm{~Hz}, 1 \mathrm{H}), 7.06-6.97(\mathrm{~m}, 2 \mathrm{H}), 4.88-4.73(\mathrm{~m}, 2 \mathrm{H}), 4.52-$ $4.31(\mathrm{~m}, 2 \mathrm{H}), 3.96(\mathrm{~d}, J=13.3 \mathrm{~Hz}, 1 \mathrm{H}), 3.64(\mathrm{dd}, J=16.4,4.9 \mathrm{~Hz}, 1 \mathrm{H}), 3.16$ (dd, $J=16.2,11.5 \mathrm{~Hz}, 1 \mathrm{H}), 2.82-2.69(\mathrm{~m}, 1 \mathrm{H}), 1.34(\mathrm{t}, J=7.1 \mathrm{~Hz}, 3 \mathrm{H}) \mathrm{ppm} .{ }^{13} \mathrm{C}$ NMR (125 MHz, d6-DMSO) $\delta 187.3,168.0,158.7,152.3,137.1,130.4,129.9,128.0,127.8,127.0,123.9,123.3,122.0,121.7$, $121.1,120.6,116.2,114.7,112.1,111.3,77.9,76.7,74.6,62.8,50.6,47.8,37.7,26.2,13.9$ ppm. HRMS: [M-H] ${ }^{-}$calcd. For $\mathrm{C}_{29} \mathrm{H}_{21} \mathrm{~N}_{2} \mathrm{O}_{6}{ }^{-} 493.1405$; found: 493.1409. [ $\left.\alpha\right]_{D}{ }^{20}-131$ ( $\mathrm{c}=0.25$ in $\mathrm{CHCl}_{3}$ ). The enantiomeric excess was determined by HPLC analysis on Daicel Chiralpak IC column $[n$-hexane $/ i-\mathrm{PrOH}=80 / 20,1 \mathrm{~mL} / \mathrm{min}], \lambda=210 \mathrm{~nm}, t_{\text {minor }}=10.52 \mathrm{~min}, t_{\text {major }}=11.91 \mathrm{~min}, \boldsymbol{e e}=\mathbf{9 8 \%}$. 


\section{octahydrobenzo[ $[a]$ chromeno[3,2-h]xanthene-6-carboxylate $(4 \mathrm{u})$}

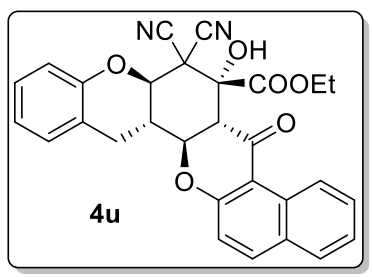

4u was obtained as a white solid ( $40 \mathrm{mg}, 81 \%)$ by General procedure $9 .{ }^{1} \mathbf{H}$ NMR (500 MHz, d 6 -DMSO) $\delta 9.29(\mathrm{~d}, J=8.5 \mathrm{~Hz}, 1 \mathrm{H}), 8.24(\mathrm{~d}, J=9.1 \mathrm{~Hz}$ 1H), $7.97(\mathrm{~d}, J=8.1 \mathrm{~Hz}, 1 \mathrm{H}), 7.93(\mathrm{~s}, 1 \mathrm{H}), 7.70(\mathrm{t}, J=7.8 \mathrm{~Hz}, 1 \mathrm{H}), 7.52(\mathrm{t}, J=$ $7.4 \mathrm{~Hz}, 1 \mathrm{H}), 7.34(\mathrm{~d}, J=9.0 \mathrm{~Hz}, 1 \mathrm{H}), 7.28(\mathrm{~d}, J=7.4 \mathrm{~Hz}, 1 \mathrm{H}), 7.21(\mathrm{t}, J=7.7$ $\mathrm{Hz}, 1 \mathrm{H}), 7.04-6.96(\mathrm{~m}, 2 \mathrm{H}), 4.80(\mathrm{~d}, J=10.9 \mathrm{~Hz}, 1 \mathrm{H}), 4.74(\mathrm{dd}, J=13.3,10.3 \mathrm{~Hz}, 1 \mathrm{H}), 4.53-4.33(\mathrm{~m}$, 2H), $3.95(\mathrm{~d}, J=13.4 \mathrm{~Hz}, 1 \mathrm{H}), 3.39(\mathrm{dd}, J=16.3,4.8 \mathrm{~Hz}, 1 \mathrm{H}), 3.13(\mathrm{dd}, J=16.2,11.5 \mathrm{~Hz}, 1 \mathrm{H}), 2.72-$ $2.60(\mathrm{~m}, 1 \mathrm{H}), 1.33(\mathrm{t}, J=7.1 \mathrm{~Hz}, 3 \mathrm{H}) \mathrm{ppm} .{ }^{13} \mathrm{C}$ NMR (125 MHz, $\mathrm{d}_{6}$-DMSO) $\delta$ 188.7, 168.2, 163.0, 152.3, $138.7,130.7,130.0,129.8,129.0,127.9,125.2,124.7,122.0,120.4,118.6,116.2,112.1,111.3$, 111.2, 76.8, 76.7, 74.6, 62.7, 51.5, 47.8, 37.5, 26.3, 13.9 ppm. HRMS: [M-H] calcd. For $\mathrm{C}_{29} \mathrm{H}_{21} \mathrm{~N}_{2} \mathrm{O}_{6}{ }^{-}$ 493.1405; found: 493.1405. [ $\alpha]_{\mathrm{D}}{ }^{20}-128$ ( $\mathrm{c}=0.25$ in $\left.\mathrm{CHCl}_{3}\right)$. The enantiomeric excess was determined by HPLC analysis on Daicel Chiralpak IC column [ $n$-hexane/i-PrOH $=80 / 20,1 \mathrm{~mL} / \mathrm{min}$ ], $\lambda=210 \mathrm{~nm}$, $t_{\text {minor }}=10.55 \mathrm{~min}, t_{\text {major }}=12.73 \mathrm{~min}, \boldsymbol{e e}=\mathbf{8 7 \%}$.

Ethyl (5aR,7R,7aR,13aS,13bS)-10-(4-acetoxyphenyl)-6,6-dicyano-7-hydroxy-8-oxo-5a,6,7,7a,8,13a, 13b,14-octahydrochromeno[2,3-a]xanthene-7-carboxylate (4v)

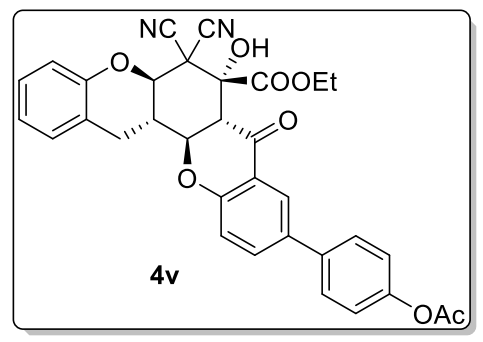

$4 \mathbf{v}$ was obtained as a white solid (50 $\mathrm{mg}, 86 \%$ ) by General procedure

9. ${ }^{1}$ H NMR (500 MHz, $\mathrm{d}_{6}$-DMSO) $\delta 8.04-7.91(\mathrm{~m}, 3 \mathrm{H}), 7.69(\mathrm{~d}, J=8.2$ $\mathrm{Hz}, 2 \mathrm{H}), 7.33-7.14(\mathrm{~m}, 5 \mathrm{H}), 7.04-6.94(\mathrm{~m}, 2 \mathrm{H}), 4.78(\mathrm{~d}, J=10.8 \mathrm{~Hz}$, $1 \mathrm{H}), 4.70-4.59(\mathrm{~m}, 1 \mathrm{H}), 4.49-4.31(\mathrm{~m}, 2 \mathrm{H}), 3.92(\mathrm{~d}, J=13.0 \mathrm{~Hz}, 1 \mathrm{H})$, $3.41-3.35(\mathrm{~m}, 1 \mathrm{H}), 3.15-3.04(\mathrm{~m}, 1 \mathrm{H}), 2.70-2.56(\mathrm{~m}, 1 \mathrm{H}), 2.29(\mathrm{~s}$, 3H), $1.33(\mathrm{t}, J=7.0 \mathrm{~Hz}, 3 \mathrm{H}) \mathrm{ppm} .{ }^{13} \mathrm{C}$ NMR $\left(125 \mathrm{MHz}, \mathrm{d}_{6}\right.$-DMSO) $\delta 187.7,169.2,168.0,159.9,152.3$, $150.1,136.0,135.3,133.3,129.7,127.9,127.5,124.1,122.5,122.0,120.4,120.2,118.8,116.2$, 112.1, 111.3, 77.1, 76.6, 74.5, 62.9, 51.2, 47.7, 37.6, 26.4, 20.9, 13.8 ppm. HRMS: [M-H]' calcd. For $\mathrm{C}_{33} \mathrm{H}_{25} \mathrm{~N}_{2} \mathrm{O}_{8}^{-}$577.1616; found: 577.1615. [ $\left.\alpha\right]_{\mathrm{D}}{ }^{20}-95$ ( $\mathrm{c}=0.25$ in $\mathrm{CHCl}_{3}$ ). The enantiomeric excess was determined by HPLC analysis on Daicel Chiralpak IC column [ $n$-hexane $/ \mathrm{i}-\mathrm{PrOH}=80 / 20,1 \mathrm{~mL} / \mathrm{min}$ ], $\lambda$ $=210 \mathrm{~nm}, t_{\text {minor }}=29.51 \mathrm{~min}, t_{\text {major }}=38.12 \mathrm{~min}, \boldsymbol{e e}=\mathbf{9 3} \%$. 
Ethyl (5aR,7R,7aR,13aS,13bS)-6,6-dicyano-10-((E)-3-(dimethylamino)acryloyl)-7,11-dihydroxy-8oxo-5a,6,7,7a,8,13a,13b,14-octahydrochromeno[2,3-a]xanthene-7-carboxylate (4w)

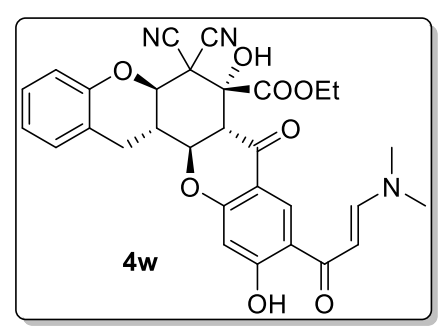

4w was obtained as a white solid (44 mg, 79\%) by General procedure 9. ${ }^{1}$ H NMR (500 MHz, d6-DMSO) $\delta 15.33(\mathrm{~s}, 1 \mathrm{H}), 8.25(\mathrm{~s}, 1 \mathrm{H}), 7.85$ (d, $J=$ $12.0 \mathrm{~Hz}, 1 \mathrm{H}), 7.21-7.11(\mathrm{~m}, 2 \mathrm{H}), 7.03-6.93(\mathrm{~m}, 2 \mathrm{H}), 6.43(\mathrm{~s}, 1 \mathrm{H}), 5.69$ $(\mathrm{d}, J=12.0 \mathrm{~Hz}, 1 \mathrm{H}), 4.67-4.58(\mathrm{~m}, 1 \mathrm{H}), 4.53-4.43(\mathrm{~m}, 2 \mathrm{H}), 4.39(\mathrm{dd}, J$ $=12.4,10.3 \mathrm{~Hz}, 1 \mathrm{H}), 3.67(\mathrm{~d}, J=12.7 \mathrm{~Hz}, 1 \mathrm{H}), 3.46$ (dd, $J=15.8,4.2 \mathrm{~Hz}$, $1 \mathrm{H}), 3.18(\mathrm{~s}, 3 \mathrm{H}), 2.97(\mathrm{~s}, 3 \mathrm{H}), 2.85-2.70(\mathrm{~m}, 2 \mathrm{H}), 1.43(\mathrm{t}, J=7.1 \mathrm{~Hz}, 3 \mathrm{H}) \mathrm{ppm} .{ }^{13} \mathrm{C}$ NMR (125 MHz, $\mathrm{d}_{6}$-DMSO) $\delta$ 190.0, 186.3, 171.3, 169.0, 164.5, 155.6, 152.8, 129.8, 129.7, 128.4, 122.5, 119.5, 117.2, $116.8,112.4,111.2,110.9,104.9,89.4,78.0,75.9,75.3,65.1,50.7,47.7,45.8,37.9,37.9,27.9,14.2$

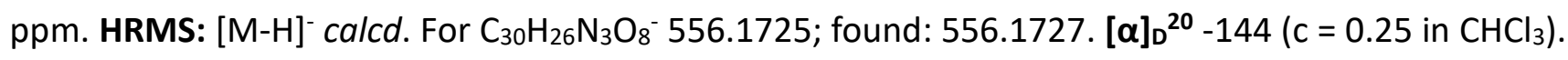
The enantiomeric excess was determined by HPLC analysis on Daicel Chiralpak IA column [n-hexane $/ \mathrm{i}$ - $\mathrm{PrOH} / \mathrm{CH}_{2} \mathrm{Cl}_{2}=70 / 25 / 5,1 \mathrm{~mL} / \mathrm{min}$ ], $\lambda=260 \mathrm{~nm}, t_{\text {minor }}=16.92 \mathrm{~min}, t_{\text {major }}=30.95 \mathrm{~min}$, ee $=98 \%$.

Ethyl

$(6 \mathrm{a} R, 7 R, 8 \mathrm{a} R, 14 \mathrm{a} S, 14 \mathrm{bS})-8,8-$ dicyano-7-hydroxy-6-oxo-3-tosyl-6,6a,7,8,8a,14,14a,14boctahydro-3H-chromeno[2',3':7,8]chromeno[2,3-e]indole-7-carboxylate (4x)

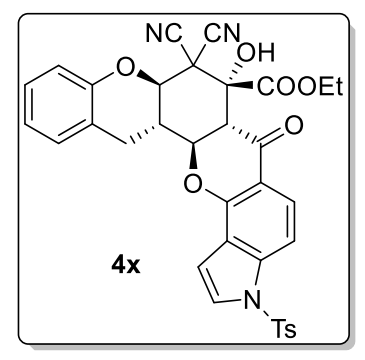

4x was obtained as a white solid (58 $\mathrm{mg}, 91 \%$ ) by General procedure $9 .{ }^{1} \mathbf{H}$ NMR (500 MHz, $\mathrm{d}_{6}$-DMSO) $\delta 7.94-7.88(\mathrm{~m}, 4 \mathrm{H}), 7.73-7.65(\mathrm{~m}, 2 \mathrm{H}), 7.41$ (d, $J=8.1 \mathrm{~Hz}, 2 \mathrm{H}), 7.26(\mathrm{~d}, J=7.6 \mathrm{~Hz}, 1 \mathrm{H}), 7.19(\mathrm{t}, J=7.7 \mathrm{~Hz}, 1 \mathrm{H}), 7.13(\mathrm{~d}, J=3.4$ $\mathrm{Hz}, 1 \mathrm{H}), 7.04-6.92(\mathrm{~m}, 2 \mathrm{H}), 4.76(\mathrm{~d}, J=10.9 \mathrm{~Hz}, 1 \mathrm{H}), 4.68(\mathrm{dd}, J=12.4,10.8$ $H z, 1 H), 4.46-4.30(m, 2 H), 3.89(d, J=13.0 \mathrm{~Hz}, 1 \mathrm{H}), 3.52(\mathrm{dd}, J=16.4,4.6$ $\mathrm{Hz}, 1 \mathrm{H}), 3.05(\mathrm{dd}, J=16.1,11.8 \mathrm{~Hz}, 1 \mathrm{H}), 2.69-2.56(\mathrm{~m}, 1 \mathrm{H}), 2.33(\mathrm{~s}, 3 \mathrm{H}), 1.31(\mathrm{t}, J=7.1 \mathrm{~Hz}, 3 \mathrm{H}) \mathrm{ppm}$. ${ }^{13}$ C NMR (125 MHz, d6-DMSO) $\delta$ 186.8, 168.0, 155.0, 152.3, 146.1, 138.9, 133.8, 130.4, 129.7, 127.8, 127.4, 126.9, 122.9, 121.9, 120.5, 119.8, 116.2, 114.7, 112.1, 111.3, 107.7, 106.5, 77.7, 76.6, 74.5, 62.8, 50.8, 47.7, 37.6, 26.1, 21.0, 13.8 ppm. HRMS: [M-H] calcd. For $\mathrm{C}_{34} \mathrm{H}_{26} \mathrm{~N}_{3} \mathrm{O}_{8} \mathrm{~S}^{-}$636.1446; found: 636.1441. $[\alpha]_{D}{ }^{20}-87\left(c=0.25\right.$ in $\mathrm{CHCl}_{3}$ ). The enantiomeric excess was determined by HPLC analysis on Daicel Chiralpak IC column $[n$-hexane $/ i-\mathrm{PrOH}=80 / 20,1 \mathrm{~mL} / \mathrm{min}], \lambda=230 \mathrm{~nm}, t_{\text {minor }}=38.64 \mathrm{~min}$, $t_{\text {major }}=50.43 \mathrm{~min}, \boldsymbol{e e}=\mathbf{9 1 \%}$. 
Diethyl 10,10'-(propane-2,2-diyl)(5aR,5a'R,7R,7aR,7'R,7a'R,13aS,13bS,13a'S,13b'S)-bis(6,6-dicyano -7-hydroxy-8-oxo-5a,6,7,7a,8,13a,13b,14-octahydrochromeno[2,3-a]xanthene-7-carboxylate) (6)

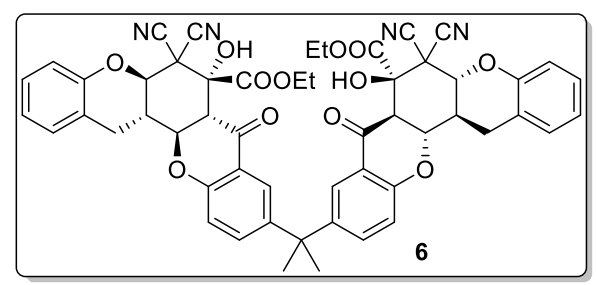

6 was obtained as a white solid (33 mg, 71\%) by General procedure 9. ${ }^{1} \mathrm{H}$ NMR (500 MHz, $\mathrm{d}_{6}$-DMSO) $\delta 7.87(\mathrm{~s}, 2 \mathrm{H}), 7.62$ (d, $J=2.3 \mathrm{~Hz}, 2 \mathrm{H}), 7.49$ (dd, $J=8.8,2.3 \mathrm{~Hz}, 2 \mathrm{H}), 7.25$ (d, $J=7.5$ $\mathrm{Hz}, 2 \mathrm{H}), 7.19(\mathrm{t}, J=7.7 \mathrm{~Hz}, 2 \mathrm{H}), 7.11(\mathrm{~d}, J=8.7 \mathrm{~Hz}, 2 \mathrm{H}), 6.98(\mathrm{dd}$, $J=14.7,7.7 \mathrm{~Hz}, 4 \mathrm{H}), 4.74(\mathrm{~d}, J=10.9 \mathrm{~Hz}, 2 \mathrm{H}), 4.58(\mathrm{dd}, J=12.8$, $10.3 \mathrm{~Hz}, 2 \mathrm{H}), 4.48-4.39(\mathrm{~m}, 2 \mathrm{H}), 4.37-4.27(\mathrm{~m}, 2 \mathrm{H}), 3.84(\mathrm{~d}, J=12.9 \mathrm{~Hz}, 2 \mathrm{H}), 3.33-3.28(\mathrm{~m}, 3 \mathrm{H})$, $3.06(\mathrm{dd}, J=16.2,11.5 \mathrm{~Hz}, 2 \mathrm{H}), 2.58(\mathrm{qd}, J=10.8,5.1 \mathrm{~Hz}, 2 \mathrm{H}), 1.64(\mathrm{~s}, 6 \mathrm{H}), 1.32(\mathrm{t}, J=7.1 \mathrm{~Hz}, 6 \mathrm{H})$ ppm. ${ }^{13} \mathrm{C}$ NMR $\left(125 \mathrm{MHz}, \mathrm{d}_{6}\right.$-DMSO) $\delta 187.8,168.0,158.8,152.2,143.4,135.9,129.7,127.8,123.5$, $121.9,120.4,119.3,118.1,116.1,112.0,111.2$, 77.1, 76.6, 74.5, 62.8, 51.2, 47.7, 41.7, 37.5, 30.0,

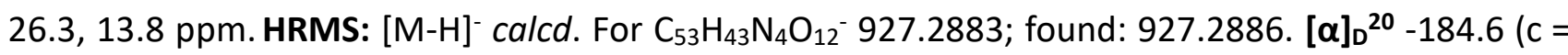
0.8 in $\mathrm{CHCl}_{3}$ ). The enantiomeric excess was determined by HPLC analysis on Daicel Chiralpak IC column $\left[n\right.$-hexane $/ i-\mathrm{PrOH} / \mathrm{CH}_{2} \mathrm{Cl}_{2}=75 / 20 / 5,1 \mathrm{~mL} / \mathrm{min}$ ], $\lambda=230 \mathrm{~nm}, t_{\text {minor }}=8.57 \mathrm{~min}, t_{\text {major }}=10.35$ $\min , \boldsymbol{e e}=\mathbf{9 0 \%}$. 
Triethyl 10,10',10"-(ethane-1,1,1-triyl)(5aR,5a'R,5a"R,7R,7aR,7'R,7a'R,7"R,7a"R,13aS,13bS,13a'S, 13b'S,13a"S,13b"S)-tris(6,6-dicyano-7-hydroxy-8-oxo-5a,6,7,7a,8,13a,13b,14-octahydrochromeno[ 2,3-a]xanthene-7-carboxylate) (8)

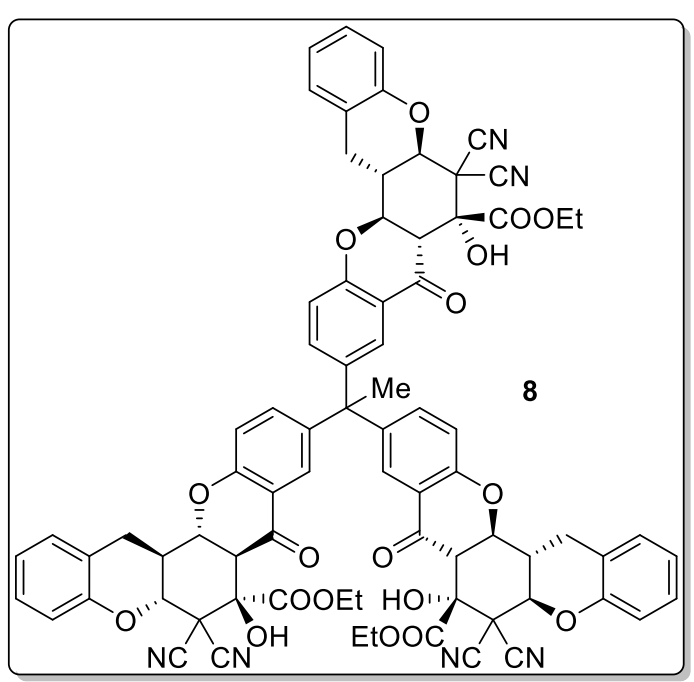

8 was obtained as a white solid ( $22 \mathrm{mg}, \mathrm{dr}=4: 1,48 \%)$ by General procedure 10. After recrystallization in diethyl ether to give diastereomerically pure product $\mathbf{8}$ (12mg, dr $>20: 1,28 \%)$ as a white solid. ${ }^{1}$ H NMR (500 $\left.\mathrm{MHz}, \mathrm{d}_{6}-\mathrm{DMSO}\right) \delta 7.87(\mathrm{~s}, 3 \mathrm{H}), 7.42(\mathrm{~d}, J=8.8 \mathrm{~Hz}, 3 \mathrm{H})$, $7.35(\mathrm{~d}, J=2.0 \mathrm{~Hz}, 3 \mathrm{H}), 7.26(\mathrm{~d}, J=7.5 \mathrm{~Hz}, 3 \mathrm{H}), 7.19$ (t, $J$ $=8.2 \mathrm{~Hz}, 6 \mathrm{H}), 6.99(\mathrm{dd}, J=16.4,8.0 \mathrm{~Hz}, 6 \mathrm{H}), 4.75(\mathrm{~d}, J=$ $10.9 \mathrm{~Hz}, 3 \mathrm{H}), 4.61$ (dd, $J=12.6,10.5 \mathrm{~Hz}, 3 \mathrm{H}), 4.45-$ $4.36(\mathrm{~m}, 3 \mathrm{H}), 4.33-4.24(\mathrm{~m}, 3 \mathrm{H}), 3.88(\mathrm{~d}, \mathrm{~J}=12.9 \mathrm{~Hz}$,

3H), $3.37-3.30(\mathrm{~m}, 3 \mathrm{H}), 3.16-3.04(\mathrm{~m}, 3 \mathrm{H}), 2.60(\mathrm{dt}, J=15.9,10.7 \mathrm{~Hz}, 3 \mathrm{H}), 2.13(\mathrm{~s}, 3 \mathrm{H}), 1.30(\mathrm{t}, J=$ $7.1 \mathrm{~Hz}, 9 \mathrm{H}$ ) ppm. ${ }^{13} \mathrm{C}$ NMR (125 MHz, dmso) $\delta$ 187.7, 167.9, 159.1, 152.2, 141.4, 137.1, 129.7, 127.8, $125.3,122.0,120.4,119.2,118.3,116.1,112.0,111.2,77.1,76.5,74.5,62.8,51.1,47.7,37.5,26.8$, 26.3, 14.0, 13.8 ppm. HRMS: [M-H] calcd. For $\mathrm{C}_{77} \mathrm{H}_{59} \mathrm{~N}_{6} \mathrm{O}_{18}{ }^{-}$1355.3891; found: 1355.3887. [ $\left.\alpha\right]_{D}{ }^{20}$ -56.6 ( $\mathrm{c}=0.8$ in $\mathrm{CHCl}_{3}$ ). The enantiomeric excess was determined by HPLC analysis on Daicel Chiralpak IA column [n-hexane/i-PrOH $/ \mathrm{CH}_{2} \mathrm{Cl}_{2}=65 / 30 / 5,1 \mathrm{~mL} / \mathrm{min}$ ], $\lambda=230 \mathrm{~nm}, t_{\text {minor }}=13.59 \mathrm{~min}$, $t_{\text {major }}=20.91 \mathrm{~min}, \boldsymbol{e e}=\mathbf{9 8 \%}$. 


\section{Further Research of Catalysts ${ }^{[a]}$}

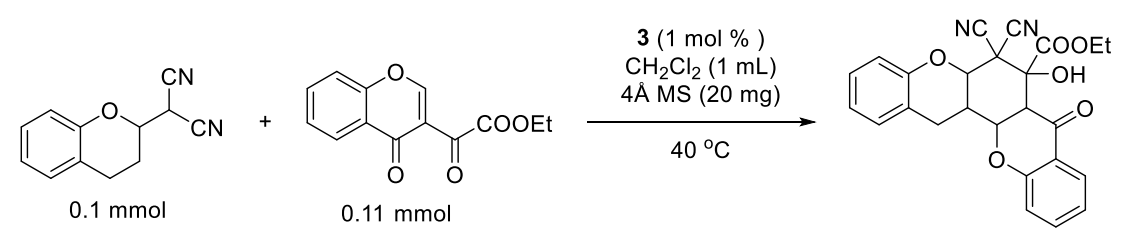

(RRS)-3

(RRRR)-3<smiles>CN(C)N[C@H]1CCCC[C@H]1NC(=O)N[C@H](C(=O)NCC(=O)NCc1cc(C(F)(F)F)cc(C(F)(F)F)c1)C(C)(C)C(C)(C)C</smiles>

Table 2:

\begin{tabular}{ccccc} 
entry & Catalyst & conditions & Yield (\%) & ee (\%) \\
\hline 1 & $(R R S S)-3$ & $8 \mathrm{~h}$ & 86 & 93 \\
\hline 2 & $(R R S R)-3$ & $8 \mathrm{~h}$ & 84 & 87 \\
\hline 3 & $(R R R S)-3$ & $8 \mathrm{~h}$ & 62 & 58 \\
\hline 4 & $(R R R R)-3$ & $8 \mathrm{~h}$ & 48 & 26 \\
\hline 5 & $(S S R R)-3$ & $8 \mathrm{~h}$ & 82 & -91 \\
\hline 6 & $(R R S S)-3-\mathrm{Me}$ & $8 \mathrm{~h}$ & 83 & 91 \\
\hline 7 & $(R R S)-3 \mathbf{a}$ & $8 \mathrm{~h}$ & 43 & 68 \\
\hline 8 & $(R R S)-3 \mathbf{b}$ & $8 \mathrm{~h}$ & 38 & 70
\end{tabular}

[a] If there is no special note, all reactions were performed by the method of general procedure 8 . 
In order to further understand the influence of catalyst configuration, hydrogen bond and steric hindrance effect on the reaction. A series of catalysts were synthesized and applied in the reaction. From the results of table 1 , we could see that:

First, the catalyst configuration, especially configuration of the first tertiary butyl group had great influence on the catalytic efficiency and ee value of products (entries 1-4). But if the configurations of cyclohexanediamine and dipeptide were all reversed, we could also get product with excellent ee value and good yield (entry 5).

Second, when the second amide of catalyst was protected by methyl group, it could still catalyze this reaction very efficiently (entry 6 ). This indicated that the hydrogen bond on the second amide might not participate in the catalytic system or have little influence on the ee value of products.

Finally, when we replaced any of the tertiary leucine of the catalyst with glycine, the ee values of the products were greatly reduced, which indicated that the steric hindrance effects of these two tert-butyl groups played equally important role in the catalytic process. 


\section{One Pot and Large Scale}

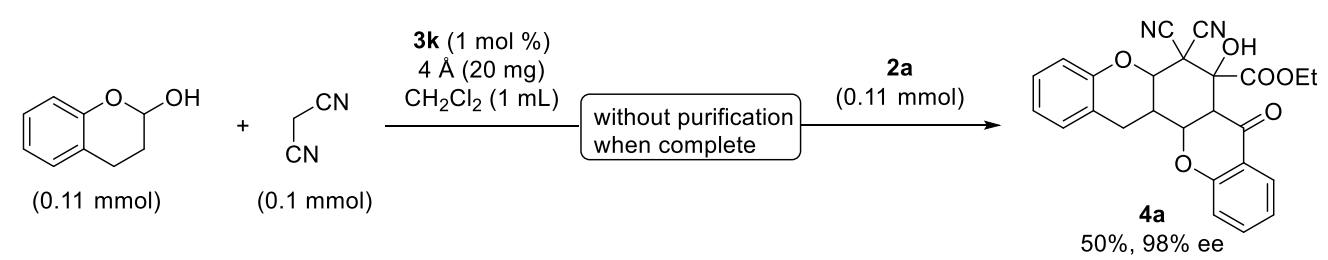

One pot experiment: To a solution of lactol (16.5 mg, $0.11 \mathrm{mmol})$, catalyst 3k (0.65 mg, $1 \mathrm{~mol} \%)$ and $4 \AA \mathrm{MS}(20 \mathrm{mg})$ in $\mathrm{CH}_{2} \mathrm{Cl}_{2}(1 \mathrm{~mL})$ was added malononitrile $(6.6 \mathrm{mg}, 0.1 \mathrm{mmol})$. The reaction was performed at $25^{\circ} \mathrm{C}$ until malononitrile was consumed completely. Then $2 \mathrm{a}(27 \mathrm{mg}, 0.11 \mathrm{mmol}$ ) was added and the reaction was allowed to stirred at $40{ }^{\circ} \mathrm{C}$ overnight. After purified by a flash column chromatography on silica gel, product 4 a ( $22 \mathrm{mg}, 50 \%$ yield) was obtained with $98 \%$ ee.

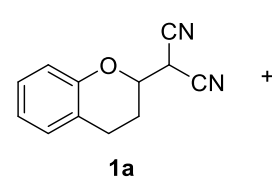

(198 mg, $1 \mathrm{mmol})$

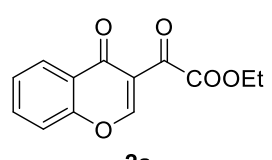

$(270 \mathrm{mg}, 1.1 \mathrm{mmol})$

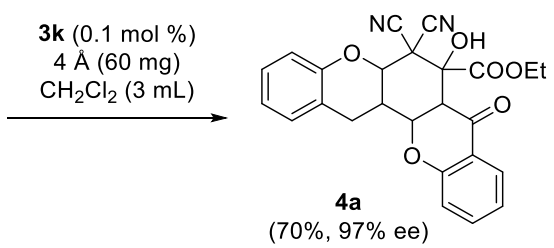

$(70 \%, 97 \%$ ee $)$

Large scale: To a solution of $1 \mathbf{a}(198 \mathrm{mg}, 1 \mathrm{mmol})$, catalyst $3 \mathbf{k}(0.1 \mathrm{~mol} \%)$ and $4 \AA \mathrm{MS}(60 \mathrm{mg})$ in $\mathrm{CH}_{2} \mathrm{Cl}_{2}(3 \mathrm{~mL})$ was added $2 \mathrm{a}(270 \mathrm{mg}, 1.1 \mathrm{mmol})$. The reaction was performed at $40{ }^{\circ} \mathrm{C}$ for $20 \mathrm{~h}$ when 1a was consumed completely. After purified by a flash column chromatography on silica gel, product 4 a (310 $\mathrm{mg}, 70 \%$ yield) was obtained with $97 \%$ ee. 


\section{Proposed Mechanism}

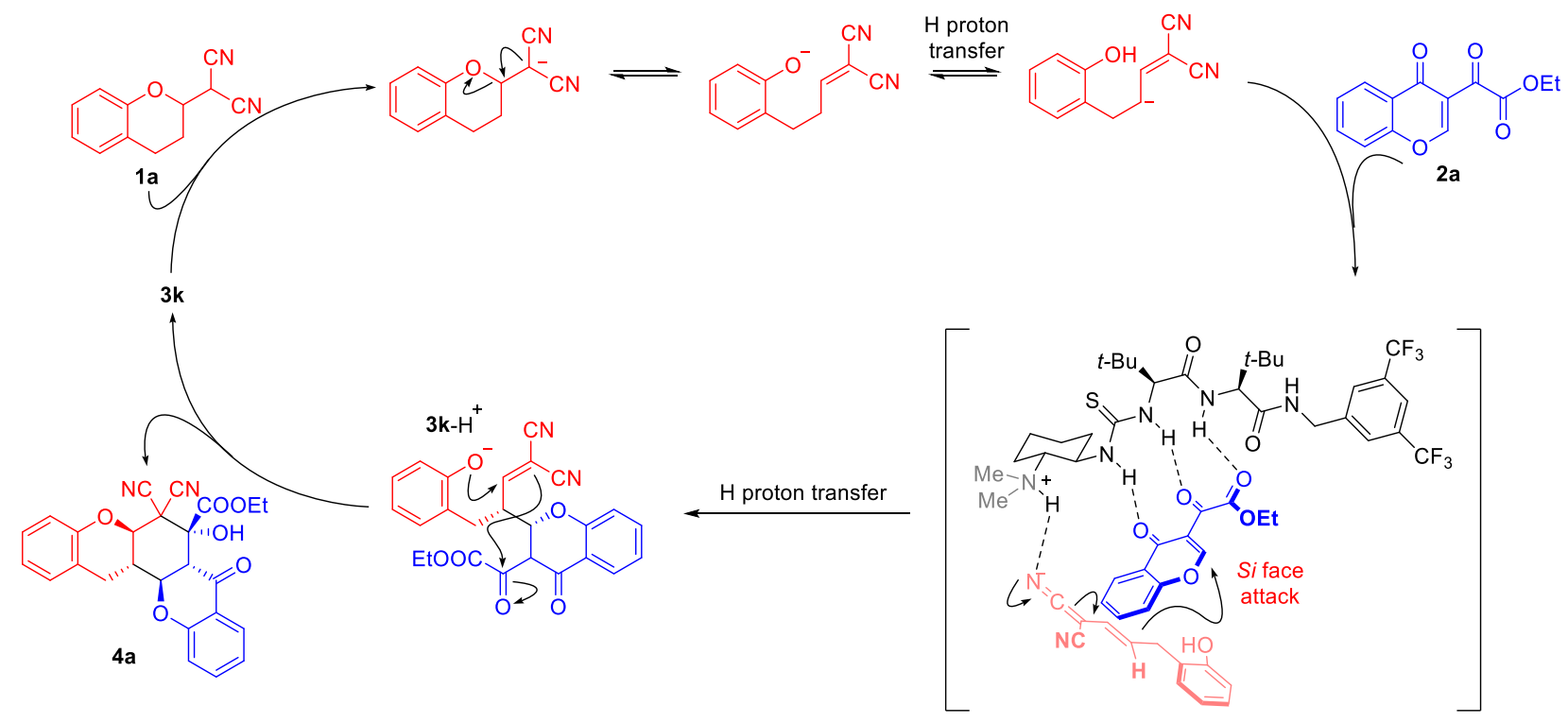

Proposed mechanism: Under optimal conditions, the proton at $\alpha$-position of cyano group was removed by $\mathbf{3 k}$ to give the phenolic oxyanion intermediate via E1cB elimination process. After proton transfer, it was activated and nucleophilic attacked substrate $\mathbf{2 a}$ from Si-face, which was activated by $\mathbf{3 k}$ through $\mathrm{H}$-bonding interaction. After proton transfer and followed by intramolecular oxa-Michael addition and aldol-type reaction, product $\mathbf{4 a}$ was obtained and $\mathbf{3} \mathbf{k}$ was recycled in the reaction. 


\section{X-ray Structure Determination}

\section{Single Crystal X-Ray Diffraction Data Single crystal X-ray structure (ORTEP) of rac-4a.}

X-ray crystallographic data have been deposited in the Cambridge Crystallographic Data Centre database (http://www.ccdc.cam.ac.uk/ data_request/cif.) under accession code CCDC 1943632.
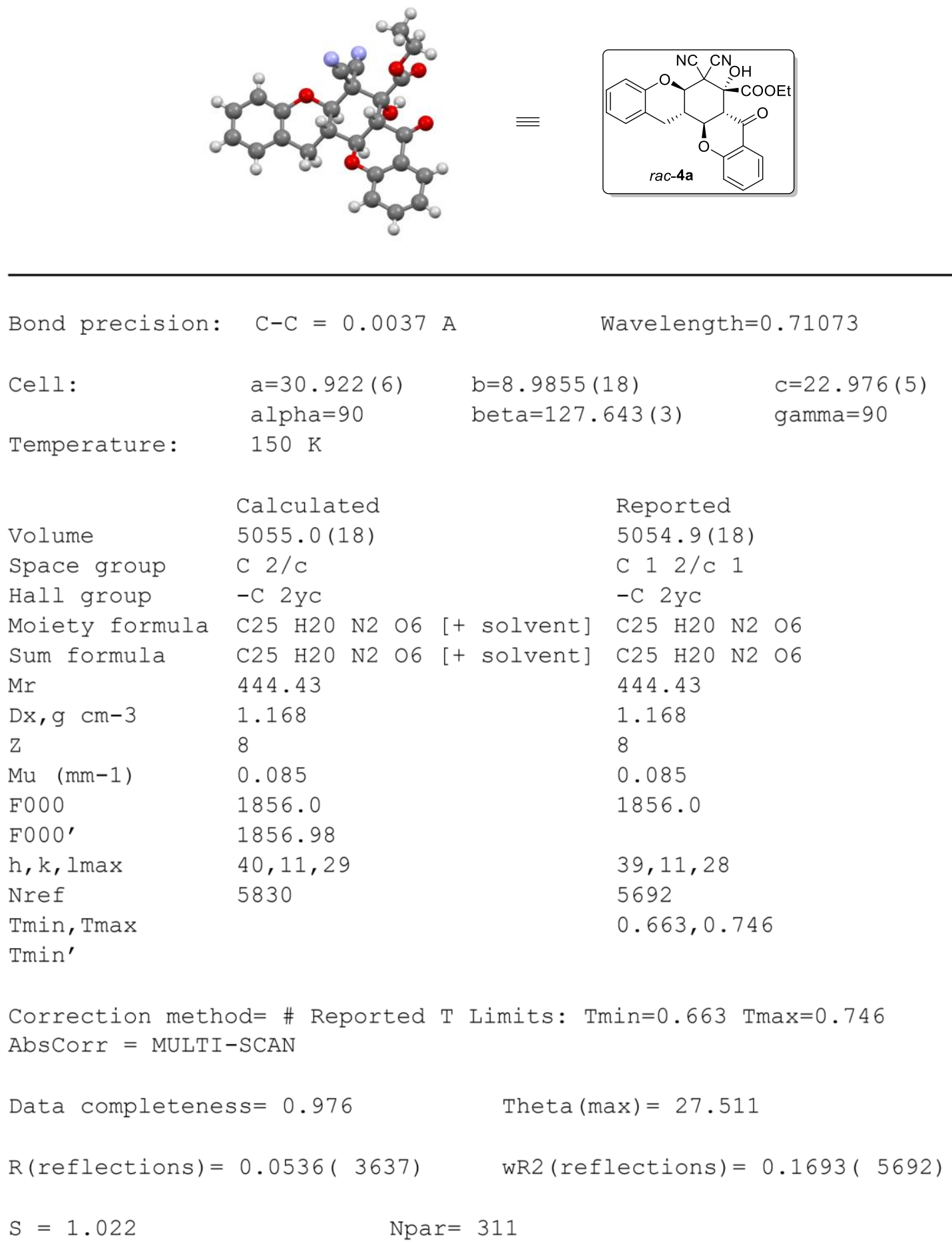
To assign the absolute configuration of $\mathbf{4 a}$, electronic circular dichroism (ECD) spectra were generated on the basis of quantum-chemical calculations. The ECD spectrum for $(2 R, 3 R, 7 S, 8 S$, 9R)-1 agrees well with that measured for $\mathbf{4 a}$. Thus, the absolute configuration of $\mathbf{4 a}$ was determined as $2 R, 3 R, 7 S, 8 S, 9 R$.

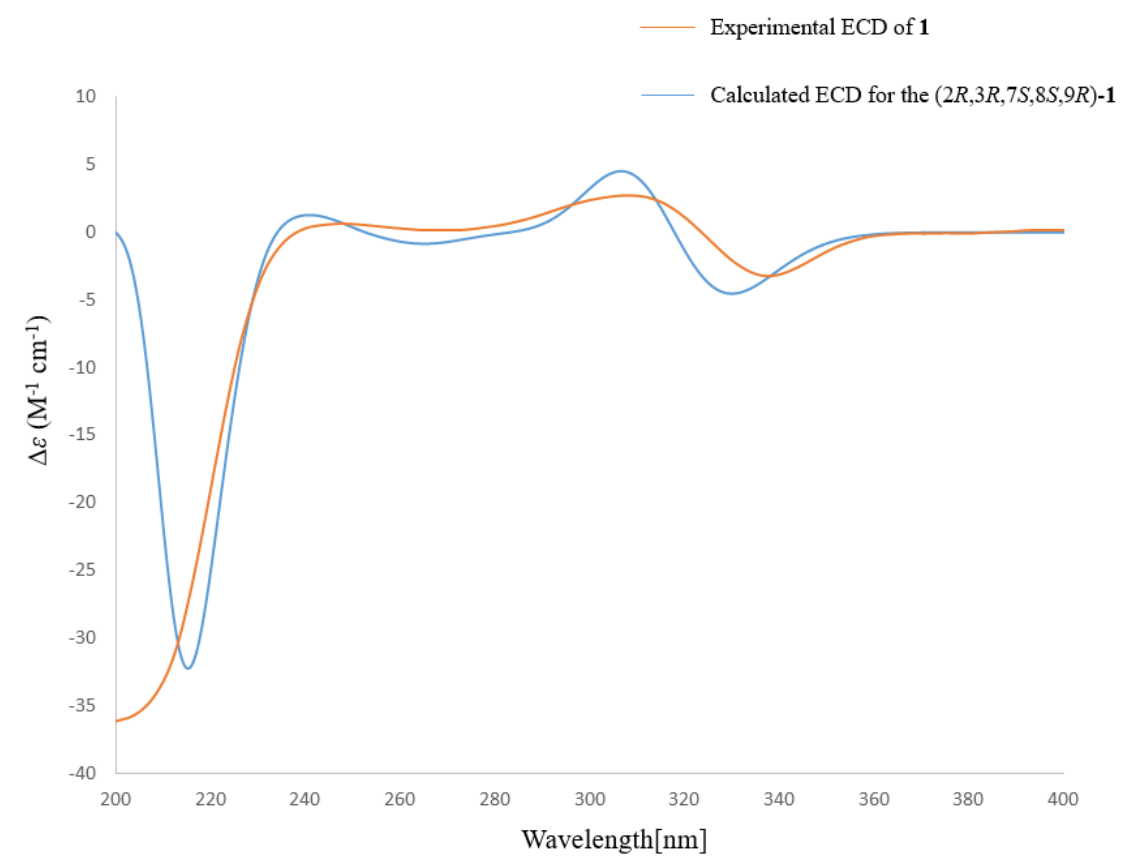

Computation Section. Conformational searches were run by employing the "systematic" procedure implemented in Spartan'14 using MMFF (Merck molecular force field). All MMFF minima were reoptimized with DFT calculations at the B3LYP/6-31+G(d) level using the Gaussian09 program. The geometry was optimized starting from various initial conformations, with vibrational frequency calculations confirming the presence of minima. Time-dependent DFT (TDDFT) calculations were performed on the six lowest-energy conformations for $4 a$ ( $>5 \%$ population) for each configuration using 20 excited states and using a polarizable continuum model (PCM) for MeOH. ECD spectra were generated using the program SpecDis by applying a Gaussian band shape with $0.15 \mathrm{eV}$ width for $4 \mathrm{a}$ from dipole length rotational strengths. The dipole velocity forms yielded negligible differences. The spectra of the conformers were combined using Boltzmann weighting, with the lowest-energy conformations accounting for about $99 \%$ of the weights for $\mathbf{4 a}$. The calculated spectra were shifted by $5 \mathrm{~nm}$ for $4 \mathrm{a}$ to facilitate comparison to the experimental data. 


\section{NMR Spectra and HPLC Traces}
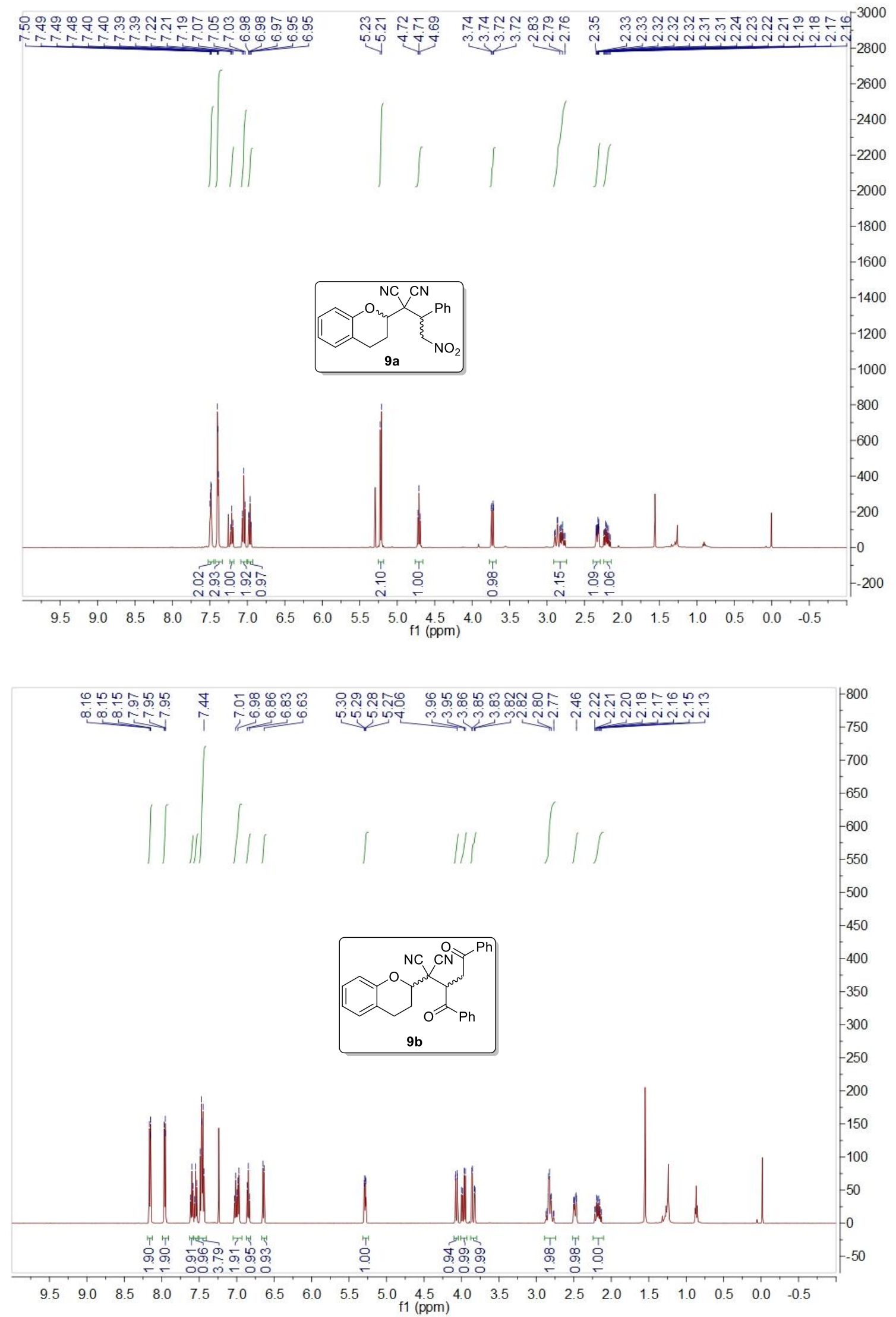

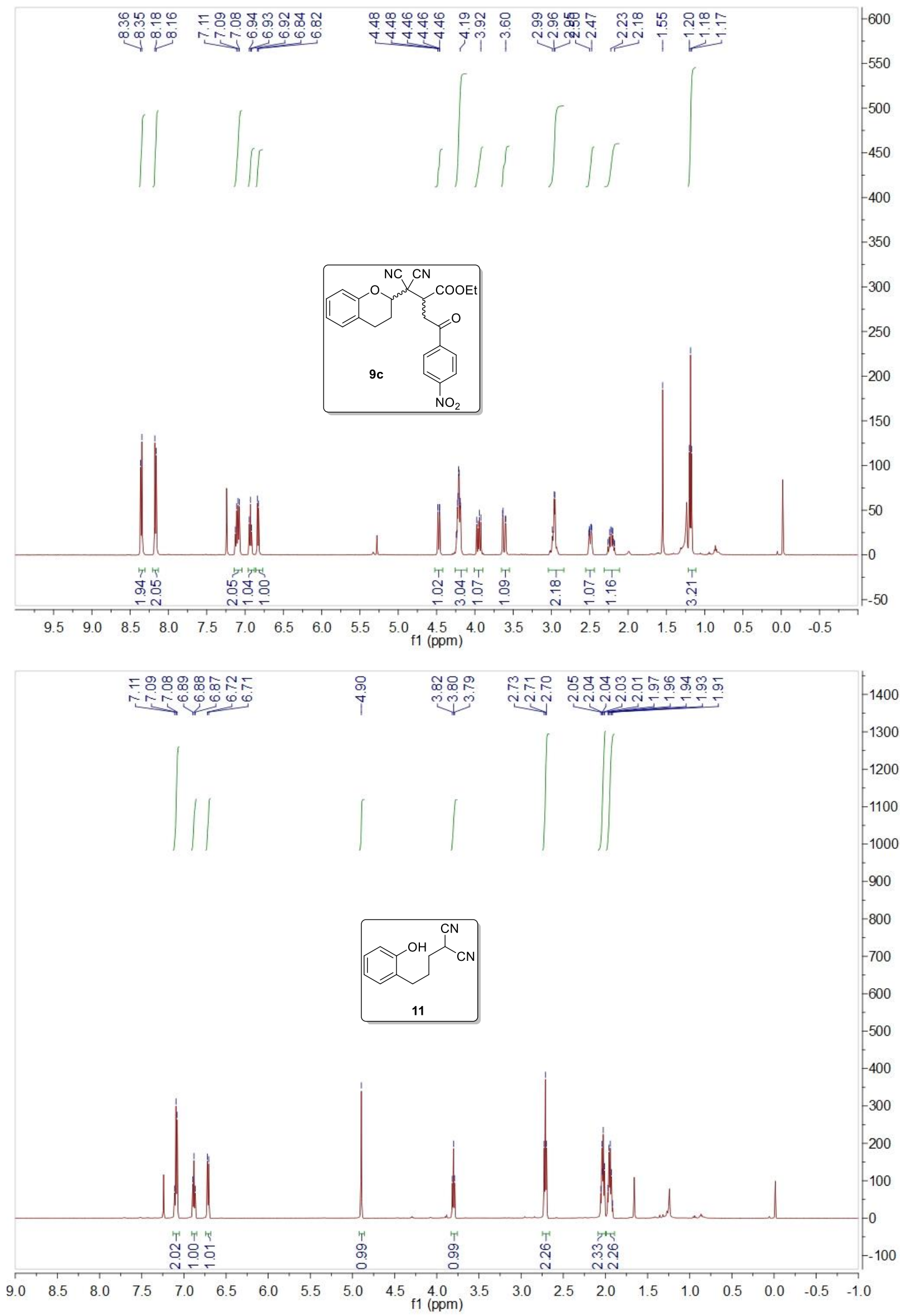

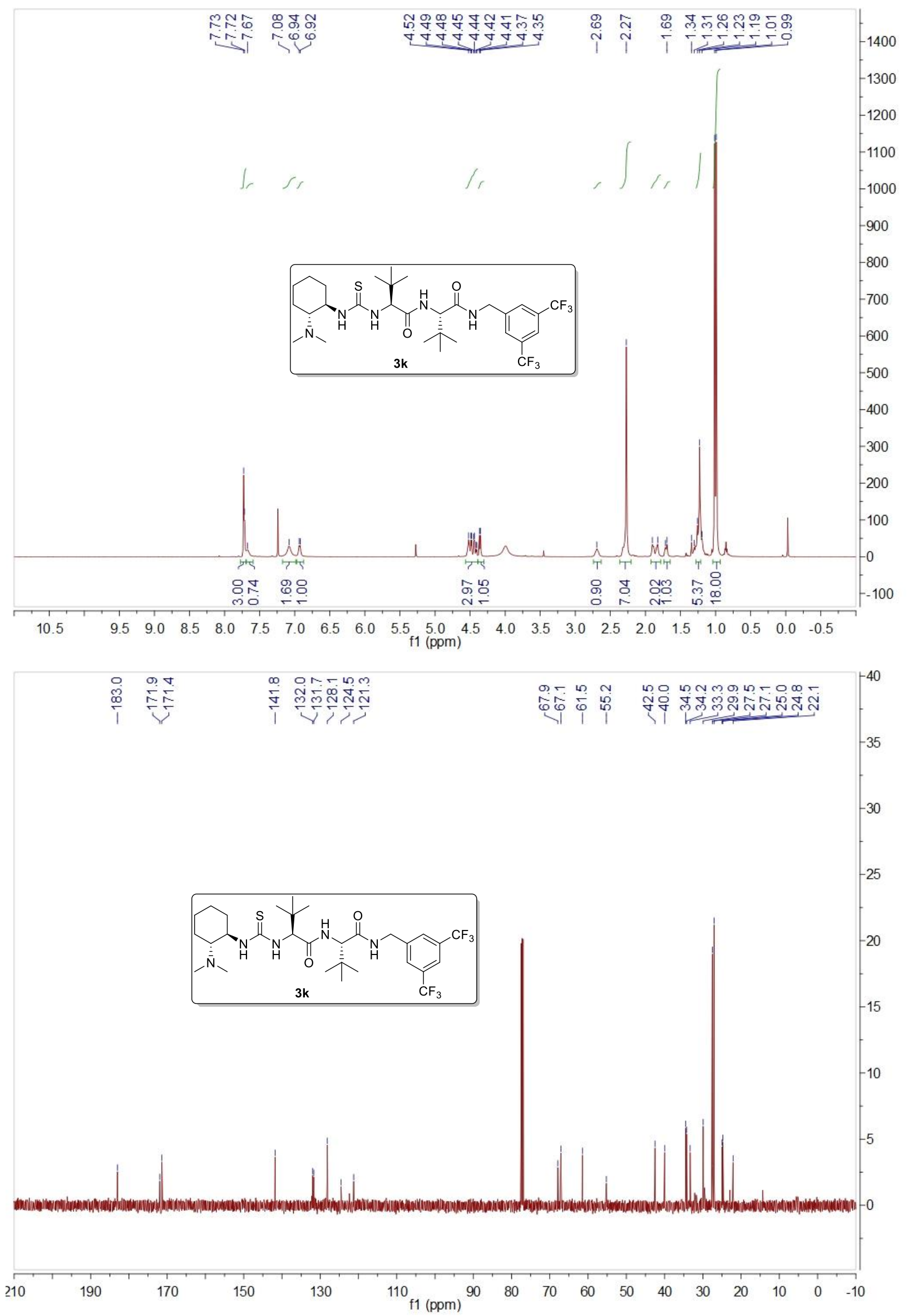

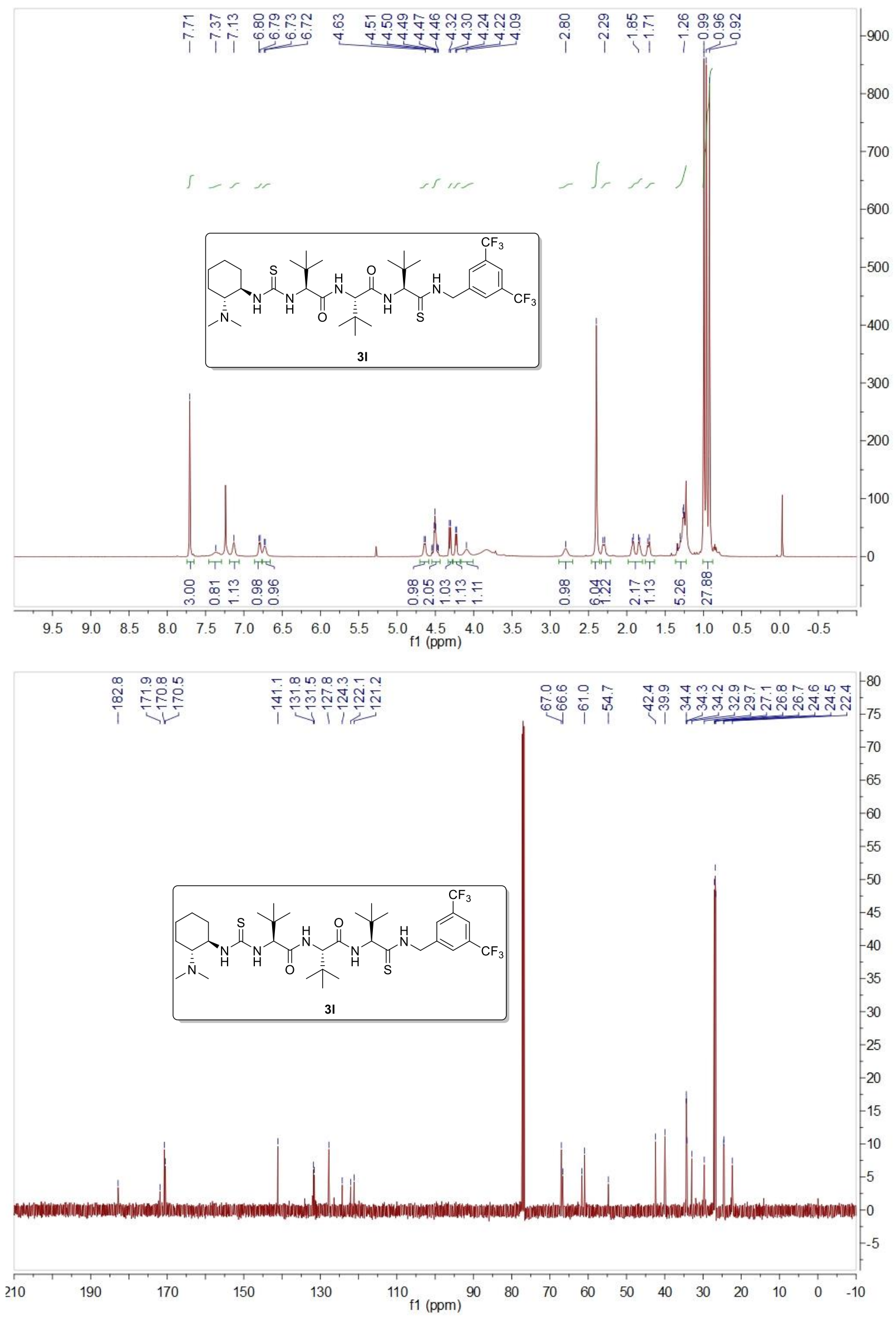

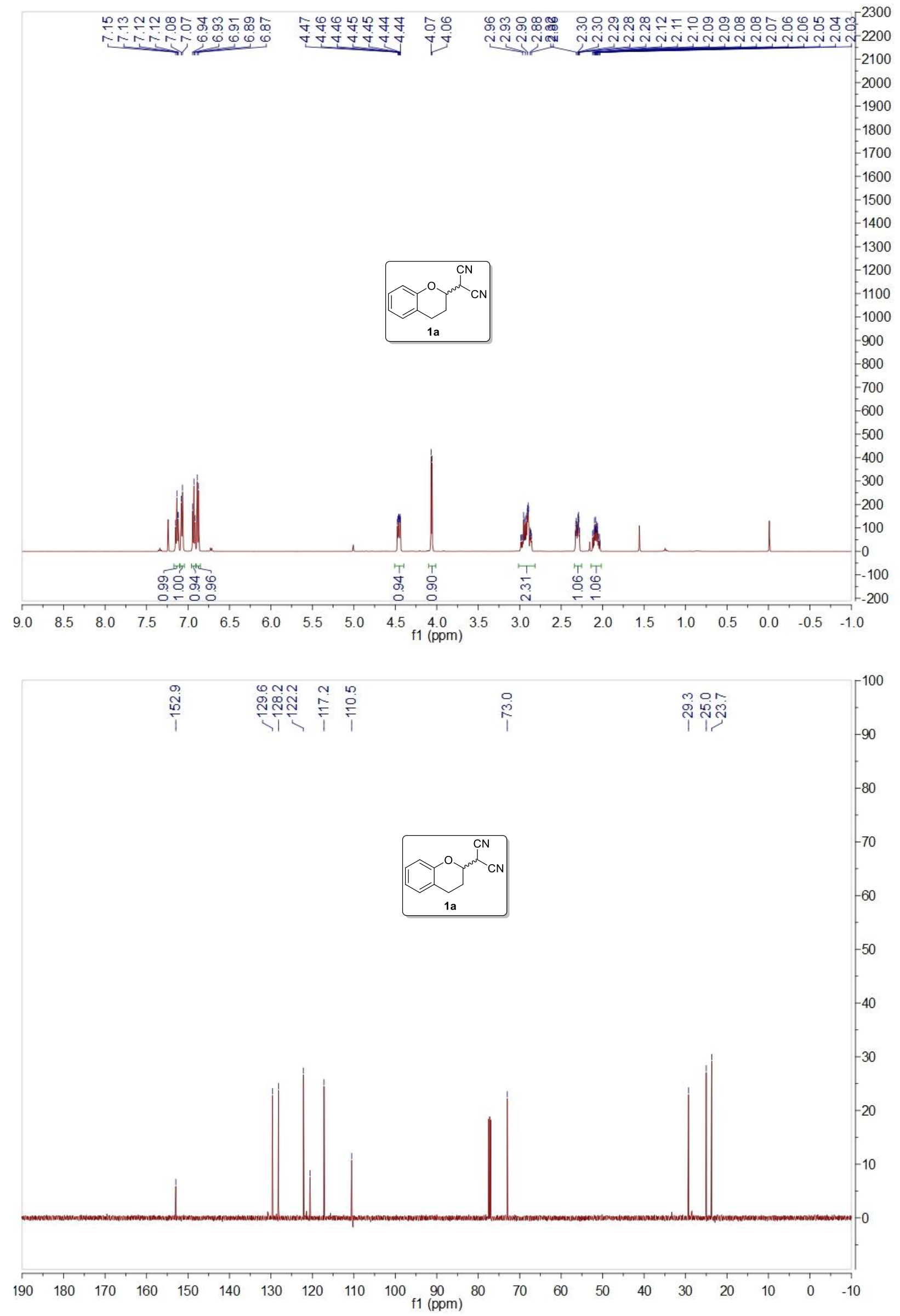

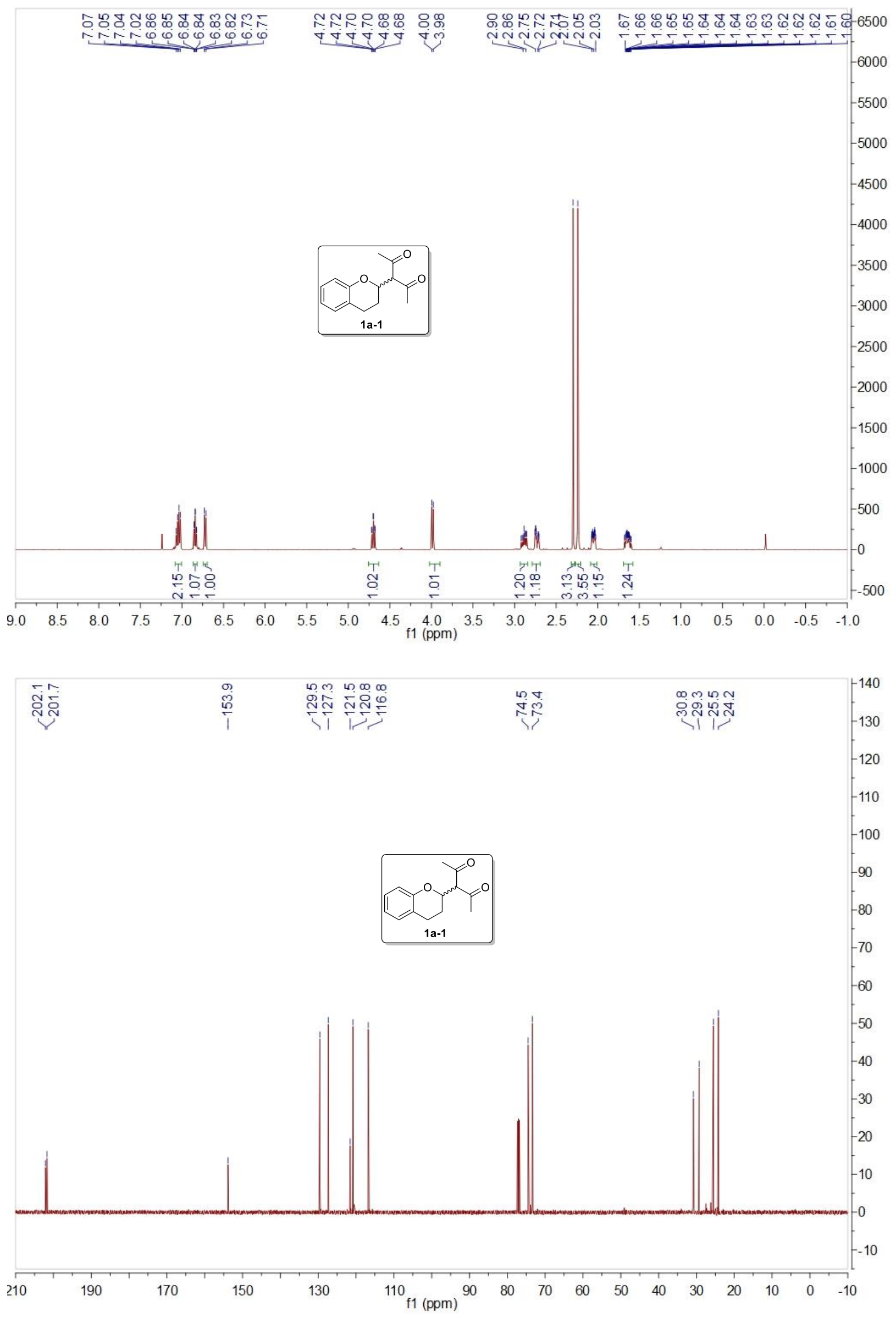

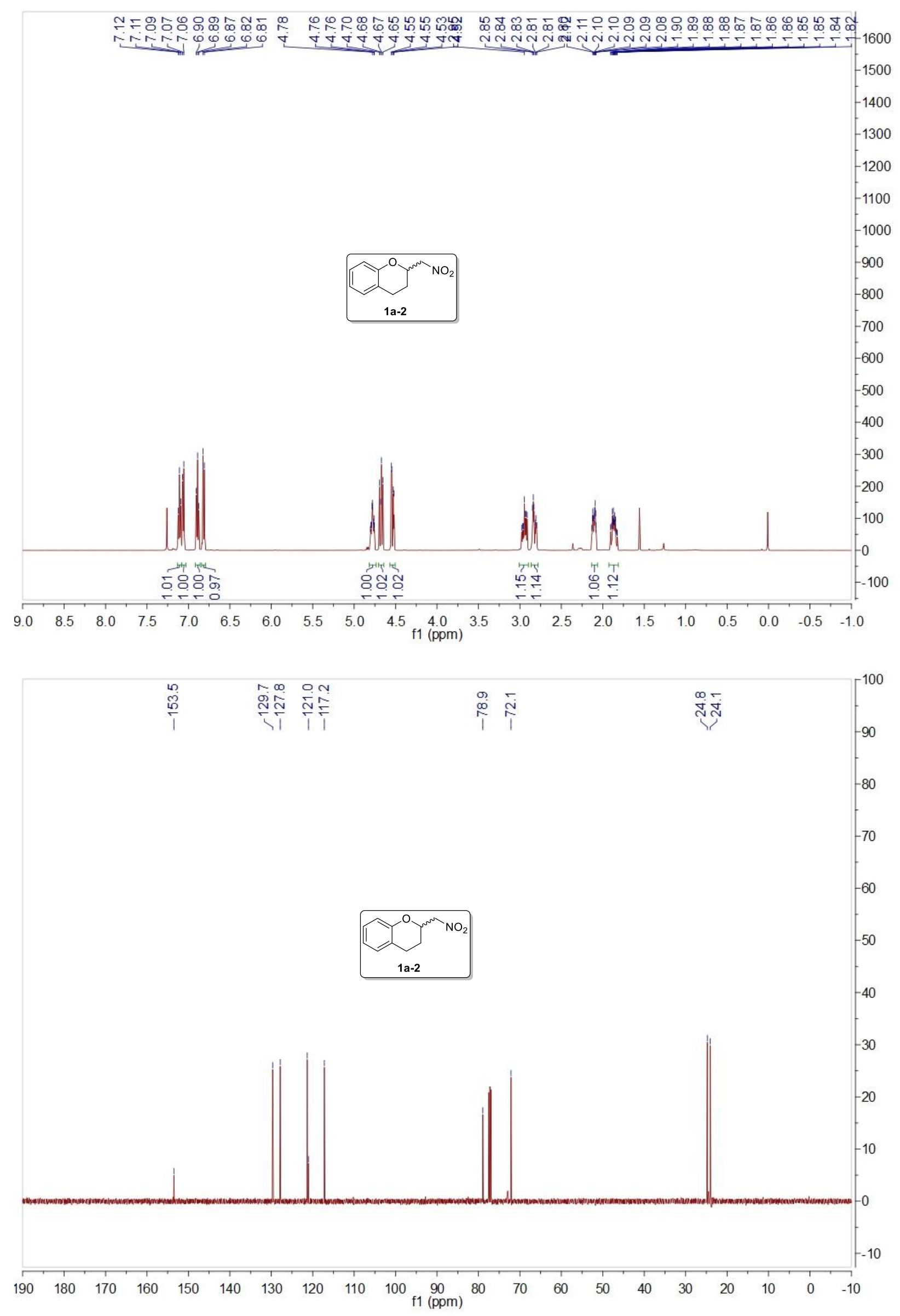


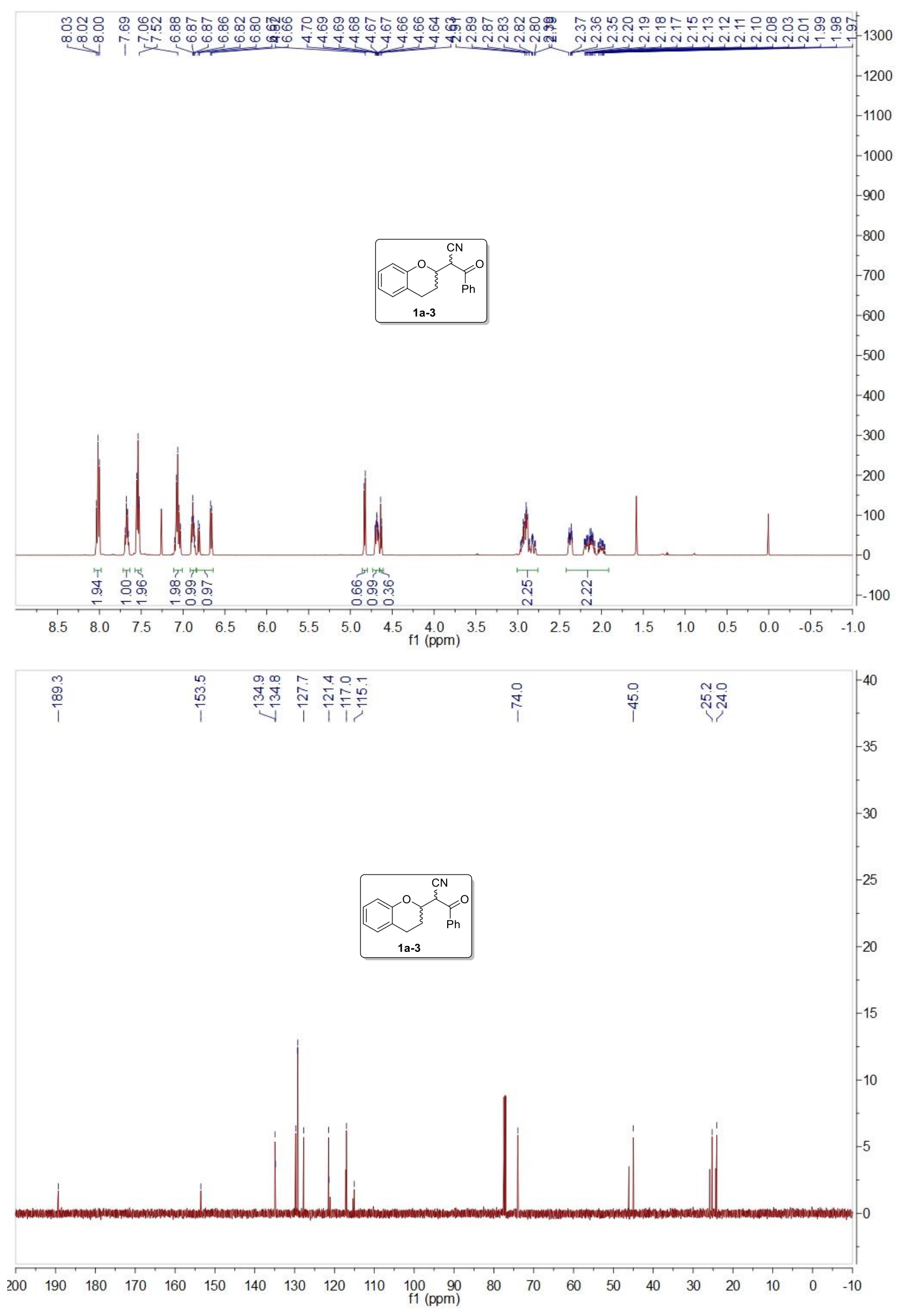



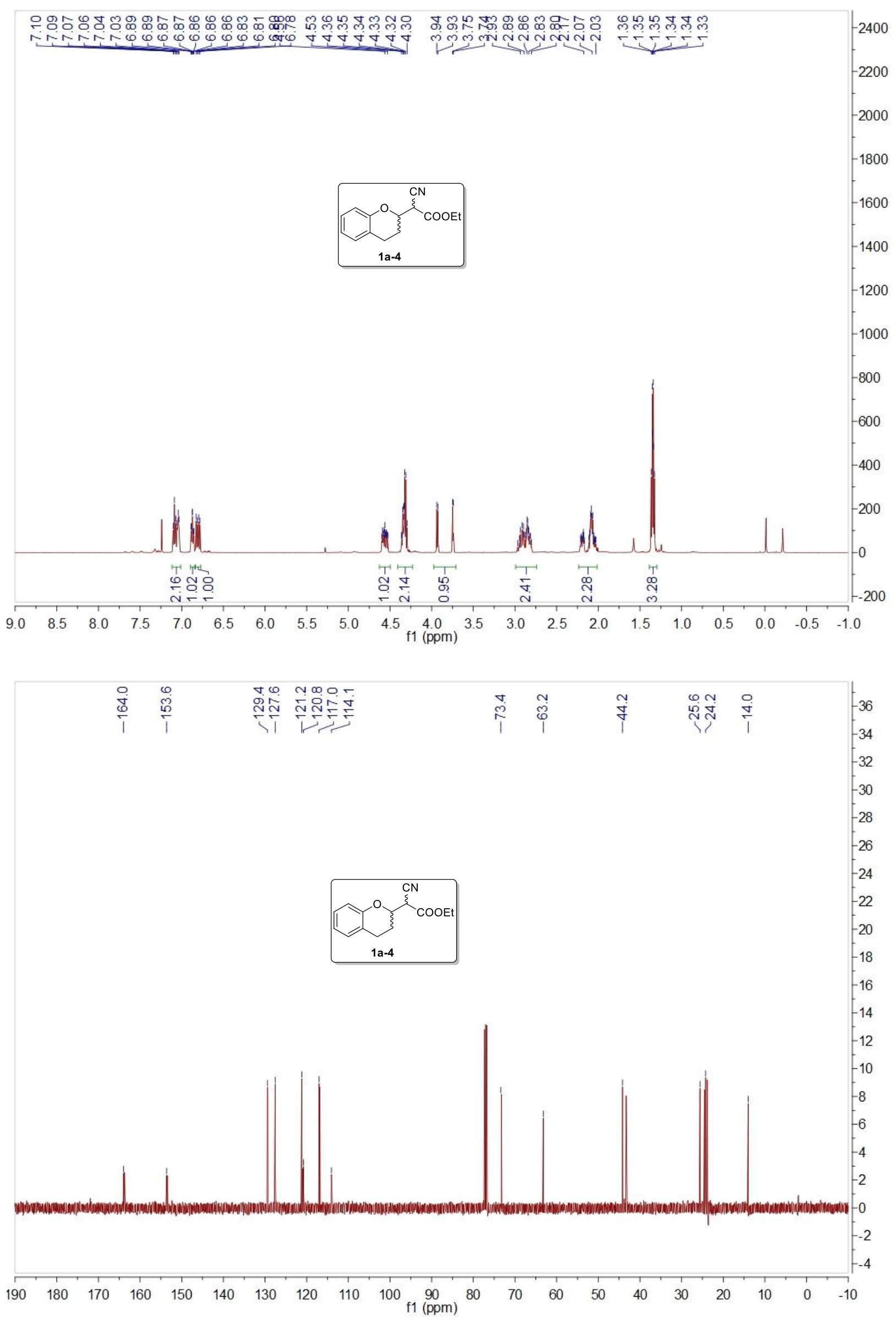

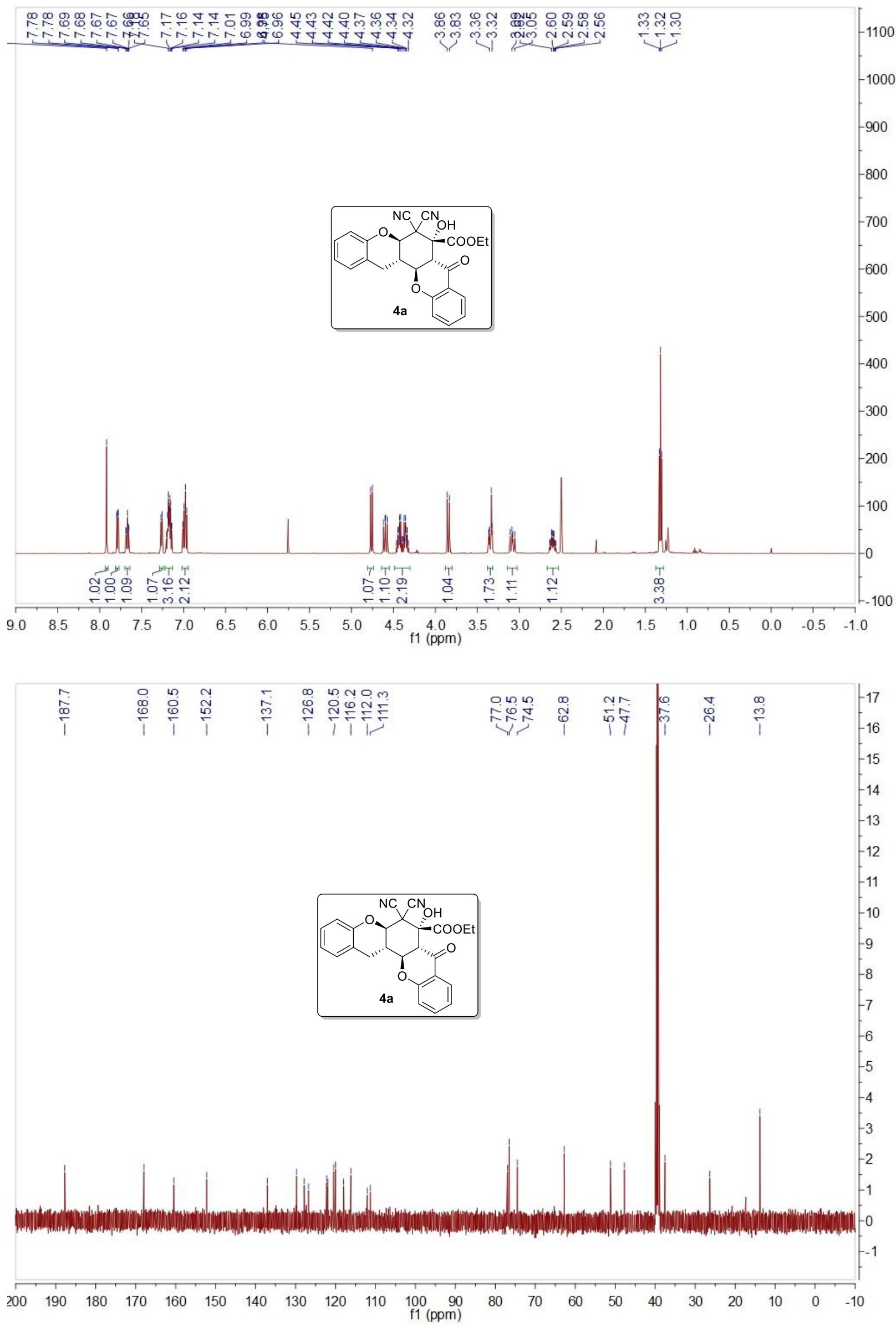
Chrom Type: Fixed WL Chromatogram, 210 nm

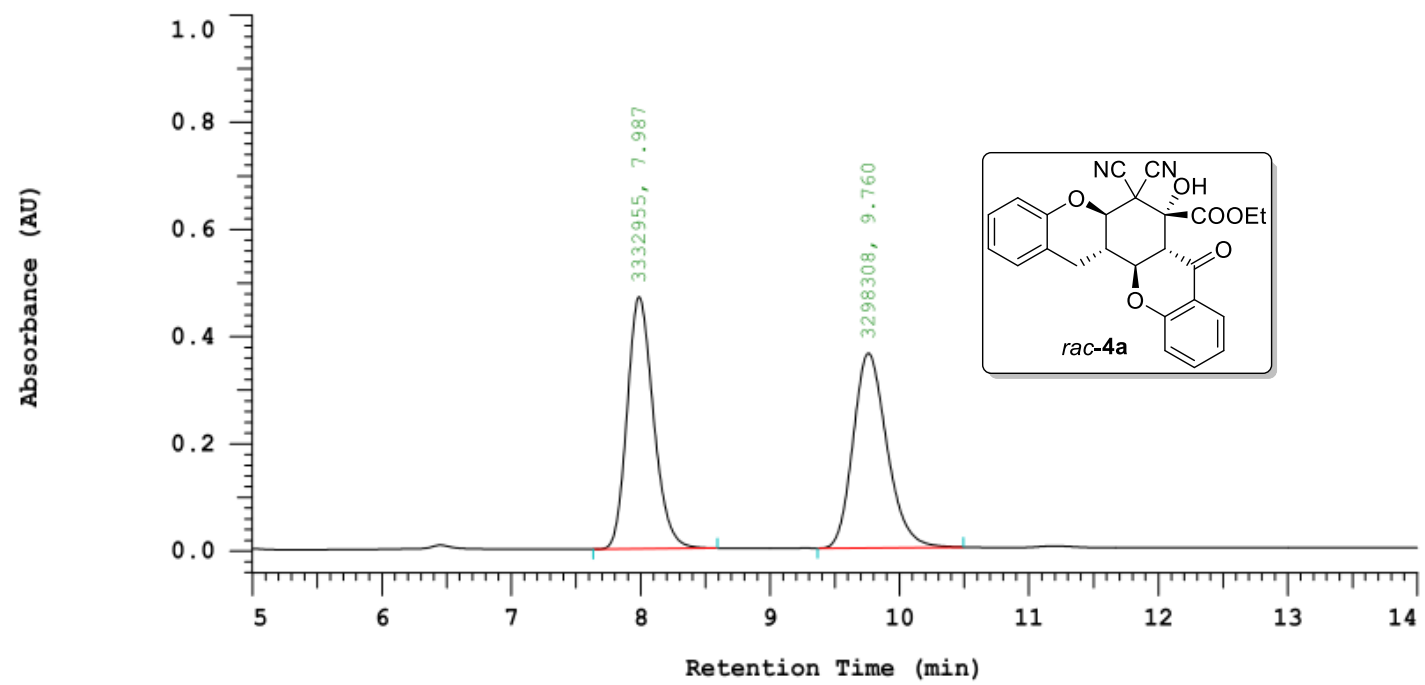

Chrom Type: Fixed WL Chromatogram, $210 \mathrm{~nm}$

Peak Quantitation: AREA

Calculation Method: AREA응

\begin{tabular}{ccccc} 
No. & RT & Area & Area & BC \\
\hline 1 & 7.987 & 3332955 & 50.261 & BB \\
2 & 9.760 & 3298308 & 49.739 & BB \\
\hline & & 6631263 & 100.000 & \\
\hline
\end{tabular}

Chrom Type: Fixed WL Chromatogram, $210 \mathrm{~nm}$

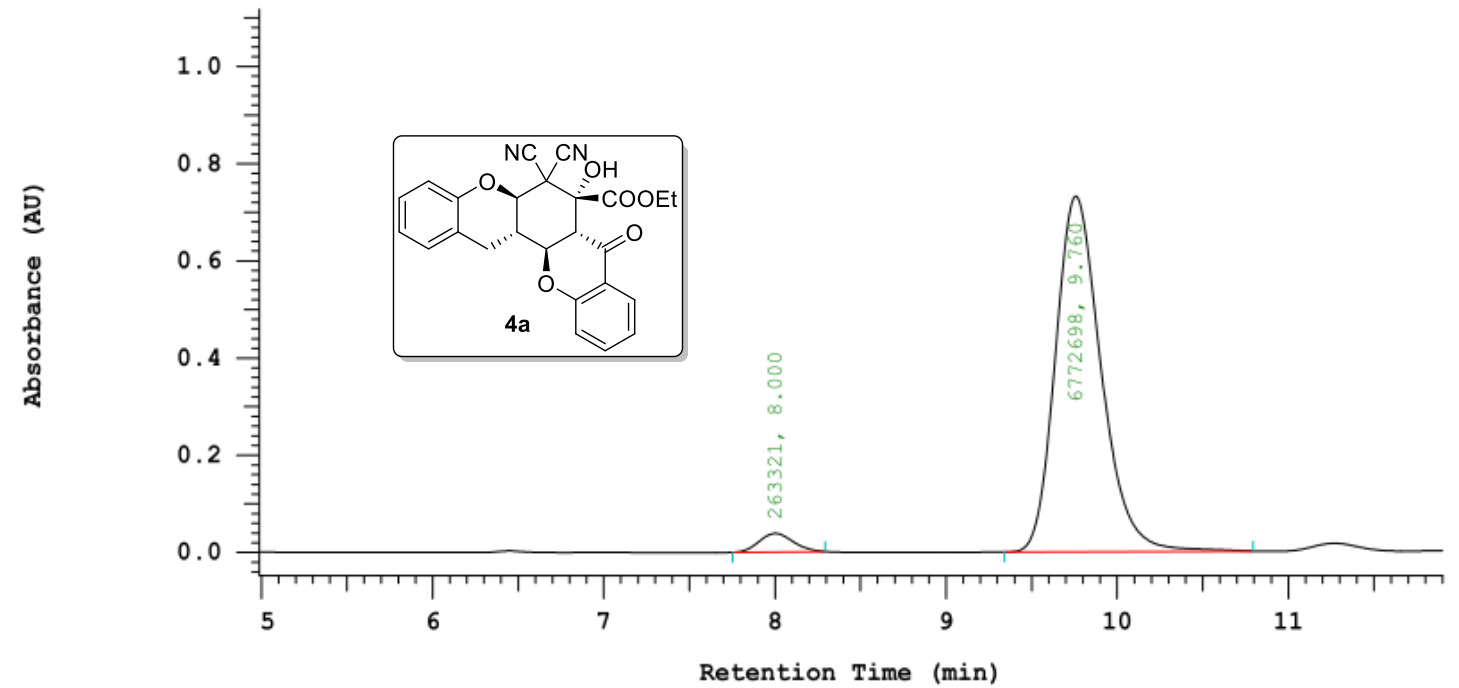

Chrom Type: Fixed WL Chromatogram, $210 \mathrm{~nm}$

Peak Quantitation: AREA

Calculation Method: AREA\%

\begin{tabular}{|c|c|c|c|c|}
\hline No. & RT & Area & Area 응 & $\mathrm{BC}$ \\
\hline 1 & 8.000 & 263321 & 3.742 & $\mathrm{BB}$ \\
\hline 2 & 9.760 & 6772698 & 96.258 & BB \\
\hline & & 7036019 & 100.000 & \\
\hline
\end{tabular}



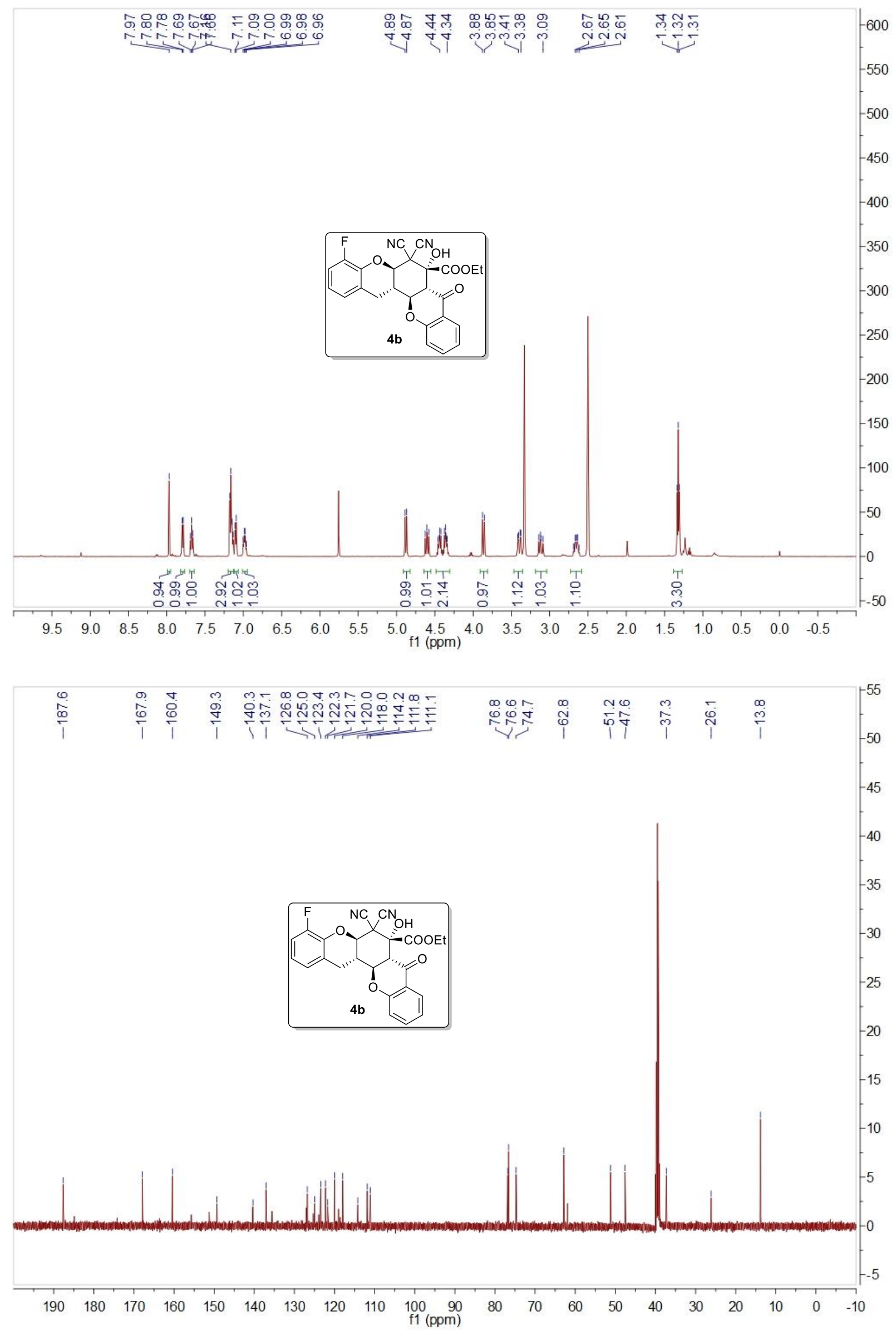
Chrom Type: Fixed WL Chromatogram, $210 \mathrm{~nm}$

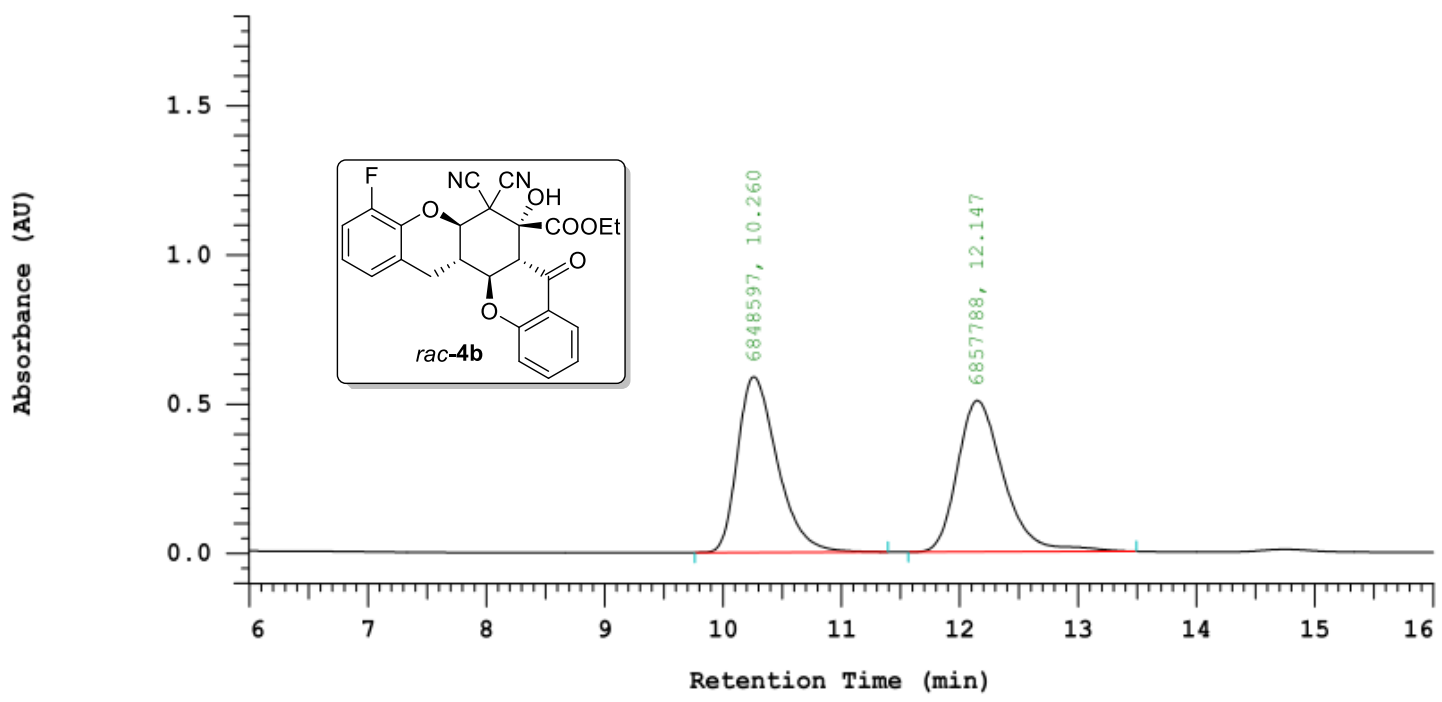

Chrom Type: Fixed WL Chromatogram, $210 \mathrm{~nm}$

Peak Quantitation: AREA

Calculation Method: AREA응

\begin{tabular}{ccccc} 
No. & RT & Area & Area & BC \\
\hline 1 & 10.260 & 6848597 & 49.966 & BB \\
2 & 12.147 & 6857788 & 50.034 & BB \\
\hline & & 13706385 & 100.000 & \\
\hline
\end{tabular}

Chrom Type: Fixed WL Chromatogram, 210 nm

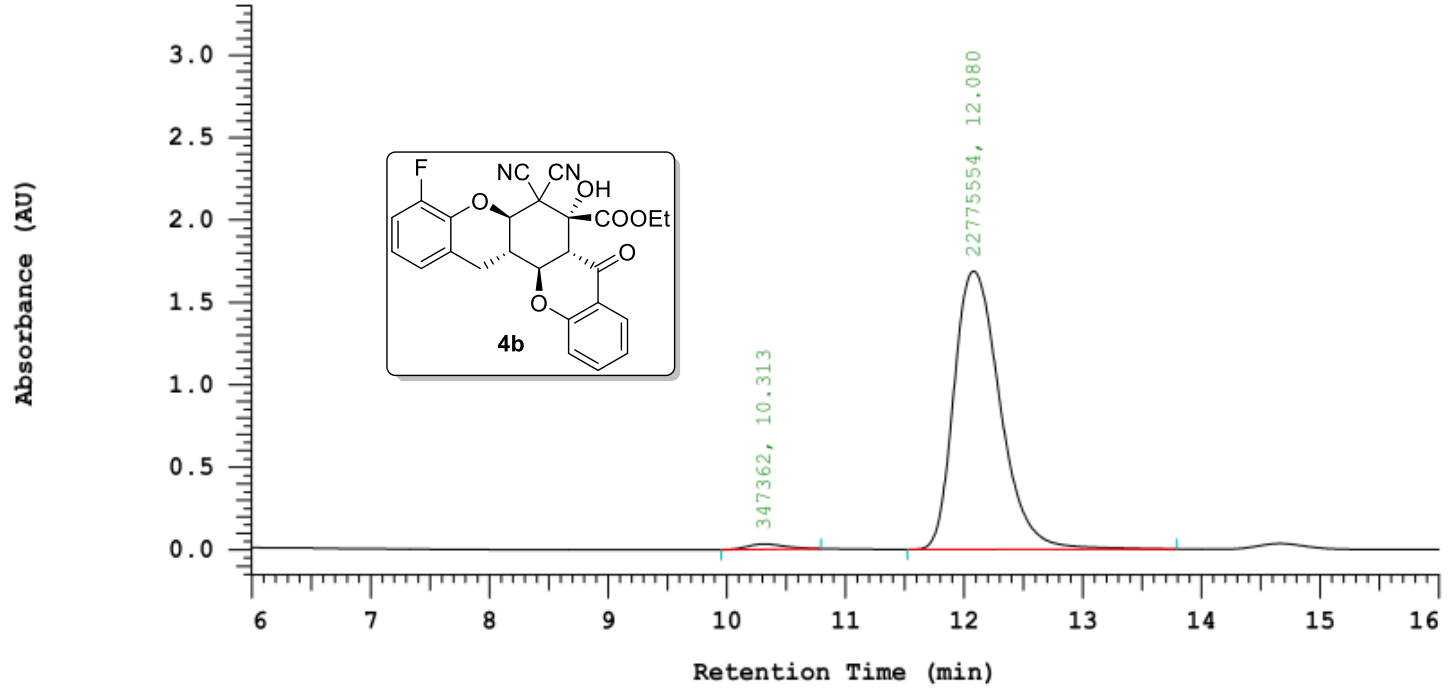

Chrom Type: Fixed WL Chromatogram, $210 \mathrm{~nm}$

Peak Quantitation: AREA

Calculation Method: AREA\%

\begin{tabular}{|c|c|c|c|c|}
\hline No. & $\mathrm{RT}$ & Area & Area 웅 & $\mathrm{BC}$ \\
\hline 1 & 10.313 & 347362 & 1.502 & BB \\
\hline 2 & 12.080 & 22775554 & 98.498 & $\mathrm{BB}$ \\
\hline & & 23122916 & 100.000 & \\
\hline
\end{tabular}



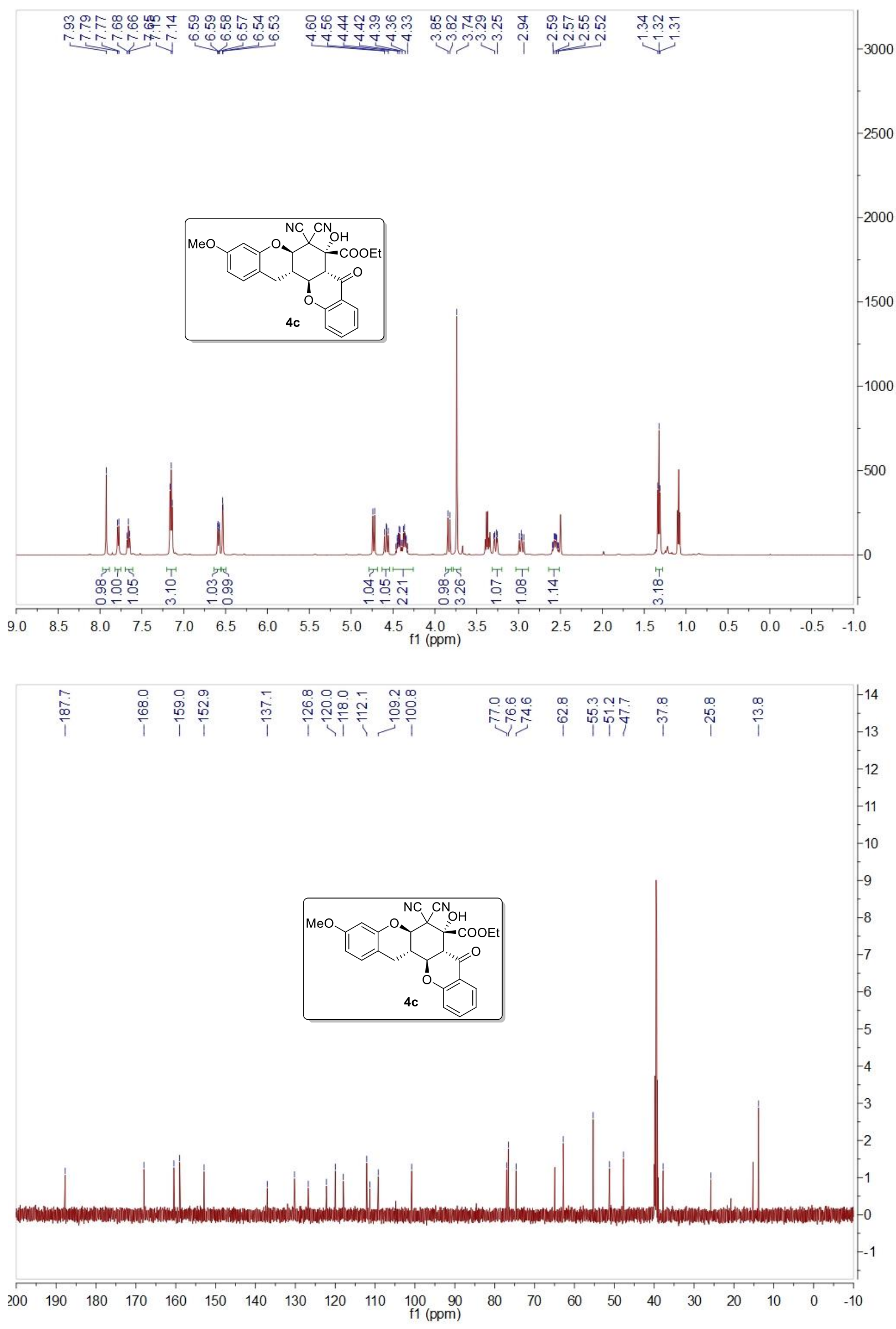
Chrom Type: Fixed WL Chromatogram, $210 \mathrm{~nm}$

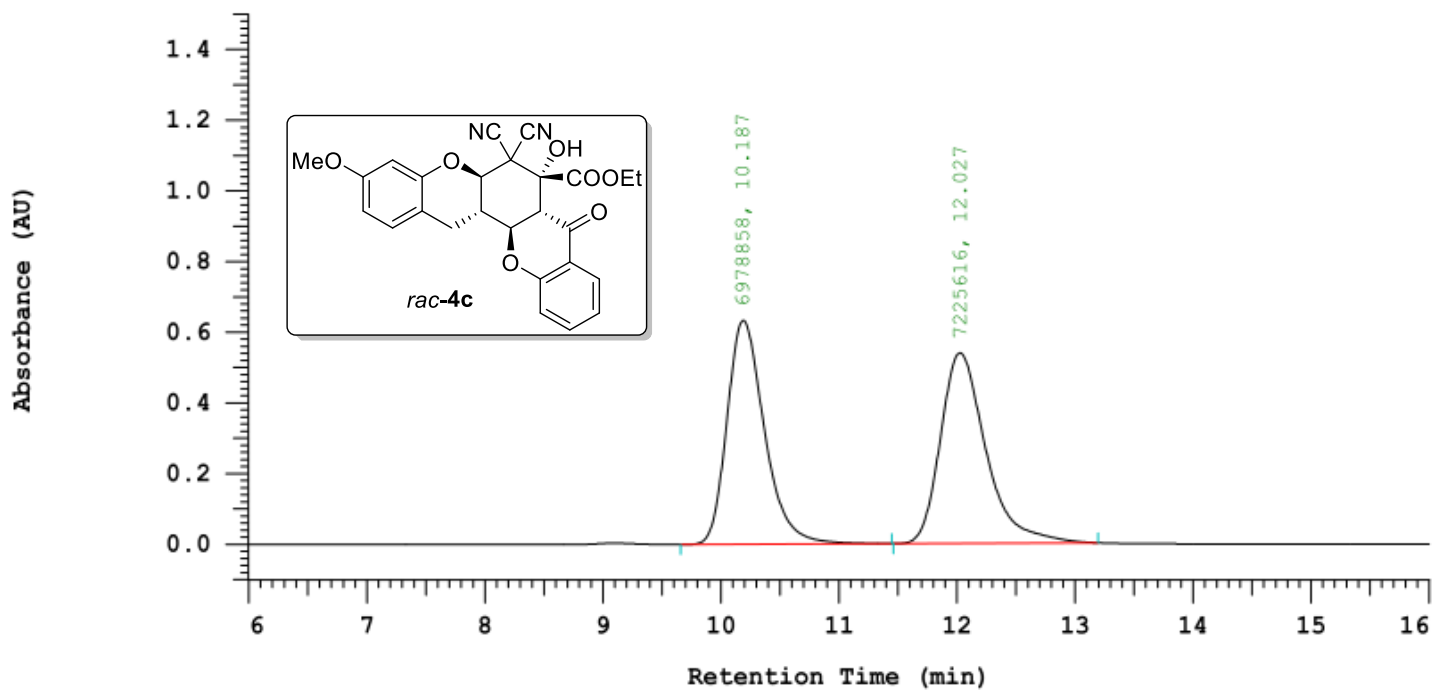

Chrom Type: Fixed WL Chromatogram, $210 \mathrm{~nm}$

Peak Quantitation: AREA

Calculation Method: AREA음

\begin{tabular}{ccccc} 
No. & RT & Area & Area & BC \\
\hline 1 & 10.187 & 6978858 & 49.131 & BB \\
2 & 12.027 & 7225616 & 50.869 & BB \\
\hline & & 14204474 & 100.000 & \\
\hline
\end{tabular}

Chrom Type: Fixed WL Chromatogram, $210 \mathrm{~nm}$

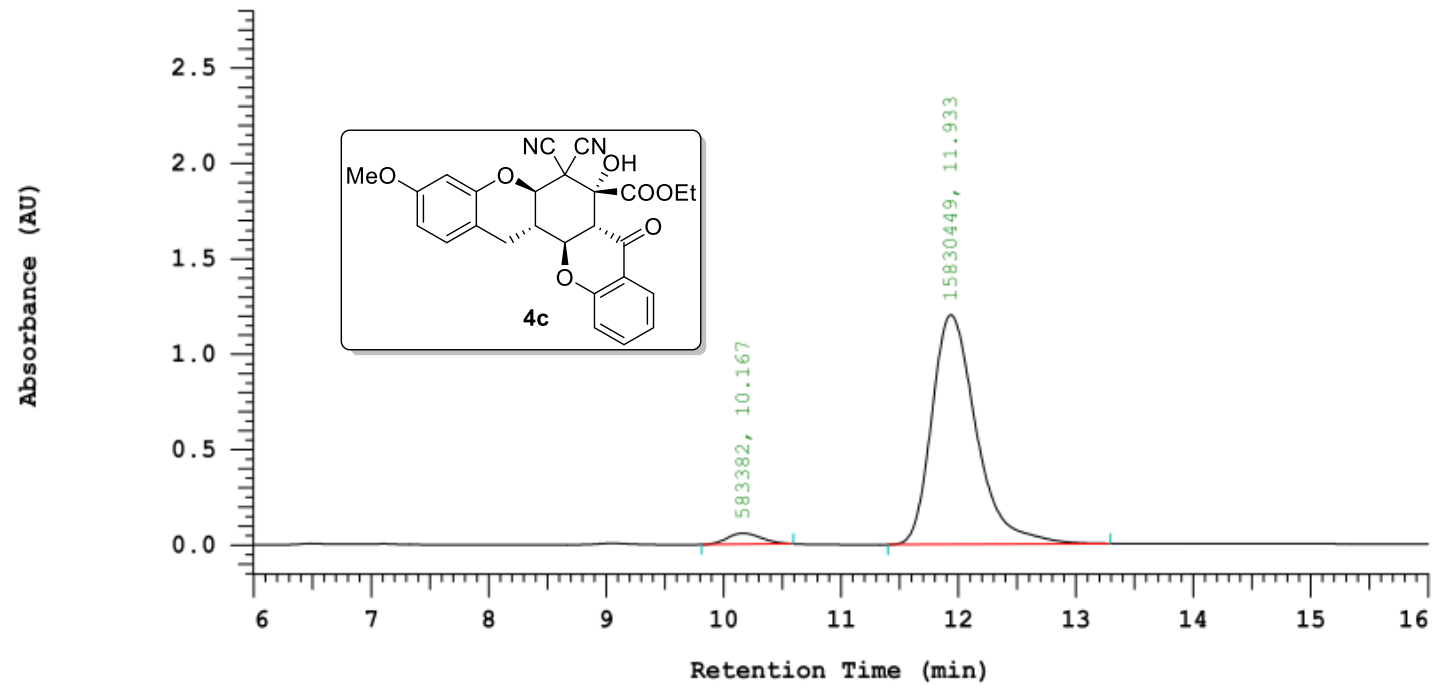

Chrom Type: Fixed WL Chromatogram, $210 \mathrm{~nm}$

Peak Quantitation: AREA

Calculation Method: AREA\%

\begin{tabular}{|c|c|c|c|c|}
\hline No. & RT & Area & Area \% & $\mathrm{BC}$ \\
\hline 1 & 10.167 & 583382 & 3.554 & $\mathrm{BB}$ \\
\hline \multirow[t]{2}{*}{2} & 11.933 & 15830449 & 96.446 & $\mathrm{BB}$ \\
\hline & & 16413831 & 100.000 & \\
\hline
\end{tabular}



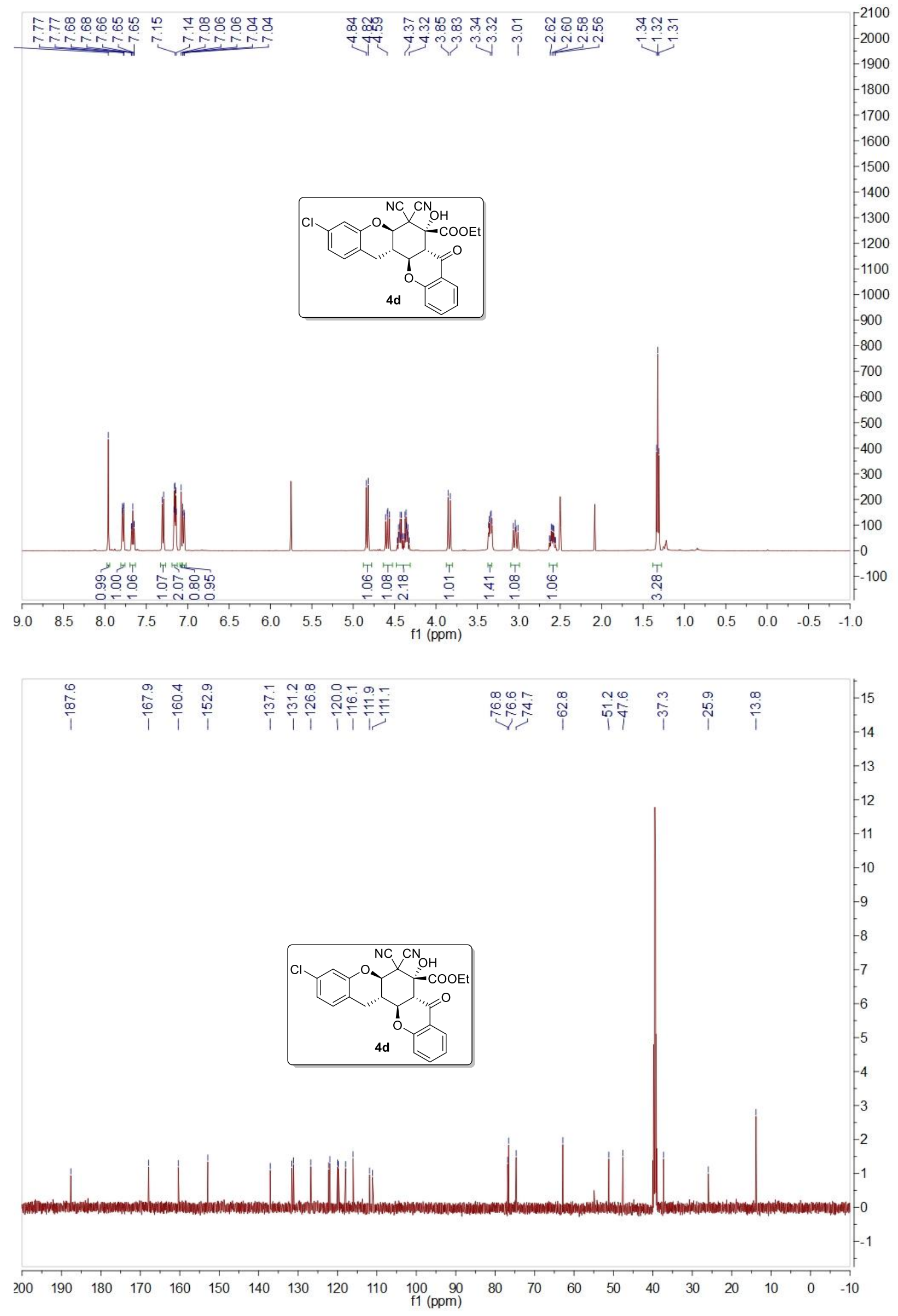
Chrom Type: Fixed WL Chromatogram, $210 \mathrm{~nm}$

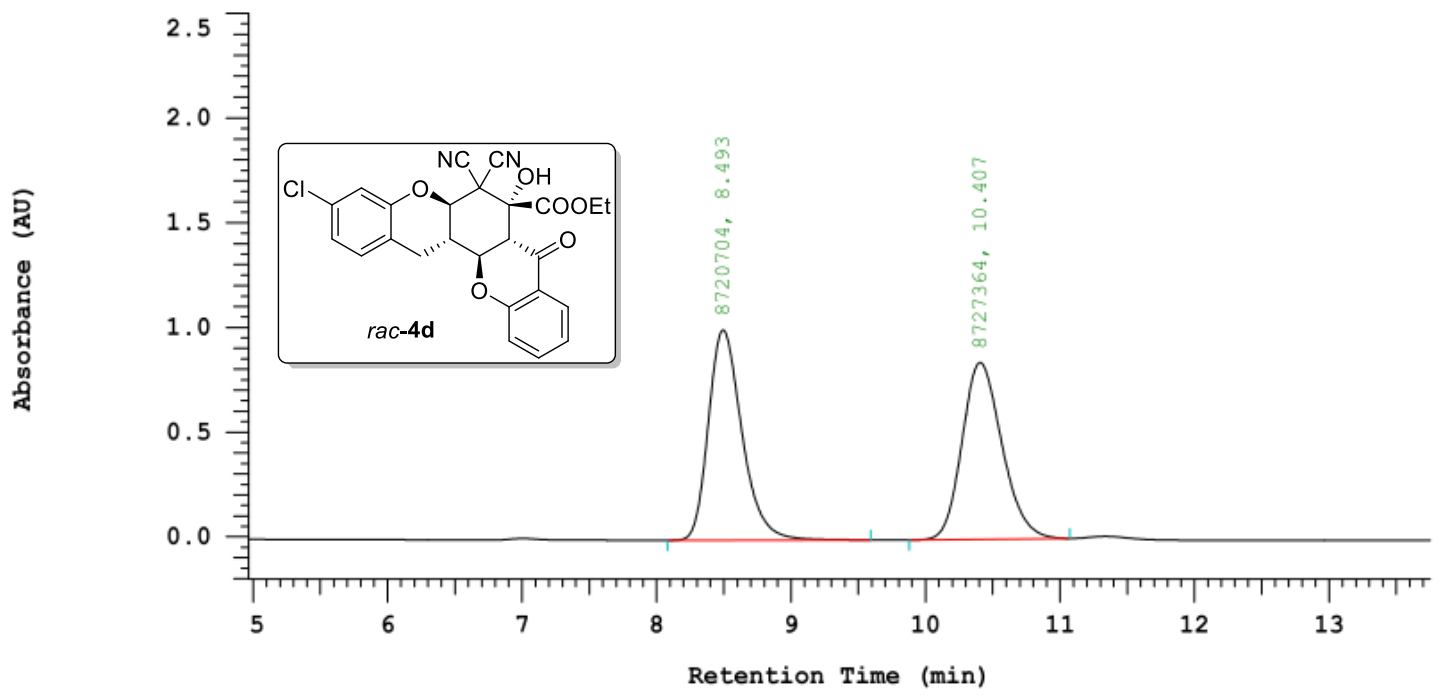

Chrom Type: Fixed WL Chromatogram, $210 \mathrm{~nm}$

Peak Quantitation: AREA

Calculation Method: AREA응

\begin{tabular}{|c|c|c|c|c|}
\hline No. & $\mathrm{RT}$ & Area & Area $\%$ & $\mathrm{BC}$ \\
\hline 1 & 8.493 & 8720704 & 49.981 & $\mathrm{BB}$ \\
\hline 2 & 10.407 & 8727364 & 50.019 & $\mathrm{BB}$ \\
\hline & & 17448068 & 100.000 & \\
\hline
\end{tabular}

Chrom Type: Fixed WL Chromatogram, 210 nm

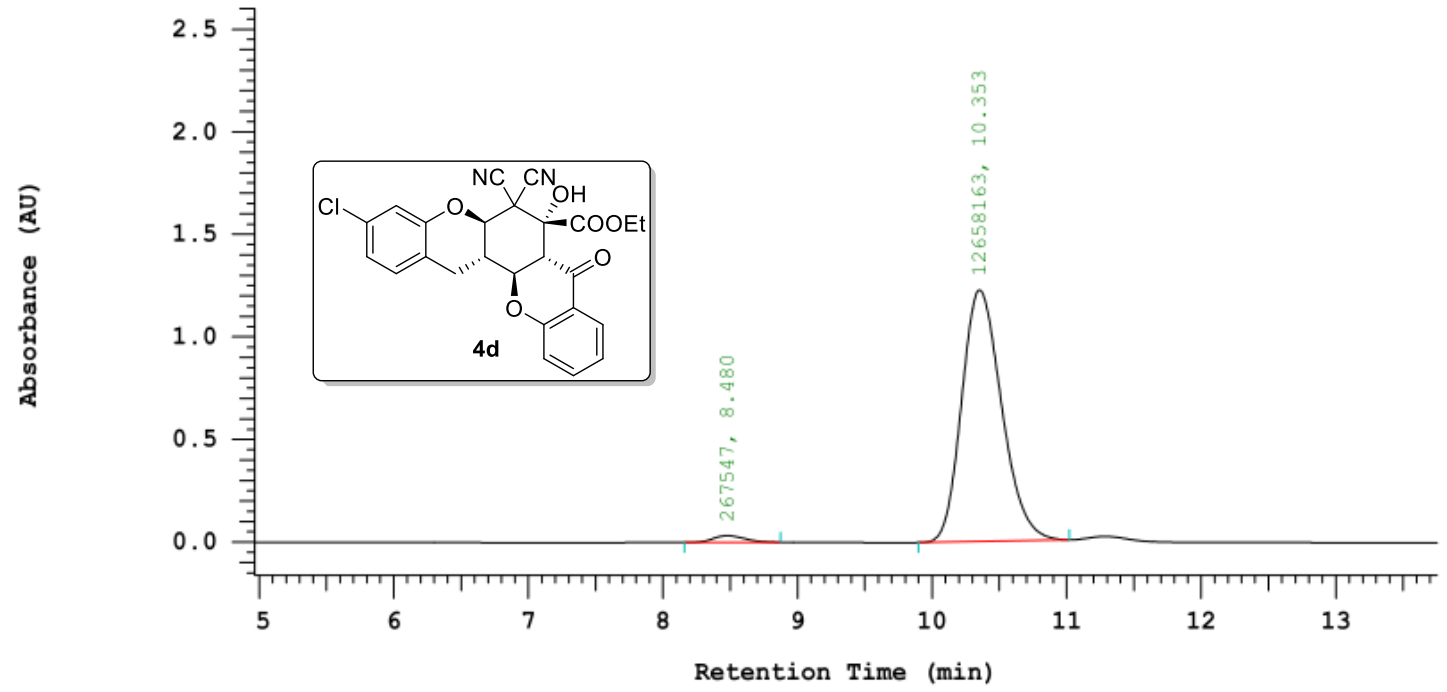

Chrom Type: Fixed WL Chromatogram, $210 \mathrm{~nm}$

Peak Quantitation: AREA

Calculation Method: AREA응

\begin{tabular}{|c|c|c|c|c|}
\hline No. & $\mathrm{RT}$ & Area & Area \% & $\mathrm{BC}$ \\
\hline 1 & 8.480 & 267547 & 2.070 & BB \\
\hline 2 & 10.353 & 12658163 & 97.930 & $\mathrm{BB}$ \\
\hline & & 12925710 & 100.000 & \\
\hline
\end{tabular}



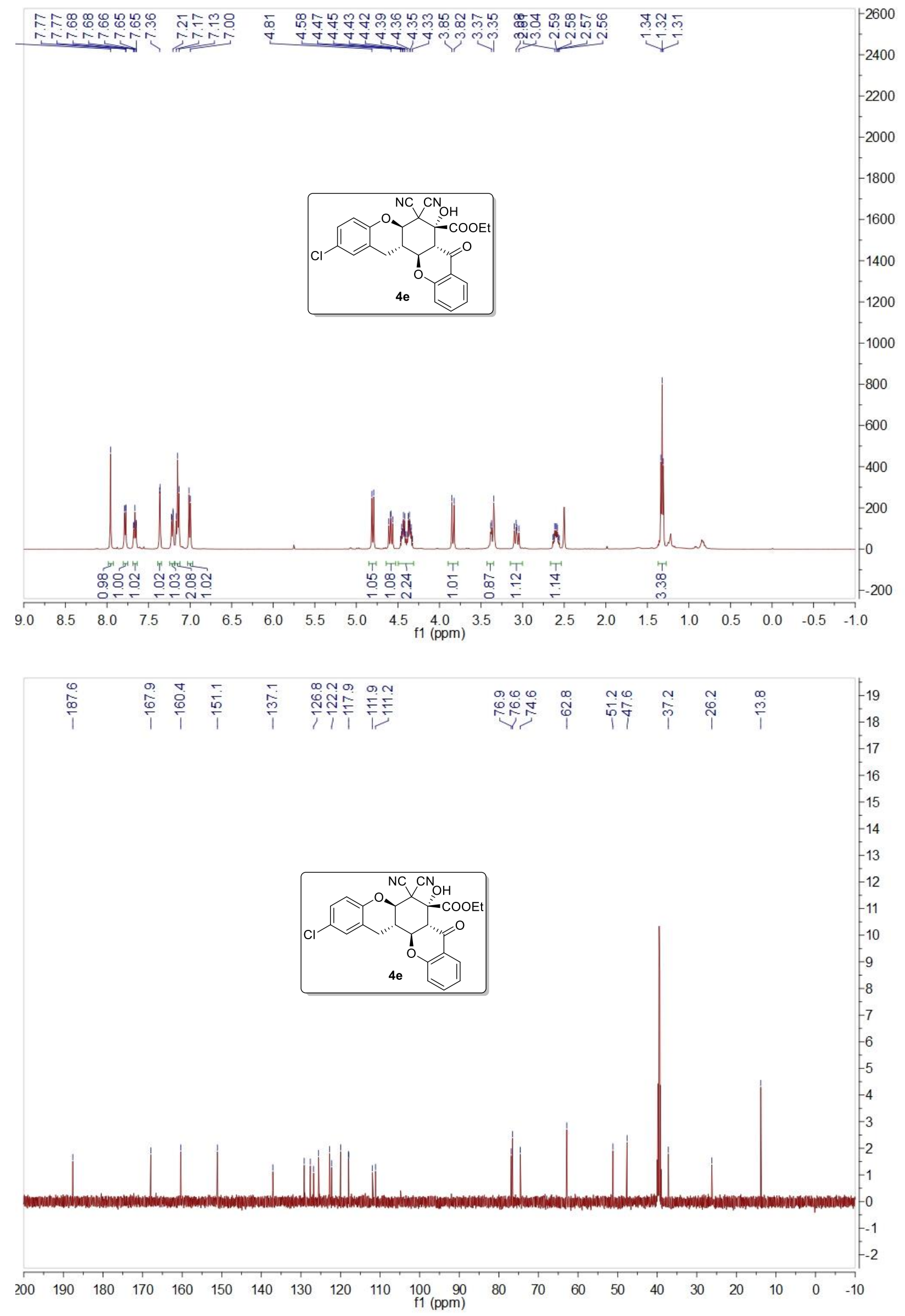
Chrom Type: Fixed WL Chromatogram, $210 \mathrm{~nm}$

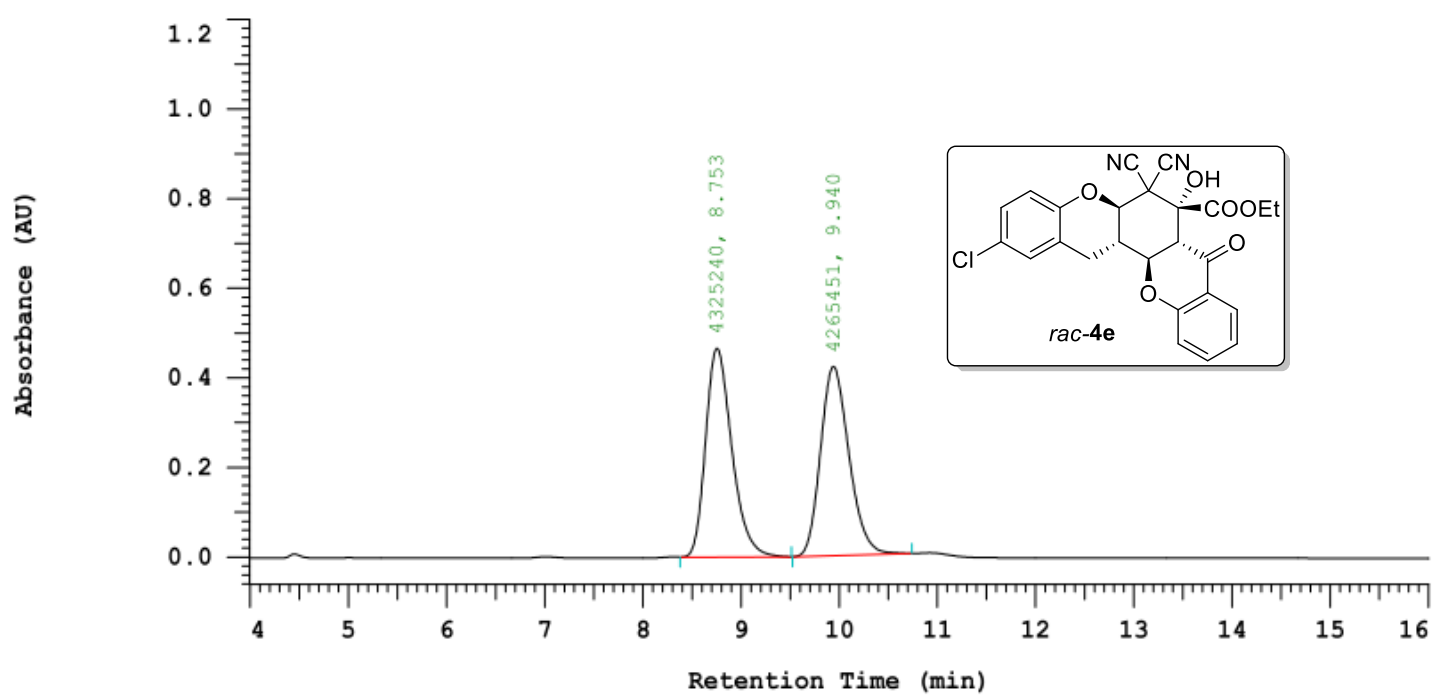

Chrom Type: Fixed WL Chromatogram, $210 \mathrm{~nm}$

Peak Quantitation: AREA

Calculation Method: AREA\%

\begin{tabular}{ccccc} 
No. & RT & Area & Area $\frac{\text { o }}{5}$ & BC \\
\hline 1 & 8.753 & 4325240 & 50.348 & BB \\
2 & 9.940 & 4265451 & 49.652 & BB \\
\hline & & 8590691 & 100.000 & \\
\hline
\end{tabular}

Chrom Type: Fixed WL Chromatogram, $210 \mathrm{~nm}$

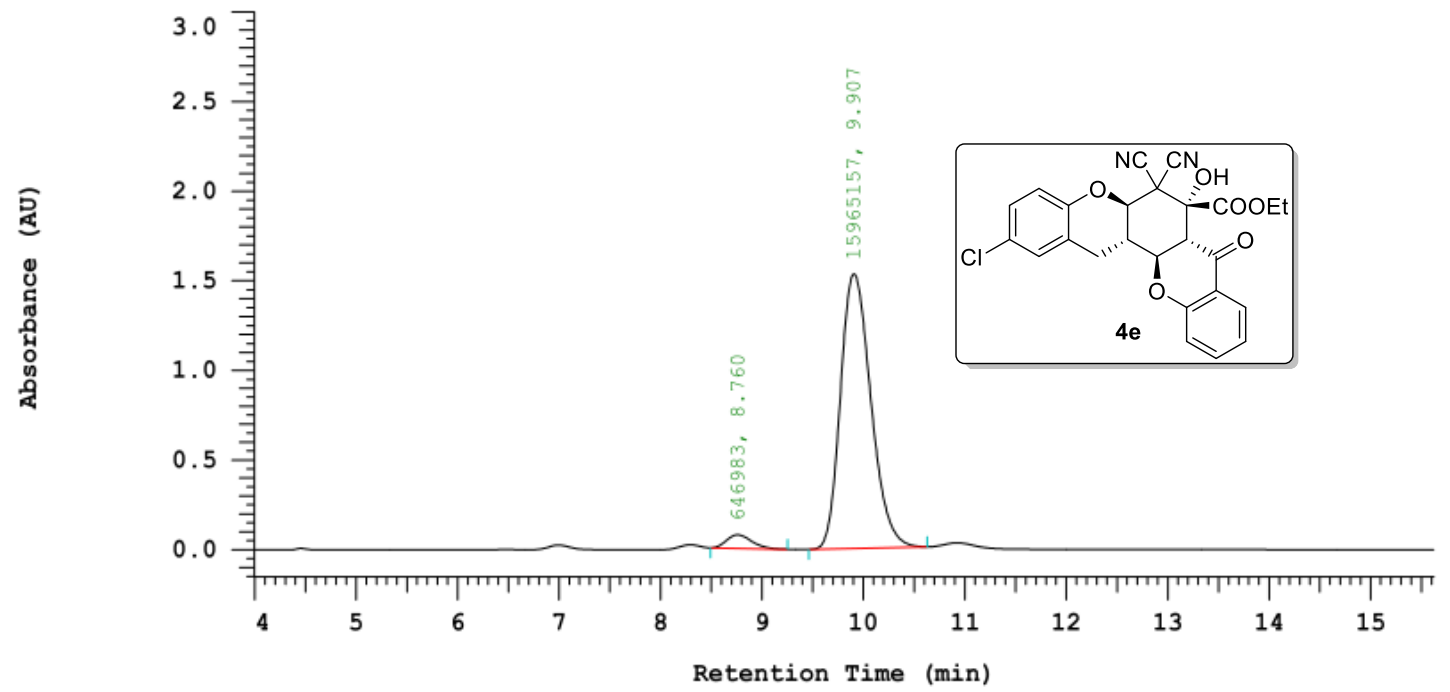

Chrom Type: Fixed WL Chromatogram, $210 \mathrm{~nm}$

Peak Quantitation: AREA

Calculation Method: AREA

\begin{tabular}{|c|c|c|c|c|}
\hline No. & $\mathrm{RT}$ & Area & Area \% & $\mathrm{BC}$ \\
\hline 1 & 8.760 & 646983 & 3.895 & $\mathrm{BB}$ \\
\hline \multirow[t]{2}{*}{2} & 9.907 & 15965157 & 96.105 & $\mathrm{BB}$ \\
\hline & & 16612140 & 100.000 & \\
\hline
\end{tabular}



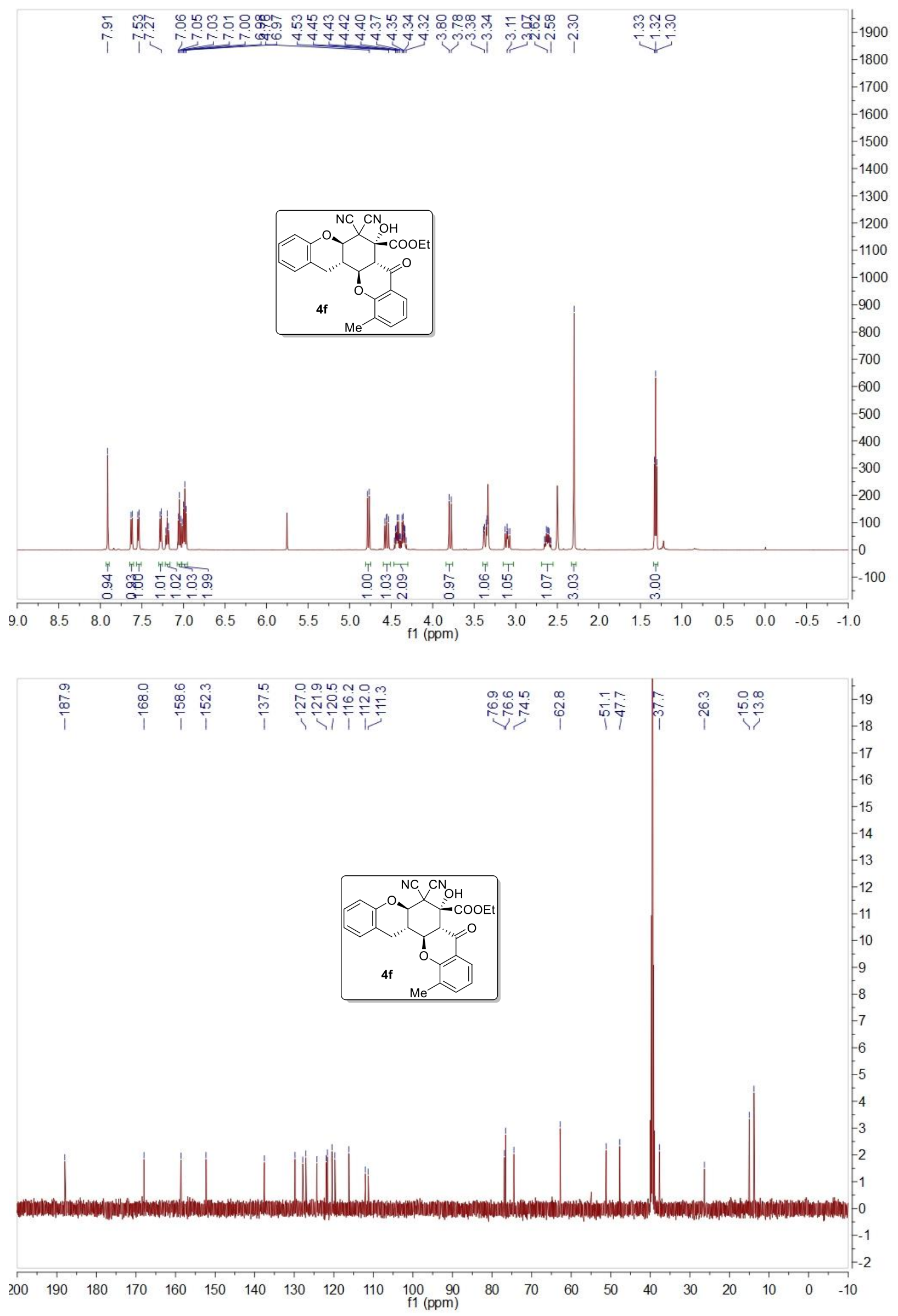


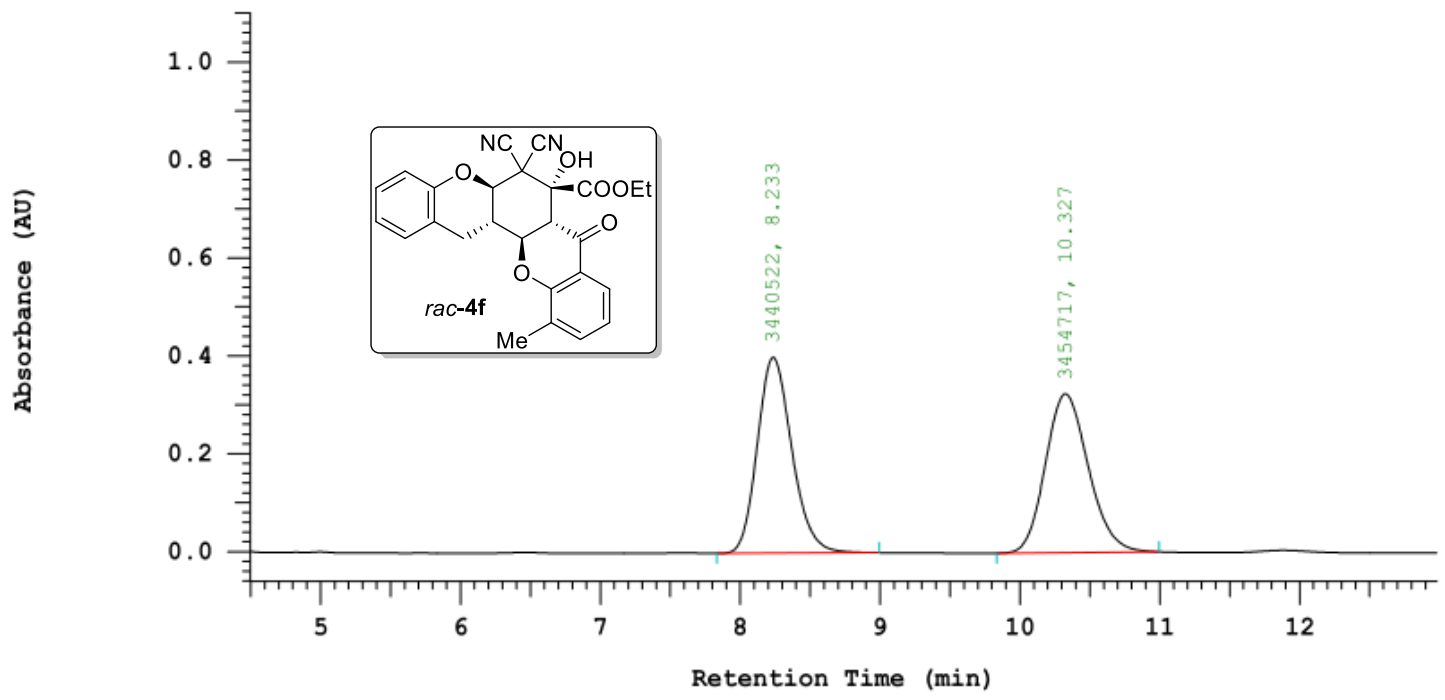

Chrom Type: Fixed WL Chromatogram, $210 \mathrm{~nm}$

Peak Quantitation: AREA

Calculation Method: AREA\%

\begin{tabular}{|c|c|c|c|c|}
\hline No. & RT & Area & Area \% & $\mathrm{BC}$ \\
\hline 1 & 8.233 & 3440522 & 49.897 & BB \\
\hline 2 & 10.327 & 3454717 & 50.103 & $\mathrm{BB}$ \\
\hline & & 6895239 & 100.000 & \\
\hline
\end{tabular}

Chrom Type: Fixed WL Chromatogram, 210 nm

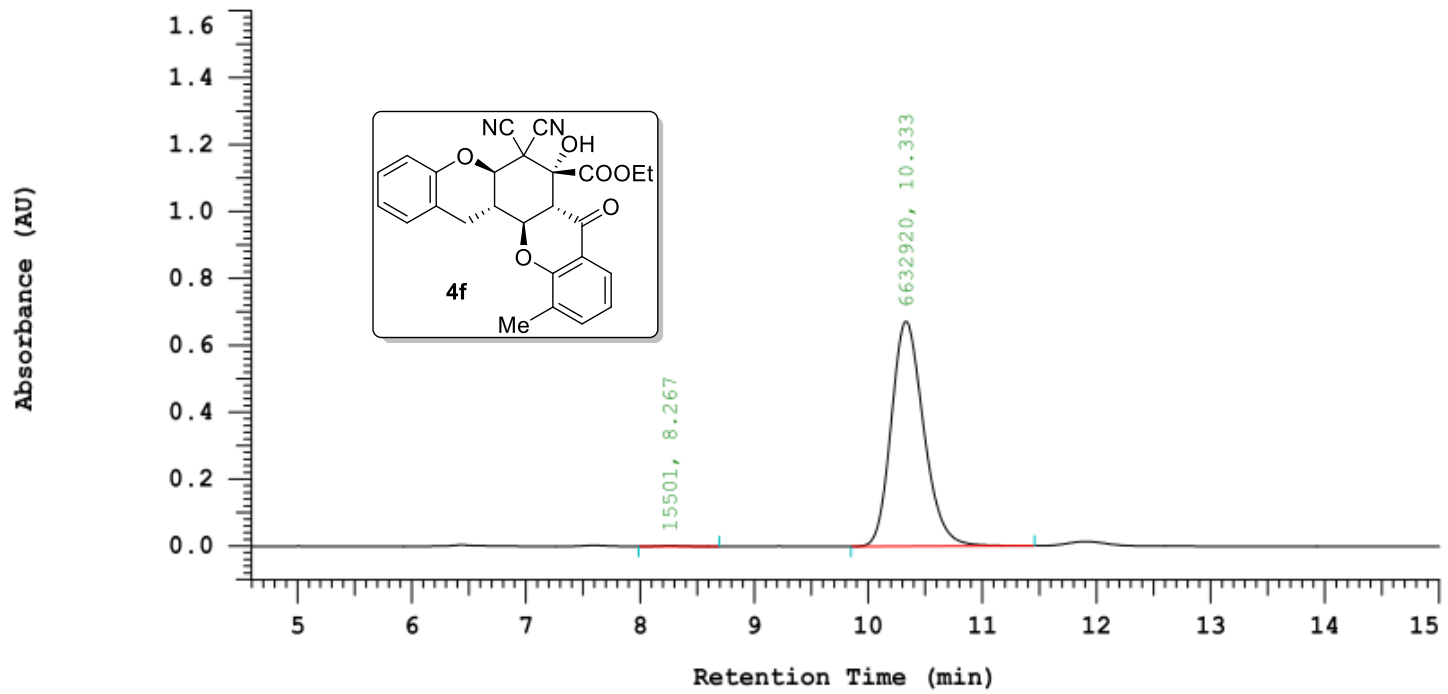

Chrom Type: Fixed WL Chromatogram, $210 \mathrm{~nm}$

Peak Quantitation: AREA

Calculation Method: AREA\%

\begin{tabular}{|c|c|c|c|c|}
\hline No. & $\mathrm{RT}$ & Area & Area \% & $\mathrm{BC}$ \\
\hline 1 & 8.267 & 15501 & 0.233 & $\mathrm{BB}$ \\
\hline \multirow[t]{2}{*}{$\begin{array}{l}1 \\
2\end{array}$} & 10.333 & 6632920 & 99.767 & $\mathrm{BB}$ \\
\hline & & 6648421 & 100.000 & \\
\hline
\end{tabular}



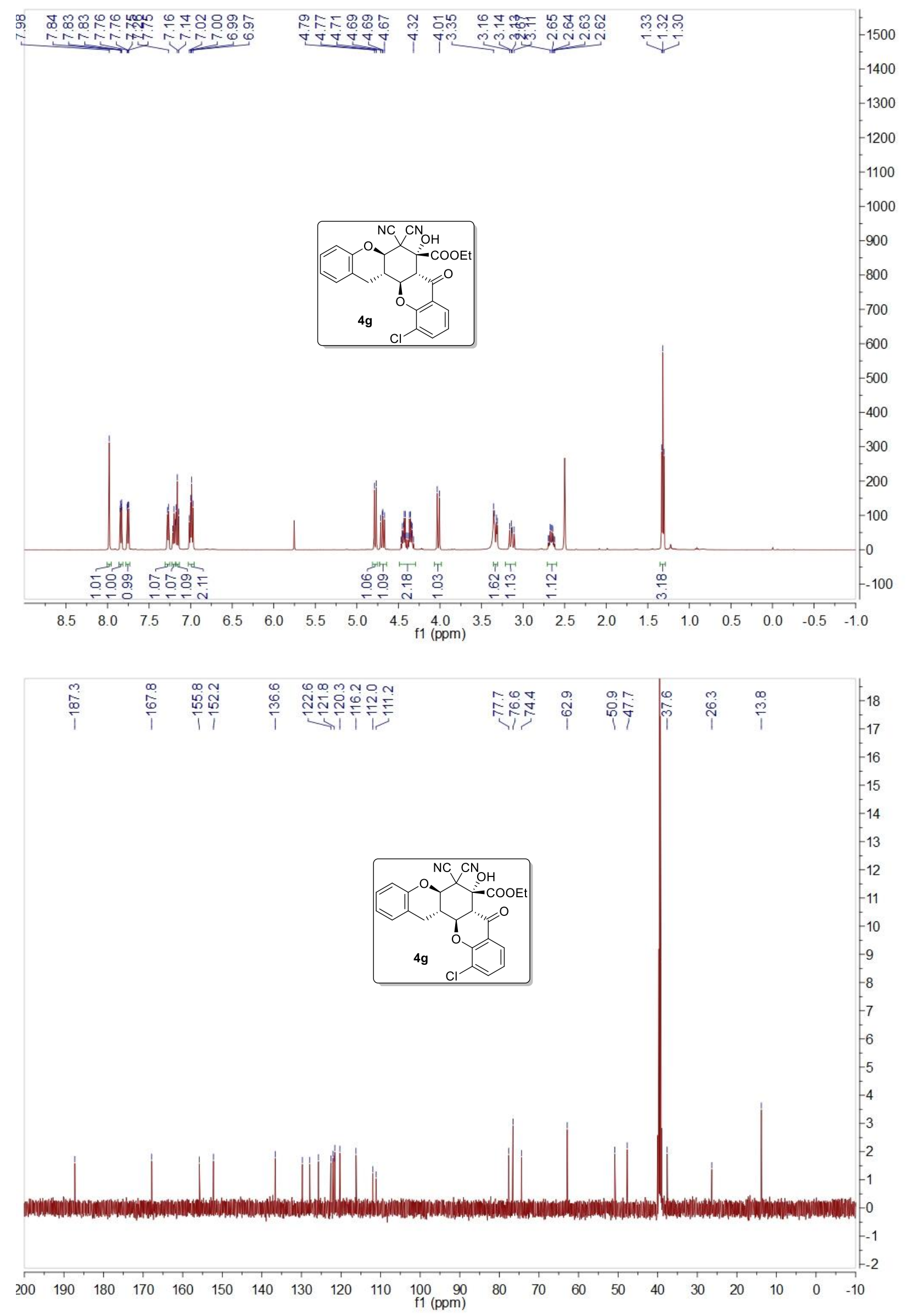
Chrom Type: Fixed WL Chromatogram, 210 nm

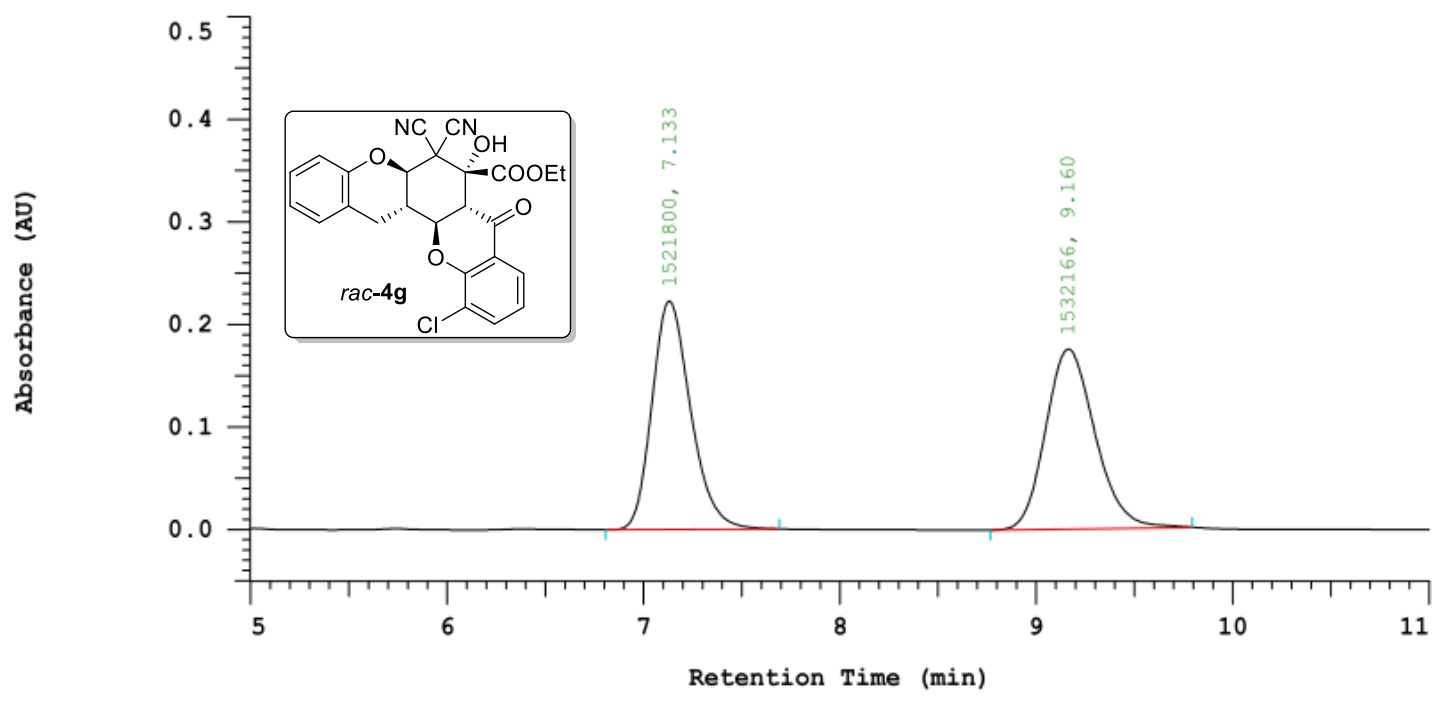

Chrom Type: Fixed WL Chromatogram, $210 \mathrm{~nm}$

Peak Quantitation: AREA

Calculation Method: AREA응

\begin{tabular}{|c|c|c|c|c|}
\hline No. & RT & Area & Area \% & $\mathrm{BC}$ \\
\hline 1 & 7.133 & 1521800 & 49.830 & $\mathrm{BB}$ \\
\hline 2 & 9.160 & 1532166 & 50.170 & BB \\
\hline & & 3053966 & 100.000 & \\
\hline
\end{tabular}

Chrom Type: Fixed WL Chromatogram, $210 \mathrm{~nm}$

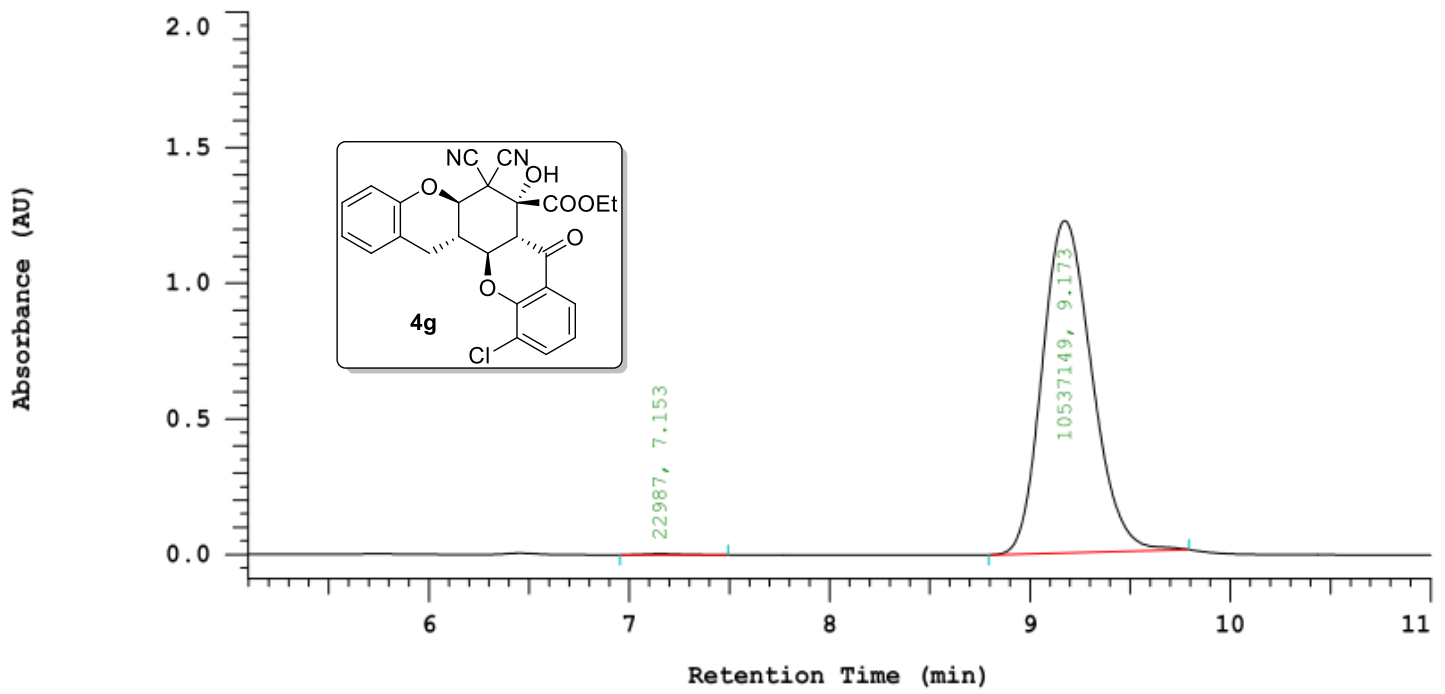

Chrom Type: Fixed WL Chromatogram, $210 \mathrm{~nm}$

Peak Quantitation: AREA

Calculation Method: AREA:

\begin{tabular}{|c|c|c|c|c|}
\hline No. & RT & Area & Area \% & $\mathrm{BC}$ \\
\hline 1 & 7.153 & 22987 & 0.218 & BB \\
\hline 2 & 9.173 & 10537149 & 99.782 & BB \\
\hline & & 10560136 & 100.000 & \\
\hline
\end{tabular}



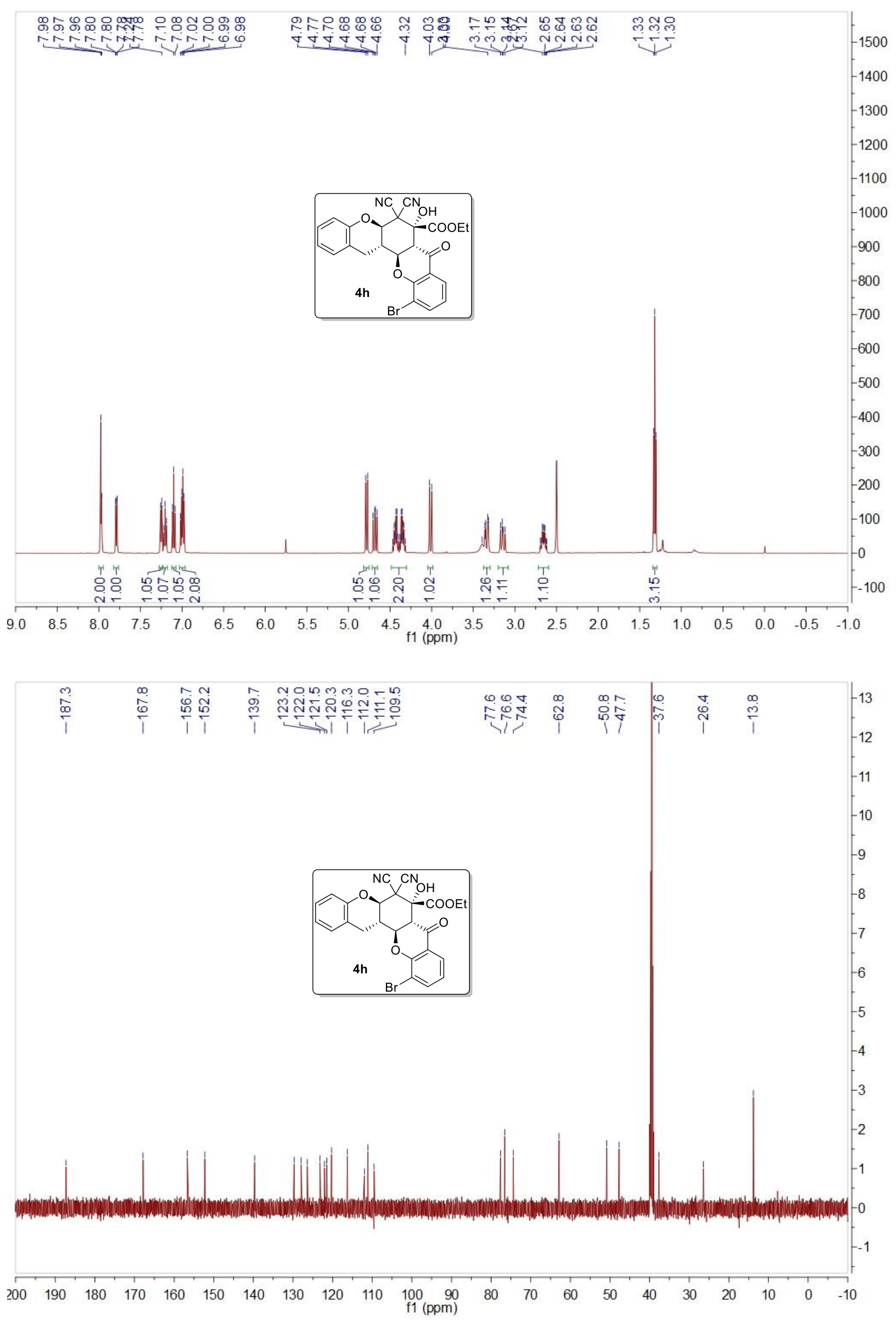

67 
Chrom Type: Fixed WL Chromatogram, $210 \mathrm{~nm}$

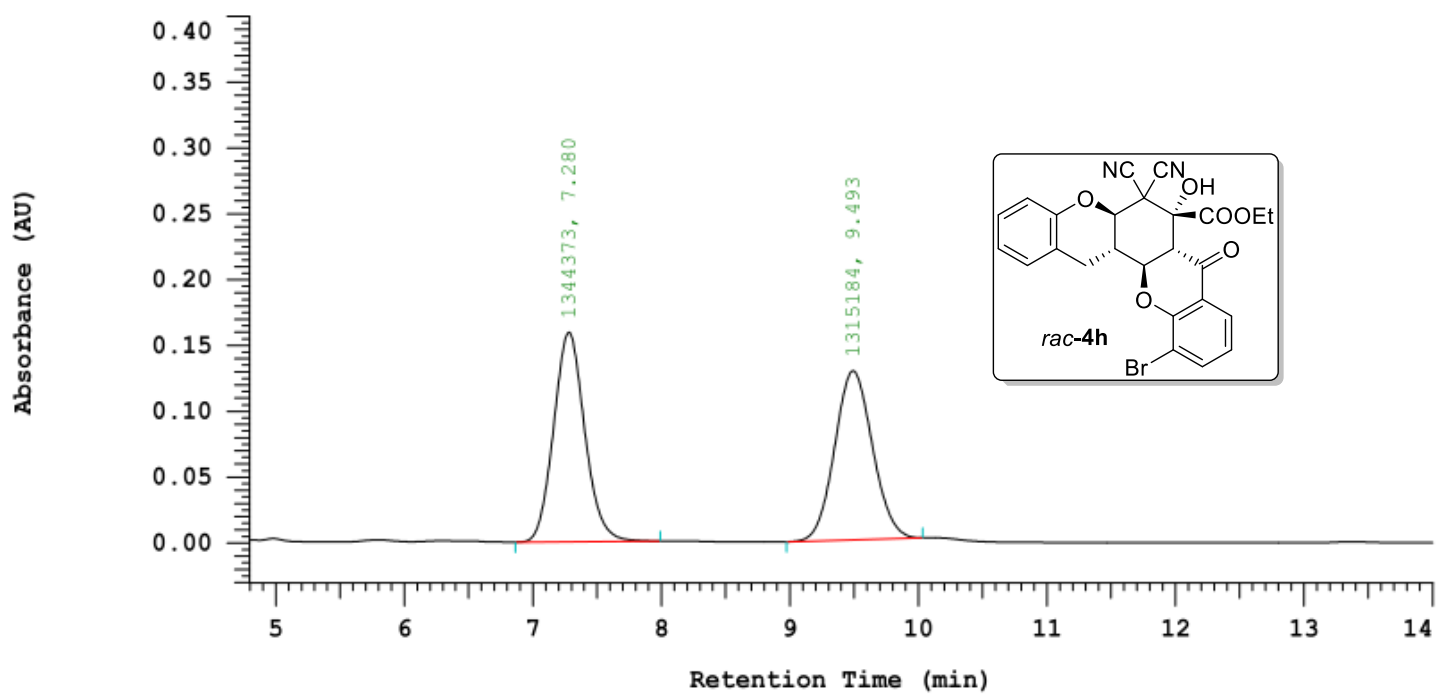

Chrom Type: Fixed WL Chromatogram, $210 \mathrm{~nm}$

Peak Quantitation: AREA

Calculation Method: AREA\%

\begin{tabular}{ccccc} 
No. & RT & Area & Area 응 & BC \\
\hline 1 & 7.280 & 1344373 & 50.549 & BB \\
2 & 9.493 & 1315184 & 49.451 & BB \\
\hline & & 2659557 & 100.000 & \\
\hline
\end{tabular}

Chrom Type: Fixed WL Chromatogram, 210 nm

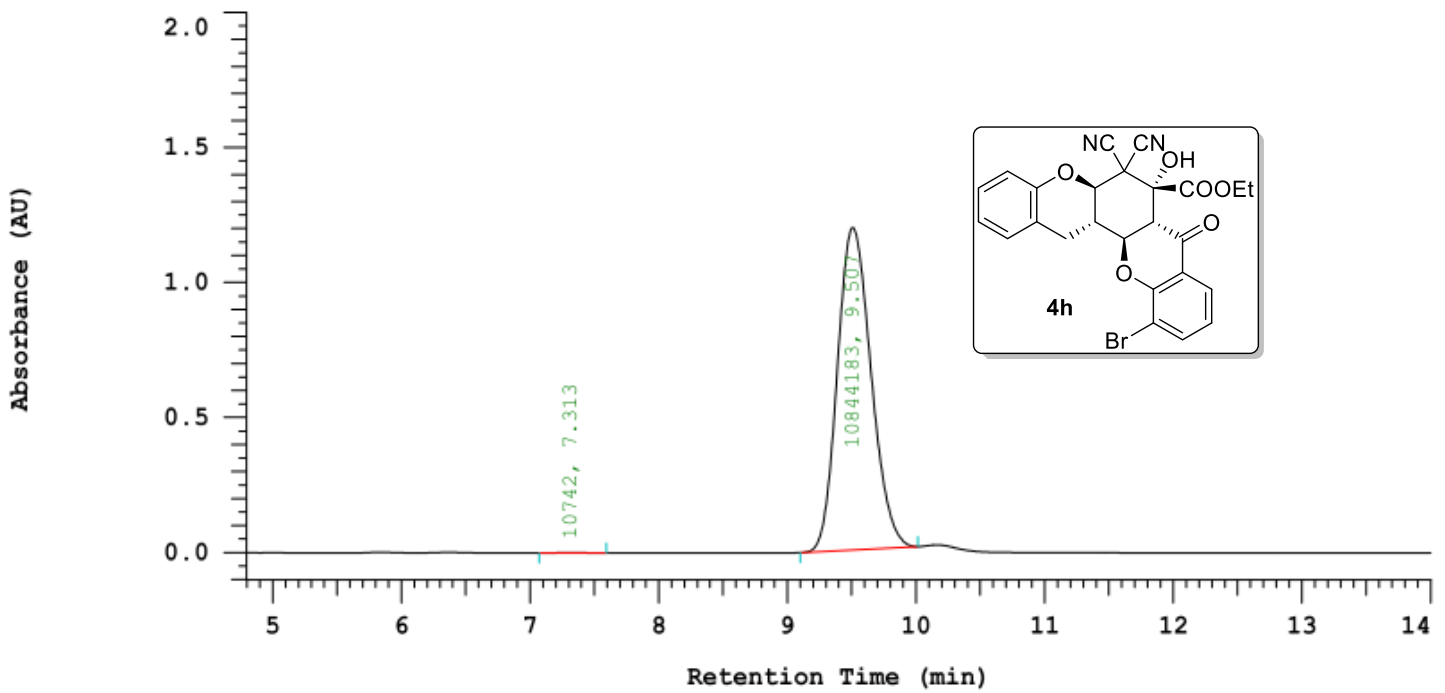

Peak Quantitation: AREA

Calculation Method: AREA응

\begin{tabular}{|c|c|c|c|c|}
\hline No. & $\mathrm{RT}$ & Area & Area $\frac{\circ}{\partial}$ & $\mathrm{BC}$ \\
\hline 1 & 7.313 & 10742 & 0.099 & BB \\
\hline 2 & 9.507 & 10844183 & 99.901 & $\mathrm{BB}$ \\
\hline & & 10854925 & 100.000 & \\
\hline
\end{tabular}



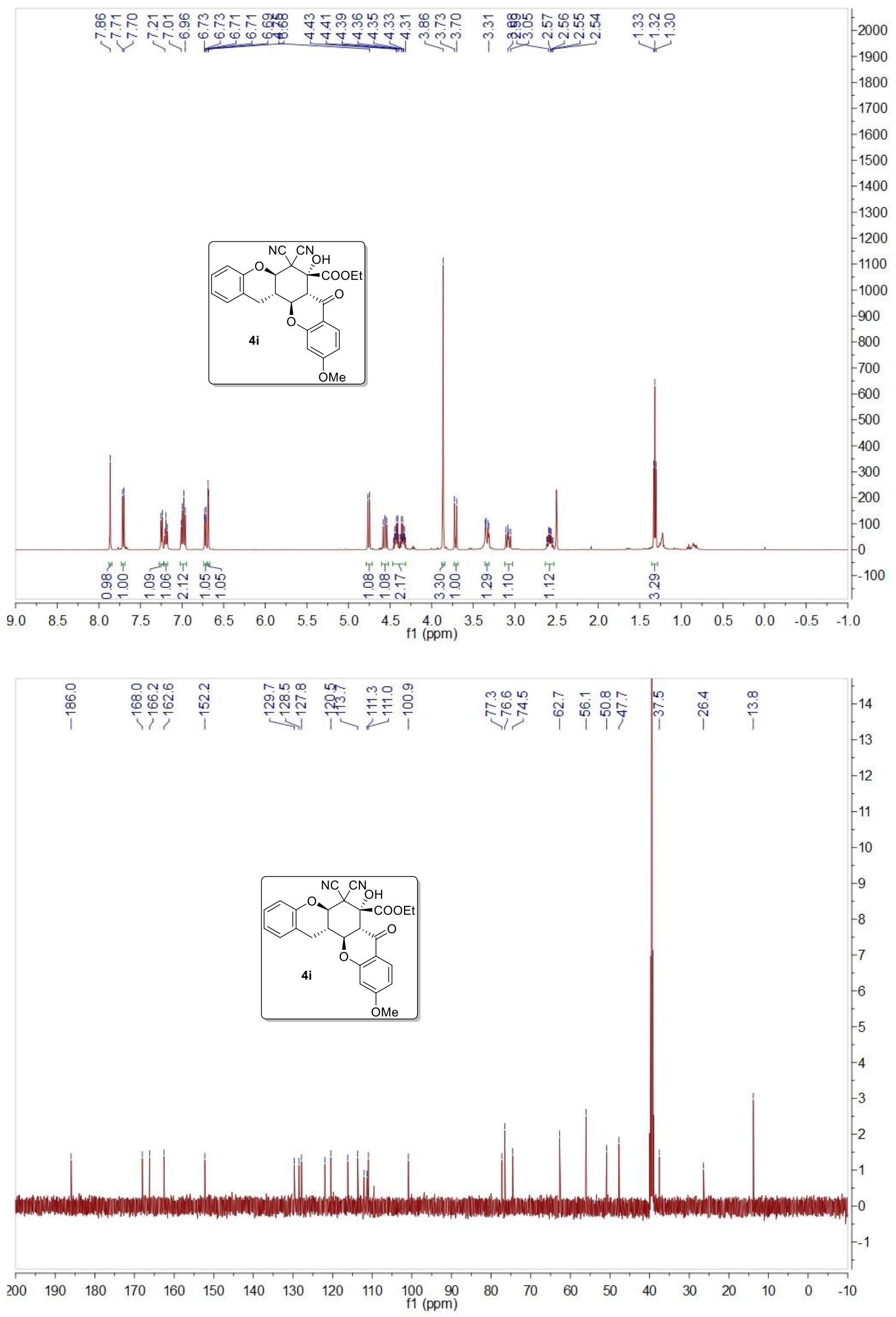
Chrom Type: Fixed WL Chromatogram, 210 nm

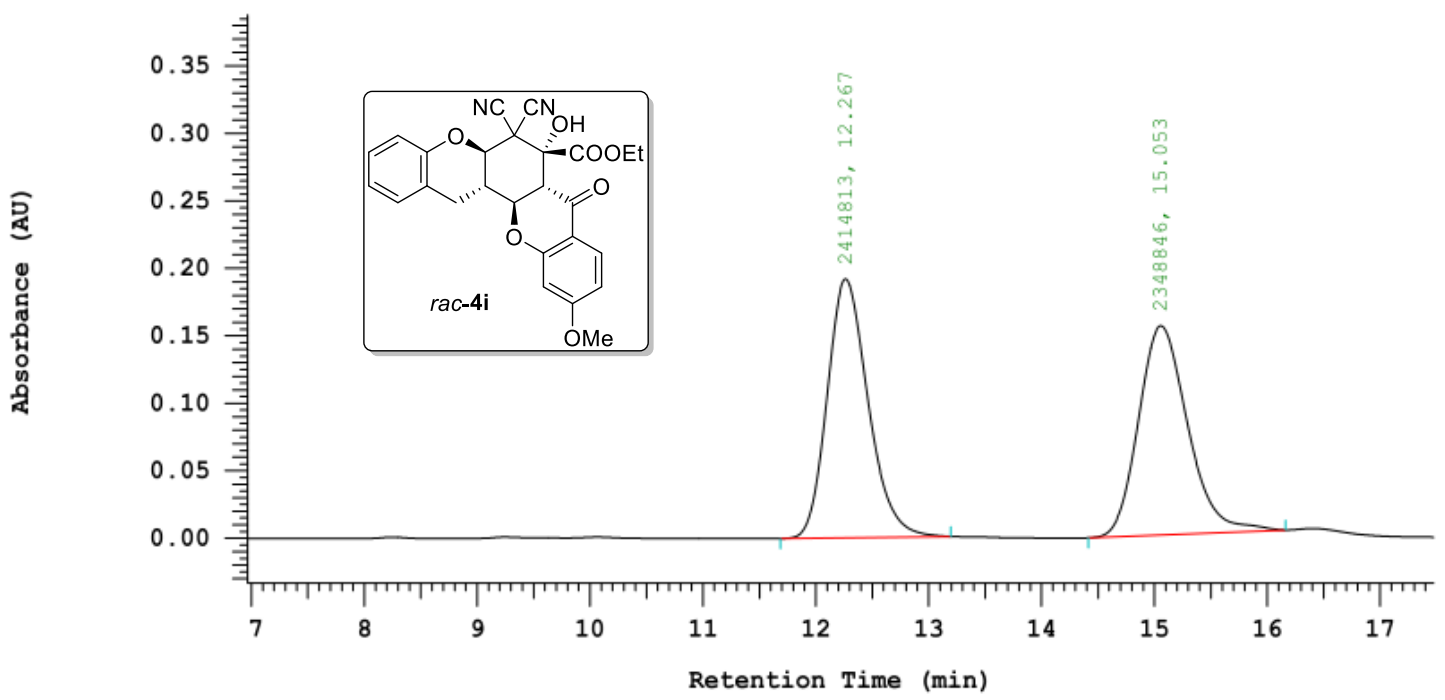

Chrom Type: Fixed WL Chromatogram, $210 \mathrm{~nm}$

Peak Quantitation: AREA

Calculation Method: AREA응

\begin{tabular}{ccccc} 
No. & RT & Area & Area & BC \\
\hline 1 & 12.267 & 2414813 & 50.692 & BB \\
2 & 15.053 & 2348846 & 49.308 & BB \\
\hline & & 4763659 & 100.000 & \\
\hline
\end{tabular}

Chrom Type: Fixed WL Chromatogram, 210 nm

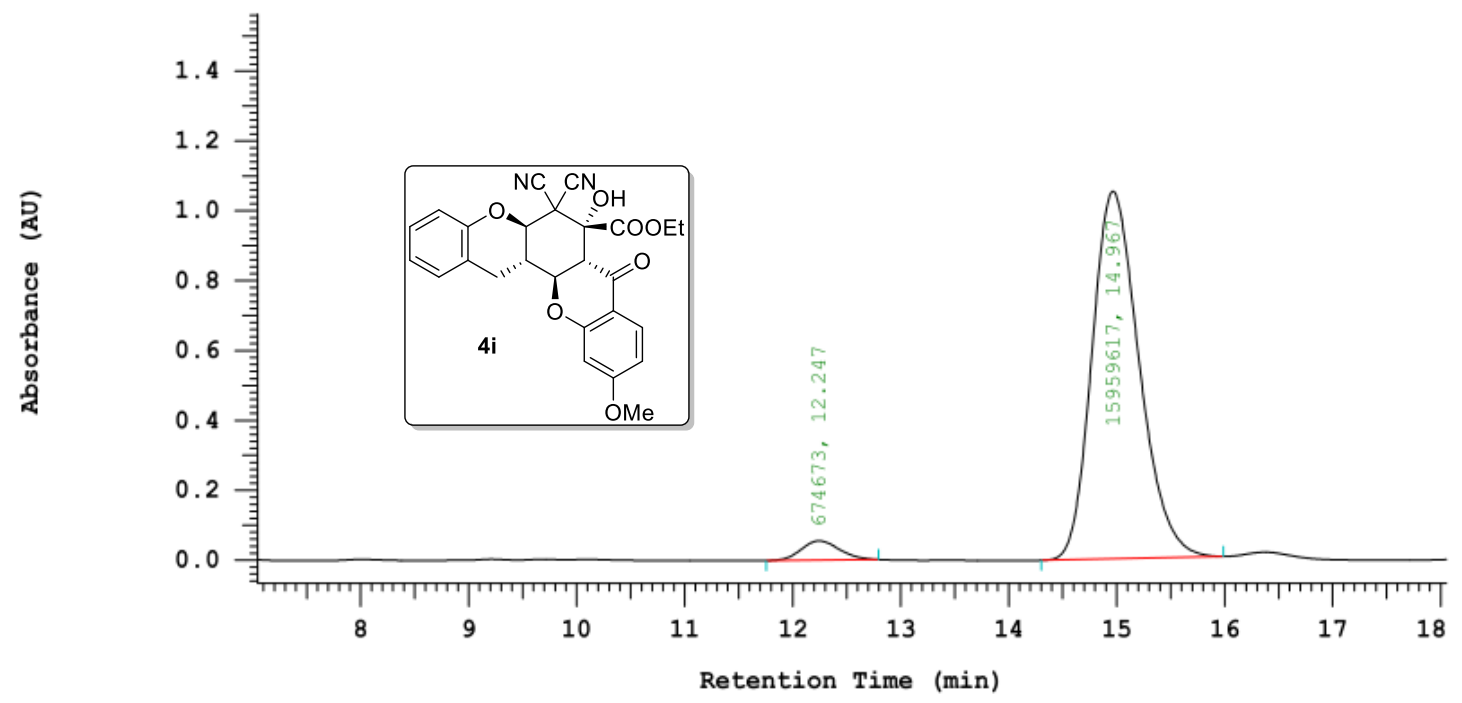

Chrom Type: Fixed WL Chromatogram, $210 \mathrm{~nm}$

Peak Quantitation: AREA

Calculation Method: AREA\%

\begin{tabular}{|c|c|c|c|c|}
\hline No. & RT & Area & Area $\frac{\circ}{0}$ & $\mathrm{BC}$ \\
\hline 1 & 12.247 & 674673 & 4.056 & $\mathrm{BB}$ \\
\hline 2 & 14.967 & 15959617 & 95.944 & $\mathrm{BB}$ \\
\hline & & 16634290 & 100.000 & \\
\hline
\end{tabular}



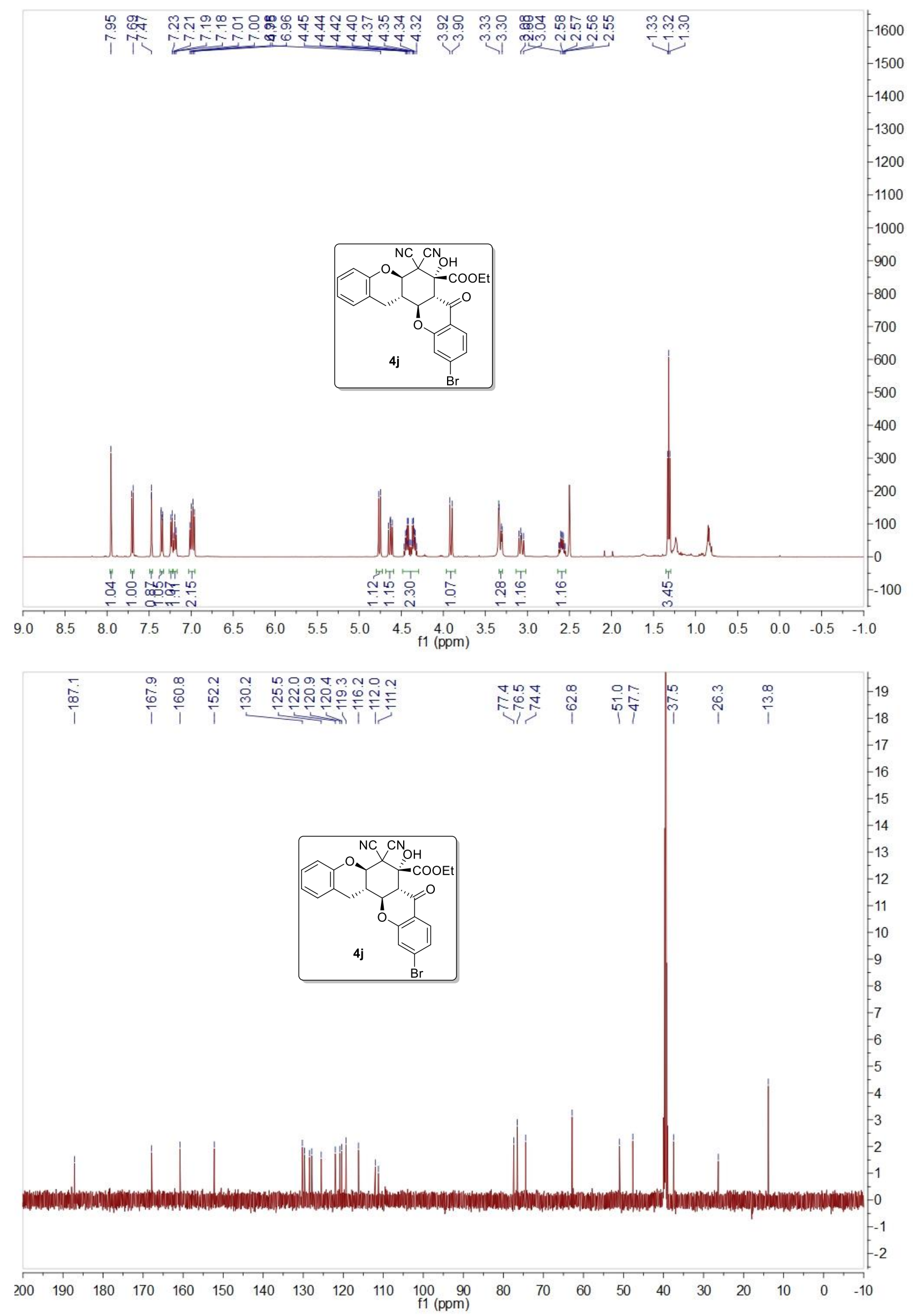
Chrom Type: Fixed WL Chromatogram, $210 \mathrm{~nm}$

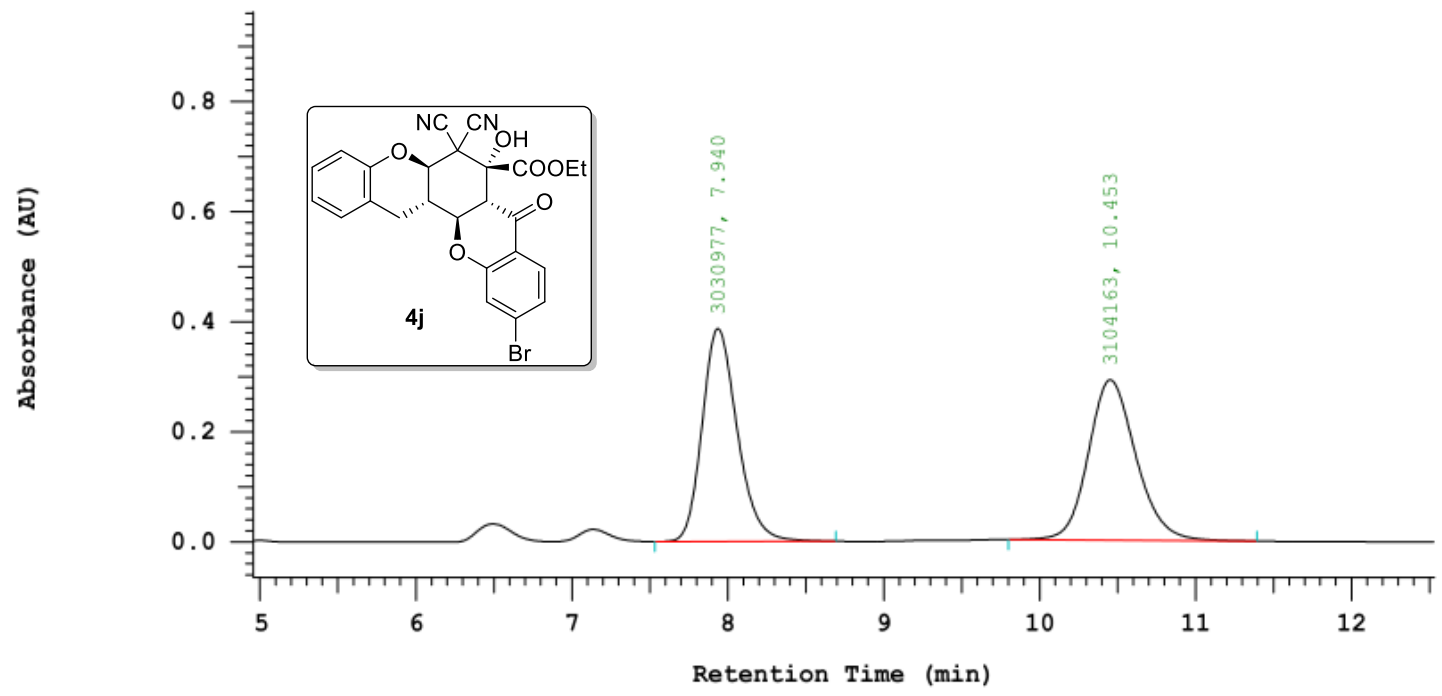

Chrom Type: Fixed WL Chromatogram, $210 \mathrm{~nm}$

Peak Quantitation: AREA

Calculation Method: AREA\%

\begin{tabular}{|c|c|c|c|c|}
\hline No. & $\mathrm{RT}$ & Area & Area \% & $\mathrm{BC}$ \\
\hline 1 & 7.940 & 3030977 & 49.404 & BB \\
\hline 2 & 10.453 & 3104163 & 50.596 & BB \\
\hline & & 6135140 & 100.000 & \\
\hline
\end{tabular}

Chrom Type: Fixed WL Chromatogram, 210 nm

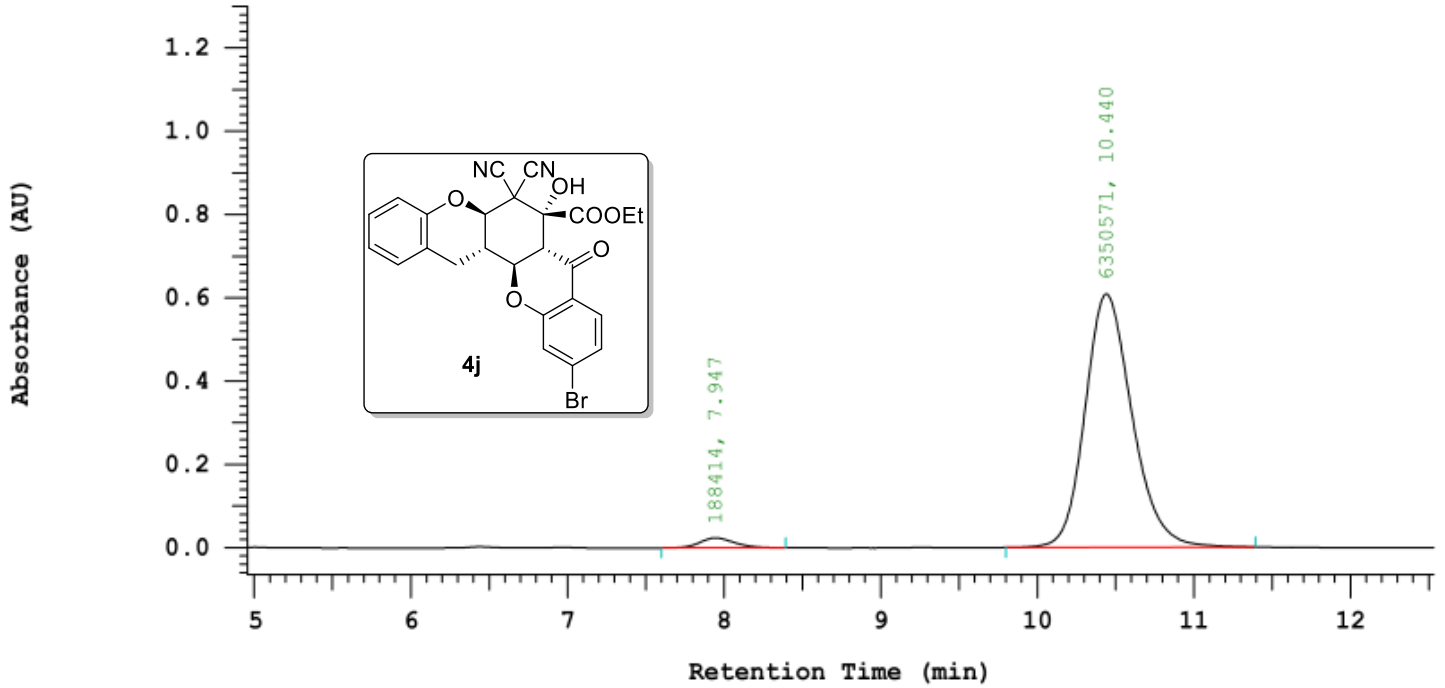

Chrom Type: Fixed WL Chromatogram, $210 \mathrm{~nm}$

Peak Quantitation: AREA

Calculation Method: AREA응

\begin{tabular}{|c|c|c|c|c|}
\hline No. & $\mathrm{RT}$ & Area & Area $\%$ & $\mathrm{BC}$ \\
\hline 1 & 7.947 & 188414 & 2.881 & $\mathrm{BB}$ \\
\hline \multirow[t]{2}{*}{2} & 10.440 & 6350571 & 97.119 & BB \\
\hline & & 6538985 & 100.000 & \\
\hline
\end{tabular}



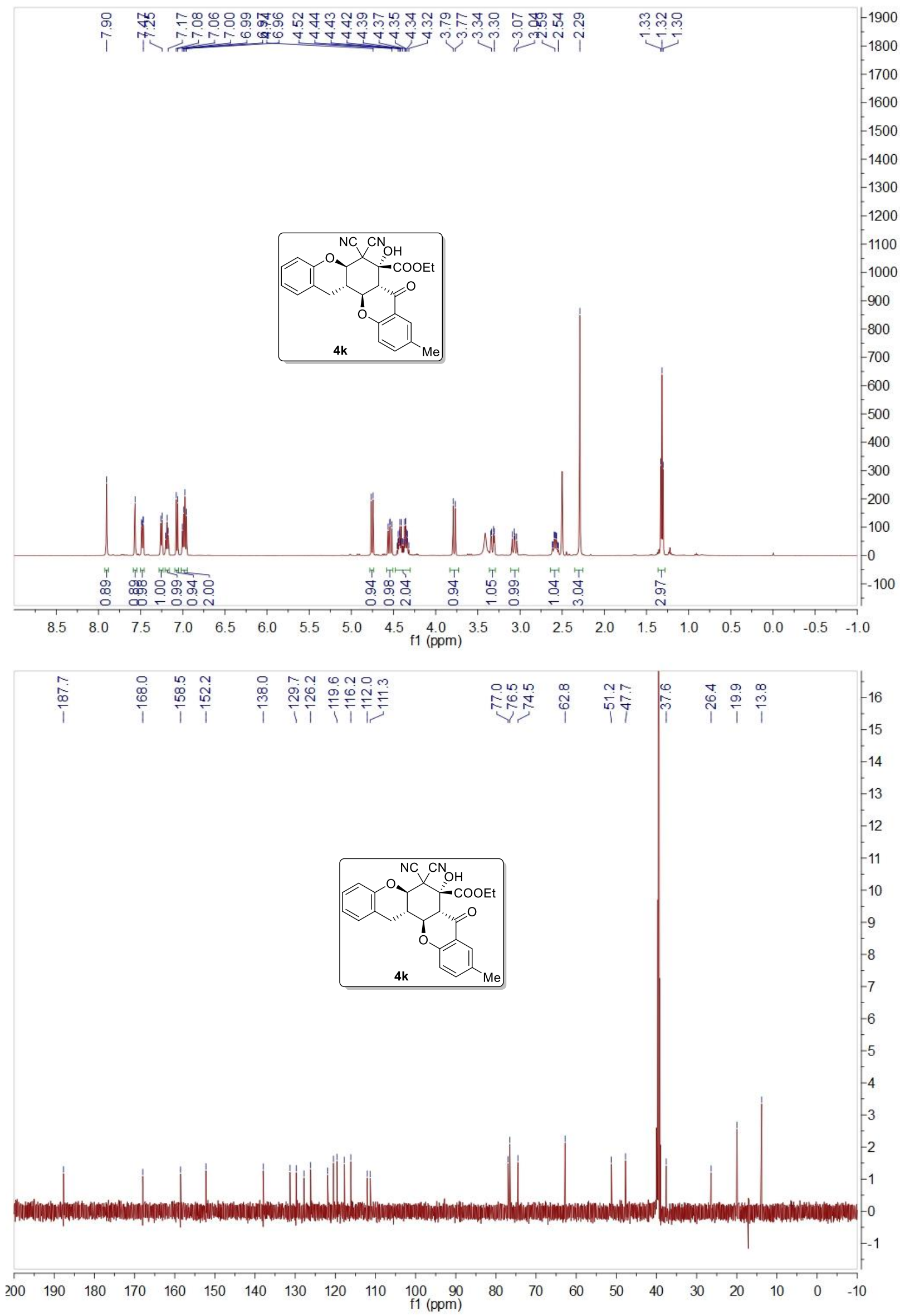
Chrom Type: Fixed WL Chromatogram, $210 \mathrm{~nm}$

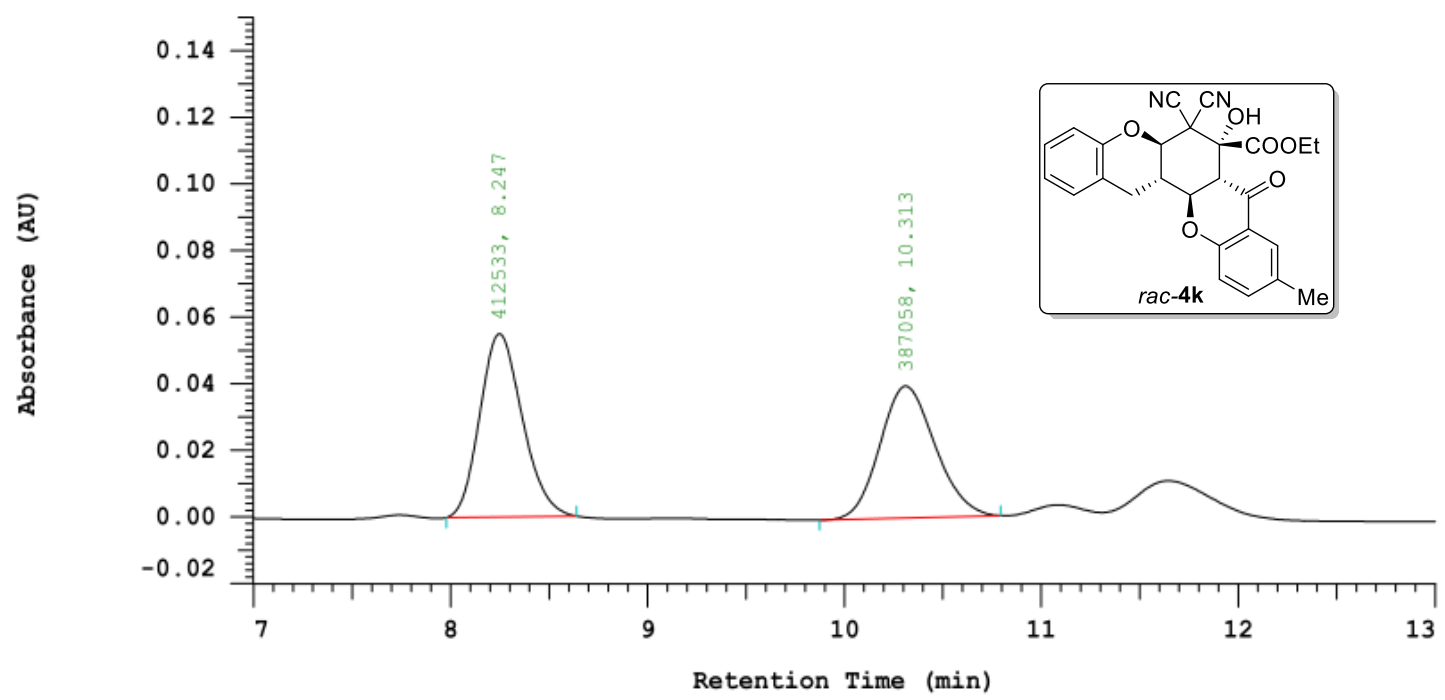

Chrom Type: Fixed WL Chromatogram, $210 \mathrm{~nm}$

Peak Quantitation: AREA

Calculation Method: AREA

\begin{tabular}{|c|c|c|c|c|}
\hline No. & RT & Area & Area & $\mathrm{BC}$ \\
\hline 1 & 8.247 & 412533 & 51.593 & $\mathrm{BB}$ \\
\hline 2 & 10.313 & 387058 & 48.407 & BB \\
\hline & & 799591 & 100.000 & \\
\hline
\end{tabular}

Chrom Type: Fixed WL Chromatogram, 210 nm

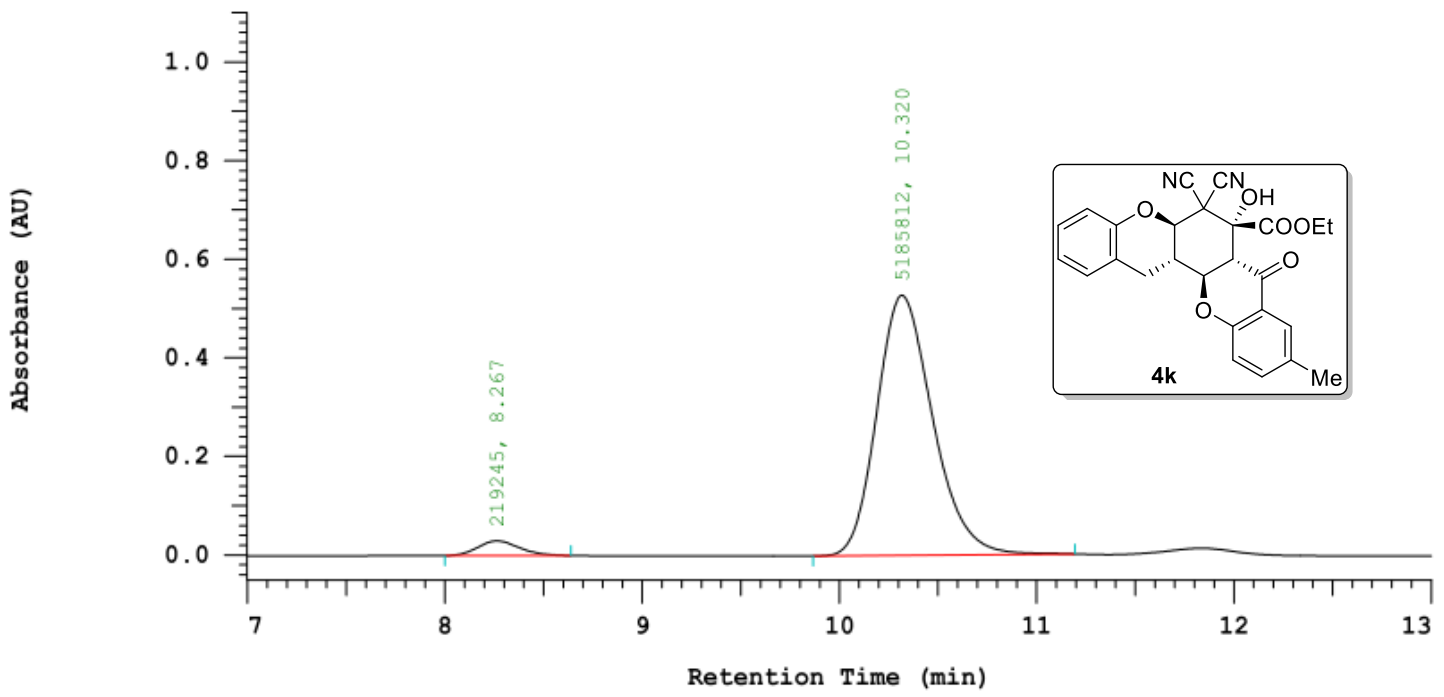

Chrom Type: Fixed WL Chromatogram, $210 \mathrm{~nm}$

Peak Quantitation: AREA

Calculation Method: AREA응

\begin{tabular}{|c|c|c|c|c|}
\hline No. & $\mathrm{RT}$ & Area & Area $\%$ & $\mathrm{BC}$ \\
\hline 1 & 8.267 & 219245 & 4.056 & BB \\
\hline 2 & 10.320 & 5185812 & 95.944 & BB \\
\hline & & 5405057 & 100.000 & \\
\hline
\end{tabular}



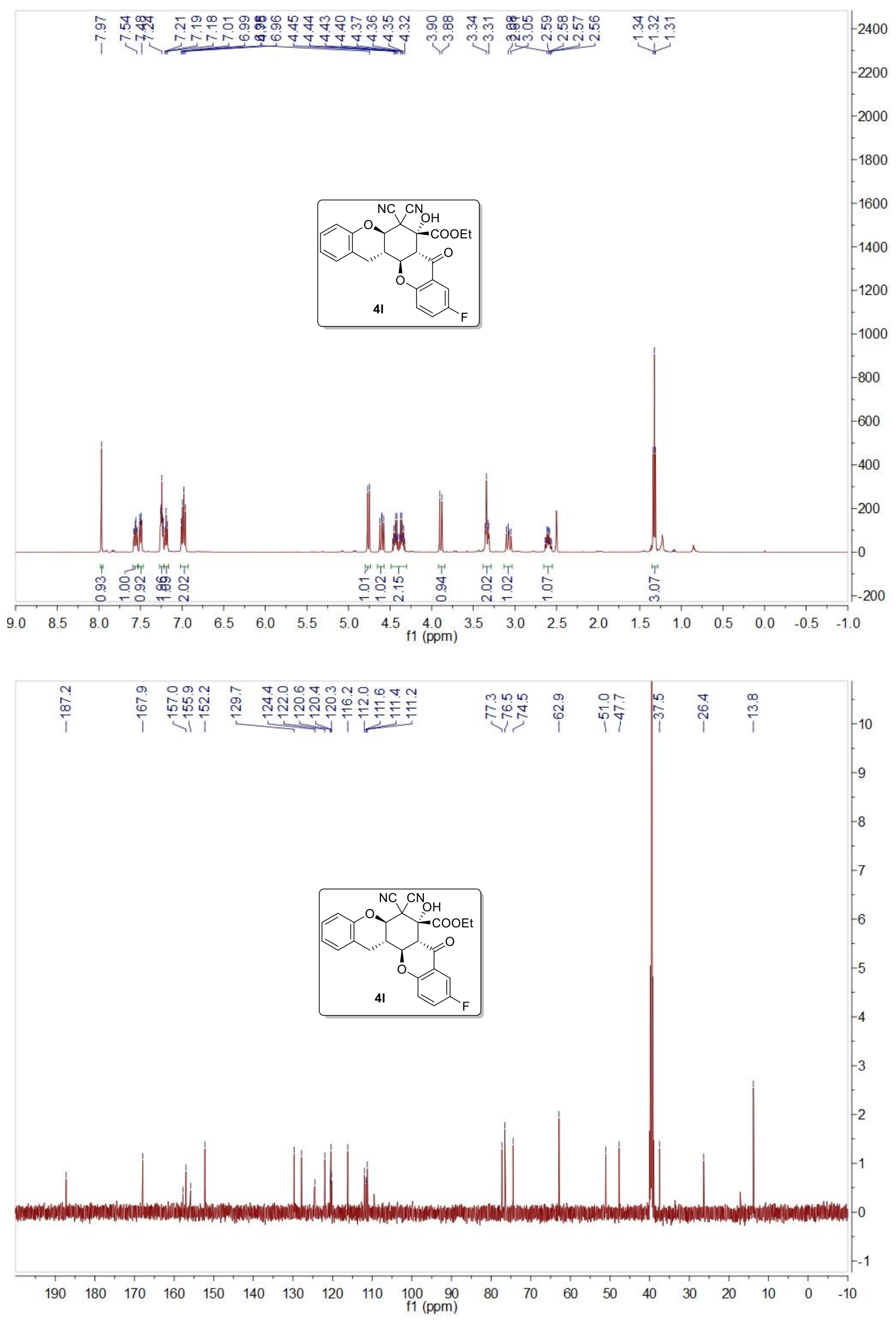
Chrom Type: Fixed WL Chromatogram, 210 nm

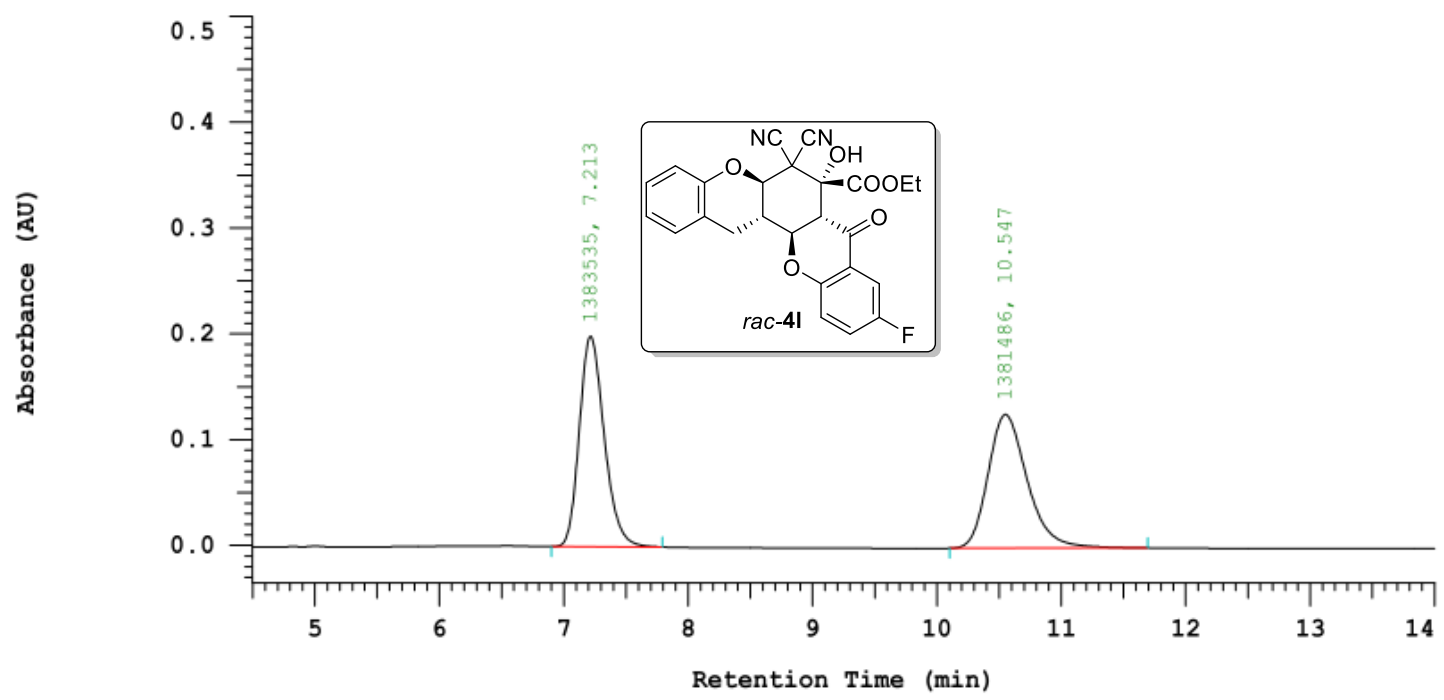

Chrom Type: Fixed WL Chromatogram, $210 \mathrm{~nm}$

Peak Quantitation: AREA

Calculation Method: AREA\%

\begin{tabular}{|c|c|c|c|c|}
\hline No. & $\mathrm{RT}$ & Area & Area & $\mathrm{BC}$ \\
\hline 1 & 7.213 & 1383535 & 50.037 & BB \\
\hline 2 & 10.547 & 1381486 & 49.963 & $\mathrm{BB}$ \\
\hline & & 2765021 & 100.000 & \\
\hline
\end{tabular}

Chrom Type: Fixed WL Chromatogram, $210 \mathrm{~nm}$

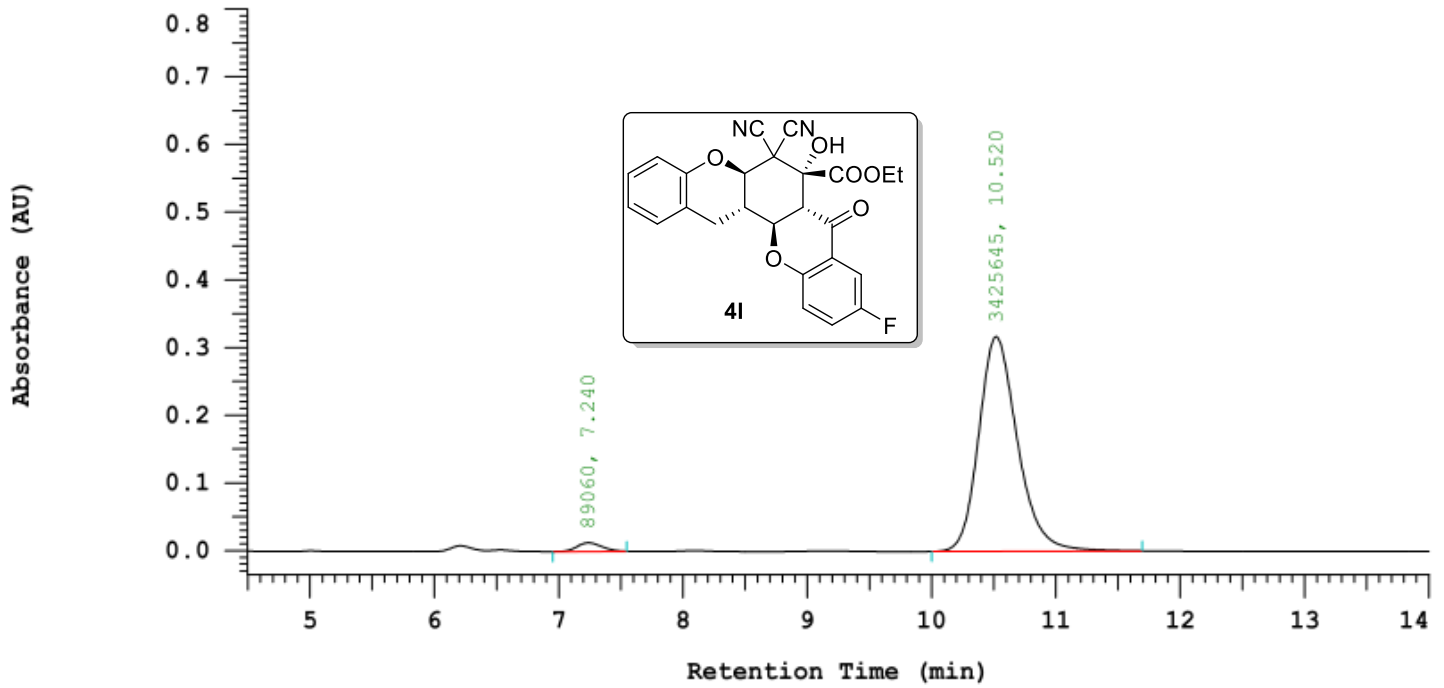

Chrom Type: Fixed WL Chromatogram, $210 \mathrm{~nm}$

Peak Quantitation: AREA

Calculation Method: AREA응

\begin{tabular}{|c|c|c|c|c|}
\hline No. & RT & Area & Area \% & $\mathrm{BC}$ \\
\hline 1 & 7.240 & 89060 & 2.534 & BB \\
\hline 2 & 10.520 & 3425645 & 97.466 & BB \\
\hline & & 3514705 & 100.000 & \\
\hline
\end{tabular}



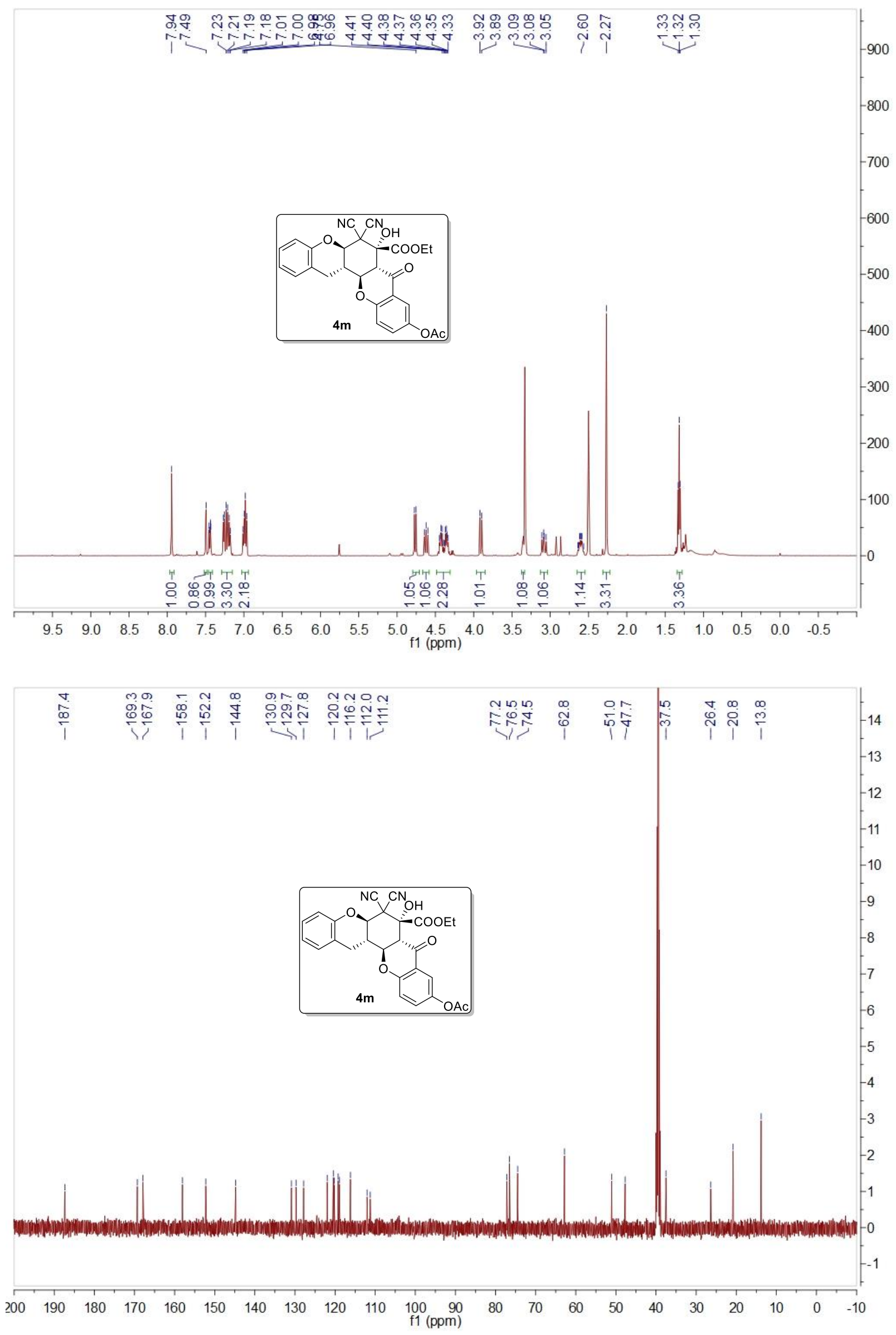
Chrom Type: Fixed WL Chromatogram, $210 \mathrm{~nm}$

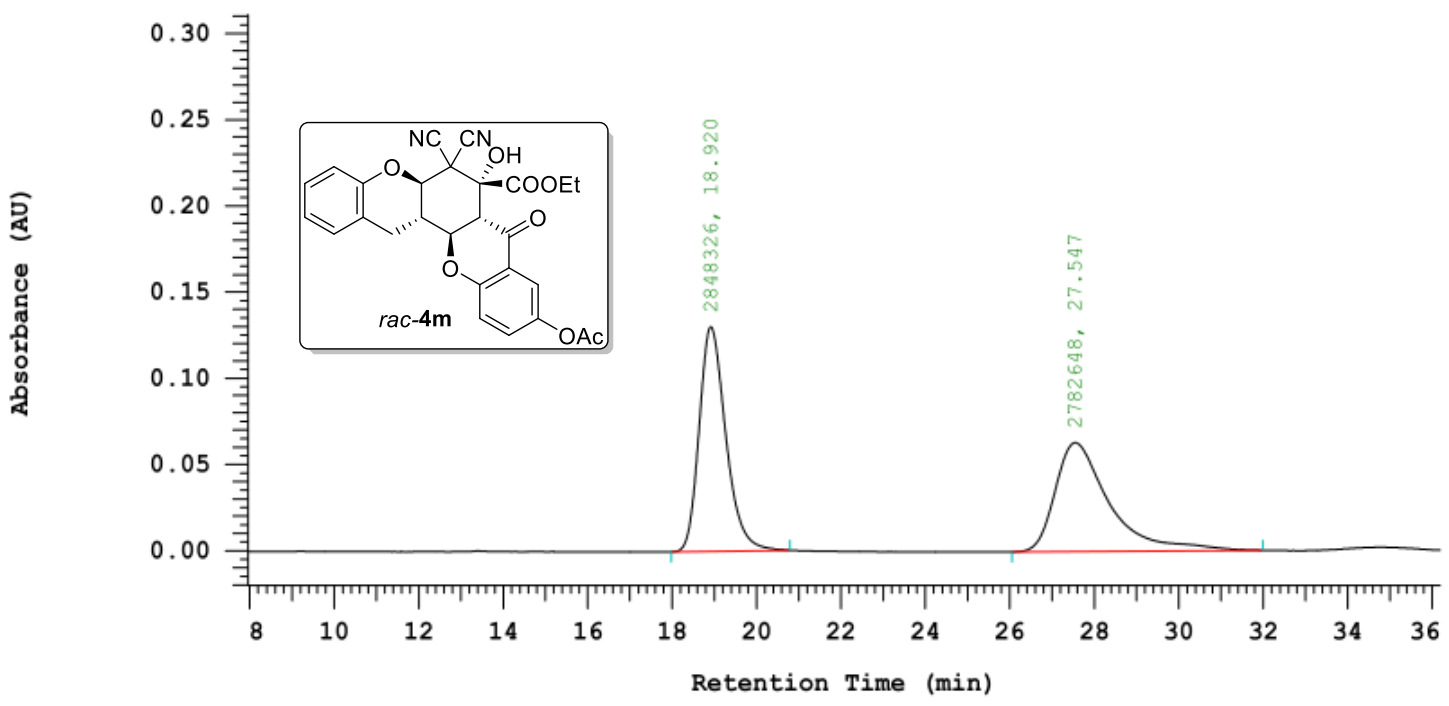

Chrom Type: Fixed WL Chromatogram, $210 \mathrm{~nm}$

Peak Quantitation: AREA

Calculation Method: AREA음

\begin{tabular}{ccccc} 
No. & RT & Area & Area & BC \\
\hline 1 & 18.920 & 2848326 & 50.583 & BB \\
2 & 27.547 & 2782648 & 49.417 & BB \\
\hline & & 5630974 & 100.000 & \\
\hline
\end{tabular}

Chrom Type: Fixed WL Chromatogram, 210 nm

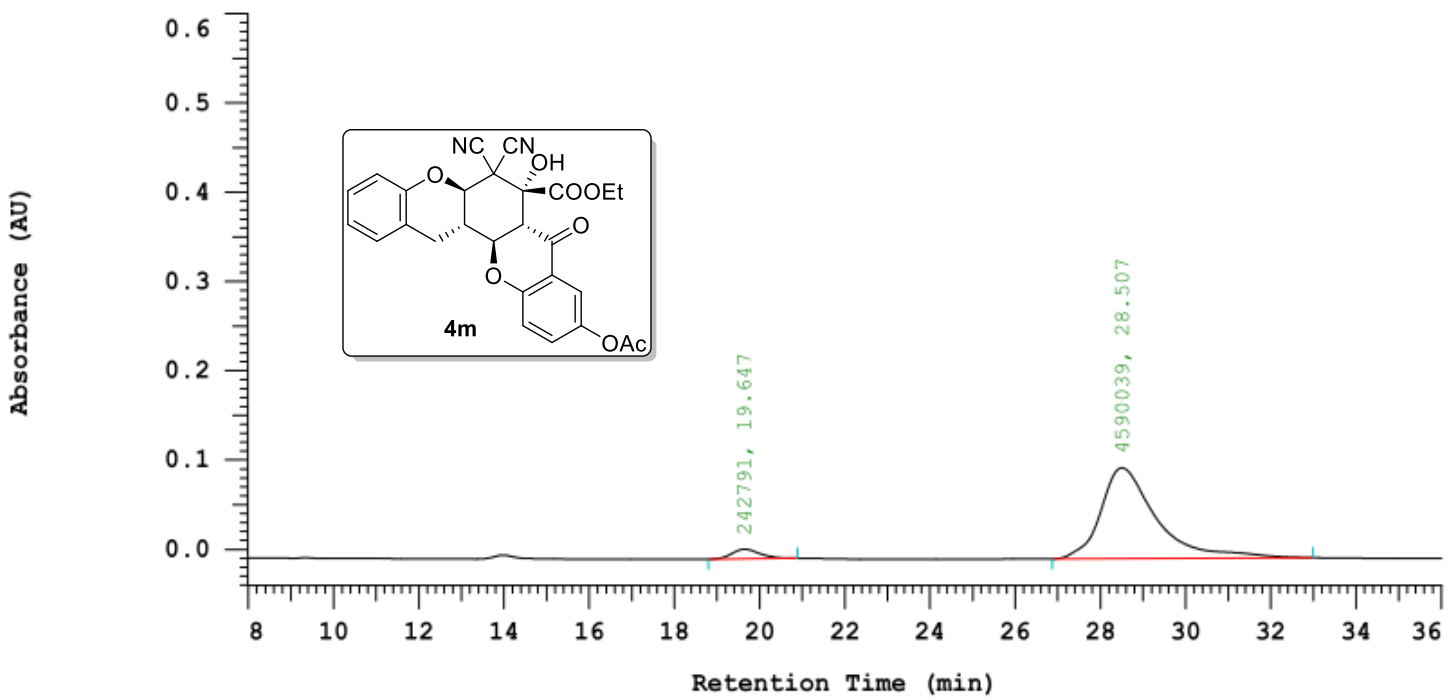

Chrom Type: Fixed WL Chromatogram, $210 \mathrm{~nm}$

Peak Quantitation: AREA

Calculation Method: AREA응

\begin{tabular}{|c|c|c|c|c|}
\hline No. & $\mathrm{RT}$ & Area & Area $\frac{\circ}{0}$ & $\mathrm{BC}$ \\
\hline 1 & 19.647 & 242791 & 5.024 & $\mathrm{BB}$ \\
\hline 2 & 28.507 & 4590039 & 94.976 & $\mathrm{BB}$ \\
\hline & & 4832830 & 100.000 & \\
\hline
\end{tabular}



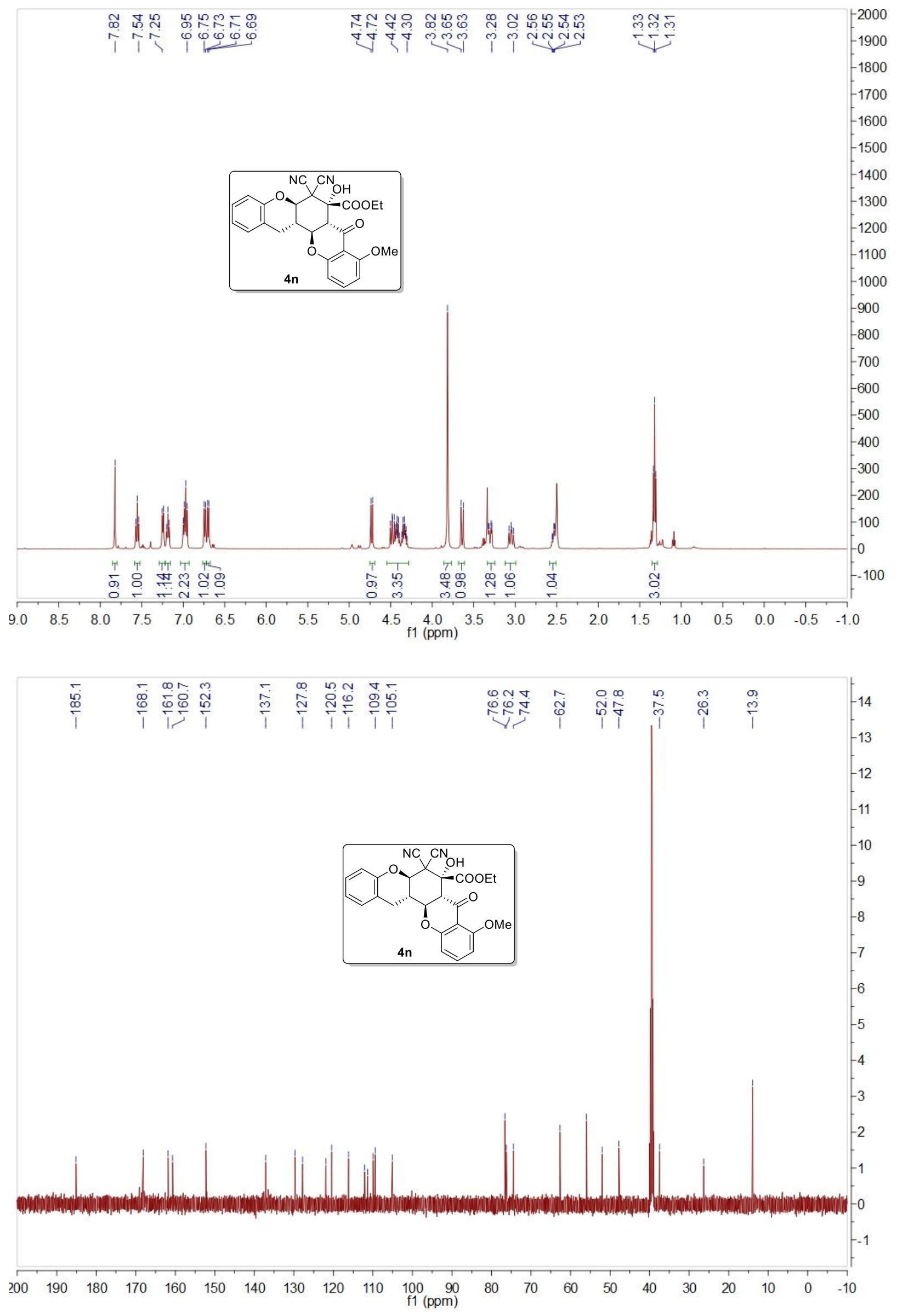
Chrom Type: Fixed WL Chromatogram, 210 nm

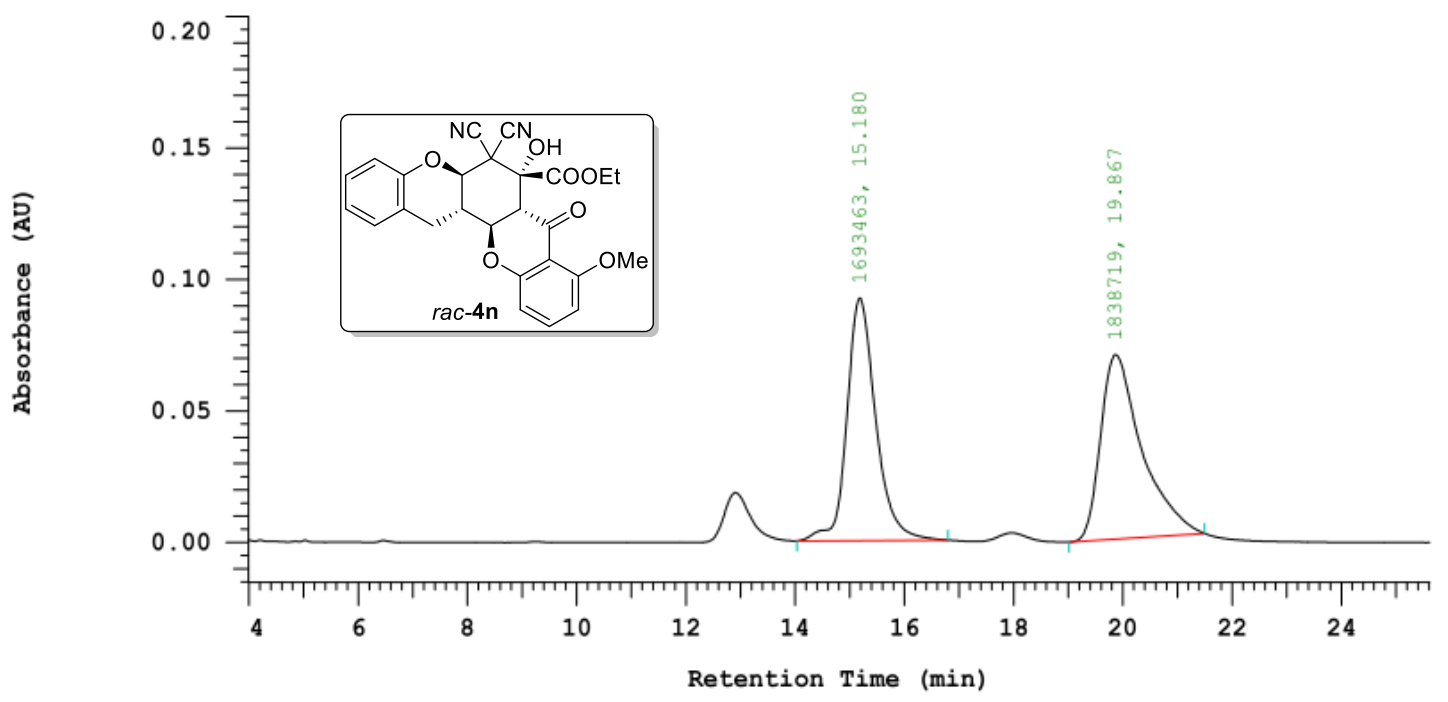

Chrom Type: Fixed WL Chromatogram, $210 \mathrm{~nm}$

Peak Quantitation: AREA

Calculation Method: AREA\%

\begin{tabular}{ccccc} 
No. & RT & Area & Area & BC \\
\hline 1 & 15.180 & 1693463 & 47.944 & BB \\
2 & 19.867 & 1838719 & 52.056 & BB \\
\hline & 3532182 & 100.000 & \\
\hline
\end{tabular}

Chrom Type: Fixed WL Chromatogram, $210 \mathrm{~nm}$

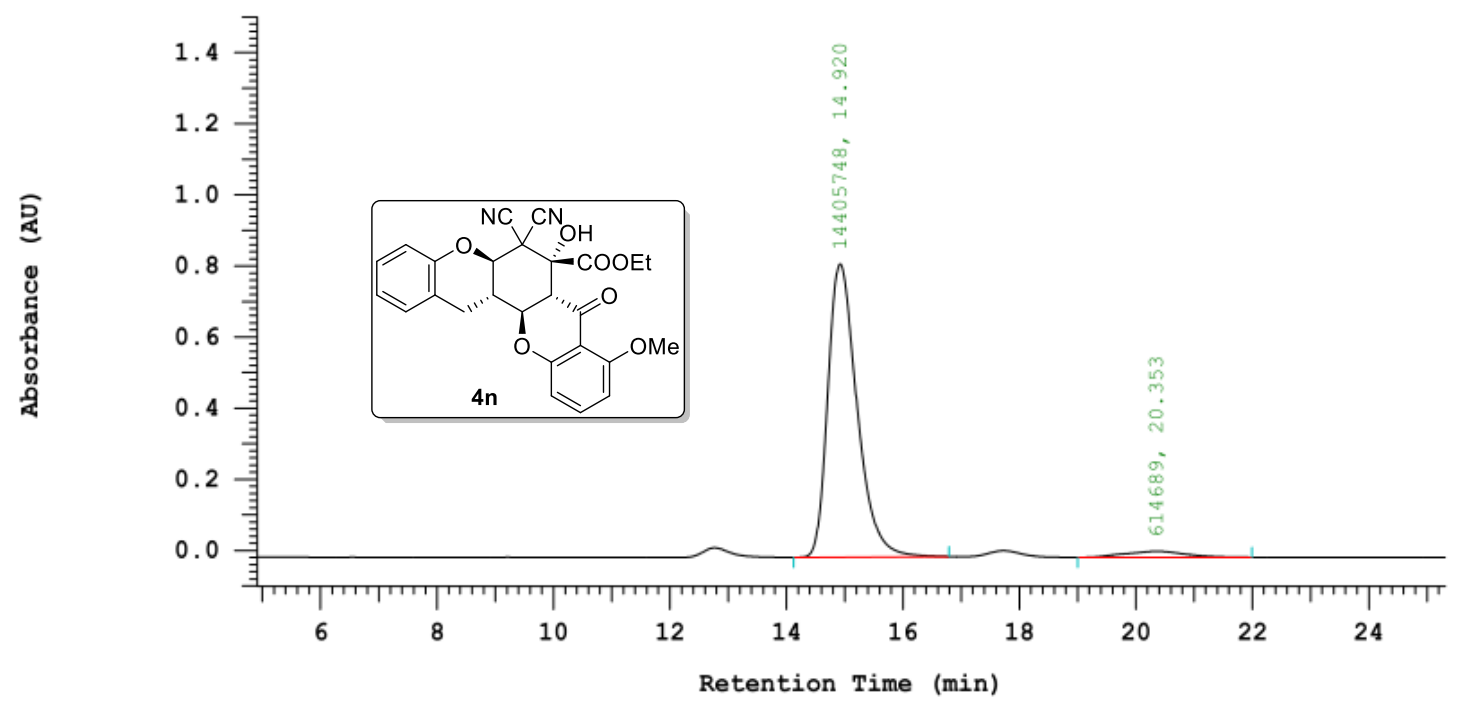

Chrom Type: Fixed WL Chromatogram, $210 \mathrm{~nm}$

Peak Quantitation: AREA

Calculation Method: AREA\%

\begin{tabular}{|c|c|c|c|c|}
\hline No. & RT & Area & Area $\%$ & $\mathrm{BC}$ \\
\hline 1 & 14.920 & 14405748 & 95.908 & $\mathrm{BB}$ \\
\hline 2 & 20.353 & 614689 & 4.092 & $\mathrm{BB}$ \\
\hline & & 15020437 & 100.000 & \\
\hline
\end{tabular}



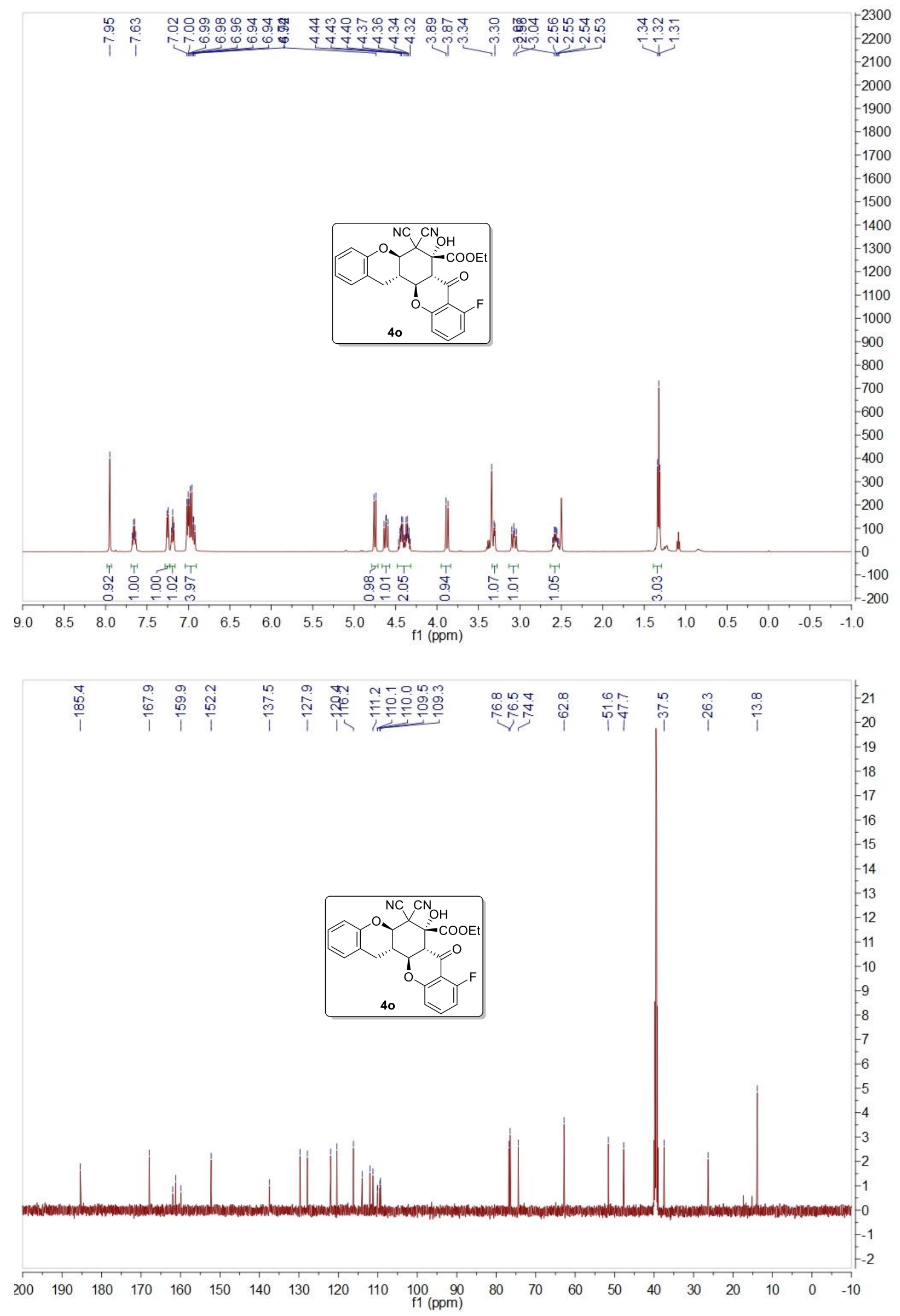

81 
Chrom Type: Fixed WL Chromatogram, $210 \mathrm{~nm}$

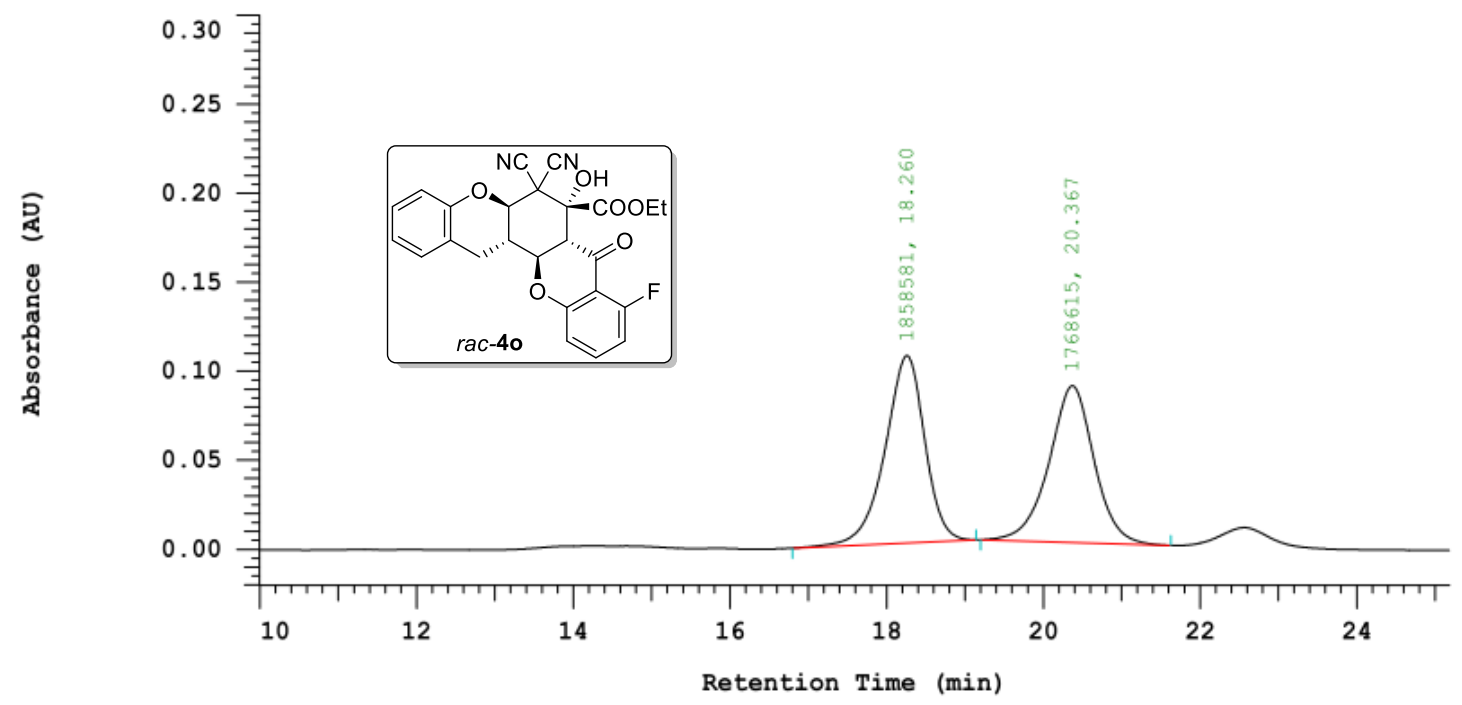

Chrom Type: Fixed WL Chromatogram, $210 \mathrm{~nm}$

Peak Quantitation: AREA

Calculation Method: AREA응

\begin{tabular}{ccccc} 
No. & RT & Area & Area & BC \\
\hline 1 & 18.260 & 1858581 & 51.240 & BB \\
2 & 20.367 & 1768615 & 48.760 & BB \\
\hline & & 3627196 & 100.000 & \\
\hline
\end{tabular}

Chrom Type: Fixed WL Chromatogram, 210 nm

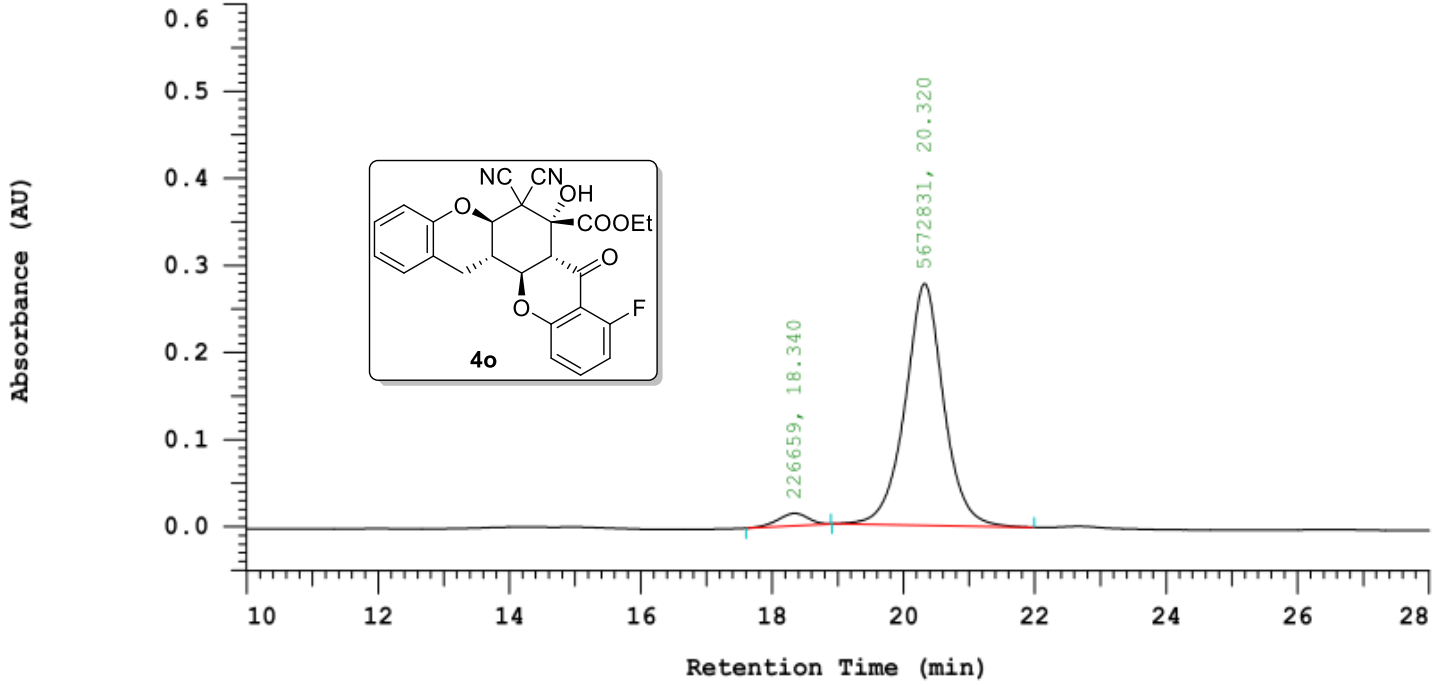

Chrom Type: Fixed WL Chromatogram, $210 \mathrm{~nm}$

Peak Quantitation: AREA

Calculation Method: AREA음

\begin{tabular}{|c|c|c|c|c|}
\hline No. & $\mathrm{RT}$ & Area & Area $\frac{\circ}{0}$ & $\mathrm{BC}$ \\
\hline 1 & 18.340 & 226659 & 3.842 & $\mathrm{BB}$ \\
\hline 2 & 20.320 & 5672831 & 96.158 & $\mathrm{BB}$ \\
\hline & & 5899490 & 100.000 & \\
\hline
\end{tabular}



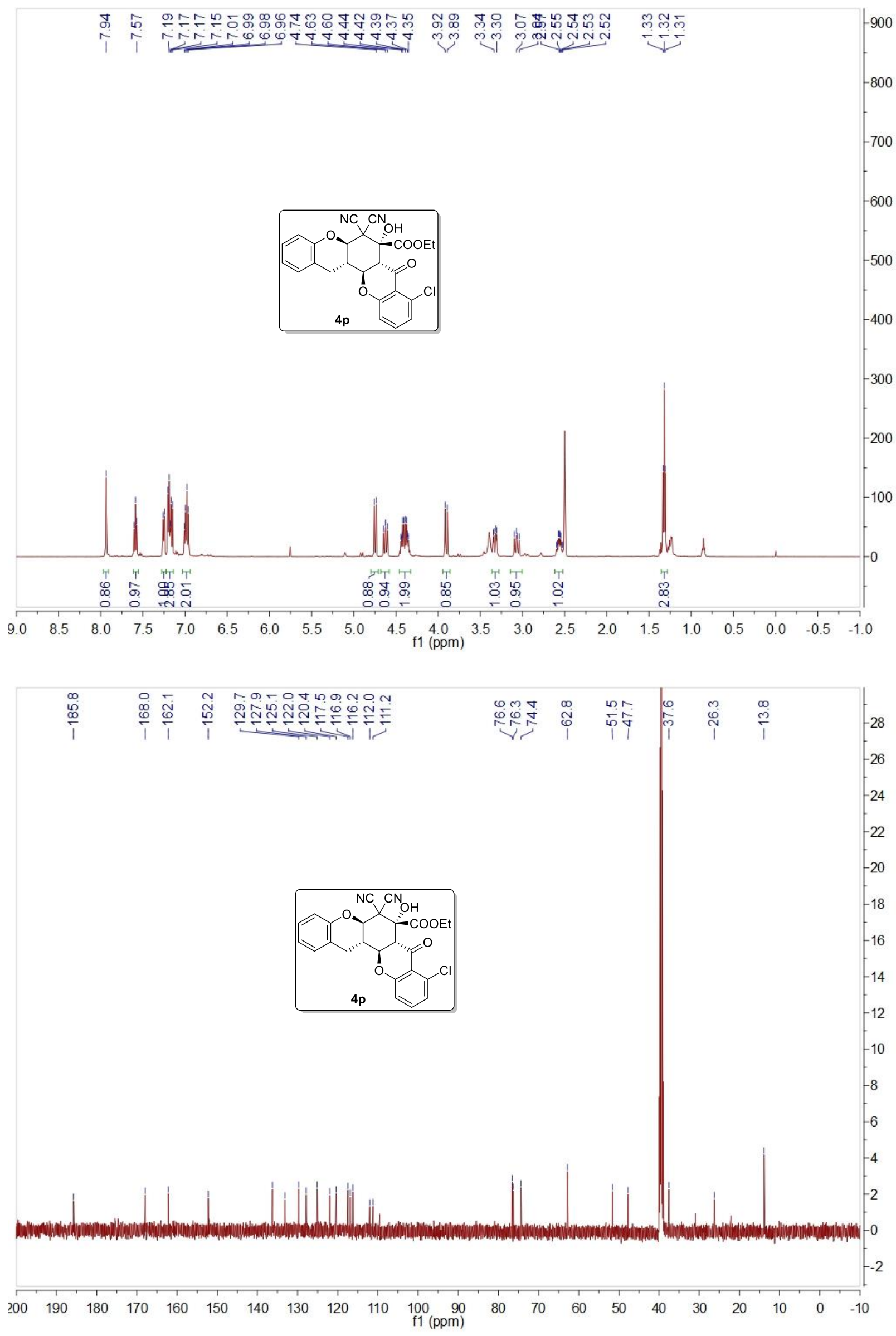
Chrom Type: Fixed WL Chromatogram, 210 nm

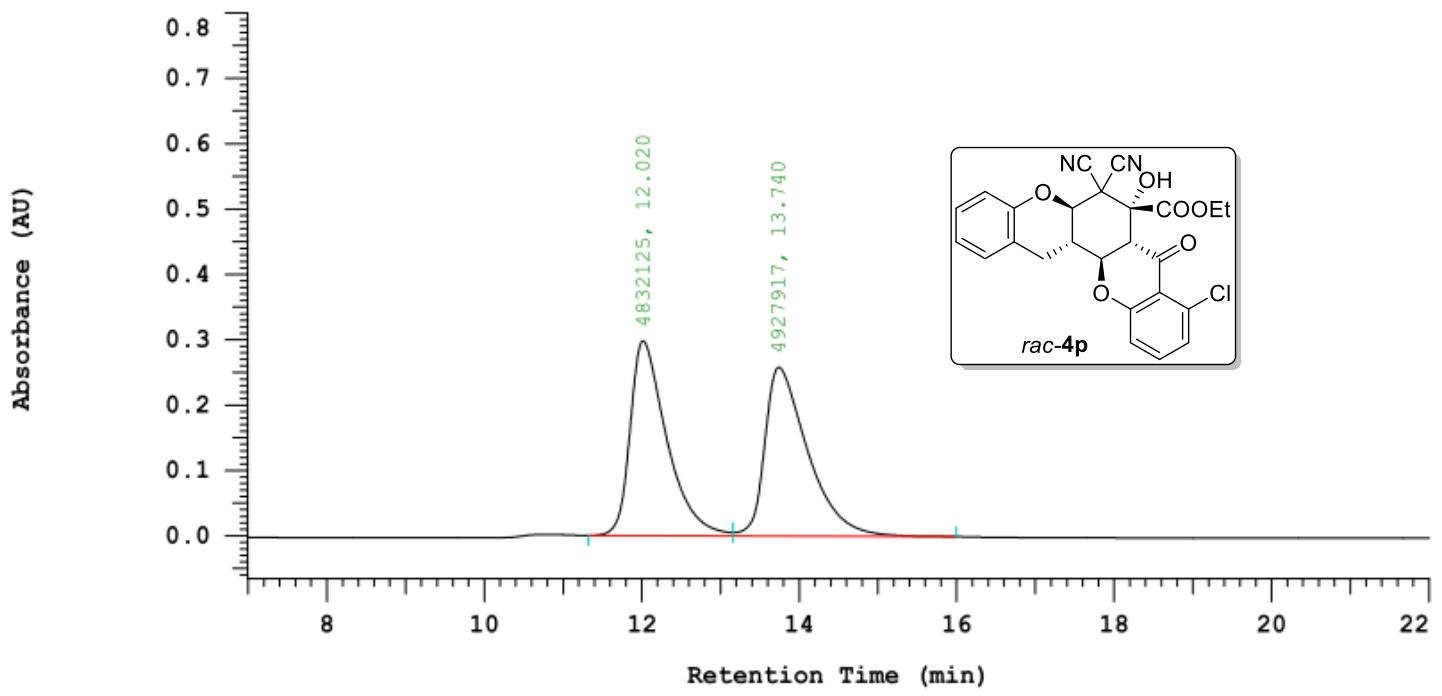

Chrom Type: Fixed WL Chromatogram, $210 \mathrm{~nm}$

Peak Quantitation: AREA

Calculation Method: AREA응

\begin{tabular}{|c|c|c|c|c|}
\hline No. & RT & Area & Area & $\mathrm{BC}$ \\
\hline 1 & 12.020 & 4832125 & 49.509 & BV \\
\hline 2 & 13.740 & 4927917 & 50.491 & VB \\
\hline & & 9760042 & 100.000 & \\
\hline
\end{tabular}

Chrom Type: Fixed WL Chromatogram, $210 \mathrm{~nm}$

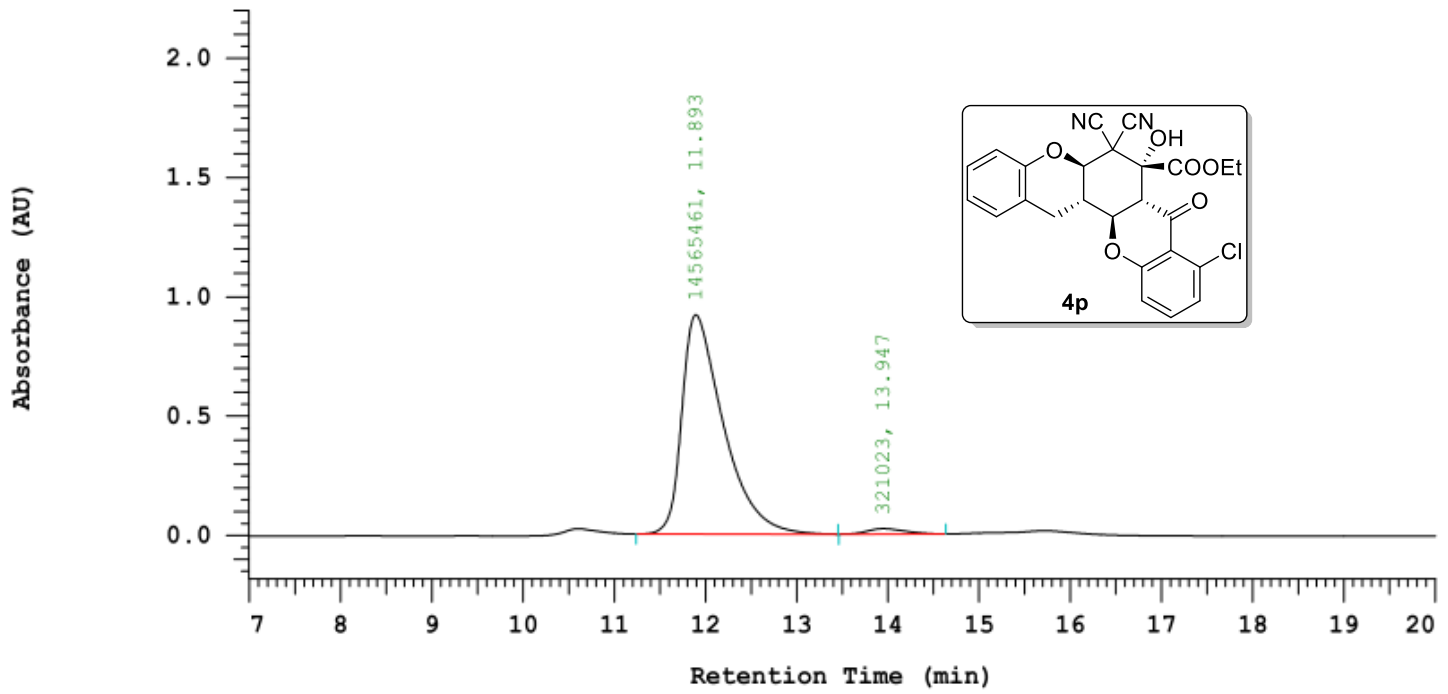

Chrom Type: Fixed WL Chromatogram, $210 \mathrm{~nm}$

Peak Quantitation: AREA

Calculation Method: AREA응

\begin{tabular}{|c|c|c|c|c|}
\hline No. & $\mathrm{RT}$ & Area & Area $\%$ & $\mathrm{BC}$ \\
\hline 1 & 11.893 & 14565461 & 97.844 & $\mathrm{BB}$ \\
\hline 2 & 13.947 & 321023 & 2.156 & $\mathrm{BB}$ \\
\hline & & 14886484 & 100.000 & \\
\hline
\end{tabular}



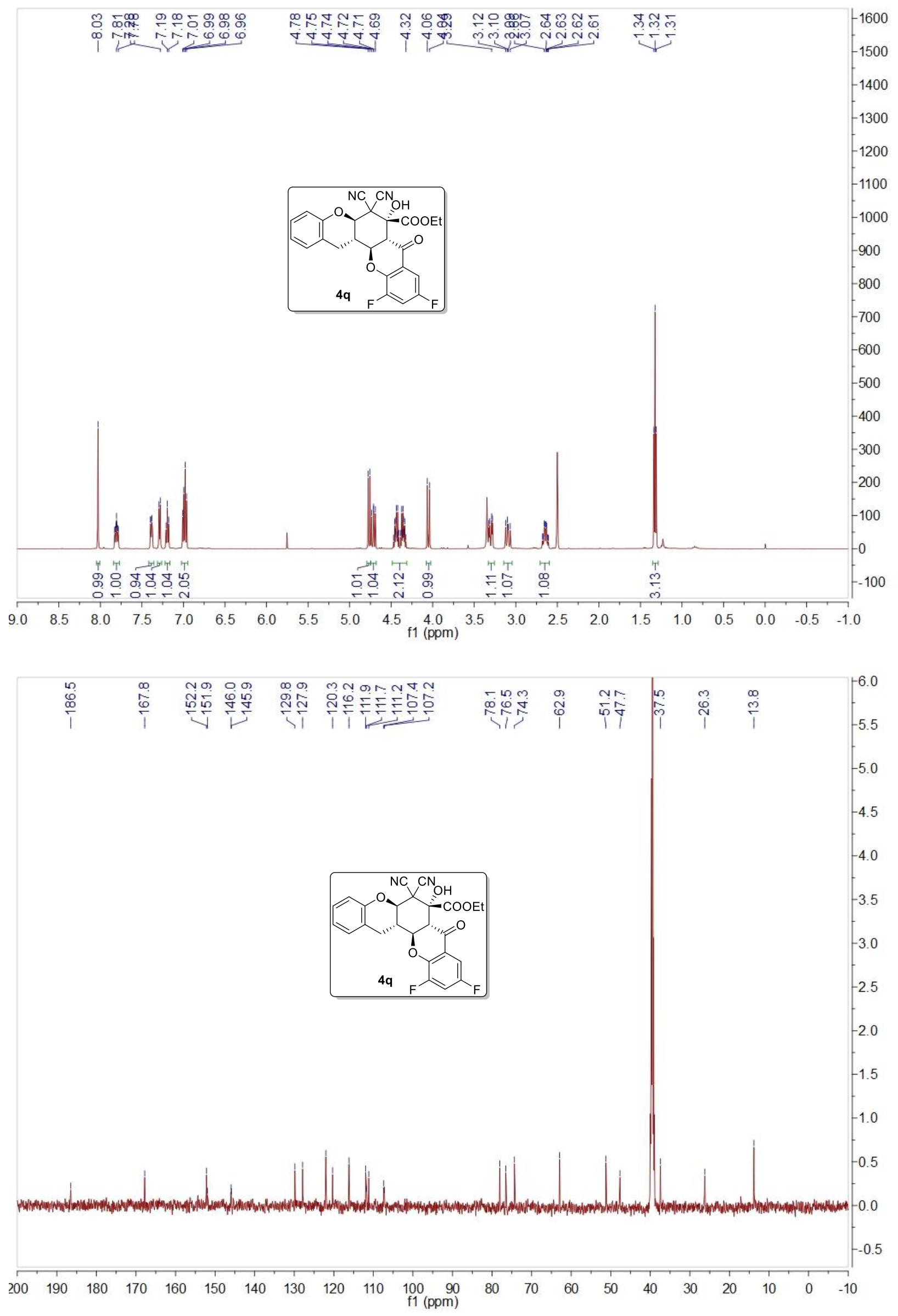
Chrom Type: Fixed WL Chromatogram, $210 \mathrm{~nm}$

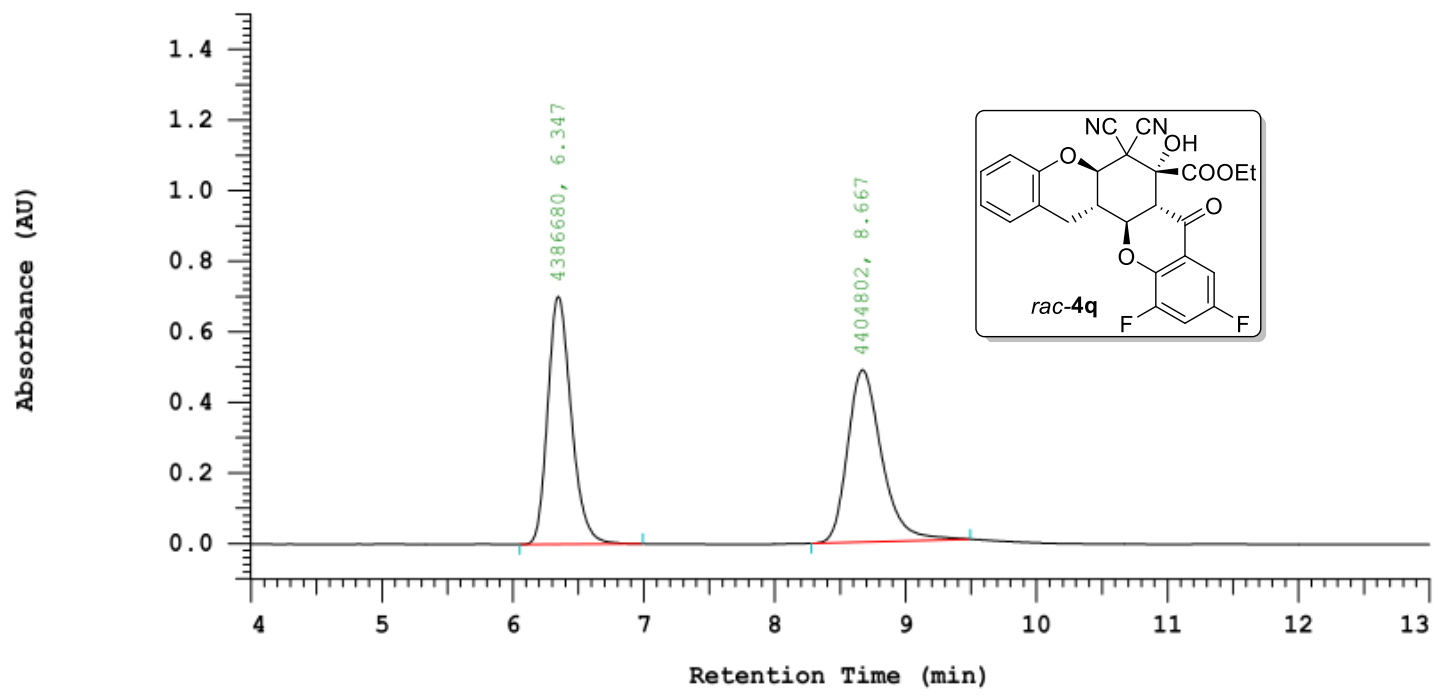

Chrom Type: Fixed WL Chromatogram, $210 \mathrm{~nm}$

Peak Quantitation: AREA

Calculation Method: AREA\%

\begin{tabular}{|c|c|c|c|c|}
\hline No. & RT & Area & Area & $\mathrm{BC}$ \\
\hline 1 & 6.347 & 4386680 & 49.897 & BB \\
\hline 2 & 8.667 & 4404802 & 50.103 & BB \\
\hline & & 8791482 & 100.000 & \\
\hline
\end{tabular}

Chrom Type: Fixed WL Chromatogram, 210 nm

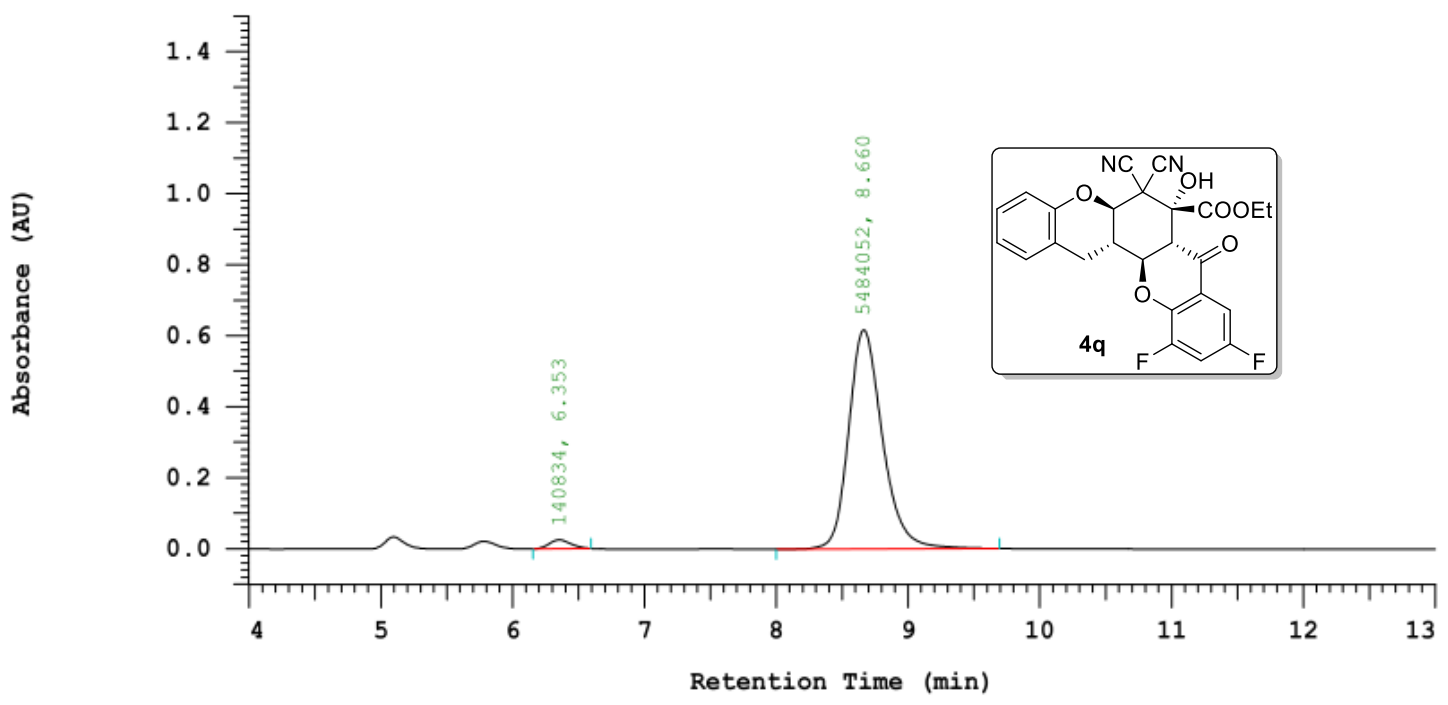

Chrom Type: Fixed WL Chromatogram, $210 \mathrm{~nm}$

Peak Quantitation: AREA

Calculation Method: AREA\%

\begin{tabular}{|c|c|c|c|c|}
\hline No. & $\mathrm{RT}$ & Area & Area $\%$ & $\mathrm{BC}$ \\
\hline 1 & 6.353 & 140834 & 2.504 & $\mathrm{BB}$ \\
\hline 2 & 8.660 & 5484052 & 97.496 & $\mathrm{BB}$ \\
\hline & & 5624886 & 100.000 & \\
\hline
\end{tabular}



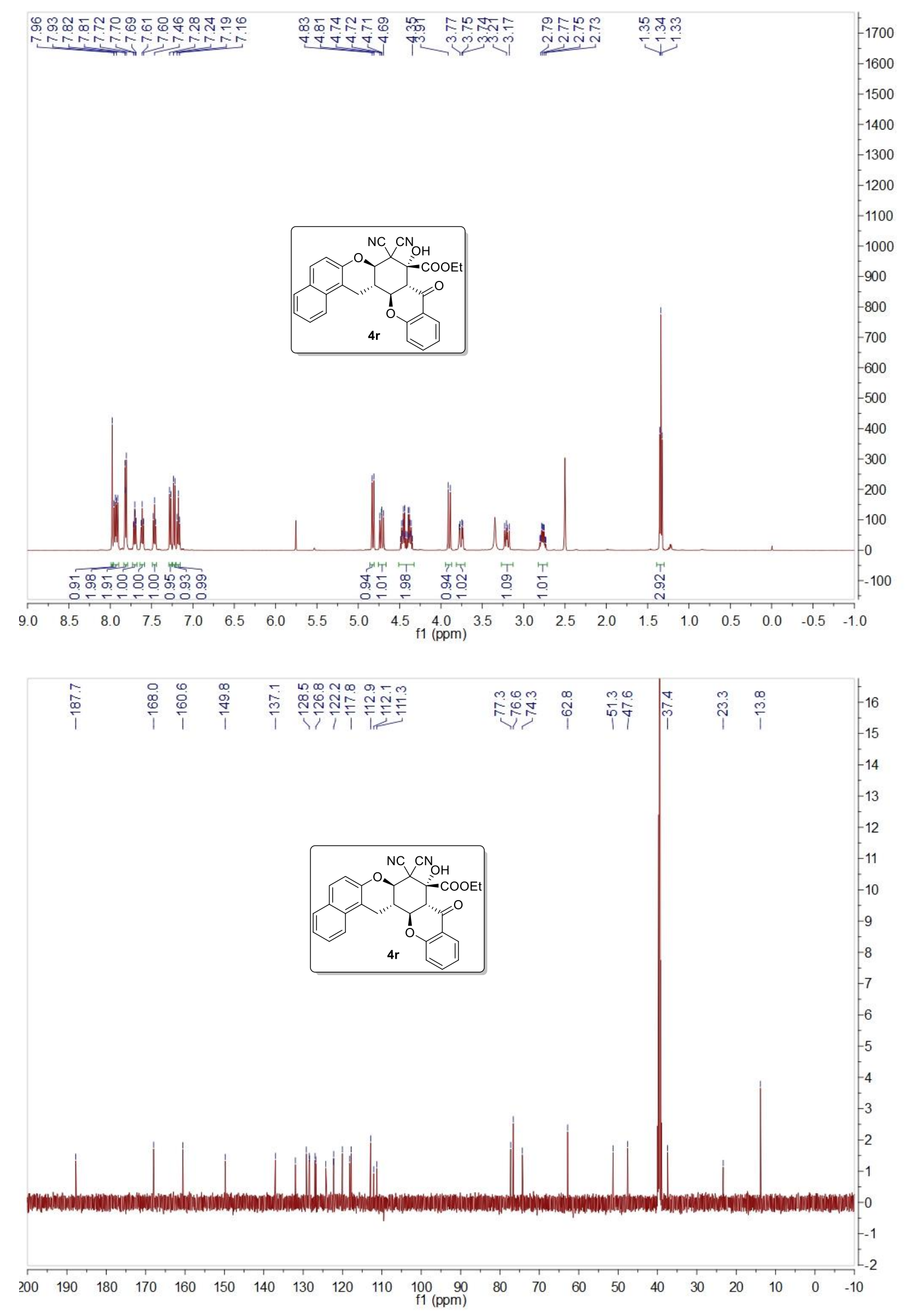
Chrom Type: Fixed WL Chromatogram, 210 nm

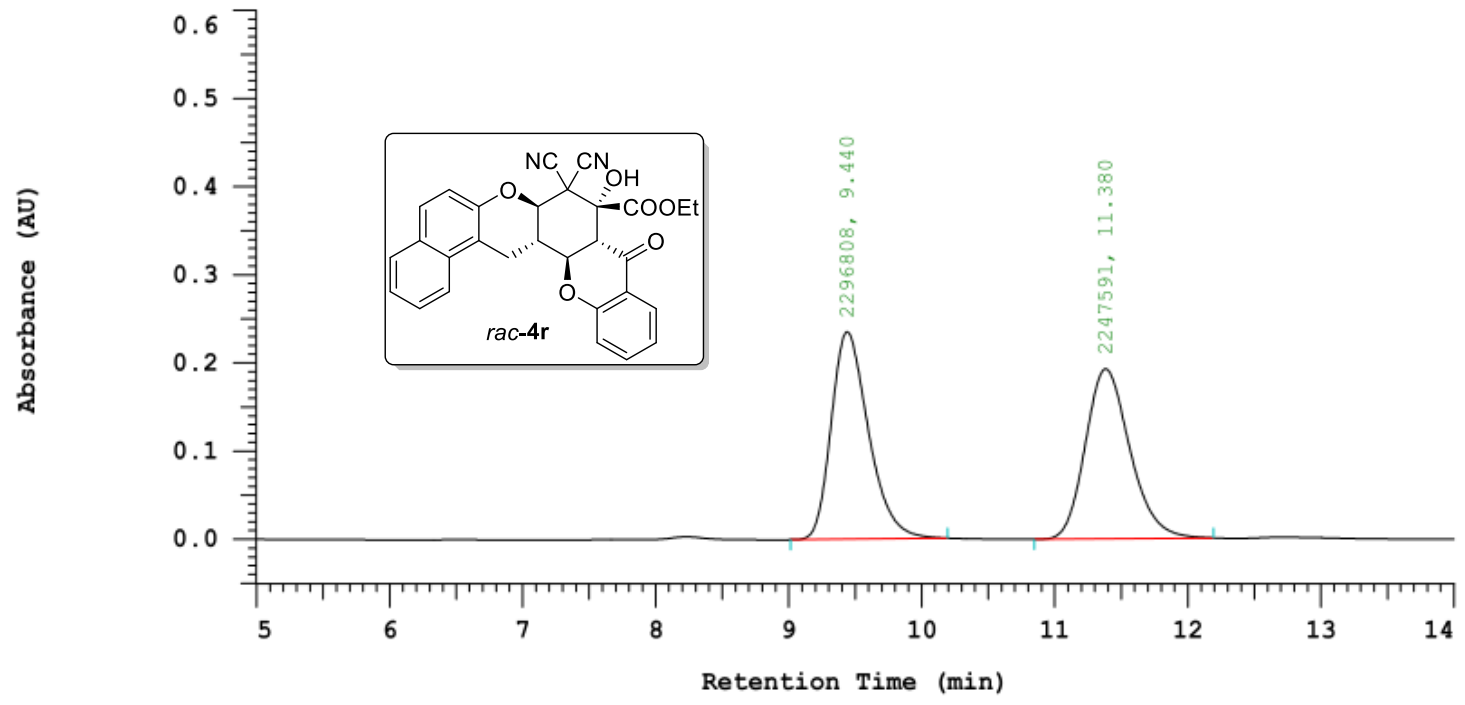

Chrom Type: Fixed WL Chromatogram, $210 \mathrm{~nm}$

Peak Quantitation: AREA

Calculation Method: AREA응

\begin{tabular}{|c|c|c|c|c|}
\hline No. & RT & Area & Area 웅 & $\mathrm{BC}$ \\
\hline 1 & 9.440 & 2296808 & 50.542 & $\mathrm{BB}$ \\
\hline 2 & 11.380 & 2247591 & 49.458 & $\mathrm{BB}$ \\
\hline & & 4544399 & 100.000 & \\
\hline
\end{tabular}

Chrom Type: Fixed WL Chromatogram, $210 \mathrm{~nm}$

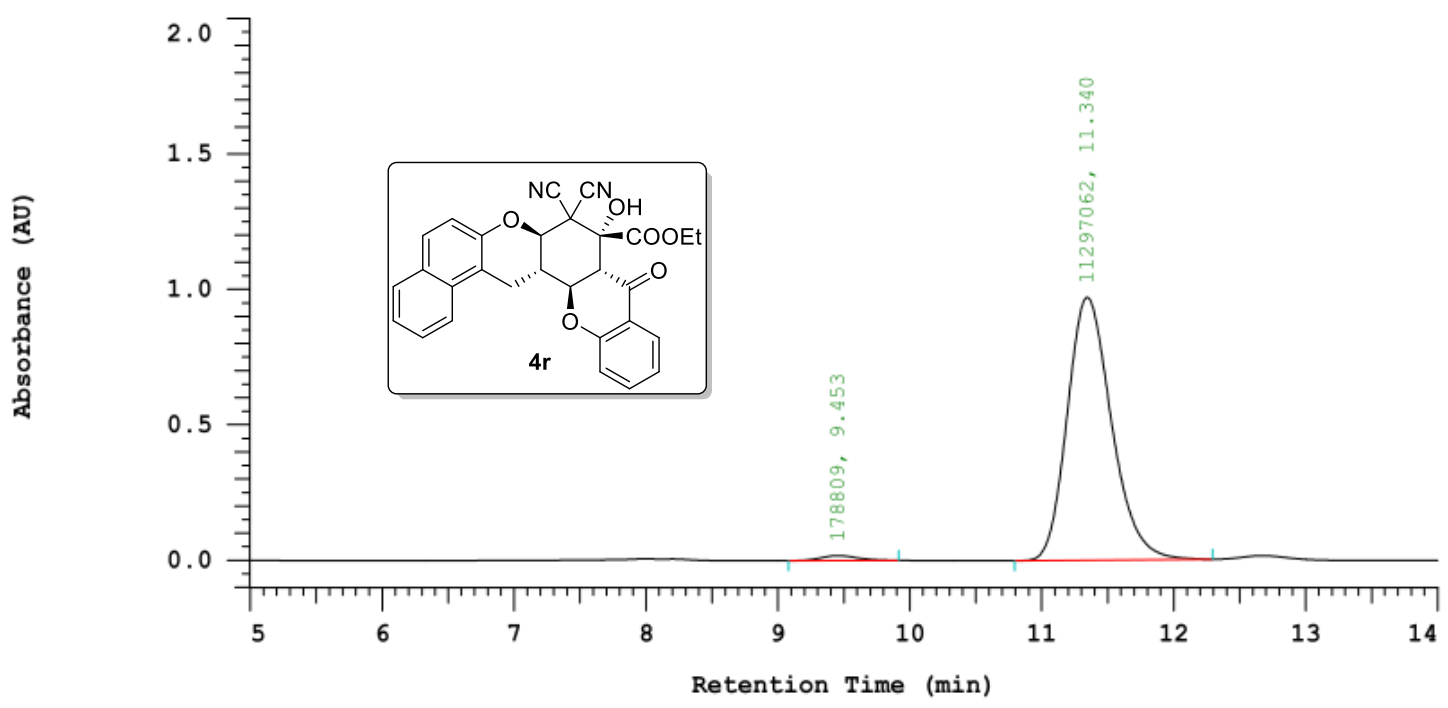

Chrom Type: Fixed WL Chromatogram, $210 \mathrm{~nm}$

Peak Quantitation: AREA

Calculation Method: AREA\%

\begin{tabular}{|c|c|c|c|c|}
\hline No. & $\mathrm{RT}$ & Area & Area $\%$ & $\mathrm{BC}$ \\
\hline 1 & 9.453 & 178809 & 1.558 & $\mathrm{BB}$ \\
\hline 2 & 11.340 & 11297062 & 98.442 & $\mathrm{BB}$ \\
\hline & & 11475871 & 100.000 & \\
\hline
\end{tabular}



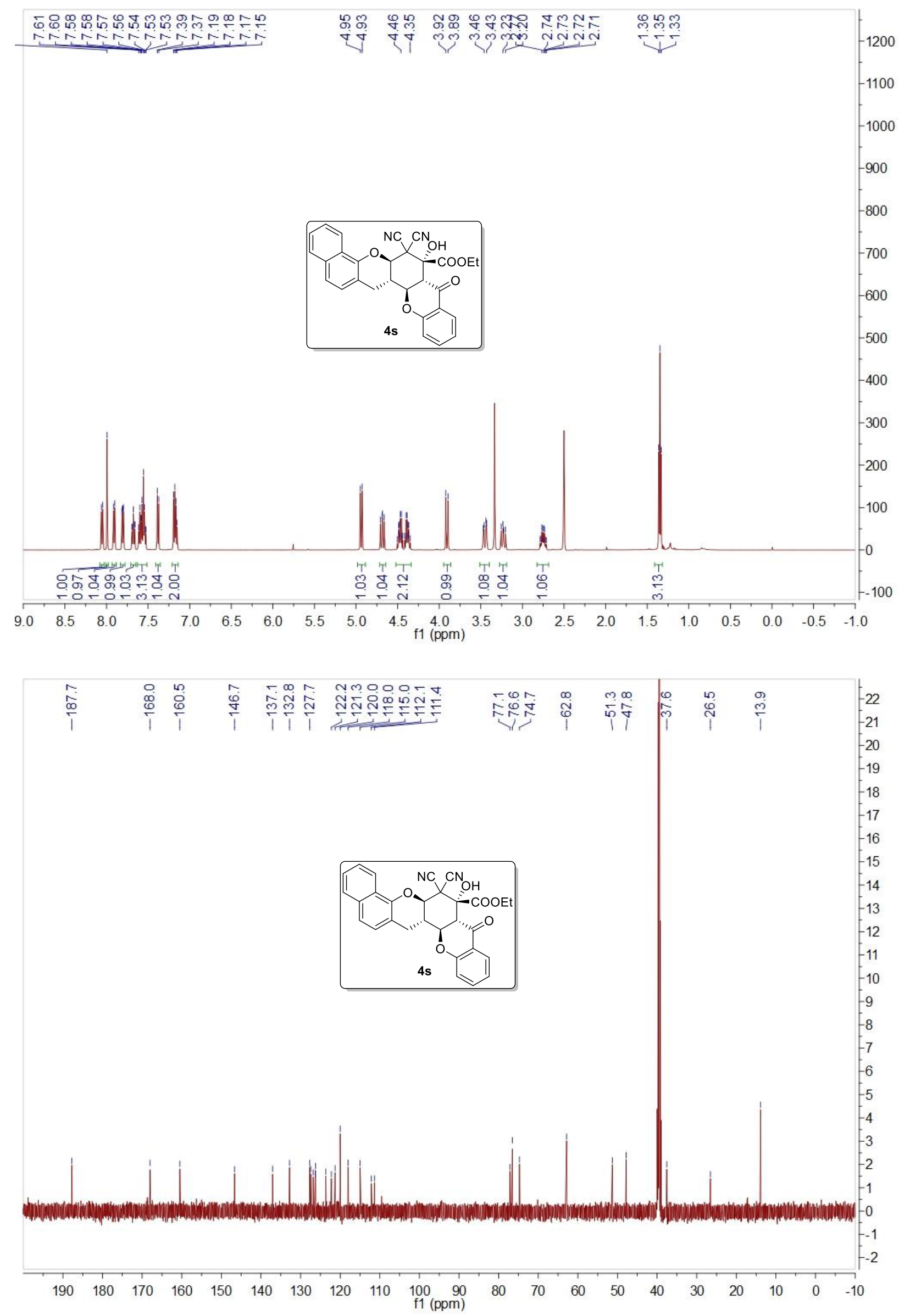
Chrom Type: Fixed WL Chromatogram, 210 nm

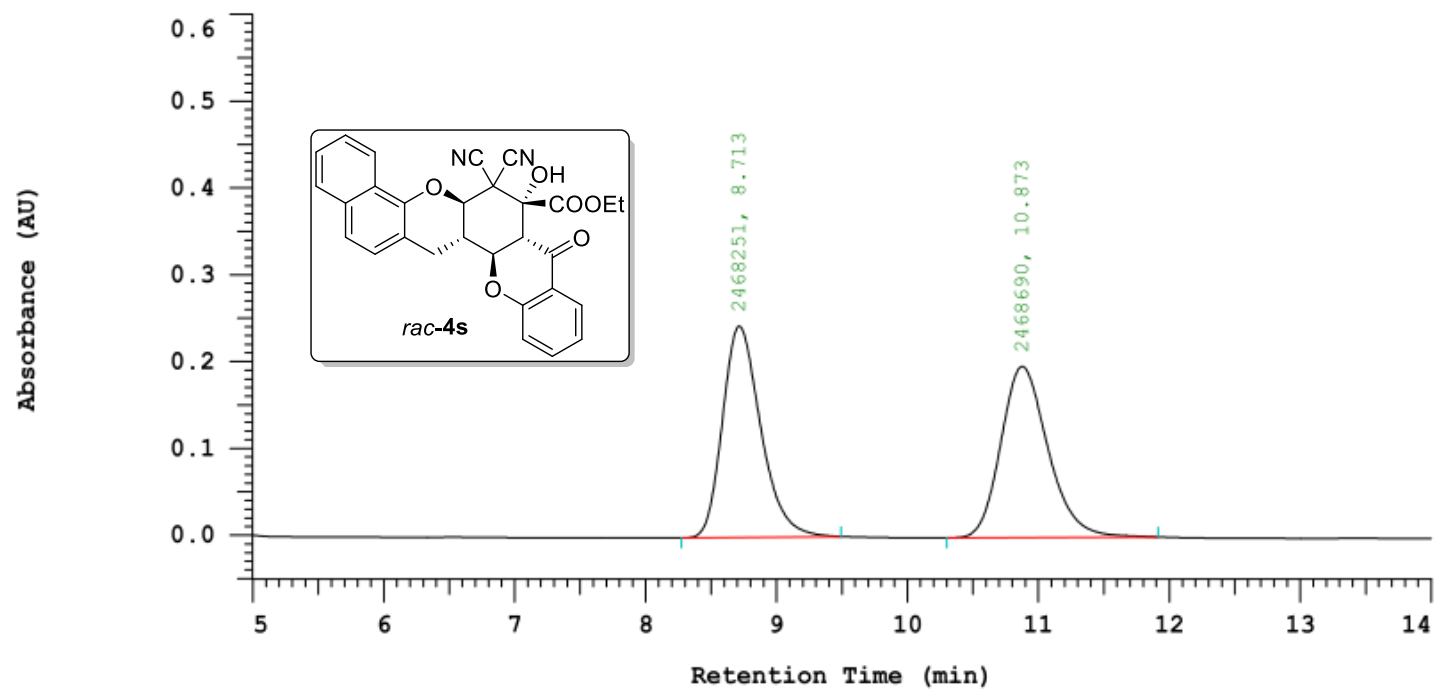

Chrom Type: Fixed WL Chromatogram, $210 \mathrm{~nm}$

Peak Quantitation: AREA

Calculation Method: AREA\%

\begin{tabular}{|c|c|c|c|c|}
\hline No. & RT & Area & Area 응 & $\mathrm{BC}$ \\
\hline 1 & 8.713 & 2468251 & 49.996 & $\mathrm{BB}$ \\
\hline 2 & 10.873 & 2468690 & 50.004 & $\mathrm{BB}$ \\
\hline & & 4936941 & 100.000 & \\
\hline
\end{tabular}

Chrom Type: Fixed WL Chromatogram, 210 nm

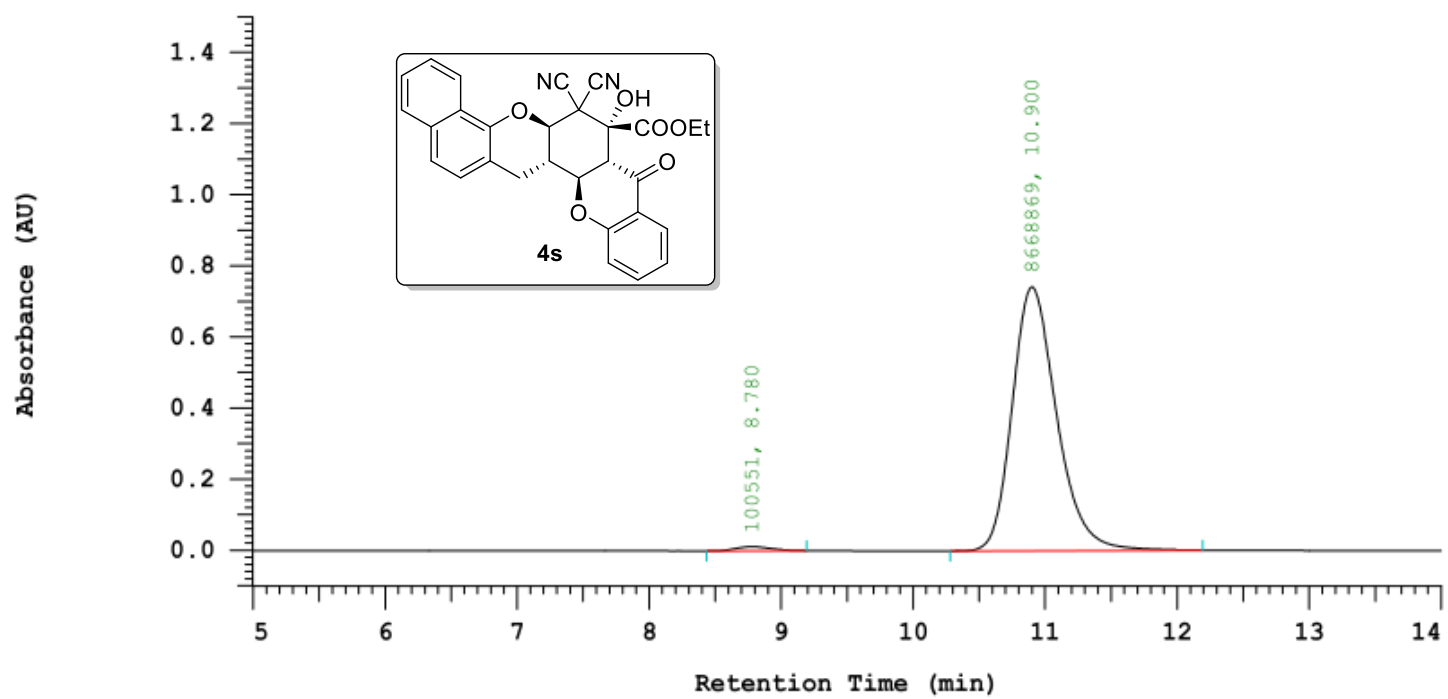

Chrom Type: Fixed WL Chromatogram, $210 \mathrm{~nm}$

Peak Quantitation: AREA

Calculation Method: AREA응

\begin{tabular}{|c|c|c|c|c|}
\hline No. & RT & Area & Area $\frac{\circ}{\partial}$ & $\mathrm{BC}$ \\
\hline 1 & 8.780 & 100551 & 1.147 & $\mathrm{BB}$ \\
\hline \multirow[t]{2}{*}{2} & 10.900 & 8668869 & 98.853 & $\mathrm{BB}$ \\
\hline & & 8769420 & 100.000 & \\
\hline
\end{tabular}



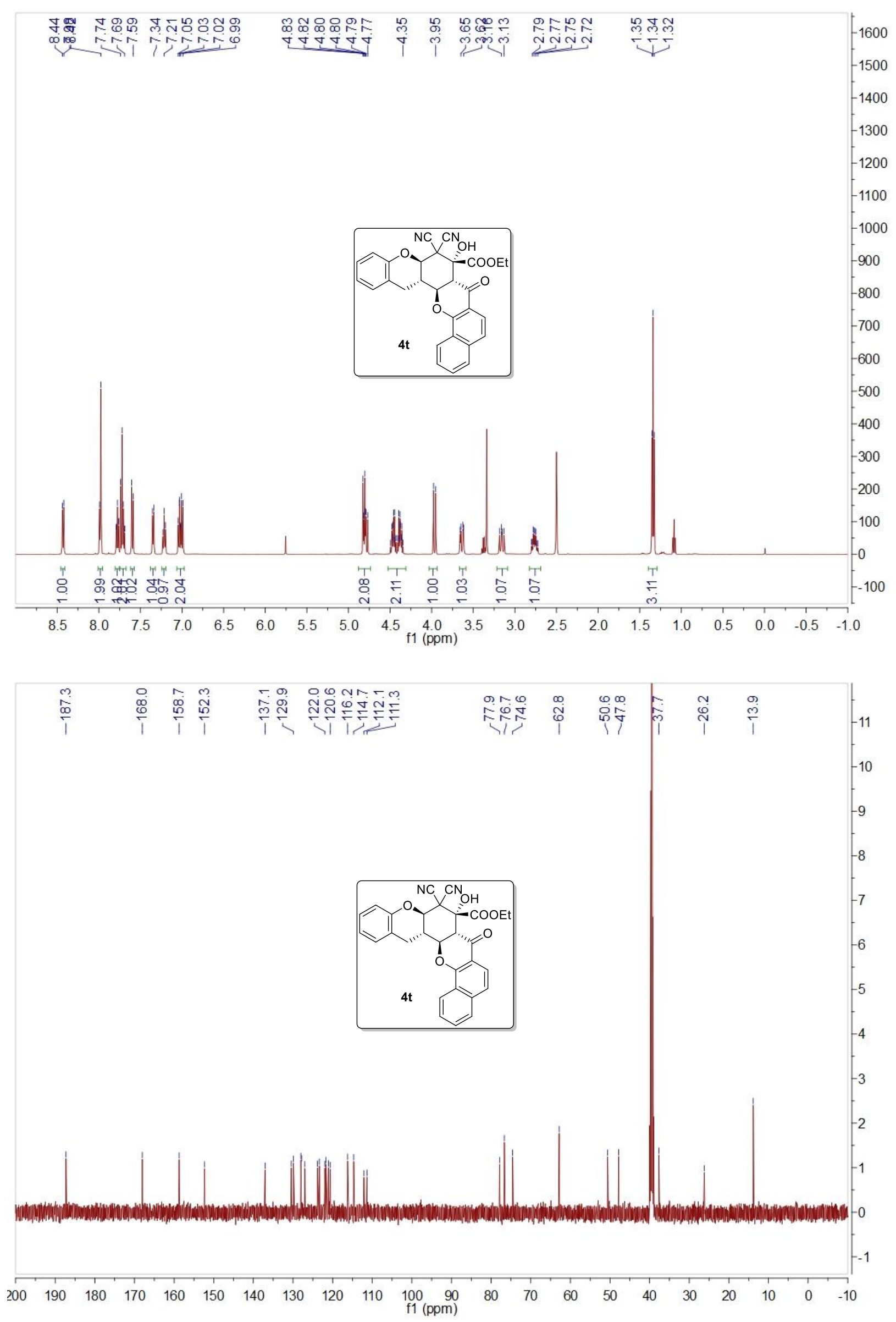
Chrom Type: Fixed WL Chromatogram, 210 nm

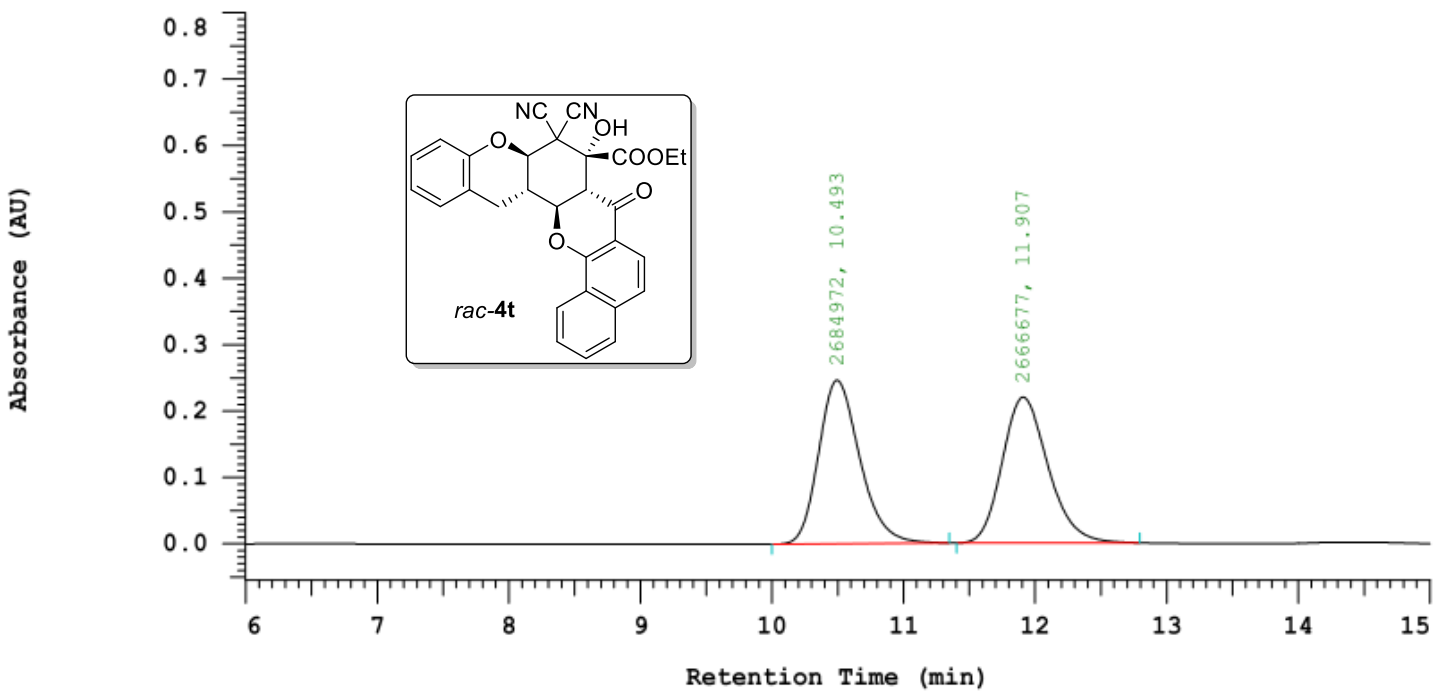

Chrom Type: Fixed WL Chromatogram, $210 \mathrm{~nm}$

Peak Quantitation: AREA

Calculation Method: AREA응

\begin{tabular}{ccccc} 
No. & RT & Area & Area & BC \\
\hline 1 & 10.493 & 2684972 & 50.171 & BB \\
2 & 11.907 & 2666677 & 49.829 & BB \\
\hline & & 5351649 & 100.000 & \\
\hline
\end{tabular}

Chrom Type: Fixed WL Chromatogram, 210 nm

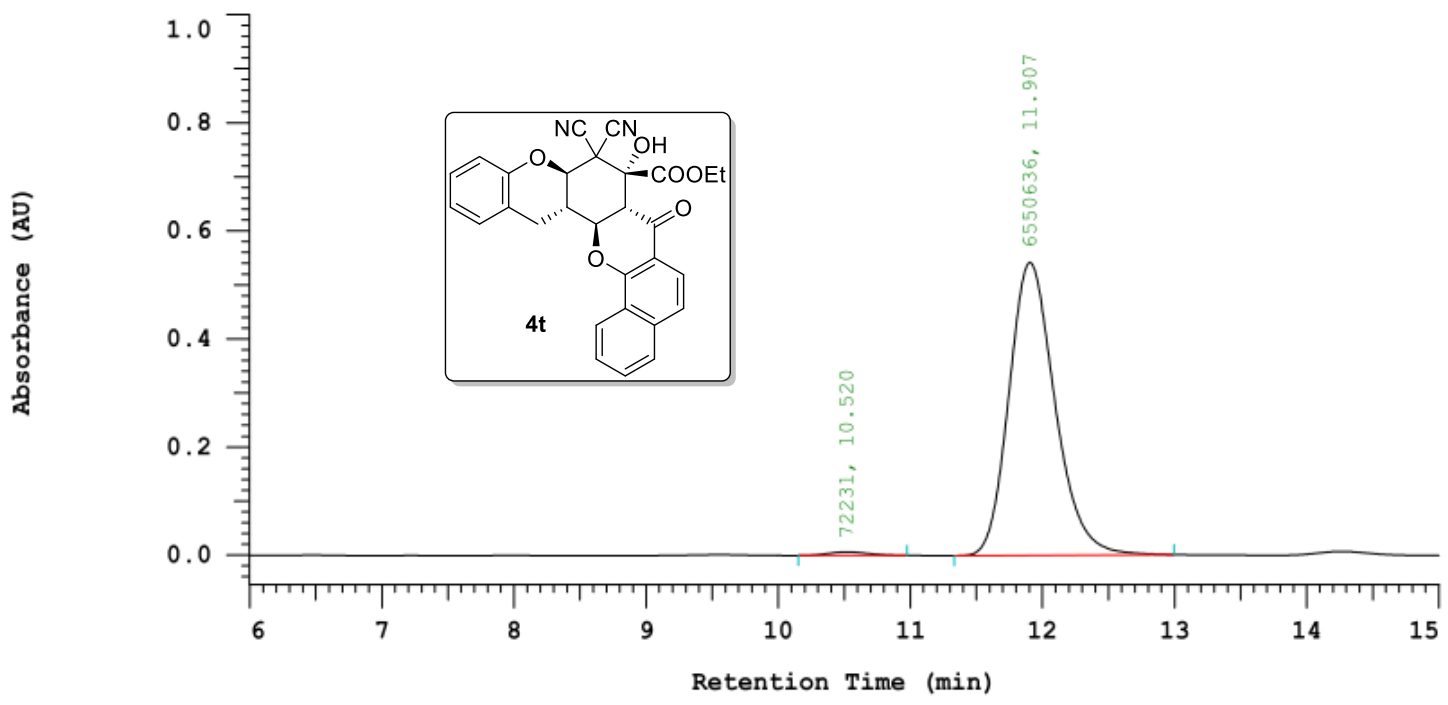

Chrom Type: Fixed WL Chromatogram, $210 \mathrm{~nm}$

Peak Quantitation: AREA

Calculation Method: AREA\%

\begin{tabular}{|c|c|c|c|c|}
\hline No. & $\mathrm{RT}$ & Area & Area & $\mathrm{BC}$ \\
\hline 1 & 10.520 & 72231 & 1.091 & BB \\
\hline 2 & 11.907 & 6550636 & 98.909 & BB \\
\hline & & 6622867 & 100.000 & \\
\hline
\end{tabular}



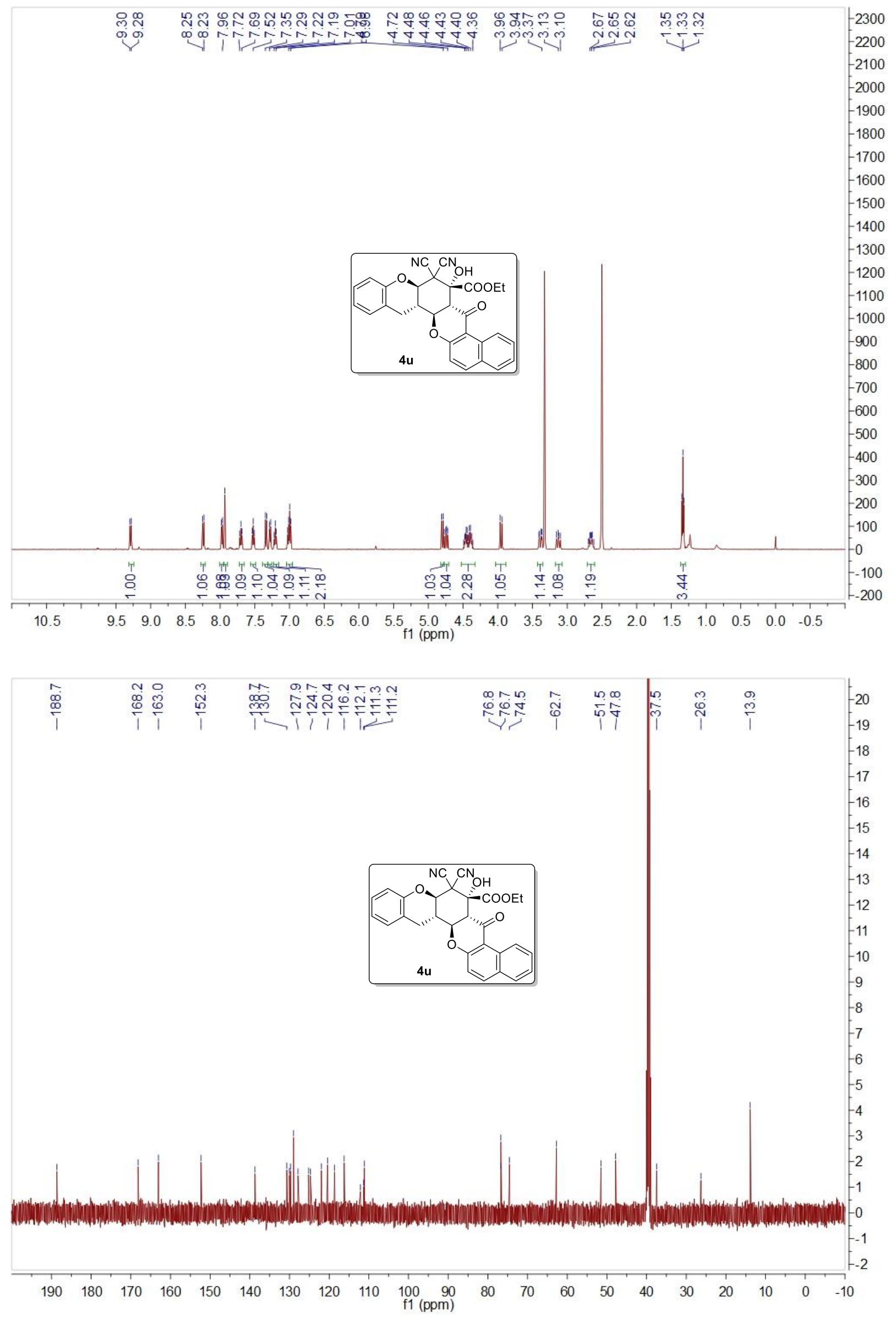
Chrom Type: Fixed WL Chromatogram, 210 nm

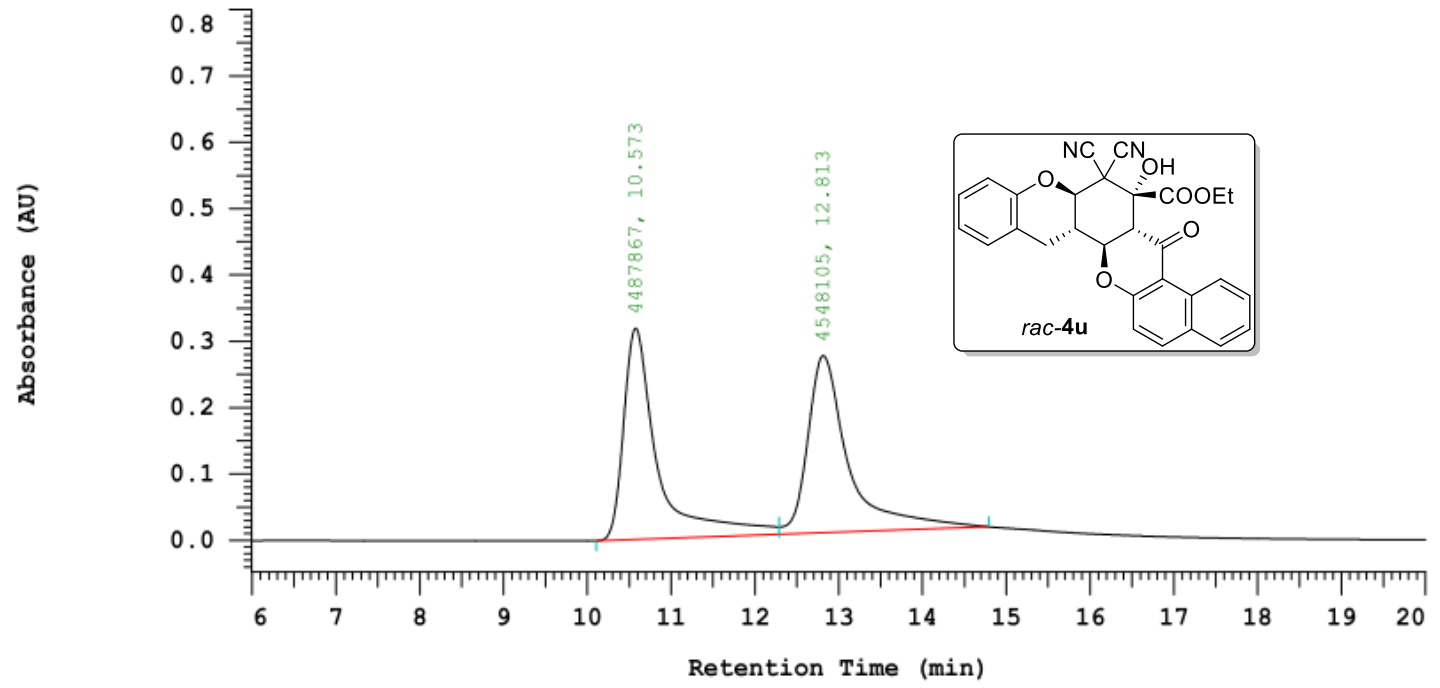

Chrom Type: Fixed WL Chromatogram, $210 \mathrm{~nm}$

Peak Quantitation: AREA

Calculation Method: AREA\%

\begin{tabular}{ccccc} 
No. & RT & Area & Area & BC \\
\hline 1 & 10.573 & 4487867 & 49.667 & BV \\
2 & 12.813 & 4548105 & 50.333 & VB \\
\hline & & 9035972 & 100.000 & \\
\hline
\end{tabular}

Chrom Type: Fixed WL Chromatogram, 210 nm

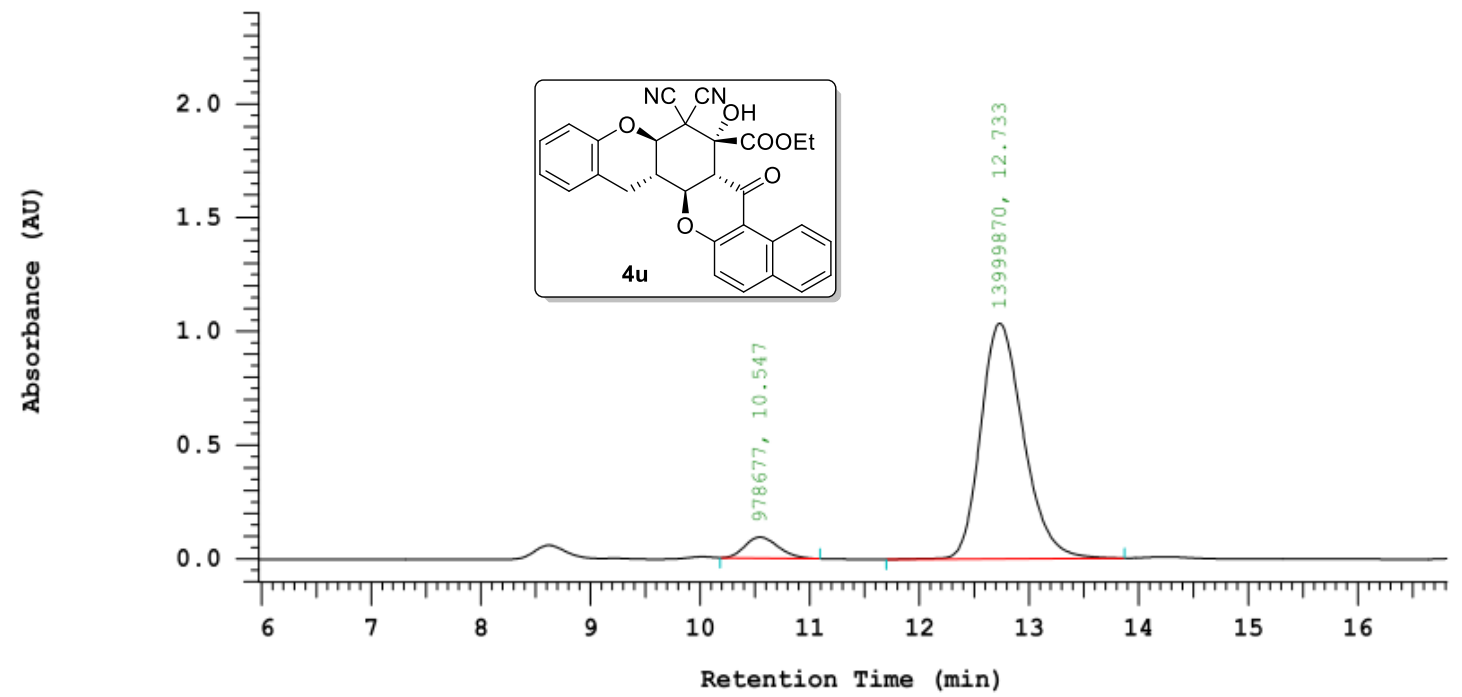

Chrom Type: Fixed WL Chromatogram, $210 \mathrm{~nm}$

Peak Quantitation: AREA

Calculation Method: AREA응

\begin{tabular}{|c|c|c|c|c|}
\hline No. & RT & Area & Area $\%$ & $\mathrm{BC}$ \\
\hline 1 & 10.547 & 978677 & 6.534 & BB \\
\hline \multirow[t]{2}{*}{2} & 12.733 & 13999870 & 93.466 & $\mathrm{BB}$ \\
\hline & & 14978547 & 100.000 & \\
\hline
\end{tabular}



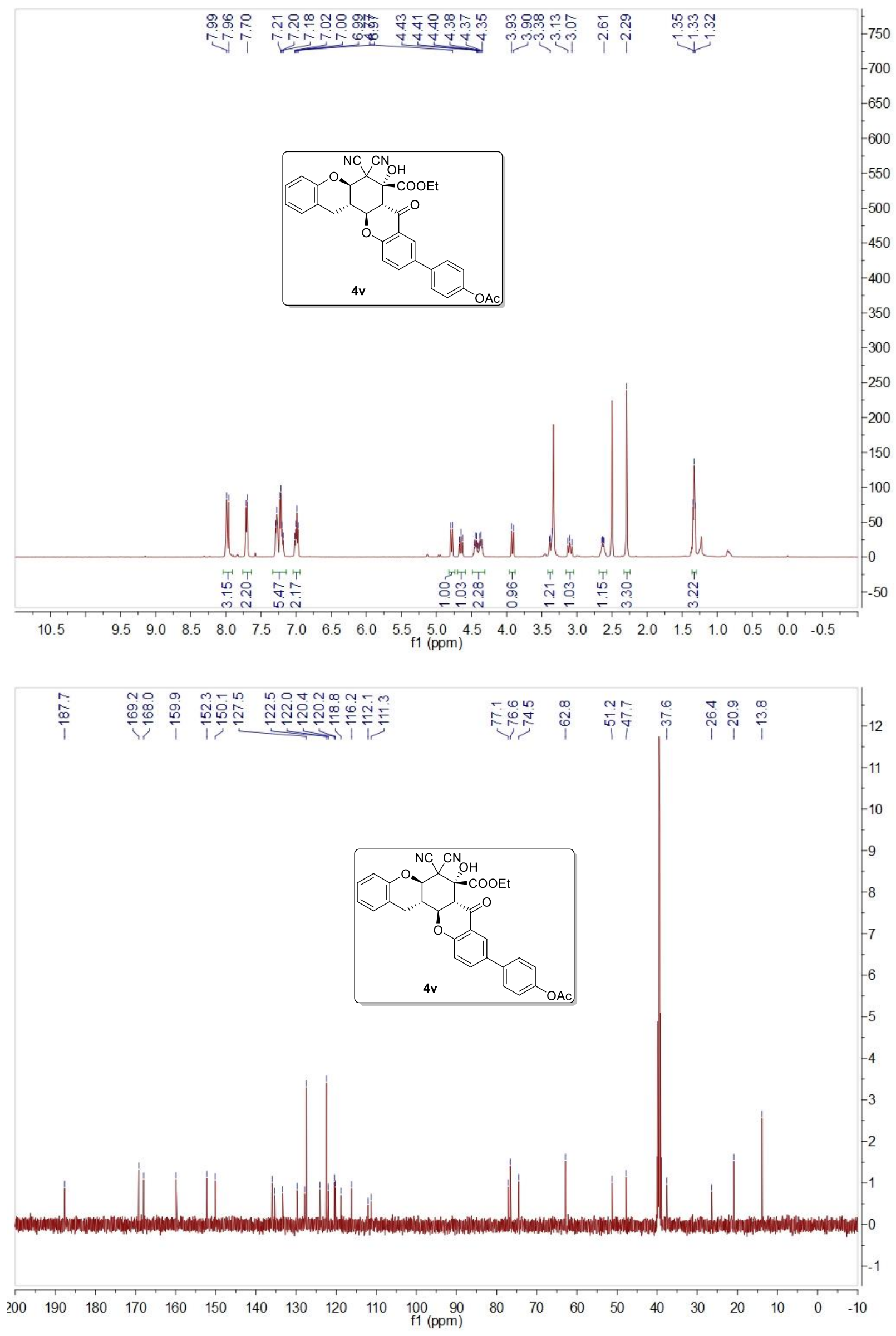
Chrom Type: Fixed WL Chromatogram, 210 nm

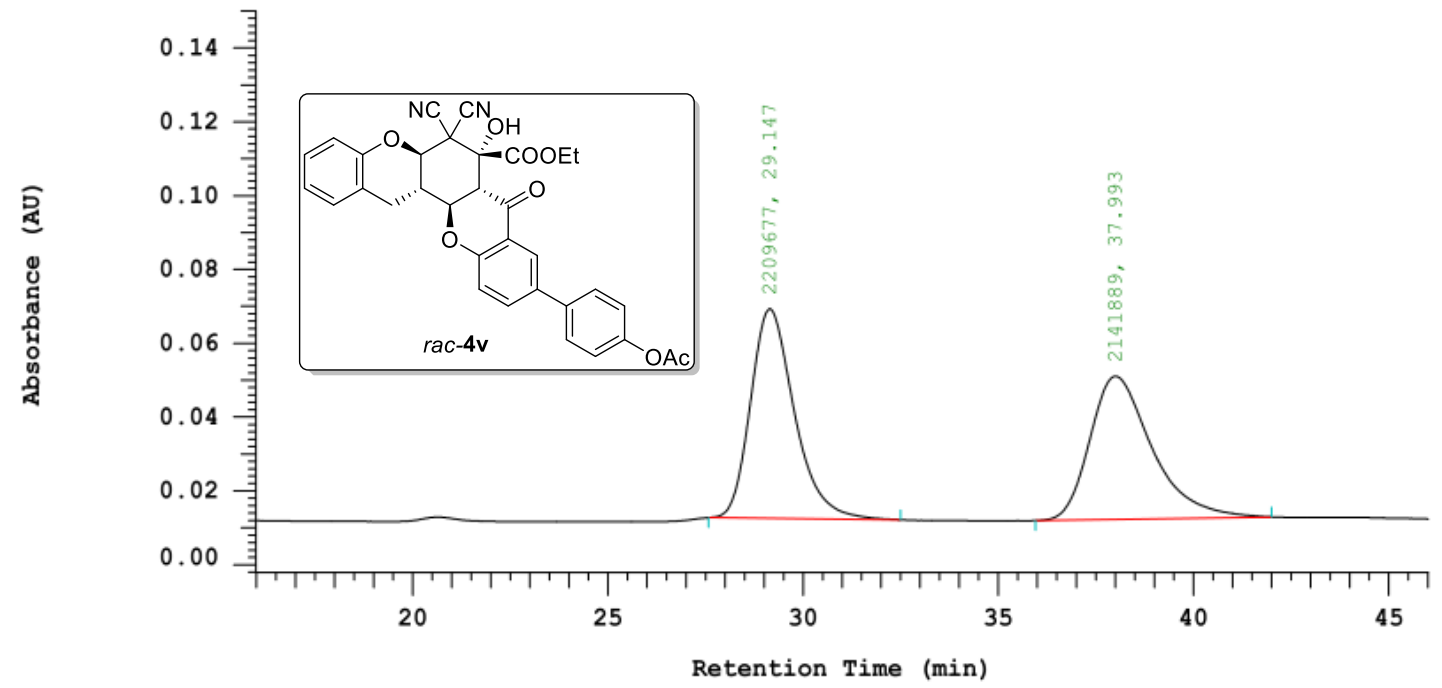

Chrom Type: Fixed WL Chromatogram, $210 \mathrm{~nm}$

Peak Quantitation: AREA

Calculation Method: AREA\%

\begin{tabular}{ccccc} 
No. & RT & Area & Area $\%$ & BC \\
\hline 1 & 29.147 & 2209677 & 50.779 & BB \\
2 & 37.993 & 2141889 & 49.221 & BB \\
\hline & 4351566 & 100.000 & \\
\hline
\end{tabular}

Chrom Type: Fixed WL Chromatogram, 210 nm

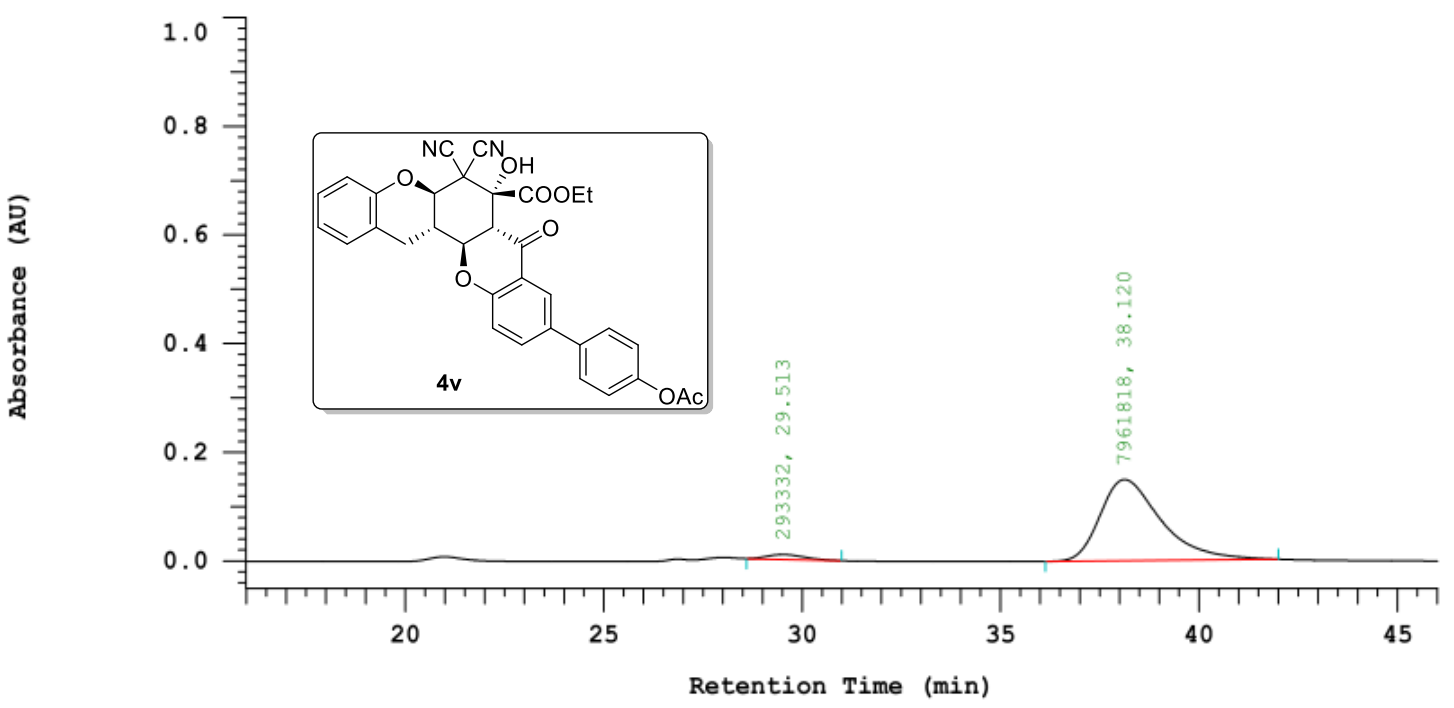

Peak Quantitation: AREA

Chrom Type: Fixed WL Chromatogram, $210 \mathrm{~nm}$ Calculation Method: AREA:

\begin{tabular}{|c|c|c|c|c|}
\hline No. & $\mathrm{RT}$ & Area & Area 응 & $\mathrm{BC}$ \\
\hline 1 & 29.513 & 293332 & 3.553 & $\mathrm{BB}$ \\
\hline \multirow[t]{2}{*}{2} & 38.120 & 7961818 & 96.447 & $\mathrm{BB}$ \\
\hline & & 8255150 & 100.000 & \\
\hline
\end{tabular}



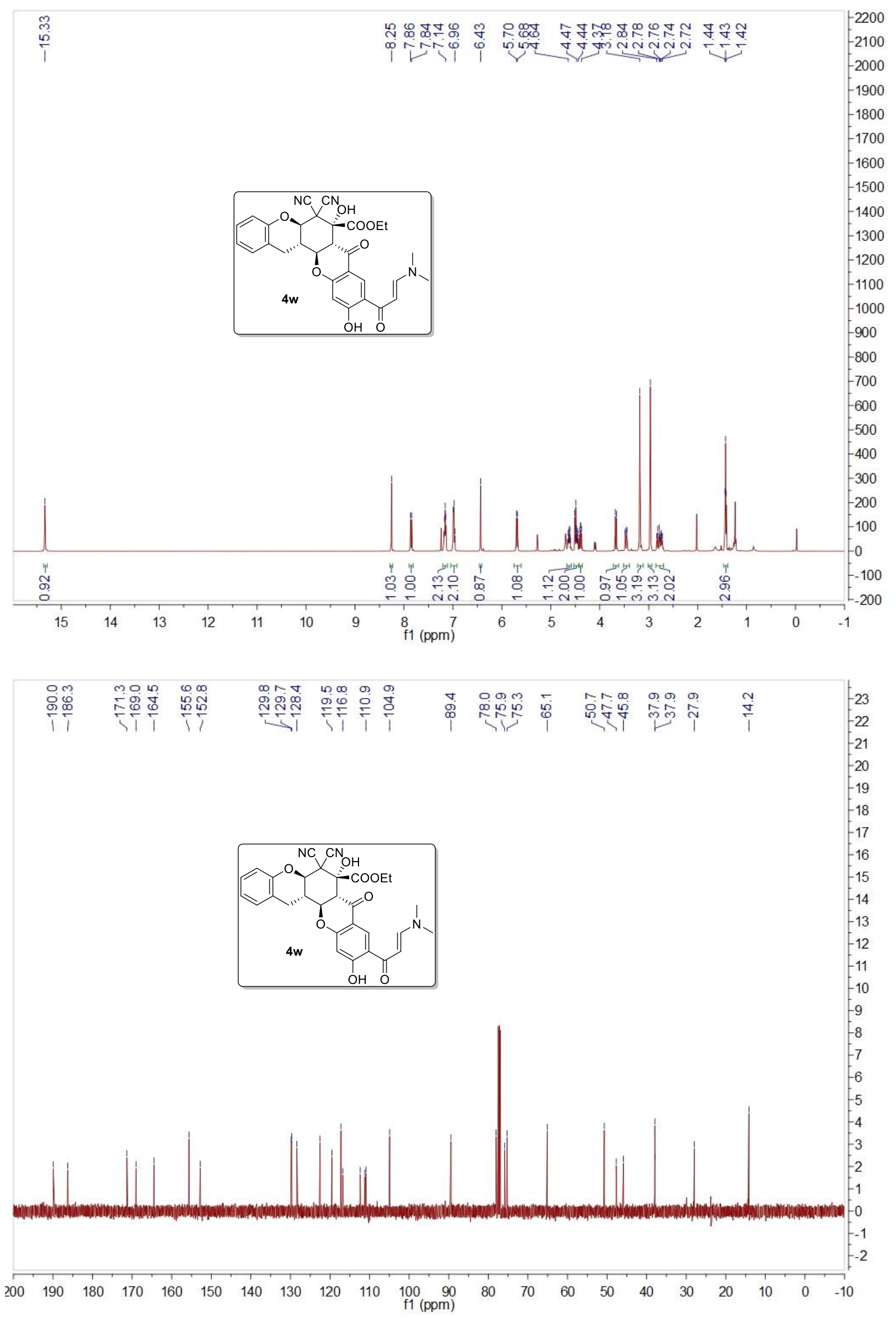
Chrom Type: Fixed WL Chromatogram, 260 nm

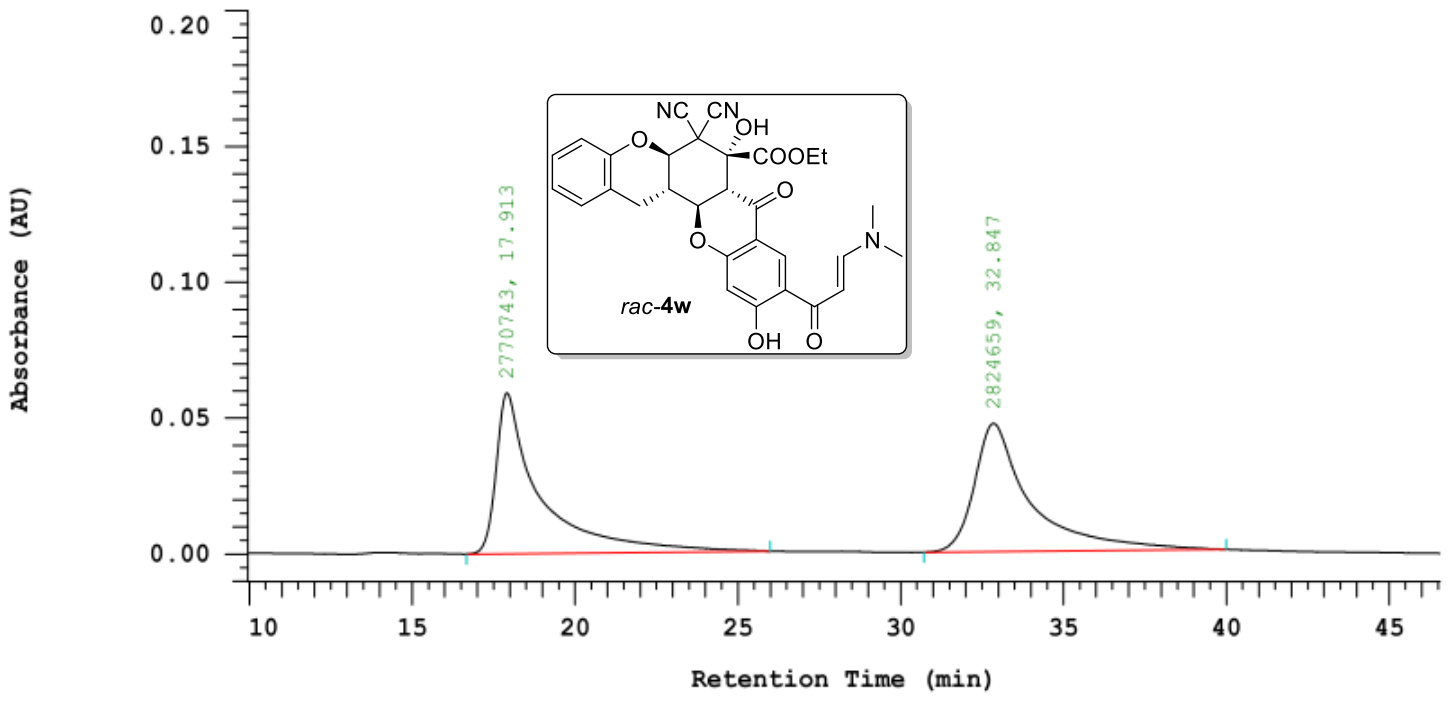

Chrom Type: Fixed WL Chromatogram, $260 \mathrm{~nm}$

Peak Quantitation: AREA

Calculation Method: AREA\%

\begin{tabular}{ccccc} 
No. & RT & Area & Area & BC \\
\hline 1 & 17.913 & 2770743 & 49.518 & BB \\
2 & 32.847 & 2824659 & 50.482 & BB \\
\hline & & 5595402 & 100.000 & \\
\hline
\end{tabular}

Chrom Type: Fixed WL Chromatogram, 260 nm

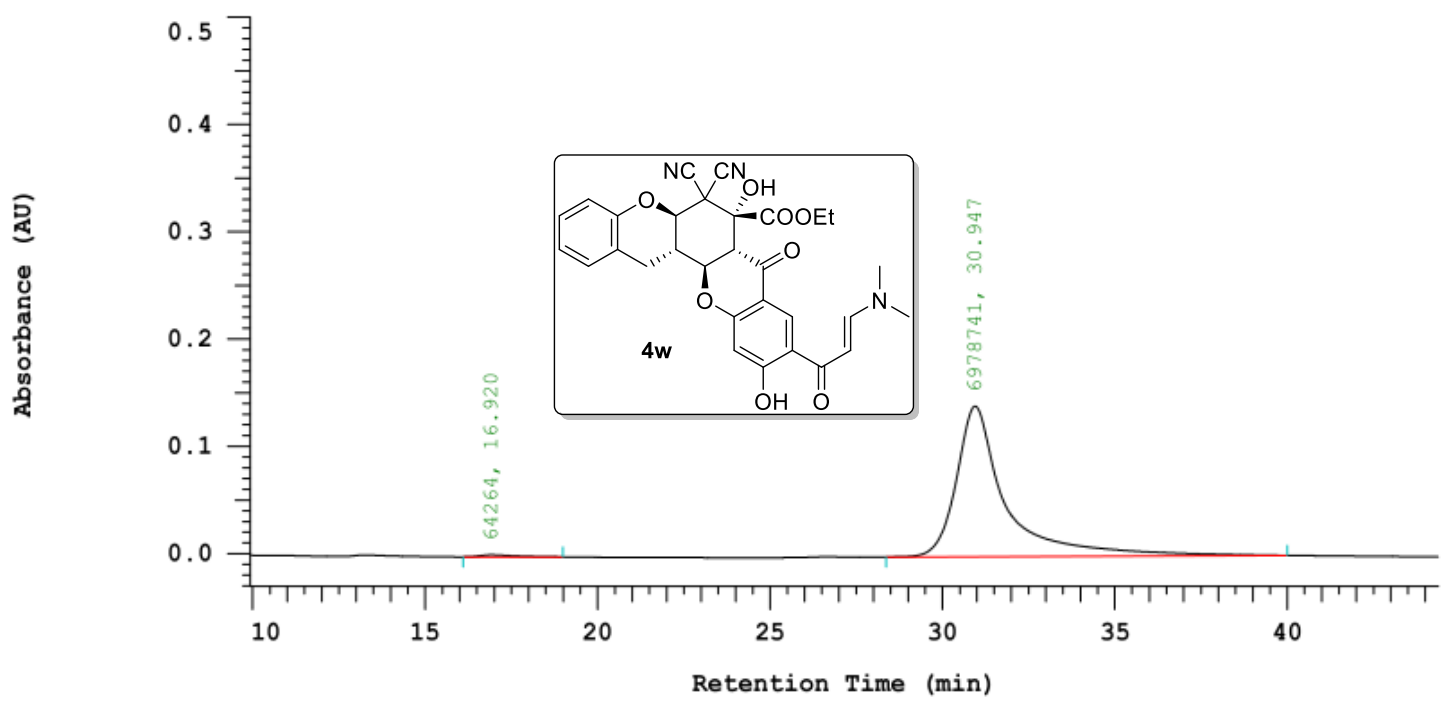

Chrom Type: Fixed WL Chromatogram, $260 \mathrm{~nm}$

Peak Quantitation: AREA

Calculation Method: AREA\%

\begin{tabular}{|c|c|c|c|c|}
\hline No. & $\mathrm{RT}$ & Area & Area \% & $\mathrm{BC}$ \\
\hline 1 & 16.920 & 64264 & 0.912 & $\mathrm{BB}$ \\
\hline \multirow[t]{2}{*}{2} & 30.947 & 6978741 & 99.088 & $\mathrm{BB}$ \\
\hline & & 7043005 & 100.000 & \\
\hline
\end{tabular}



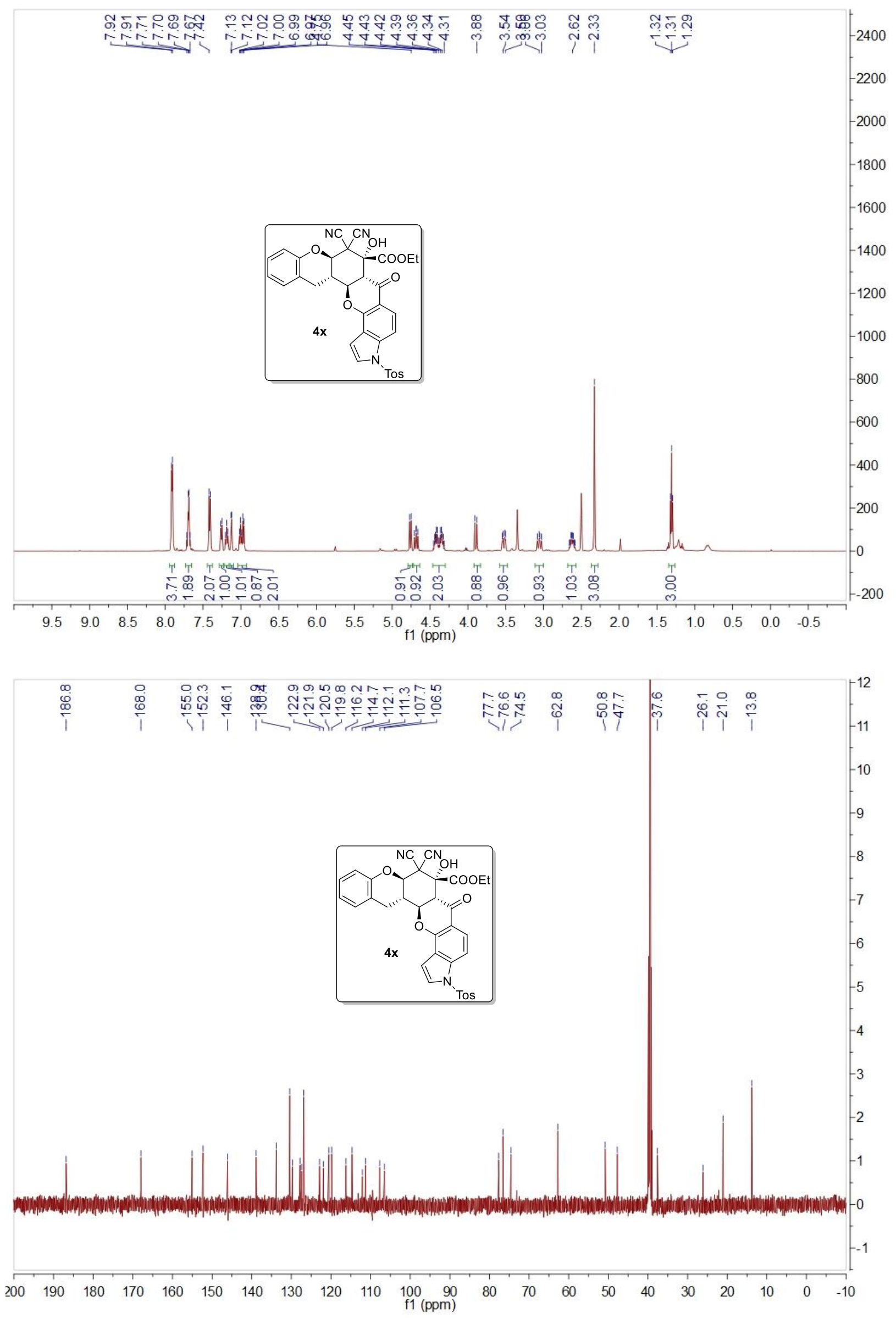
Chrom Type: Fixed WL Chromatogram, 230 nm

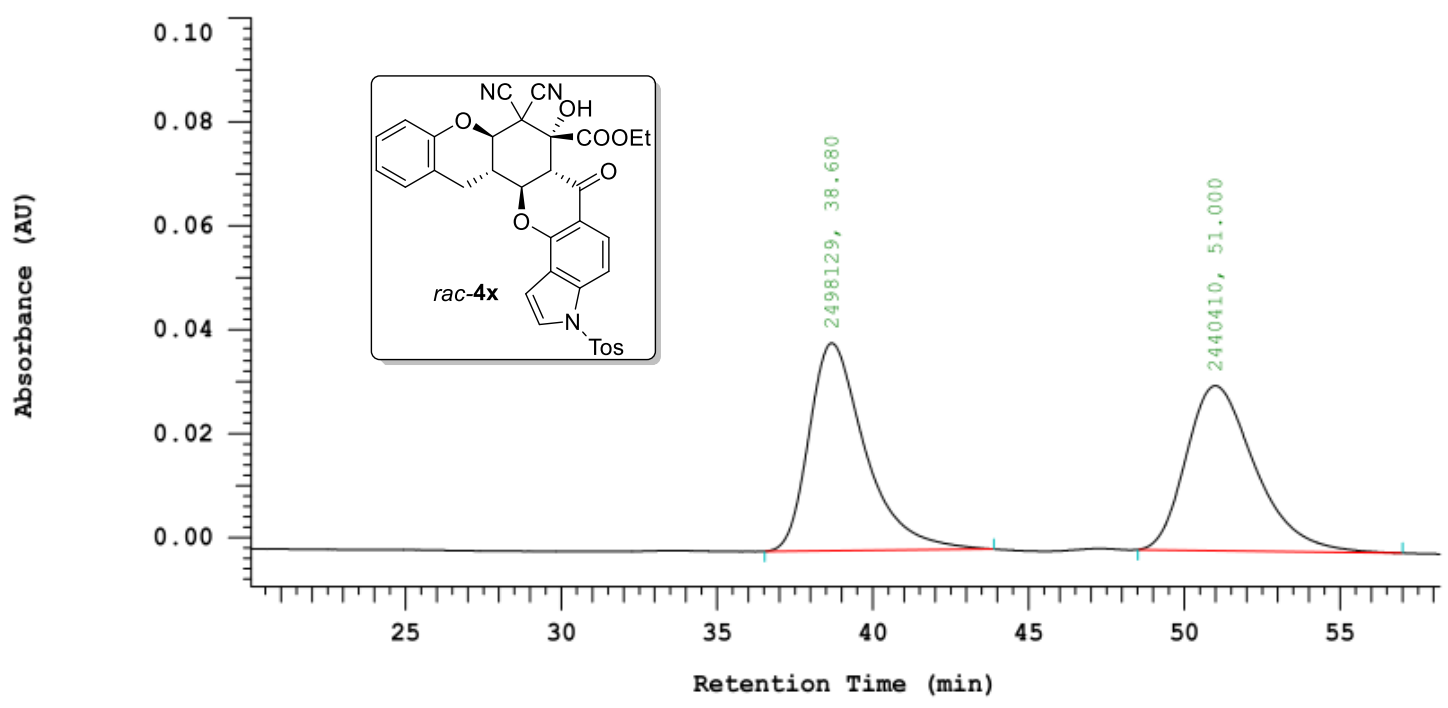

Chrom Type: Fixed WL Chromatogram, $230 \mathrm{~nm}$

Peak Quantitation: AREA

Calculation Method: AREA\%

\begin{tabular}{ccccc} 
No. & RT & Area & Area & BC \\
\hline 1 & 38.680 & 2498129 & 50.584 & BB \\
2 & 51.000 & 2440410 & 49.416 & BB \\
\hline & & 4938539 & 100.000 & \\
\hline
\end{tabular}

Chrom Type: Fixed WL Chromatogram, 230 nm

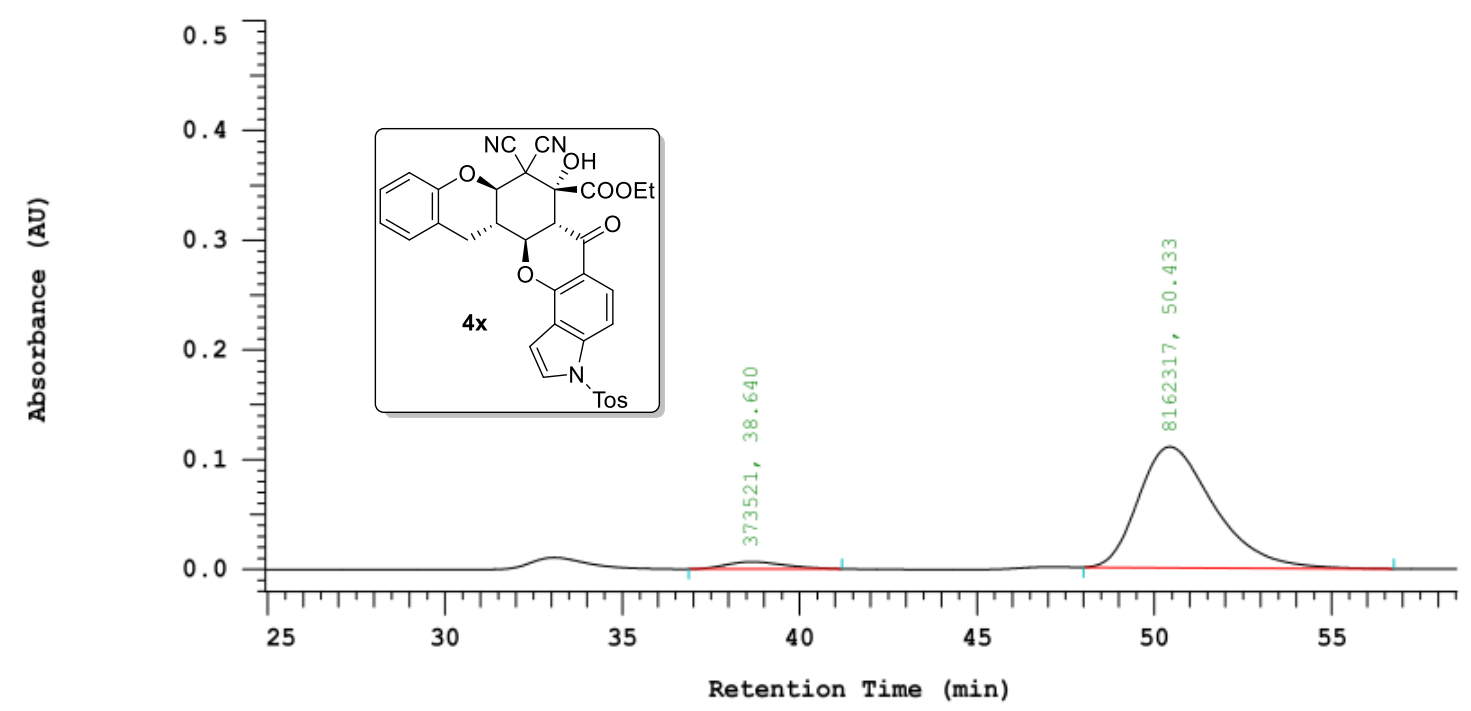

Peak Quantitation: AREA

Calculation Method: AREA\%

\begin{tabular}{|c|c|c|c|c|}
\hline No. & $\mathrm{RT}$ & Area & Area $\%$ & $\mathrm{BC}$ \\
\hline 1 & 38.640 & 373521 & 4.376 & BB \\
\hline \multirow[t]{2}{*}{2} & 50.433 & 8162317 & 95.624 & BB \\
\hline & & 8535838 & 100.000 & \\
\hline
\end{tabular}



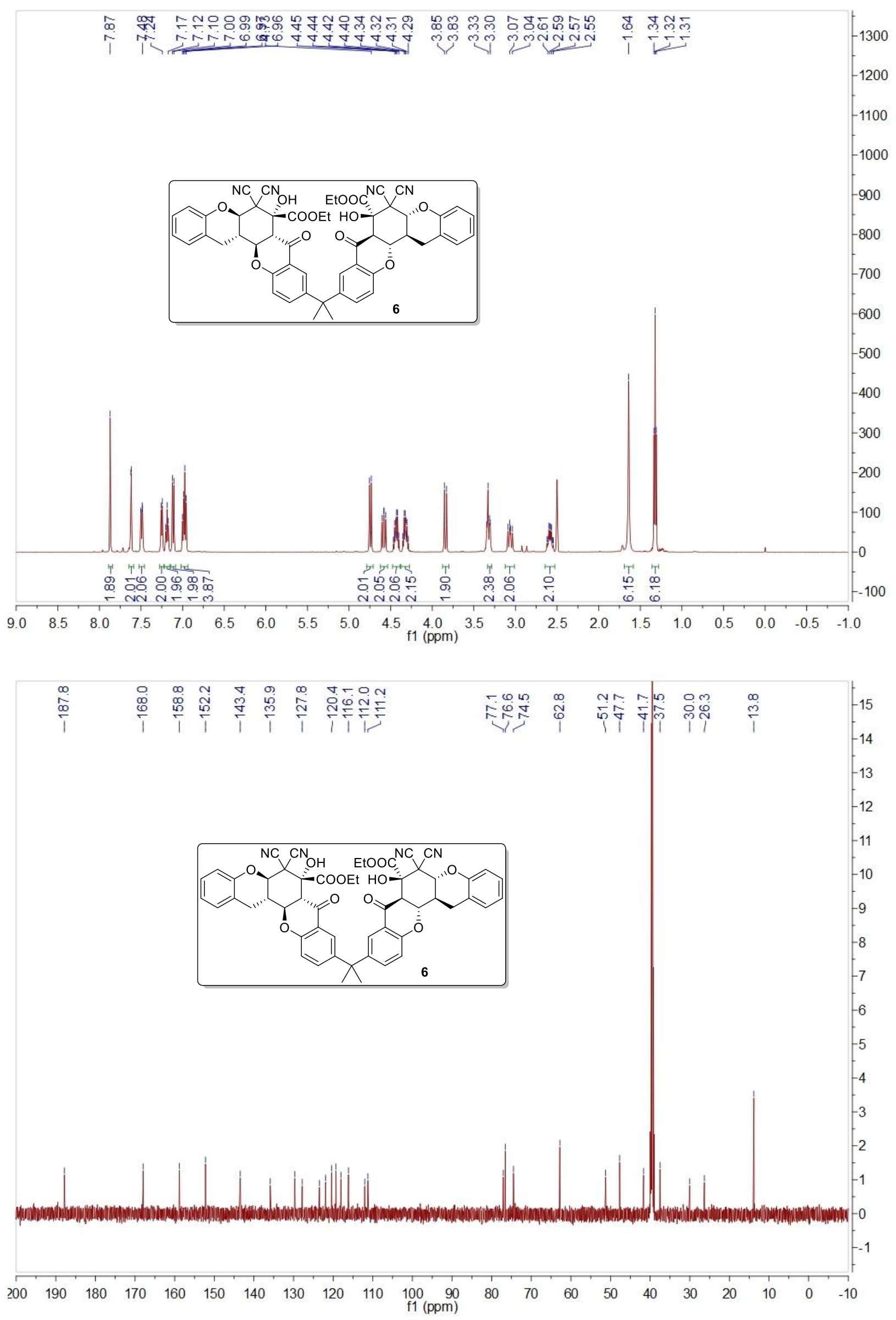
Chrom Type: Fixed WL Chromatogram, 230 nm

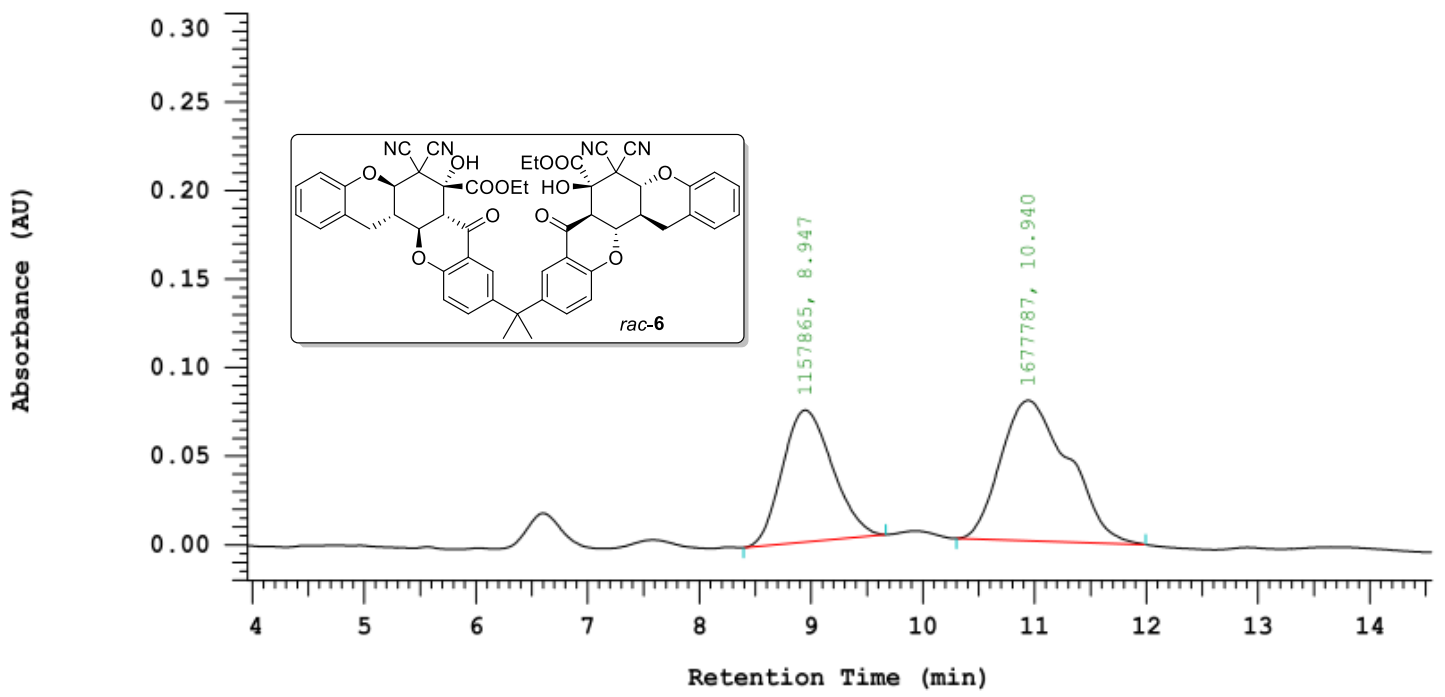

Chrom Type: Fixed WL Chromatogram, $230 \mathrm{~nm}$

Peak Quantitation: AREA

Calculation Method: AREA응

\begin{tabular}{|c|c|c|c|c|}
\hline No. & $\mathrm{RT}$ & Area & Area $\%$ & $\mathrm{BC}$ \\
\hline 1 & 8.947 & 1157865 & 40.832 & BB \\
\hline 2 & 10.940 & 1677787 & 59.168 & $\mathrm{BB}$ \\
\hline & & 2835652 & 100.000 & \\
\hline
\end{tabular}

Chrom Type: Fixed WL Chromatogram, 230 nm

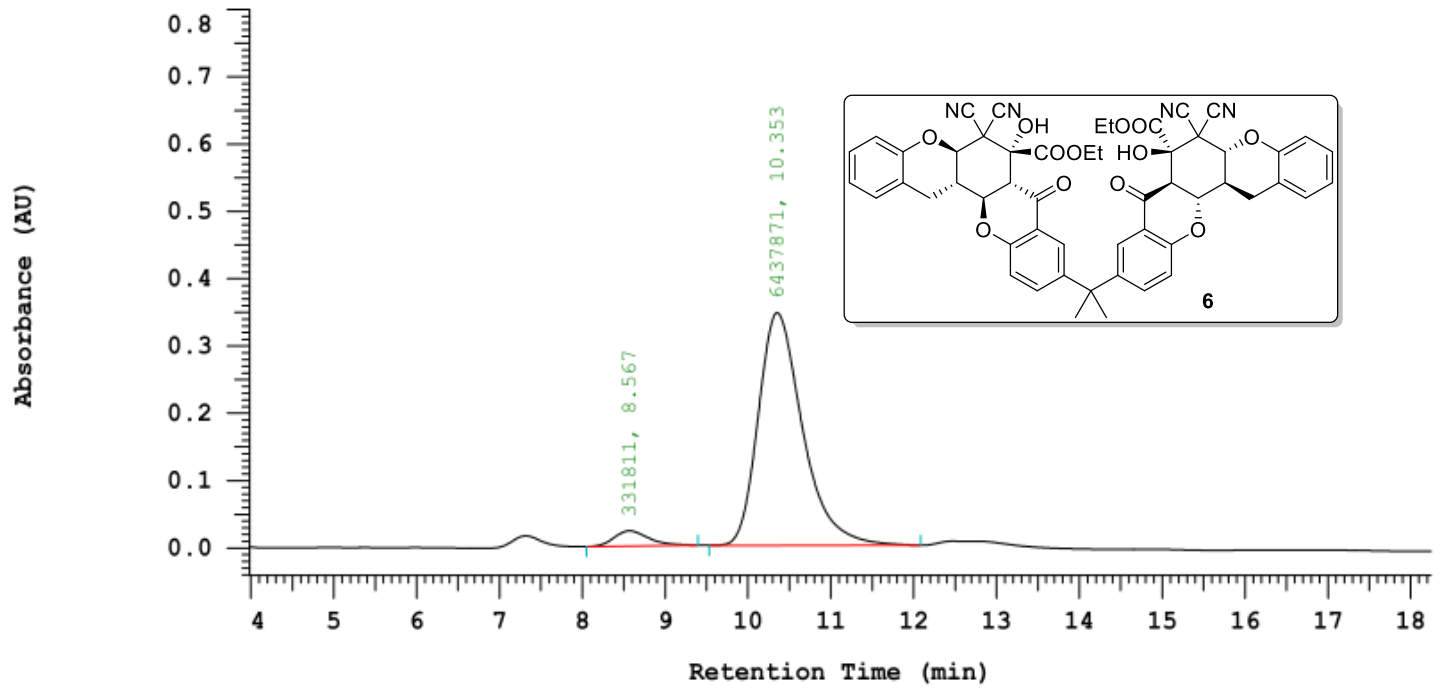

Chrom Type: Fixed WL Chromatogram, 230 nm

Peak Quantitation: AREA

Calculation Method: AREA\%

\begin{tabular}{|c|c|c|c|c|}
\hline No. & RT & Area & Area \% & $\mathrm{BC}$ \\
\hline 1 & 8.567 & 331811 & 4.901 & $\mathrm{BB}$ \\
\hline 2 & 10.353 & 6437871 & 95.099 & $\mathrm{BB}$ \\
\hline & & 6769682 & 100.000 & \\
\hline
\end{tabular}



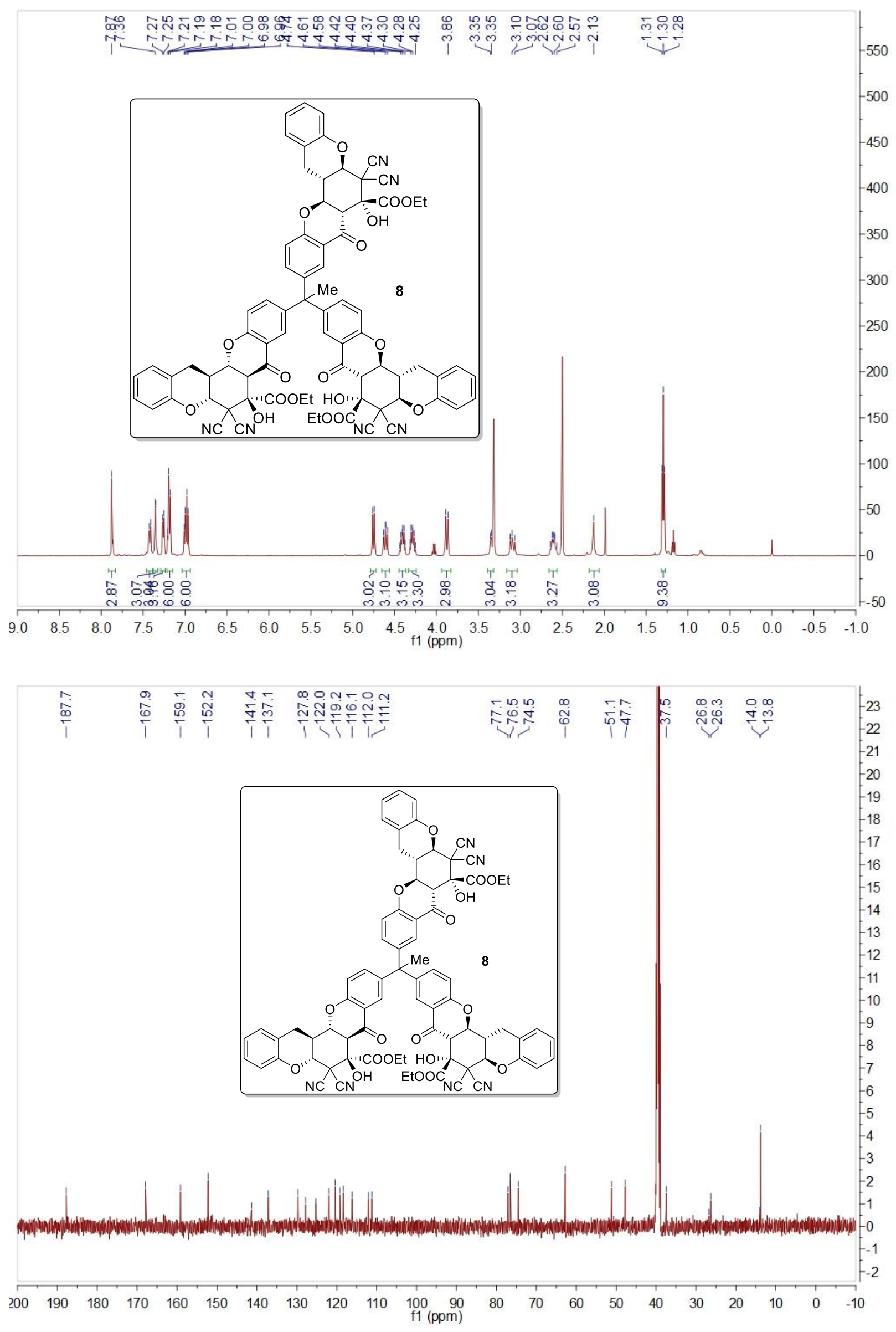
Chrom Type: Fixed WL Chromatogram, 230 nm

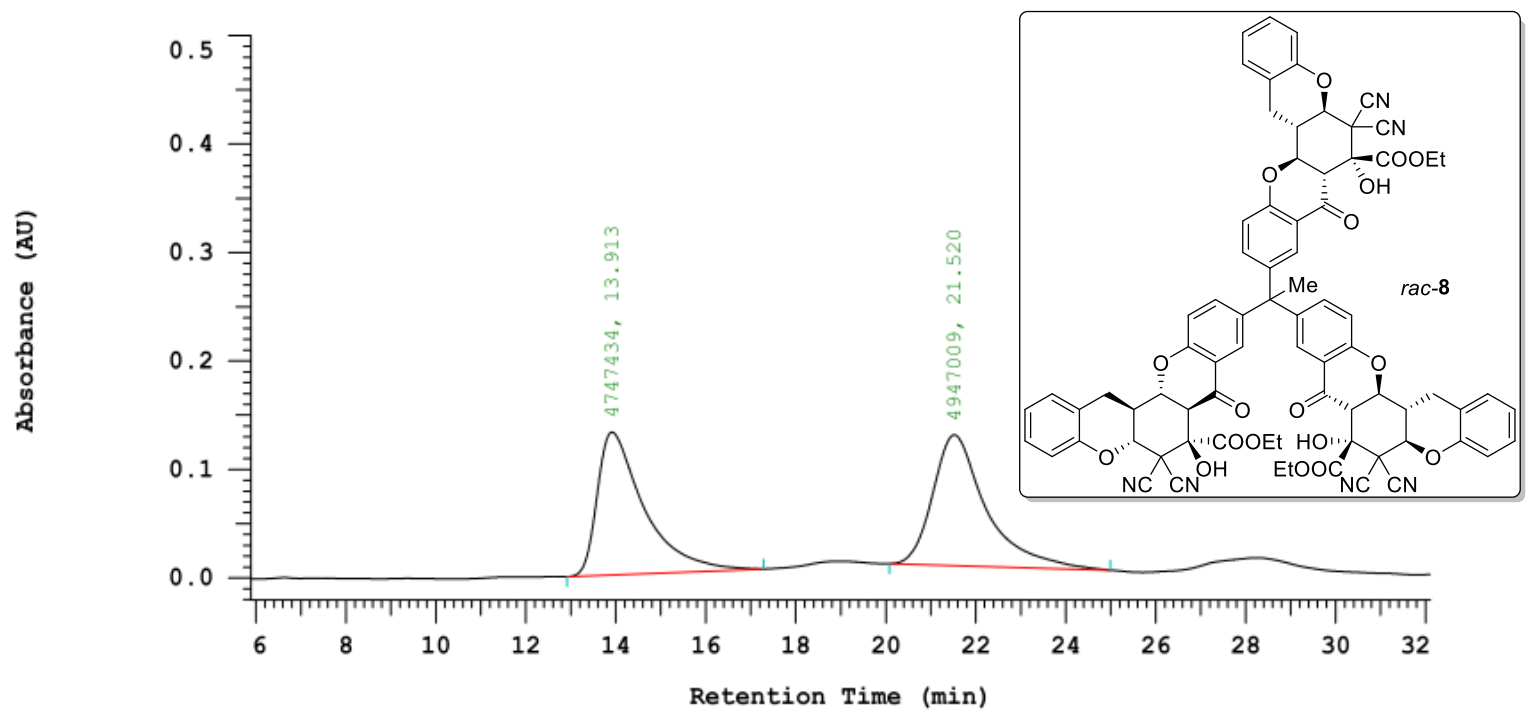

Chrom Type: Fixed WL Chromatogram, $230 \mathrm{~nm}$

Peak Quantitation: AREA

Calculation Method: AREA\%

\begin{tabular}{ccccc} 
No. & RT & Area & Area & BC \\
\hline 1 & 13.913 & 4747434 & 48.971 & BB \\
2 & 21.520 & 4947009 & 51.029 & BB \\
\hline & 9694443 & 100.000 & \\
\hline
\end{tabular}

Chrom Type: Fixed WL Chromatogram, 230 nm

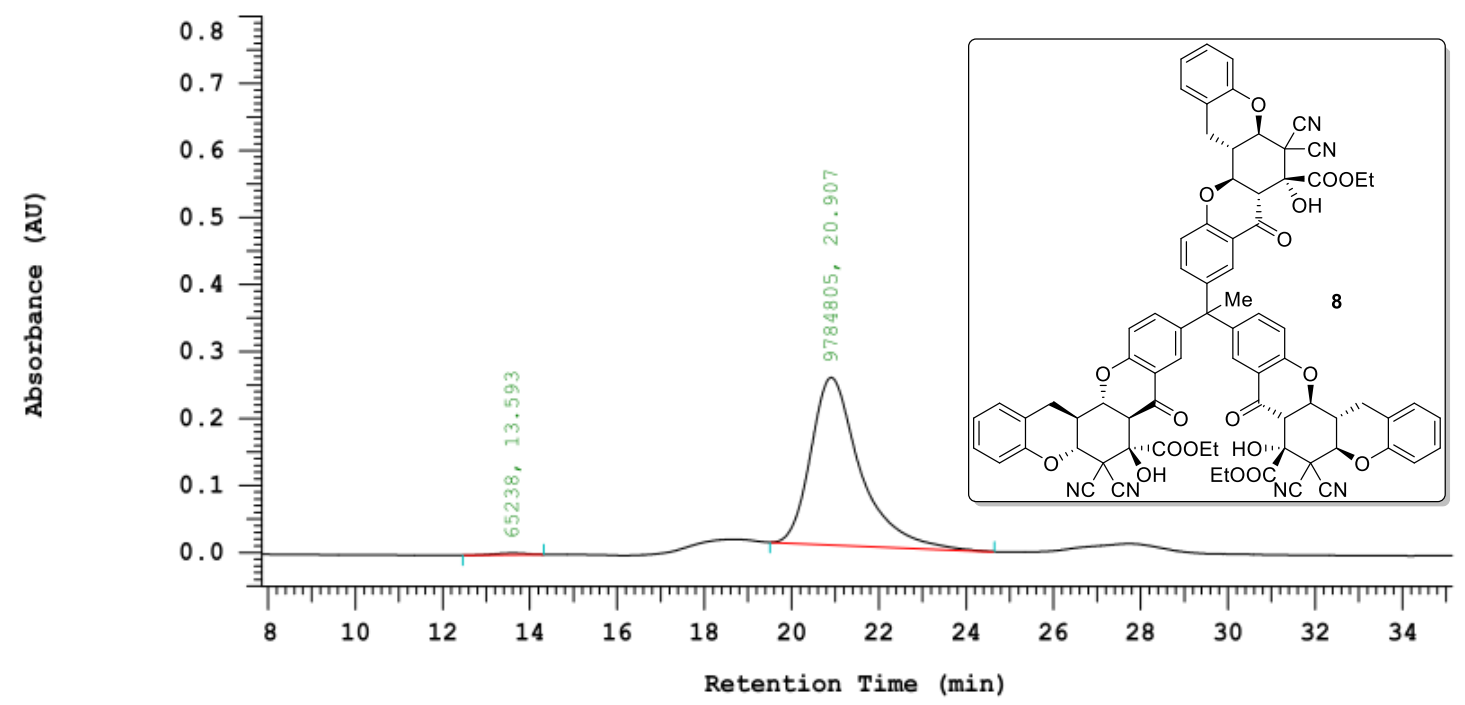

Chrom Type: Fixed WL Chromatogram, $230 \mathrm{~nm}$

Peak Quantitation: AREA

Calculation Method: AREA응

\begin{tabular}{|c|c|c|c|c|}
\hline No. & $\mathrm{RT}$ & Area & Area $\frac{\circ}{\partial}$ & $\mathrm{BC}$ \\
\hline 1 & 13.593 & 65238 & 0.662 & $\mathrm{BB}$ \\
\hline 2 & 20.907 & 9784805 & 99.338 & $\mathrm{BB}$ \\
\hline & & 9850043 & 100.000 & \\
\hline
\end{tabular}

